\title{
DECAY FOR SOLUTIONS OF THE WAVE EQUATION ON KERR EXTERIOR SPACETIMES III: THE FULL SUBEXTREMAL CASE $|a|<M$
}

\author{
MIHALIS DAFERMOS, IGOR RODNIANSKI, AND YAKOV SHLAPENTOKH-ROTHMAN
}

\begin{abstract}
This paper concludes the series begun in [M. Dafermos and I. Rodnianski Decay for solutions of the wave equation on Kerr exterior spacetimes I-II: the cases $|a| \ll M$ or axisymmetry, arXiv:1010.5132], providing the complete proof of definitive boundedness and decay results for the scalar wave equation on Kerr backgrounds in the general subextremal $|a|<M$ case without symmetry assumptions. The essential ideas of the proof (together with explicit constructions of the most difficult multiplier currents) have been announced in our survey [M. Dafermos and I. Rodnianski The black hole stability problem for linear scalar perturbations, in Proceedings of the 12th Marcel Grossmann Meeting on General Relativity, T. Damour et al (ed.), World Scientific, Singapore, 2011, pp. 132189, arXiv:1010.5137]. Our proof appeals also to the quantitative mode-stability proven in [Y. Shlapentokh-Rothman Quantitative Mode Stability for the Wave Equation on the Kerr Spacetime, arXiv:1302.6902, to appear, Ann. Henri Poincaré], together with a streamlined continuity argument in the parameter $a$, appearing here for the first time. While serving as Part III of a series, this paper repeats all necessary notations so that it can be read independently of previous work.
\end{abstract}

\section{Contents}

1. Introduction

1.1. Overview of the main difficulties

1.2. Outline of the paper

1.3. Acknowledgements

2. Review of the setup

2.1. Ambient manifold and coordinate systems

2.2. The Kerr metric and its properties

2.3. Multiplier currents and the general energy identity

3. The main theorems

3.1. Boundedness and integrated local energy decay

3.2. The higher order statement

3.3. Corollaries

3.4. The logic of the proof

4. Preliminaries

4.1. Well posedness, regularity and smooth dependence

4.2. The sign of $a$

4.3. Hardy inequalities

4.4. Generic constants in inequalities and fixed parameters

4.5. The red-shift

4.6. An estimate for large $r$

4.7. A timelike vector field

5. The sufficiently integrable outgoing class and Carter's separation

5.1. The class of sufficiently integrable outgoing functions 
5.2. Review of Carter's separation 23

5.3. Boundary conditions 25

\begin{tabular}{lll} 
5.4. On the almost everywhere regularity of $u_{m \ell}^{(a \omega)}$ & 26 \\
\hline
\end{tabular}

6. Properties of the potential $V \quad 27$

6.1. Admissible frequencies $\quad 27$

\begin{tabular}{lll|} 
6.2. Decomposition of the potential & 28 \\
\hline
\end{tabular}

6.3. The critical points of $V_{0}$ and the structure of trapping $\quad 28$

6.4. Superradiant frequencies are not trapped $\quad 30$

6.5. Trapping for fixed-azimuthal mode solutions $\quad 32$

6.6. Aside: relation with null geodesic flow $\quad 33$

7. The separated current templates $\quad 33$

7.1. The frequency-localised virial currents $\mathbf{J}^{X, w} \quad 34$

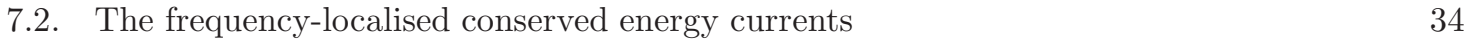

8. The frequency localised multiplier estimates $\quad 35$

\begin{tabular}{|l|l|}
\hline 8.1. The frequency ranges & 36 \\
\hline
\end{tabular}

8.2. Overview $\quad 36$

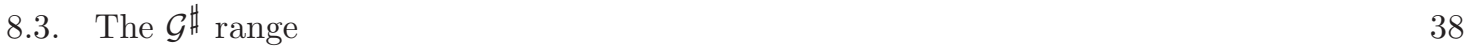

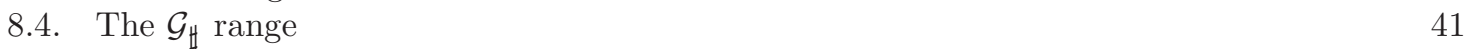

\begin{tabular}{ll|} 
8.5. The $\mathcal{G}_{\hbar}^{\dagger}$ range & 42 \\
\hline
\end{tabular}

\begin{tabular}{lll|} 
8.6. The $\mathcal{G}_{\text {h }}$ range & 44 \\
\hline
\end{tabular}

\begin{tabular}{lll} 
8.7. The $\mathcal{G}_{b}^{\text {b }}$ range & 48 \\
\hline
\end{tabular}

8.8. Putting everything together $\quad 57$

8.9. Trapping parameters $\quad 58$

9. Summing and integrated local energy decay for future-integrable solutions 5

9.1. Future-integrable solutions of the wave equation $\quad 58$

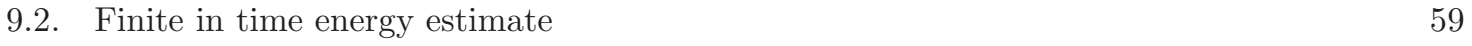

\begin{tabular}{lll|} 
9.3. Adding in the red-shift & 60 \\
\hline
\end{tabular}

\begin{tabular}{lll|} 
9.4. Adding in the large $r$ current & 60 \\
\hline
\end{tabular}

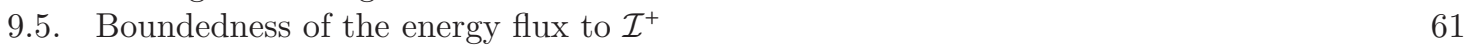

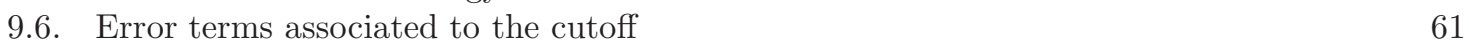

\begin{tabular}{ll|} 
9.7. The non-stationary bounded frequency horizon term & 65 \\
\hline
\end{tabular}

9.8. An inhomogeneous estimate $\quad 65$

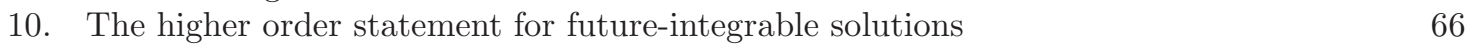

\begin{tabular}{ll|} 
10.1. Elliptic estimates & 67 \\
\hline
\end{tabular}

\begin{tabular}{llr} 
10.2. Proof of Proposition 10.1 & 69 \\
\hline
\end{tabular}

11. The continuity argument $\quad 70$

11.1. The reduction to fixed azimuthal frequency $\quad 70$

\begin{tabular}{lr} 
11.2. Openness & 72 \\
\hline
\end{tabular}

\begin{tabular}{ll|} 
11.3. Closedness & 77 \\
\hline
\end{tabular}

12. The precise integrated local energy decay statement $\quad 78$

13. Energy boundedness $\quad 79$

13.1. Boundedness of $\int_{\Sigma_{\tau} \cap\left[A_{0}+\delta, A_{1}-\delta\right]} \mathbf{J}_{\mu}^{N}[\psi] n_{\Sigma_{\tau}}^{\mu} \quad 79$

13.2. Boundedness of $\int_{\Sigma_{\tau} \cap\left[r_{+}, A_{0}+\delta\right]} \mathbf{J}_{\mu}^{N}[\psi] n_{\Sigma_{\tau}}^{\mu}$ and $\int_{\Sigma_{\tau} \cap\left[A_{1}-\delta, \infty\right)} \mathbf{J}_{\mu}^{N}[\psi] n_{\Sigma_{\tau}}^{\mu}$

13.3. Putting everything together and the higher order statement $\quad 81$

References 


\section{Introduction}

The boundedness and decay properties of solutions to the scalar wave equation

$$
\square_{g_{a, M}} \psi=0
$$

on the exterior regions of Kerr black hole backgrounds $\left(\mathcal{M}, g_{a, M}\right)$ have been the subject of considerable recent activity, in view of the intimate relation of this problem to the stability of these spacetimes themselves in the context of Einstein's theory of general relativity (cf. [20]). Following definitive results [43, 10, 22, 11, 23, 12, 13, 25] in the Schwarzschild case $a=0$, boundedness in the very slowly rotating Kerr case $|a| \ll M$ was first proven in our [26], and subsequently, decay results have been established for $|a| \ll M$ in [27] and in the first parts of this series [30, and independently by Tataru-Tohaneanu [63] and Andersson-Blue [3]. See also [49]. Our [30] also obtained such decay results in the general subextremal case $|a|<M$, under the assumption that $\psi$ is itself axisymmetric. (Let us mention also the previous non-quantitative study [35, 36] of fixed azimuthal modes on Kerr.) The main significance of these restrictive assumptions is that the well-known difficulty of superradiance is controlled by a small parameter (the case $|a| \ll M$ ) or is in fact completely absent (the case of axisymmetric $\psi$ ). The present paper represents the culmination of this programme by dropping these restrictions, extending the above boundedness and decay results to the general subextremal case $|a|<M$ without axisymmetry:

Theorem. 1. General solutions $\psi$ of (11) on the exterior of a Kerr black hole background $\left(\mathcal{M}, g_{a, M}\right)$ in the full subextremal range $|a|<M$, arising from bounded initial energy on a suitable Cauchy surface $\Sigma_{0}$, have bounded energy flux through a global foliation $\Sigma_{\tau}$ of the exterior, bounded energy flux through the event horizon $\mathcal{H}^{+}$and null infinity $\mathcal{I}^{+}$, and satisfy a suitable version of "local integrated energy decay".

2. Similar statements hold for higher order energies involving time-translation invariant derivatives. This implies immediately uniform pointwise bounds on $\psi$ and all translation-invariant derivatives to arbitrary order, up to and including $\mathcal{H}^{+}$, in terms of a sufficiently high order initial energy.

The precise statements of parts 1 and 2 of the above Theorem will be given in Section 3 as Theorems 3.1 and 3.2. Note that these are exact analogues of Theorems 1.2 and 1.3 of [30], dropping the assumption of axisymmetry (alternatively, Theorem 1.1 dropping the assumption $a_{0} \ll M$ ). The main results of the present paper have been previously announced in our survey paper [29], which provided both an outline of the proof and many details of the crucial arguments, including all high frequency multiplier constructions. To complete the outline, one required a quantitative refinement of Whiting's classical mode stability result [65] and a continuity argument in the parameter $a$. The former refinement has very recently been obtained [59] and will be indeed used in our proof. As for the latter, the proof presented here introduces a streamlined continuity argument which as an added benefit in fact reproves the theorems of the first parts of the series [30] in the case $|a| \ll M$. We will only rely on [30] for a detailed discussion of the background, the setup and several of its elementary propositions. All necessary notations and results from [30] are reviewed and quoted explicitly, however, so that the present paper can be read independently. We will also repeat all constructions originally introduced in the survey [29].

In view of our general "black box" decay result [28, a corollary of the above Theorem is

Corollary. Solutions $\psi$ of (1) arising from sufficiently regular and localised initial data (i.e. whose initial suitably higher-order weighted energy is finite) satisfy uniform polynomial decay of the energy flux through a hyperboloidal foliation $\widetilde{\Sigma}_{\tau}$ of the exterior region as well as uniform pointwise polynomial decay bounds.

As in the case of Minkowski space, there is a hierarchy of polynomial decay bounds that can be obtained, both for energy fluxes and pointwise, depending on the quantification of the initial localisation assumed on initial data. The precise statement we shall give (Corollary 3.1] of Section 3) is motivated by applications to quasilinear problems; further refinements fail to be robust from this 
point of view. We remark explicitly that the decay estimates of the above Corollary are indeed sufficient for applications to quasilinear problems with quadratic non-linearities. See [50, 69, 68, 67. We note also that the non-quantitative fixed-azimuthal mode statements of [35, 36] are of course implied a fortiori by the above Corollary. To obtain from our Theorem alternative more refined corollaries for compactly supported smooth initial data, see 62.

As stated, the above Theorem and its Corollary concern the black hole exterior. Note that boundedness and polynomial decay statements in the Kerr exterior propagate easily to any fixed- $r$ hypersurface in the interior following [48] using the red-shift effect and stationarity, for $r$ strictly greater than its value on the Cauchy horizon. This insight goes back to [21. On the other hand, by a result of [56], uniform non-degenerate energy boundedness cannot hold up to the Cauchy horizon in view of the blue-shift. Uniform $L^{\infty}$ bounds on $\psi$ and its tangential derivatives up to and including the Cauchy horizon have been obtained in the thesis of Franzen [37.

Besides the Kerr family, there is an additional class of black hole spacetimes of interest in classical general relativity: the sub-extremal Kerr-Newman metrics. These now form a 3-parameter family of metrics, with parameters $a, M$ and $Q$ (the latter representing charge), which, when coupled with a suitable Maxwell field associated to $Q$, satisfy the Einstein-Maxwell equations. See [39]. (Setting $Q=0$, the Maxwell field vanishes and the family reduces to the Kerr case.) As shown in the thesis of Civin [18, all the miraculous properties of the Kerr family that allow for the results proven in the present paper in fact extend to the Kerr-Newman family in the full sub-extremal parameter range $\sqrt{a^{2}+Q^{2}}<M$, leading to a precise analogue of our Theorem and its Corollary in this more general class.

Though outside of the domain of astrophysically relevant black holes, it is interesting to consider the problem of boundedness and decay for scalar waves on the analogues of the Kerr family when a non-zero cosmological constant $\Lambda$ is added to the Einstein equations. These spacetimes are known as Kerr-de Sitter $(\Lambda>0)$ and Kerr-anti de Sitter $(\Lambda<0)$. See [39. It is in fact the negative case $\Lambda<0$ which presents more surprising new phenomena from the mathematical point of view and has been definitively treated in the work of Holzegel-Smulevici [41, 42. See also [38. The $\Lambda>0$ case is from some points of view easier than $\Lambda=0$, and results in the Schwarzschild-de Sitter $(a=0)$ and very slowly rotating Kerr-de Sitter $(|a| \ll M,|a| \ll \Lambda)$ case followed soon after the analogous results for Schwarzschild and very slowly rotating Kerr had been obtained. See 24, 14, 51, 64, 32, $33,58$. Let us note however, that Kerr-de Sitter is still not understood in its full subextremal range, in particular in view of the absence of an analogue of the mode stability statements [65, 59]. The best results to date have been obtained by Dyatlov [34.1]

Returning to the classical astrophysical domain, let us recall finally that the Kerr metrics $g_{a, M}$ represent black hole spacetimes in the full closed parameter range $|a| \leq M$; the geometry of the extremal case $|a|=M$, however, exhibits several qualitative differences, most conspicuously, the degeneration of the celebrated red-shift effect at the horizon. In view of the recently discovered Aretakis instability [8, 9, 7, 47, 54, the precise analogue of the above Theorem does not in fact hold without qualification in the case of extremality $|a|=M$. In particular, in the extremal case, for generic solutions, certain higher order time-translation-invariant derivatives asymptotically blow up along the event horizon. This generic blow up is completely unrelated to superradiance and holds even for solutions $\psi$ restricted to be axisymmetric. For such axisymmetric $\psi$, restricted decay results of a definitive nature have been obtained by Aretakis [6]. The fundamental remaining problem for scalar waves on black hole backgrounds of interest in classical general relativity is thus the precise

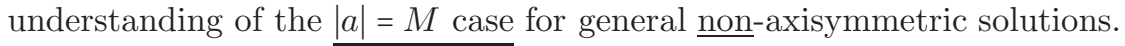

\footnotetext{
${ }^{1}$ Let us remark briefly that besides these extensions to $\Lambda \neq 0$, there are a host of other related problems one can also consider, including higher dimensional black holes [57, 46], other hyperbolic equations like Klein-Gordon (for which it is now proven [60] that there are exponentially growing solutions for all $|a| \neq 0$ ), Maxwell [4, linearised gravity and the nonlinear Einstein vacuum equations themselves (see 40, 31]). We refer the reader to the many additional references in the first part of this series [30, our survey [29] and our lecture notes [27.
} 
1.1. Overview of the main difficulties. We begin with an overview of the difficulties of the problem and the basic elements of the proof. In this discussion, we will assume some familiarity with the Schwarzschild and Kerr families of metrics as well as basic aspects of the analysis of wave equations on Lorentzian manifolds. See our lecture notes [27.

1.1.1. Review of the very slowly rotating case $|a| \ll M$. We have discussed at length in the first parts of this series [30] the various difficulties connected to showing energy boundedness and "integrated local energy decay" for solutions of the wave equation (11) in the very slowly rotating case. We review these briefly.

Already in the Schwarzschild case $a=0$, to show boundedness, one must face the difficulty that at the event horizon $\mathcal{H}^{+}$, the conserved $\partial_{t}$-energy (associated to stationarity of the metric) degenerates. To show integrated local energy decay, one must moreover understand both "low" and "high" frequency obstructions to dispersion, in particular, the high-frequency obstructions connected to the presence of trapped null geodesics.

The horizon difficulties were overcome by our introduction of the red-shift vector field [23], while the difficulties concerning both excluding low frequency obstructions and quantifying the high frequency phenomena connected to trapped geodesics were overcome in one go by appeal to the energy identity of Morawetz-type (cf. [52]) multipliers associated to a vectorfield $f(r) \partial_{r}$, where $f$ is a carefully chosen function vanishing at the so-called photon sphere $r=3 M$, the timelike cylinder to which all trapped null geodesics asymptote. In the Schwarzschild context, such Morawetz estimates were pioneered by [45, 12. The boundedness of the nonnegative space-time integral given by the energy identity associated to this multiplier is precisely the statement of "integrated local energy decay". See also [13, 23]. The degeneracy of any such estimate at trapping is necessary in view of a general result of Sbierski [56] in the spirit of the classical [55. On the other hand, the fact that such a degenerate estimate indeed holds (and the precise nature of the degeneracy) means that the trapping is "good"; at the level of geodesic flow, this corresponds to the fact that dynamics is hyperbolic near the trapped set. This estimate also degenerates at the event horizon when only the initial conserved $\partial_{t}$-energy is included on the right hand side. On the other hand, as shown in [23], again using the red-shift vector field, this degeneracy is removed by replacing the latter with the initial non-degenerate energy.

Turning from Schwarzschild to the very slowly rotating Kerr case $|a| \ll M$, the above difficulties are combined with a new one: superradiance. Now, the vector field $\partial_{t}$ is spacelike in a region outside the horizon known as the ergoregion, hence its energy identity gives no obvious a priori control over the solution. Moreover, it is clear that the high-frequency obstructions to decay cannot be captured from classical physical space vector field multipliers [2]. This can be seen at the level of geodesic flow as the projection of the trapped space to physical space is no longer a codimension- 1 hypersurface.

The problem of superradiance was first overcome in our proof of boundedness [26] mentioned previously, which concerned in fact the more general setting of the wave equation on backgrounds that are small axisymmetric stationary perturbations of Schwarzschild, a class including the very slowly rotating Kerr case $|a| \ll M$. In this class of spacetimes, one can analyse solutions with respect to frequencies $\omega \in \mathbb{R}$ and $m \in \mathbb{Z}$ corresponding to the stationary and axisymmetric Killing fields, and decompose general solutions $\psi$ of (11) into their superradiant and non-superradiant part. For the latter part, one can prove boundedness as in Schwarzschild. For the superradiant part, it turns out that one can explicitly prove both boundedness and integrated local energy decay perturbing a Schwarzschild energy identity yielding both positive boundary and space-time terms that do not degenerate. The non-degeneracy of this estimate encodes the fact that the superradiant part of $\psi$ is not trapped. We shall return to this insight later in our discussion of the general $|a|<M$ case.

Turning to the issue of proving decay for $|a| \ll M$, the problem of capturing the good properties of trapping was overcome using frequency-localised generalisations of the Morawetz multipliers applied in Schwarzschild. There have been three independent approaches [27, 63, 3], which all crucially rely on the additional hidden symmetries of Kerr that are reflected in the existence of a Killing tensor 
and separability properties of both geodesic flow and the wave equation itself. In our approach [27, 30, the frequency localisation uses directly Carter's separation of the wave equation [16, which introduces, in addition to $\omega$ and $m$ above, a real frequency parameter $\lambda_{m \ell}(a \omega)$ parameterised by an additional parameter $\ell \in \mathbb{N}_{0}$ such that, localised to each frequency triple $\left(\omega, m, \lambda_{m \ell}(a \omega)\right)$, the wave equation (11) reduces to the following second order o.d.e.

$$
u^{\prime \prime}+\omega^{2} u=V\left(a \omega, m, \lambda_{m \ell}\right) u
$$

where $V$ is a potential and ' denotes differentiation in a rescaled $r^{*}(r)$ coordinate. The frequencies $\lambda_{m \ell}$ are themselves eigenvalues of an associated elliptic equation whose eigenfunctions (known as oblate spheroidal harmonics) appear in the formula reconstructing $\psi$ from $u$.

Note that in the Schwarzschild $(a=0)$ case, the reduction to (21) corresponds to the classical separation by spherical harmonics, and we have explicitly $\lambda_{m \ell}=\ell(\ell+1)$ (independent of $\omega$ ) and

$$
V_{\text {Schw }}(r)=(r-2 M)\left(\frac{\lambda_{m \ell}}{r^{3}}+\frac{2 M}{r^{4}}\right) .
$$

For all $\lambda_{m \ell}$, the potential $V_{\text {Schw }}$ has a unique simple maximum at an $r$-value $r_{\max }\left(\lambda_{m \ell}\right)$ such that

$$
r_{\max }\left(\lambda_{m \ell}\right) \rightarrow 3 M
$$

as $\ell \rightarrow \infty$. One sees thus the relation of this potential to the trapping phenomenon. Indeed, the radial dependence of null geodesics with fixed energy and angular momentum is governed by a potential which coincides with $V$ above in the high frequency rescaled limit.

In the Kerr case, the eigenvalues $\lambda_{m \ell}(a \omega)$ are no longer explicit expressions, and the form of $V$ is considerably more complicated. It was shown, however, in [27, that for $|a| \ll M$ and for frequencies in the "trapping" regime

$$
1 \ll \omega^{2} \sim \lambda_{m \ell},
$$

the potential $V$ retains its "good" Schwarzschild properties. Specifically, the potential $V$ in (2) can be seen to again have a unique simple maximum in this frequency range, whose $r$-value $r_{\max }$ depends on the frequency parameters

$$
r_{\max }=r_{\max }\left(a \omega, m, \lambda_{m \ell}\right) .
$$

This allows, separately for each $\left(\omega, m, \lambda_{m \ell}(a \omega)\right)$, the construction of an analogue of the current $f(r) \partial_{r}$ vanishing exactly at $r_{\max }$, yielding the desired positivity properties. Unlike the Schwarzschild case, however, there does not exist a unique high frequency limit of $r_{\max }$, consistent with the fact [2] that these currents cannot be replaced by a classical vector field defined in physical space. See however [3]. At the level of geodesic flow, this precisely reflects the fact that trapped null geodesics exist for a full range of $r$-values in a neighbourhood of $r=3 \mathrm{M}$

In the remaining frequency regimes, one can in fact simply carry over the previous physicalspace Schwarzschild constructions (see our argument in [27]) to the more general $|a| \ll M$ case, as, restricted to those ranges, the relevant Schwarzschild estimates do not degenerate and thus their positivity properties are manifestly stable to small perturbation. Alternatively, as in the first parts of this series [30, one can construct new currents taylored specifically to these frequency ranges. The latter approach is more flexible (it has the additional benefit of providing [30] an independent second proof of the Schwarzschild case) and will be more useful for the general subextremal case $|a|<M$.

Let us note that our proof of integrated decay in the first part of this series [30] in fact gives a separate proof of the boundedness statement of [26, when the latter is specialised to Kerr. Here,

\footnotetext{
${ }^{2}$ A posteriori, the good structure of trapping in phase space for $|a| \ll M$ can be understood more conceptually, using the structural stability properties of normal hyperbolicity, provided the latter condition is checked for Schwarzschild; see [66]. Note however that these stability properties depend on strong regularity assumptions on the metric, whereas our original boundedness theorem [26 only requires closeness to Schwarzschild in $C^{1}$. Thus one expects the domain of validity of [26] to be strictly bigger than the class of spacetimes where decay results of the type of our main Theorem hold.
} 
one exploits the fact that when $|a| \ll M$, superradiance is controlled by a small parameter and thus boundary terms of the wrong sign can be absorbed by a small multiple of the red-shift current added to the conserved $\partial_{t}$ energy. One obtains thus boundedness and integrated local energy decay at the same time, without separating the solution into its superradiant and non-superradiant parts. We shall see, however, that for the general case $|a|<M$, the original insight of [26] will again be fundamental.

In discussing our frequency analysis for both the problems of boundedness and integrated local energy decay, we have suppressed an important point: To define frequencies $\omega \in \mathbb{R}$, we are applying the Fourier transform in time. Since solutions are not known a priori to be sufficiently integrable in time, however, one must first apply suitable cutoffs in the future, generating error terms which must then be absorbed. For this, some weak a priori control of these terms is essential-and here we have used in both [26, 30] yet again ${ }^{3}$ the closeness to Schwarzschild. The analogue of this procedure for the general $|a|<M$ case is our appeal to the continuity argument of Section 1.1.5. We defer further discussion of this till then.

1.1.2. Structure of trapping and its disjointness from superradiance. In passing to the general subextremal case $|a|<M$, the first fundamental issues that must be addressed are the "high frequency" ones.

The most obvious question is whether the structure of trapping retains its "good" properties which allow in principle for degenerate integrated decay statements. At the same time, since superradiance is no longer governed by a small parameter, one has to understand what is it which finally quantifies its strength, or, in the context of the proof, how does one guarantee the control of boundary terms in space-time energy identities.

Though geodesic flow and various measures of the strength of superradiance have been thoroughly investigated in physics [15, 17, 61, the properties that turn out to be essential for our argument do not appear to have been considered explicitly in the classical literature. Indeed, it is somewhat of a miracle that the Kerr geometry turns out to be well behaved on both accounts, for the entire subextremal range $|a|<M$, specifically:

(a) The structure of trapping is as in Schwarzschild.

(b) Superradiant frequencies are not trapped.

The embodiment of properties (a) and (b) we shall need were proven already in our survey paper [29] by analysing the potential $V$ in (2). Concerning (a), the "trapping" frequency range (3), the potential $V$ was shown to have a unique simple maximum $V_{\max }$ at a (frequency dependent) $r$ value $r_{\max }$, just as in the slowly rotating case $|a| \ll M$. (This shows a fortiori that the underlying null geodesic flow near trapping is hyperbolic.) Concerning (b), it was shown that for high superradiant frequencies,

$$
1 \ll \lambda+\omega^{2}, \quad \omega\left(\omega-\frac{a m}{2 M\left(M+\sqrt{M^{2}+a^{2}}\right.}\right)<0
$$

the maximum of $V$ is always "quantitatively" above the energy level $\omega^{2}$, in the sense

$$
V_{\max } \geq(1+\epsilon) \omega^{2} \text {. }
$$

(This in turn shows a fortiori that future directed null geodesics whose tangent vector has nonnegative inner product with $\partial_{t}$ are never future trapped; they will always cross the event horizon $\mathcal{H}^{+} 4$ Note that in contrast to the $|a| \ll M$ case, the stronger statement that $\partial_{t}$ is eventually timelike along

\footnotetext{
${ }^{3}$ Let us note that given the boundedness result of [26], then one need not appeal again to closeness to Schwarzschild in the argument for integrated local energy decay; see our original proof in the lecture notes [27. We have used it again in [30] so as for [30] to retrieve independently our previous boundedness result.

${ }^{4}$ In the borderline case when the time frequency $\omega$ vanishes, this is intimately related to the fact that there are no trapped null geodesics orthogonal to $\partial_{t}$. This latter observation turns out to be important in the study of black hole uniqueness (see [1]).
} 
any future trapped null geodesic is not true; i.e. it is not true that all future trapped null geodesics must leave the ergoregion.)

As with the $|a| \ll M$ case, it is not statements about geodesic flow that we appeal to, but rather we use directly the properties of the potential $V$ to construct appropriate energy currents. More specifically, the above properties of the potential are used to construct frequency dependent multiplier currents yielding both positive bulk and positive boundary terms for all high frequency ranges. In the superradiant case, property (5) can be exploited to arrange such that the bulk term is in fact non-degenerate; this can be thought of as the definitive embodiment of (b). Note that these high-frequency multiplier constructions all appeared explicitly in our survey [29. We will repeat these constructions here with very minor modifications. See the outline in Section 1.2 below.

It is interesting to note that property (b) above in fact degenerates in the extremal limit $|a| \rightarrow M$ in the following sense: At the endpoint of the superradiant frequency range (44), one loses the $\epsilon$ in (5). This is an additional (and separate) phenomenon to the degeneration of the red-shift and could have interesting implications for the remaining problem of understanding non-axisymmetric $\psi$ in the extremal case $|a|=M$. See [5] and the discussion in [6] .

1.1.3. Absence of bounded frequency superradiant obstructions. The above still leaves us with the problem of understanding bounded (i.e. $|\omega| \lesssim 1$ ) frequencies.

One must first distinguish the near stationary case $|\omega| \ll 1$. This frequency range is very sensitive to global aspects of the geometry. It turns out that here an explicit multiplier construction is possible which adapts our construction of the first parts of this series [30. (Interestingly, the cases of $|a| \leq \tilde{a}_{0}$ and $|a| \geq \tilde{a}_{0}$ are here handled differently.) These multiplier constructions appear for the first time in the present paper.

Turning now to the remaining bounded frequencies, as explained in our survey [29], whereas in the non-superradiant regime, one can explicitly construct multipliers with both nonnegative bulk and boundary terms, for bounded non-superradiant frequencies, adapting the constructions of 30] from the $|a| \ll M$ case, there does not appear to be a straightforward such construction for the superradiant regime, when neither can superradiance be treated as a small parameter, nor can one exploit (5) together with either $\omega, m$ or $\lambda_{m \ell}$ as a large parameter. One can indeed construct currents with a non-negative bulk term, but these generate a boundary term of the wrong sign which still must be controlled.

As announced already in 29, to control the remaining term one requires a quantitative extension of Whiting's celebrated mode stability [65], which in particular excludes the presence not just of growing modes but also resonances on the real axis. This was achieved in the recent [59]. Appeal to [59] will indeed allow us to control the remaining boundary term. Again, see the outline in Section 1.2 below.

1.1.4. Higher order estimates. To obtain higher-order integrated local energy decay in the slowly rotating case $|a| \ll M$, it was sufficient to commute (11) with $\partial_{t}$ (which is Killing) and also with the red-shift vector field (the latter an argument first applied in [26]), exploiting the fact that the latter, though not Killing, generates positive terms in appropriate energy estimates modulo terms which can be controlled by the $\partial_{t}$-commutation. To show this fact, one uses in turn that control of a second derivative of $\psi$ in a timelike direction allows control of all second derivatives of the solution via elliptic estimates (in view of equation (11)).

For the general case $|a|<M$, one appeals to yet another fundamental fact about Kerr geometry:

(c) The span of the stationary $\partial_{t}$ and axisymmetric $\partial_{\phi}$ Killing fields is timelike outside the horizon for the full range $|a|<M$.

Thus, commuting with $\partial_{t}, \chi \partial_{\phi}$ (where $\chi$ is a cutoff function with compact support in $r$ ) and the red-shift vector field, one can essentially apply the same argument as before.

1.1.5. Continuity argument. We now return to the issue that we have suppressed at the end of Section [1.1.1. namely, the question of how can one justify in the first place a frequency analysis 
based on real frequencies $\omega$ defined via the Fourier transform in time. In the case $|a| \ll M$, closeness to Schwarzschild gave a small parameter that could be exploited here. For the general $|a|<M$ case, however, as explained already in our survey [29], one must exploit a continuity argument in $|a|$.

Note first that to justify the Fourier assumption and thus prove integrated local energy decay, one sees easily that it is sufficient to assume the non-quantitative assumption that the energy through $\Sigma_{\tau}$ of the projection $\psi_{m}$ of $\psi$ to each azimuthal frequency is finite. This is the statement that we show by continuity: For each azimuthal frequency number $m \in \mathbb{Z}$, we define the subset

$$
\mathcal{A}_{m} \subset[0, M)=\left\{|a|: \psi \text { satisfying (11) with } g_{a, M} \Longrightarrow \text { energy of } \psi_{m} \text { remains finite }\right\}
$$

We will show that $\mathcal{A}_{m}$ is a non-empty open and closed subset of $[0, M)$, and thus, $\mathcal{A}_{m}=[0, M)$.

We turn to a brief account of the continuity argument.

The non-emptyness of $\mathcal{A}_{m}$ follows from the general boundedness result for black hole spacetimes without ergoregions proven in 27, specialised to the Schwarzschild case $a=0$.

For openness, one shows that if $\stackrel{\circ}{a} \in \mathcal{A}_{m}$, then $|a-\stackrel{a}{a}|<\epsilon$ satisfies $a \in \mathcal{A}_{m}$ for sufficiently small $\epsilon$. One exploits here $\epsilon$ as a small parameter. The issues associated to openness already appeared in the small $|a| \ll M$ case; see [26] and [30. The fact that we have fixed the azimuthal mode $m$ makes the argument here technically easier to implement. For this, the fundamental insight is that for $m$ fixed

1. trapping occurs outside the ergoregion.

2. using the energy identity for a vector field of the form $\partial_{t}+\alpha(r) \partial_{\phi}$, one can obtain boundedness modulo lower order terms supported only in the ergoregion.

To exploit the above, we first construct from a fixed- $m$ solution $\psi_{m}$ to (11) on $g_{a, M}$ and for each $\tau \geq 0$, a solution $\Psi$ of the inhomogeneous wave equation $\square_{g} \Psi_{m}=F_{m}$ on an interpolating metric $g$ which coincides with $g_{a, M}$ in the region between $\Sigma_{0}$ and $\Sigma_{\tau-\delta_{0}}$ and coincides with $g_{a}, M$ in the region in the future of $\Sigma_{\tau}$ and to which the integrability properties apply (since $\stackrel{\circ}{\in} \in \mathcal{A}_{m}$ ). Applying our estimates and using 1. and 2., we may now absorb (for sufficiently small $\epsilon$ ) the error terms arising from the inhomogeneity to obtain an integrated decay statement for $\psi_{m}$. We note that the fixed- $m$ currents used for 1. and 2. may find additional applications.

Closedness is easy given the estimates shown and the smooth dependence of the Kerr family on the parameter $a$.

1.1.6. Non-degenerate boundedness from integrated local energy decay. The frequency analysis on which our proof of integrated local energy decay is based does not directly "see" the energy flux on fixed time hypersurfaces $\Sigma_{\tau}$, only the energy fluxes on the horizon $\mathcal{H}^{+}$and future null infinity $\mathcal{I}^{+}$. Thus, it remains to show boundedness of the energy (and higher-order energies) through $\Sigma_{\tau}$.

In the slowly rotating case $|a| \ll M$, it is clear that given integrated local energy decay, boundedness of the energy flux through a spacelike foliation easily follows a posterior 5 by revisiting the physical space energy identity of a globally timelike vector field which coincides with $T$ where the latter is timelike, noting that, if $|a|$ is sufficiently small $T$ is timelike near trapping.

The above argument again uses in an essential way the disjointness of the ergoregion and the set-associated to trapping-on which integrated local energy decay estimate degenerates. As we have remarked earlier, these sets intersect when $|a| \sim M$-it is only in phase space where superradiance can be understood as disjoint of trapping.

One approach to boundedness could be to try to exploit again property (b) from Section 1.1.2, It is technically easier to simply exploit the physical space fact (c) of Section 1.1.4. namely that Killing fields $\partial_{t}$ and $\partial_{\phi}$ together span a timelike subspace outside the horizon. Specifically, in a small neighbourhood of any $r$-value there exists a combination of $\partial_{t}$ and $\partial_{\phi}$ which is timelike and Killing. We use our frequency analysis to partition a solution $\psi$ of the wave equation into finitely many pieces $\tilde{\psi}_{i}$, each of which satisfies an analogue of integrated local energy decay degenerating only in a small neighbourhood of some $r_{i}$. Applying the energy estimates corresponding to a suitable $i$-dependent

\footnotetext{
${ }^{5}$ Of course, in our original proof 30], we proved those two statements together as we used the boundedness in our version of the continuity argument. In the new continuity argument presented here, this is not necessary.
} 
combination of $\partial_{t}$ and $\partial_{\phi}$ to each $\tilde{\psi}_{i}$, and summing, one obtains the desired non-degenerate uniform boundedness of the energy flux through $\Sigma_{\tau}$.

1.2. Outline of the paper. We end this introduction with an outline of the structure of the paper. In Section 2] we will review the set-up and various notations from the first parts of the series [30, including the ambient manifold, the form of the Kerr family of metrics and useful vector fields, hypersurfaces and formulas. This will allow us to give precise formulations of the main theorems in Section 3. (The reader may wish to refer to this outline again when reading Section 3.4 which will describe the logical flow of the proofs of the various statements.)

Section 4 contains various preliminaries, including a review of the propositions from [30] capturing the redshift effect, an estimate for large $r$, Hardy inequalities and finally, various statements concerning the span of the Killing fields $\partial_{t}$ and $\partial_{\phi}$.

Our frequency localisation based on Carter's separation will be reviewed in Section 5 The natural setting for this will be the class of sufficiently integrable outgoing functions $\Psi: \mathcal{R} \rightarrow \mathbb{R}$, a useful notion which we shall define in Section [5.1] The resulting coefficients $u$ and their corresponding radial o.d.e. (cf. (21) above) are obtained in Section 5.2 .2 and the "outgoing" boundary conditions in Section 5.1 .2 .

The next three sections, Sections 6, 7, and 8, concern the study of the o.d.e. (2) and the proof of uniform estimates in the frequency parameters $\omega, m$, and $\Lambda$.

In Section 6, we will give salient properties of the potential $V$ of (22) which embody (a) and (b) of Section 1.1.2 Versions of the lemmas of Sections 6.3 and 6.4 together with proofs have in fact already been given in our survey paper 29; we repeat these here for completeness. The lemma of Section 6.5 reflecting the properties of trapping for fixed $m$, is new and will be used in the context of the continuity argument of Section 11 discussed below.

In Section 7 we shall review our notation for fixed frequency current templates, which, upon selection of the free functions, will be used to obtain multiplier estimates for solutions to (2).

Section 8 is the heart of the paper. Here, with the help of well-chosen functions in the current templates of Section 7, we construct suitable currents for all relevant frequency ranges yielding positive bulk terms and thus an estimate for solutions of the radial o.d.e. (21) uniform in frequency parameters. In the trapping regime, the currents degenerate at $r_{\max }$. All these currents have appeared previously in our survey paper [29] with the exception of the near-stationary range of Section 8.7.3 The boundary terms can also be made positive, with the exception of a range of bounded frequencies, which give rise to an extra horizon boundary term on the right hand side of the resulting estimate, which must still be absorbed.

In Section 9, we apply the results of the previous section to the coefficients $u$ arising from the setting of Section 5, summing the resulting frequency localised estimates to obtain control of a nonnegative definite space-time integral. We note Section 9.7, where the extra horizon term (arising from low superradiant frequencies) is bounded by appeal to Proposition 9.7.1] a result of [59. One obtains finally an integrated local decay statement for "future integrable" solutions of the wave equation, and a similar statement for the inhomogeneous equation in Section 9.8

Higher order decay estimates are then provided in Section 10, using the structure described in Section 1.1.4.

In Section 11 we implement our new continuity argument discussed in Section 1.1.5 above, which will allow us to drop the a priori assumption of future integrability, and extend our results to general solutions of the Cauchy problem for (1). The reduction to fixed azimuthal frequency is accomplished in Section 11.1. The most difficult part of the argument is openness, handled in Section 11.2, while closedness is considered in Section 11.3

Section 12 will state the more precise integrated local energy statement which has actually been obtained in the proof.

Finally, in Section 13 we prove the boundedness statements, following our discussion in Section 1.1.6. This will conclude the paper. 
1.3. Acknowledgements. During the period where this research has been carried out, MD was supported by the European Research Council and the Engineering and Physical Sciences Research Council. IR acknowledges support through NSF grant DMS-1001500 and DMS-1065710. YS acknowledges support through NSF grants DMS-0943787 and DMS-1065710. The authors thank S. Aretakis, D. Civin and G. Moschidis for discussions and comments on the manuscript.

\section{REVIEW OF THE SETUP}

In this section, we review the setup and certain notations from the first parts of the series [30, so that the present paper can be read independently. The reader wishing for a more leisurely exposition of this material should refer back to [30; he or she familiar with 30] can skip to Section 3 ,

2.1. Ambient manifold and coordinate systems. The first task is to define an ambient manifoldwith-boundary on which the Kerr family in its subextremal range defines a smooth two-parameter family of metrics. The differential structure of the smooth manifold is defined by what we shall call fixed coordinates, while the Kerr metric itself will be defined with the help of auxilliary coordinates depending on the parameters. We review this here:

2.1.1. Fixed coordinates $\left(y^{*}, t^{*}, \theta^{*}, \phi^{*}\right)$. We define first the manifold-with-boundary

$$
\mathcal{R}=\mathbb{R}^{+} \times \mathbb{R} \times \mathbb{S}^{2} .
$$

Fixed coordinates are just the standard $y^{*} \in \mathbb{R}^{+}, t^{*} \in \mathbb{R}$ and a choice of standard spherical coordinates $\left(\theta^{*}, \phi^{*}\right) \in \mathbb{S}^{2}$. Associated to this ambient differentiable structure are the event horizon $\mathcal{H}^{+} \doteq \partial \mathcal{R}=$ $\left\{y^{*}=0\right\}$, the vector fields $T=\partial_{t^{*}}, \Phi=\partial_{\phi^{*}}$ and the one-parameter group of transormations $\varphi_{\tau}$ generated by $T$.

2.1.2. Kerr-star coordinates $\left(r, t^{*}, \theta^{*}, \phi^{*}\right)$. We define a new coordinate system which depends on parameters $|a|<M$.

For each choice $|a|<M$, we first set $r_{ \pm}=M \pm \sqrt{M^{2}-a^{2}}$ and then define a new coordinate $r$ which is related smoothly to $y^{*}$, depends smoothly on the parameters and such that, for fixed parameters, we have $r=r_{+}(a, M)$ on $\mathcal{H}^{+}$6 Associated to these coordinates is the vector field $Z^{*}$, defined to be the smooth extension of the Kerr-star coordinate vector field $\partial_{r}$ to $\mathcal{R}$.

We will sometimes replace $r$ by a rescaled version, $r^{*}$, defined only in the interior of $\mathcal{R}$, by

$$
\frac{d r^{*}}{d r}=\frac{r^{2}+a^{2}}{\Delta}, \quad r^{*}(3 M)=0,
$$

where $\Delta=\left(r-r_{+}\right)\left(r-r_{-}\right)$. Here we note that $\Delta$ vanishes to first order on $\mathcal{H}^{+}$, and the coordinate range $r>r_{+}$corresponds to the range $r^{*}>-\infty$.

2.1.3. Boyer-Lindquist coordinates $(r, t, \theta, \phi)$. We define a final coordinate system, again depending on a choice of fixed parameters $|a|<M$, by further transforming Kerr star coordinates, by defining

$$
t\left(t^{*}, r\right)=t^{*}-\bar{t}(r), \quad \phi\left(\phi^{*}, r\right)=\phi^{*}-\bar{\phi}(r) \bmod 2 \pi, \quad \theta=\theta^{*}
$$

where $\bar{t}$ is a smooth function (see 30 for details) chosen to satisfy

$$
\begin{gathered}
\bar{t}(r)=r^{*}(r)-r-r^{*}(9 M / 4)+9 M / 4, \quad \text { for } \quad r_{+} \leq r \leq 15 M / 8, \\
\bar{t}(r)=0 \quad \text { for } \quad r \geq 9 M / 4, \\
\frac{d\left(r^{*}-\bar{t}\right)}{d r}>0, \quad 2-\left(1-\frac{2 M r^{2}}{\rho}\right) \frac{d\left(r^{*}-\bar{t}\right)}{d r}>0 .
\end{gathered}
$$

\footnotetext{
${ }^{6}$ The precise relation to fixed coordinates as defined in $\left[30\right.$ is as follows: Let $\mathcal{P}=\left\{\left(x_{1}, x_{2}\right): 0 \leq\left|x_{1}\right|<x_{2}\right\}$ denote the parameter space of all admissible subextremal $(a, M)$. We chose a smooth map $r: \mathcal{P} \times(0, \infty) \rightarrow\left(x_{2}+\sqrt{x_{2}^{2}-x_{1}^{2}}, \infty\right)$ such that $\left.r\right|_{\left\{\left(x_{1}, x_{2}\right)\right\} \times(0, \infty)}$ is a diffeomorphism $(0, \infty) \rightarrow\left(x_{2}+\sqrt{x_{2}^{2}-x_{1}^{2}}, \infty\right)$ which moreover restricts to the identity map restricted to $\left\{\left(x_{1}, x_{2}\right)\right\} \times\left(3 x_{2}, \infty\right)$. Note that with this definition, then for $r \geq 3 M, r\left(y^{*}\right)$ is independent of $a$.
} 
Associated to these coordinates is the vector field $Z$ defined to be (the extension to $\operatorname{int}(\mathcal{R})$ of) the Boyer-Lindquist coordinate vector field $\partial_{r}$ 团

2.2. The Kerr metric and its properties. Given these coordinate systems, we may now define the Kerr metric as a smooth 2-parameter family on $\mathcal{R}$.

2.2.1. Explicit form of the metric. For fixed parameters $|a|<M$, in addition to $\Delta$ above, let us first set $\rho^{2}=r^{2}+a^{2} \cos ^{2} \theta$. The Kerr metric is then defined with respect to Boyer-Lindquist coordinates by

$$
g_{a, M}=-\frac{\Delta}{\rho^{2}}\left(d t-a \sin ^{2} \theta d \phi\right)^{2}+\frac{\rho^{2}}{\Delta} d r^{2}+\rho^{2} d \theta^{2}+\frac{\sin ^{2} \theta}{\rho^{2}}\left(a d t-\left(r^{2}+a^{2}\right) d \phi\right)^{2} .
$$

Though a priori this is only well defined on $\operatorname{int}(\mathcal{R})$, by transforming the above into regular coordinates (see [30]), one sees that the metric (111) extends uniquely to the boundary so that for each $|a|<M$, indeed $\left(\mathcal{R}, g_{a, M}\right)$ defines a smooth Lorentzian manifold-with-boundary, and such that moreover the metric smoothly depends on the parameters $a, M 母$ These metrics are Ricci flat (i.e. they satisfy Einstein's vacuum equations).

2.2.2. Killing fields. We note that the fixed vector fields $T$ and $\Phi$ on $\mathcal{R}$ defined in Section 2.1.2 are Killing for $g_{a, M}$ for all parameter values $|a|<M$.

For each given $|a|<M$, the span of $T$ and $\Phi$ yields a timelike subspace of $T_{p} \mathcal{R}$ for all $p \in \operatorname{int}(\mathcal{R})$ (in particular, $T$ is a timelike vector when $\Phi=0$ ). The event horizon $\mathcal{H}^{+}=\partial \mathcal{R}$ is also a Killing horizon: the Killing field given by the linear combination

$$
K=T+\omega_{+} \Phi,
$$

where $\omega_{+} \doteq \frac{a}{2 M r_{+}}$is the "anuglar velocity" of the event horizon, is null and normal to $\mathcal{H}^{+}$; thus, $\mathcal{H}^{+}$ is in particular a null hypersurface. Note that along $\mathcal{H}^{+}$we have

$$
\nabla_{K} K=\kappa K, \quad \kappa=\frac{r_{+}-r_{-}}{2\left(r_{+}^{2}+a^{2}\right)}>0 .
$$

The quantity $\kappa$ is known as the surface gravity. The positivity (12) is what determines the red-shift property, essential for our estimates (see Section 4.5). We note that $\kappa$ in fact vanishes in the extremal case $|a|=M$; this gives rise to the Aretakis instability [7].

We recall moreover that the vector $K$ restricted to $\mathcal{H}^{+}$coincides with the smooth extension of the coordinate vector field $\partial_{r^{*}}$ of the $\left(r^{*}, t, \theta, \phi\right)$ coordinate system.

2.2.3. The photon sphere and trapping parameters. It is well known that in the Schwarzschild case $a=0$, all future-trapped null geodesics asymptote to the timelike hypersurface $r=3 M$.

In the statement of Theorem 1.1 of [30, we defined $s_{ \pm}\left(a_{0}, M\right)$ such that for all $|a| \leq a_{0}$, then $r_{+}<3 M-s_{-}\left(a_{0}, M\right)$ and all future trapped null geodesics enter the region $3 M-s_{-}\left(a_{0}, M\right)<r<$ $3 M-s_{+}\left(a_{0}, M\right)$. We have shown in Section 10.4 of 29 the existence of such parameters again, for the full subextremal range $|a|<M$. We will repeat this proof in Section 8.9. We note that in the extremal limit $a_{0} \rightarrow M, 3 M-s_{-} \rightarrow r_{+}(M, M)$.

Given the above parameters, let $\eta_{\left[3 M-s^{-}, 3 M+s^{+}\right]}(r)$ denote the indicator function, and let us define, for each $a_{0}<M$, the function

$$
\zeta(r)=(1-3 M / r)^{2}\left(1-\eta_{\left[3 M-s^{-}, 3 M+s^{+}\right]}(r)\right) .
$$

\footnotetext{
${ }^{7}$ Recall that this vector field is significant as it will define the directional derivative that does not degenerate in the integrated decay estimate due to trapping. Note that in Boyer-Lindquist coordinates the fixed vector fields $T$ and $\Phi$ correspond to the coordinate vector fields $\partial_{t}$ and $\partial_{\phi}$.

${ }^{8}$ The latter can be understood in the sense that $g: \mathcal{P} \times \mathcal{R} \rightarrow T^{*} \mathcal{R} \otimes T^{*} \mathcal{R}$

is a smooth map.
} 
This function will encode physical space degeneration of the "integrated local energy decay" estimate of Theorem 3.1. The presence of the $(1-3 M / r)^{2}$ factor ensures uniformity of the estimate as $a_{0} \rightarrow 0$ so as to retrieve our original Schwarzschild result [23].

Finally, since it is derivatives with respect to the vector field $Z$ which do not degenerate at trapping, but it is the vector field $Z^{*}$ which extends to the horizon, it will be convenient to define a hybrid vector field that has both good properties. For this let us define, for each $|a|<M$, a cutoff funtion $\chi(r)$ such that $\chi=1$ for $r \geq 3 M-s^{-}$and $\chi=0$ for $r \leq\left(r_{+}+3 M-s^{-}\right) / 2$, We define then a new vector field $\tilde{Z}^{*}=\chi Z+(1-\chi) Z^{*}$. This will be the vector field which appears in the statement of Theorem 3.1 .

2.2.4. The ergoregion. The region $\mathcal{S} \subset \mathcal{R}$ where $T$ is spacelike is known as the ergoregion; more explicitly, it is exactly the subset of $\mathcal{R}$ defined by

$$
\mathcal{S}=\left\{\Delta-a^{2} \sin ^{2} \theta<0\right\} .
$$

The boundary $\partial \mathcal{S}$ is called the ergosphere.

2.2.5. The $\Sigma_{\tau}$ hypersurfaces, and the regions $\mathcal{R}_{(0, \tau)}, \mathcal{H}_{(0, \tau)}^{+}$. We have arranged the definition of Kerrstar coordinates in Section 2.1.2 so that the hypersurfaces $t^{*}=c$ are spacelike (see the conditions (10)) with respect to the metric $g_{a, M}$, for all values of parameters $|a|<M$.

In the region $r \leq 15 \mathrm{M} / 8$, we have in fact

We will define

$$
g\left(\nabla t^{*}, \nabla t^{*}\right)=-1-\frac{2 M r}{\rho^{2}} .
$$

$\Sigma_{\tau}=\left\{t^{*}=\tau\right\}$,

$$
\mathcal{R}_{(0, \tau)}=\cup_{0 \leq \tau^{*} \leq \tau} \Sigma_{\tau^{*}}
$$

and

$$
\mathcal{R}_{0}=\cup \mathcal{R}_{(0, \tau)} .
$$

Note that $\Sigma_{0}$ is a past Cauchy hypersurface for the regions $\mathcal{R}_{(0, \tau)}, \mathcal{R}_{0}$. Let us also define

$$
\mathcal{H}_{(0, \tau)}^{+}=\mathcal{R}_{(0, \tau)} \cap \mathcal{H}^{+}, \quad \mathcal{H}_{0}^{+}=\mathcal{R}_{0} \cap \mathcal{H}^{+} .
$$

2.2.6. Angular derivatives and the volume form. For future reference, let us introduce here the notation $\not, \not \nabla$ to denote the induced metric and covariant derivative from $g_{a, M}$ (11) on the $\mathbb{S}^{2}$ factors of $\mathcal{R}$ in the product (6).

We record finally from [30] some useful properties of the volume form $d V$ of the metric $g_{a, M}$ : With respect to Boyer-Lindquist coordinates, we have

$$
d V=v(r, \theta) d t d r d V_{\not j} \quad \text { with } v \sim 1
$$

whereas using the alternative $r^{*}$ coordinate,

$$
d V=v\left(r^{*}, \theta\right) d t d r^{*} d V_{\not j} \quad \text { with } v \sim \Delta / r^{2} .
$$

With respect to Kerr-star coordinates, we have

$$
d V=v\left(r, \theta^{*}\right) d t^{*} d r d V_{\not g} \quad \text { with } v \sim 1 .
$$

Let $\gamma$ denote the standard unit metric on the sphere in $(\theta, \phi)$ coordinates. We have that $\not \sim r^{2} \gamma$, and thus we may replace $d V_{\not}$ in the above using

$$
d V_{\phi}=v(r, \theta) r^{2} \sin \theta d \theta d \phi \quad \text { with } v \sim 1 .
$$

Finally, we note that

$$
d V \sim d \tau d V_{\Sigma_{\tau}} .
$$


For $a_{0}<M$ and $|a| \leq a_{0}$, note that the implicit constants in the above are uniformly bounded, depending only on $a_{0}$ and $M$.

2.3. Multiplier currents and the general energy identity. We shall repeat our standard notation for vector field multiplier current identities associated to "multiplier" vector fields $V$ which will be applied to $\psi$ as well as to $\Xi \psi$ for various commutation vector fields $\Xi$. See [30] for more details and 19] for a systematic discussion. See 44 for an early application of non-trivial energy currents to the problem of decay for the wave equation on Minkowski space.

2.3.1. Currents. Given a general Lorentzian manifold $(\mathcal{M}, g)$, let $\Psi$ be a sufficiently regular function. We define

$$
\mathbf{T}_{\mu \nu}[\Psi] \doteq \partial_{\mu} \Psi \partial_{\nu} \Psi-\frac{1}{2} g_{\mu \nu} g^{\alpha \beta} \partial_{\alpha} \Psi \partial_{\beta} \Psi
$$

Given a sufficiently regular vector field $V_{\mu}$ and function $w$ on $\mathcal{M}$, we will define the currents

$$
\begin{gathered}
\mathbf{J}_{\mu}^{V}[\Psi]=\mathbf{T}_{\mu \nu}[\Psi] V^{\nu}, \quad \mathbf{J}_{\mu}^{V, w}[\Psi]=\mathbf{J}_{\mu}^{V}[\Psi]+\frac{1}{8} w \partial_{\mu}\left(\Psi^{2}\right)-\frac{1}{8}\left(\partial_{\mu} w\right) \Psi^{2}, \\
\mathbf{K}^{V}[\Psi]=\mathbf{T}_{\mu \nu}[\Psi] \nabla^{\mu} V^{\nu}, \quad \mathbf{K}^{V, w}[\Psi]=\mathbf{K}^{V}[\Psi]-\frac{1}{8} \square_{g} w\left(\Psi^{2}\right)+\frac{1}{4} w \nabla^{\alpha} \Psi \nabla_{\alpha} \Psi, \\
\mathcal{E}^{V}[\Psi]=-\left(\square_{g} \Psi\right) V^{\nu} \Psi_{, v}, \quad \mathcal{E}^{V, w}[\Psi]=\mathcal{E}^{V}(\Psi)-\frac{1}{4} w \Psi \square_{g} \Psi .
\end{gathered}
$$

Remark 2.3.1. Note that even if one is only interested in the study of solutions $\psi$ to the homogeneous (1), inhomogeneous terms will arise from applying cutoffs to $\psi$ and also from applying commutation vector fields (like vector field $Y$ from Section 4.5 below) which do not commute with $\square_{g}$.

2.3.2. The divergence identity. The divergence identity between two homologous spacelike hypersurfaces $S^{-}, S^{+}$, bounding a region $\mathcal{B}$, with $S^{+}$in the future of $S^{-}$, yields

$$
\int_{S^{+}} \mathbf{J}_{\mu}^{V}[\Psi] n_{S^{+}}^{\mu}+\int_{\mathcal{B}}\left(\mathbf{K}^{V}[\Psi]+\mathcal{E}^{V}[\Psi]\right)=\int_{S^{-}} \mathbf{J}_{\mu}^{V}[\Psi] n_{S^{-}}^{\mu},
$$

where $n_{\Sigma_{i}}$ denotes the future directed timelike unit normal. The induced volume forms are to be understood. A similar identity holds for the $\mathbf{J}_{\mu}^{V, w}$ currents, etc.

We shall typically apply (16) for the Kerr metric $g_{a, M}$ in the case where $S^{-}=\Sigma_{0}$ and $S^{+}=$ $\Sigma_{\tau} \cup \mathcal{H}_{(0, \tau)}^{+}$and $\Psi$ is compactly supported in $\mathcal{R}_{(0, \tau)}$ to obtain

$$
\int_{\Sigma_{\tau}} \mathbf{J}_{\mu}^{V}[\Psi] n_{\Sigma_{\tau}}^{\mu}+\int_{\mathcal{H}_{(0, \tau)}^{+}} \mathbf{J}_{\mu}^{V}[\Psi] n_{\mathcal{H}^{+}}^{\mu}+\int_{\mathcal{R}_{(0, \tau)}}\left(\mathbf{K}^{V}[\Psi]+\mathcal{E}^{V}[\Psi]\right)=\int_{\Sigma_{0}} \mathbf{J}_{\mu}^{V}[\Psi] n_{\Sigma_{0}}^{\mu}
$$

Let us note that the compactness of the support justifies the absence of an additional boundary term even though $S^{ \pm}$are not homologous. Since $\mathcal{H}^{+}$is null, its induced normal form is coupled to the choice of $n_{\mathcal{H}^{+}}^{\mu}$. In writing the above, we shall assume such a choice has been made such that the formula indeed holds.

2.3.3. Superradiance in Kerr. As already mentioned in the introduction, the presence of the ergoregion $\mathcal{S}$ is one of the fundamental difficulties associated with the passage from Schwarzschild to a rotating Kerr spacetime. One particular consequence is that for $a \neq 0$, the conserved $\mathbf{J}_{\mu}^{T}[\psi]$ energy flux for a solution to (1) may be negative on the horizon $\mathcal{H}^{+}$. Hence, applying (17), the energy on $\Sigma_{\tau}$ can be larger than the energy on $\Sigma_{0}$; this phenomenon is known as superradiance 0

An explicit computation in (17) shows the $\mathbf{J}_{\mu}^{T}[\psi]$ energy flux along $\mathcal{H}^{+}(0, \infty)$ is given by

$$
\int_{\mathcal{H}^{+}(0, \infty)} \operatorname{Re}\left(T \psi \overline{\left(T \psi+\omega_{+} \Phi \psi\right)}\right)
$$

\footnotetext{
${ }^{9}$ In this context, it is in fact more appropriate to refer to the energy flux to null infinity $\mathcal{I}^{+}$.
} 
where $\omega_{+}$was defined in Section 2.2.2. In particular, if one formally considers a (complex-valued) solution of the form

$$
\psi\left(t^{*}, r, \theta, \phi^{*}\right)=e^{-i \omega t^{*}} e^{i m \phi^{*}} \psi_{0}(r, \theta),
$$

then the sign of the $\mathbf{J}_{\mu}^{T}[\psi]$ flux on the horizon is determined by the sign of

$$
\omega\left(\omega-\omega_{+} m\right) .
$$

Thus, we say that the parameters $\omega$ and $m$ are superradiant if

$$
\omega\left(\omega-\omega_{+} m\right)<0 .
$$

Observe that in the case $a \geq 0$, the condition (18) is equivalent to

$$
m \omega \in\left(0, \frac{a m^{2}}{2 M r_{+}}\right]
$$

We will return to a discussion of the significance of this frequency range in Section 6.4

\section{THE MAIN THEOREMS}

With the notations of Section 2 we may now give precise statements of the results.

3.1. Boundedness and integrated local energy decay. Recall the notations of Section 2, in particular the hypersurfaces $\Sigma_{\tau}$, the region $\mathcal{R}_{0}$, the vector fields $T, \tilde{Z}^{*}$ and the degeneration function $\zeta$ defined in (13). Let $n_{\Sigma_{\tau}}^{\mu}, n_{\mathcal{H}^{+}}^{\mu}$ denote the corresponding normals. The vector field $N$ below can be taker 10 to be $n_{\Sigma_{\tau}}$, thought of now as a smooth vectorfield on $\mathcal{R}$.

The main theorem of the present paper is

Theorem 3.1. Fix $M>0,0 \leq a_{0}<M$ and $\delta>0$. There exists a constant $C=C\left(a_{0}, M, \delta\right)$ such that for all $|a| \leq a_{0}$, and all sufficiently regular solutions $\psi$ of the wave equation $\square_{g_{a, M}} \psi=0$ on $\mathcal{R}_{0}$, the following estimates hold:

$$
\begin{gathered}
\int_{\mathcal{R}_{0}}\left(r^{-1} \zeta|\not \psi|^{2}+r^{-1-\delta} \zeta(T \psi)^{2}+r^{-1-\delta}\left(\tilde{Z}^{*} \psi\right)^{2}+r^{-3-\delta}\left(\psi-\psi_{\infty}\right)^{2}\right) \leq C \int_{\Sigma_{0}} \mathbf{J}_{\mu}^{N}[\psi] n_{\Sigma_{0}}^{\mu}, \\
\int_{\mathcal{H}_{0}^{+}}\left(\mathbf{J}_{\mu}^{N}[\psi] n_{\mathcal{H}^{+}}^{\mu}+\left(\psi-\psi_{\infty}\right)^{2}\right) \leq C \int_{\Sigma_{0}} \mathbf{J}_{\mu}^{N}[\psi] n_{\Sigma_{0}}^{\mu}, \\
\int_{\mathcal{I}^{+}} \mathbf{J}_{\mu}^{T}[\psi] n_{\mathcal{I}^{+}}^{\mu} \leq C \int_{\Sigma_{0}} \mathbf{J}_{\mu}^{N}[\psi] n_{\Sigma_{0}}^{\mu}, \\
\int_{\Sigma_{\tau}} \mathbf{J}_{\mu}^{N}[\psi] n_{\Sigma_{\tau}}^{\mu} \leq C \int_{\Sigma_{0}} \mathbf{J}_{\mu}^{N}[\psi] n_{\Sigma_{0}}^{\mu}, \quad \forall \tau \geq 0,
\end{gathered}
$$

where $4 \pi \psi_{\infty}^{2}=\lim _{r^{\prime} \rightarrow \infty} \int_{\Sigma_{0} \cap\left\{r=r^{\prime}\right\}} r^{-2} \psi^{2}$.

Estimate (20) is an integrated local energy decay statement degenerating at trapping. The full statement obtained in the proof is more precise but cannot be expressed in physical space; see Proposition 12.1 of Section 12 .

Estimate (21) is the boundedness of the energy flux through the event horizon $\mathcal{H}_{0}^{+}$(as measured by a local observer), while estimate (22) is the boundedness of the energy flux to null infinity $\mathcal{I}^{+}$. (The latter will be explained in Section 9.5) These two estimates are obtained concurrently with (20).

\footnotetext{
${ }^{10}$ We can alternatively take $N$ to be the vector field of Proposition 4.5.1 this is the vector field we shall use in the proof. For the statement of Theorem 3.1 the only important feature of $N$ is that it is $\phi_{\tau}$-invariant, strictly timelike and asymptotic to $T$ for large $r$. Whereas we could have used everywhere $n_{\Sigma_{\tau}}$ in the statement, we prefer to keep the distinct roles of $n_{\Sigma_{\tau}}$ and $N$ as this will be important when we replace $\Sigma_{\tau}$ with hyperboloidal hypersurfaces $\widetilde{\Sigma}_{\tau}$ in Section 3.3
} 
Estimate (23) is the statement of uniform energy boundedness through the foliation $\Sigma_{\tau}$. We note that the proof of this statement, which is obtained a posteriori, requires the more precise version of (20) given in Proposition 12.1] Note that

$$
\int_{\Sigma_{\tau}} \mathbf{J}_{\mu}^{N}[\psi] n_{\Sigma_{\tau}}^{\mu} \sim\|\psi\|_{H^{1}\left(\Sigma_{\tau}\right)}^{2}+\left\|n_{\Sigma_{\tau}} \psi\right\|_{L^{2}\left(\Sigma_{\tau}\right)}^{2} \sim \int_{\theta, \phi^{*}} \int_{r_{+}}^{\infty}\left(\left|\partial_{t^{*}} \psi\right|^{2}+\left|\partial_{r} \psi\right|^{2}+|\not \psi \psi|_{\not g}^{2}\right) d r d V_{\not g}
$$

with respect to coordinates $\left(t^{*}, r, \theta, \phi^{*}\right)$, where here $f(\psi) \sim g(\psi)$ means there exist constants $c$ and $C$ not depending on $\psi$ such that $c g(\psi) \leq f(\psi) \leq C g(\psi)$. Thus, (23) gives uniform geometric $\dot{H}^{1}$ bounds on the solution.

The reader familiar with Penrose-diagrammatic notation may find the following useful

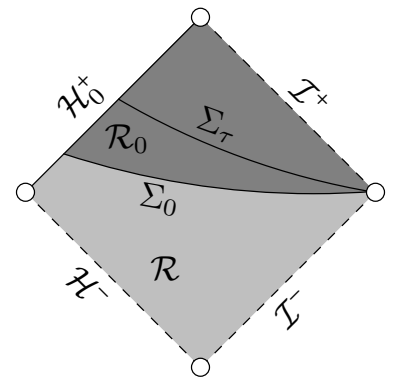

3.2. The higher order statement. For various applications, it is essential to have a higher-order analogue of the above. This is given by

Theorem 3.2. Let $M, a_{0}, a$ be as in Theorem 3.1. Then, for all $\delta>0$ and all integers $j \geq 1$, there exists a constant $C=C\left(a_{0}, M, \delta, j\right)$ such that the following inequalities hold for all sufficiently regular solutions $\psi$ to the wave equation $\square_{g_{a, M}} \psi=0$ on $\mathcal{R}_{0}$

$$
\begin{aligned}
& \int_{\mathcal{R}_{0}} r^{-1-\delta} \zeta \sum_{1 \leq i_{1}+i_{2}+i_{3} \leq j}\left|\not^{i_{1}} T^{i_{2}}\left(\tilde{Z}^{*}\right)^{i_{3}} \psi\right|^{2} \\
&+r^{-1-\delta} \sum_{1 \leq i_{1}+i_{2}+i_{3} \leq j-1}\left(\left|\not^{i_{1}} T^{i_{2}}\left(\tilde{Z}^{*}\right)^{i_{3}+1} \psi\right|^{2}+\left|\not^{i_{1}} T^{i_{2}}\left(Z^{*}\right)^{i_{3}} \psi\right|^{2}\right) \\
& \leq C \int_{\Sigma_{0}} \sum_{0 \leq i \leq j-1} \mathbf{J}_{\mu}^{N}\left[N^{i} \psi\right] n_{\Sigma_{0}}^{\mu}, \\
& \int_{\mathcal{H}_{0}^{+}} \sum_{0 \leq i \leq j-1} \mathbf{J}_{\mu}^{N}\left[N^{i} \psi\right] n_{\mathcal{H}^{+}}^{\mu} \leq C \int_{\Sigma_{0}} \sum_{0 \leq i \leq j-1} \mathbf{J}_{\mu}^{N}\left[N^{i} \psi\right] n_{\Sigma_{0}}^{\mu}, \\
& \int_{\mathcal{I}^{+}} \sum_{0 \leq i \leq j-1} \mathbf{J}_{\mu}^{T}\left[N^{i} \psi\right] n_{\mathcal{I}^{+}}^{\mu} \leq C \int_{\Sigma_{0}} \sum_{0 \leq i \leq j-1} \mathbf{J}_{\mu}^{N}\left[N^{i} \psi\right] n_{\Sigma_{0}}^{\mu}, \\
& \int_{\Sigma_{\tau}} \sum_{0 \leq i \leq j-1} \mathbf{J}_{\mu}^{N}\left[N^{i} \psi\right] n_{\Sigma_{\tau}}^{\mu} \leq C \int_{\Sigma_{0}} \sum_{0 \leq i \leq j-1} \mathbf{J}_{\mu}^{N}\left[N^{i} \psi\right] n_{\Sigma_{0}}^{\mu}, \quad \forall \tau \geq 0 .
\end{aligned}
$$

Let us note that by an elliptic estimate, we have

$$
\int_{\Sigma_{\tau}} \sum_{0 \leq i \leq j-1} \mathbf{J}_{\mu}^{N}\left[N^{i} \psi\right] n_{\Sigma_{\tau}}^{\mu} \sim \sum_{1 \leq i \leq j}\|\psi\|_{H^{i}\left(\Sigma_{\tau}\right)}^{2}+\left\|n_{\Sigma_{\tau}} \psi\right\|_{H^{i-1}\left(\Sigma_{\tau}\right)}^{2} .
$$

Thus, as with (23) before, we may reexpress statement (28) of the above theorem as the statement of the uniform boundedness of geometric Sobolev norms. Note that uniform pointwise bounds on $|\psi|$ and its derivatives to arbitrary order $\left|\not^{i_{1}} T^{i_{2}}\left(\tilde{Z}^{*}\right)^{i_{3}} \psi\right|$ in $\mathcal{R}_{0}$ follow as an immediate consequence of the above Theorems in view of the Sobolev inequality applied on each $\Sigma_{\tau}$.

The above theorems also imply pointwise decay statements and decay for the energy flux through suitable hypersurfaces. We turn to this now. 
3.3. Corollaries. Let us note first that by a reduction proven as Proposition 4.6 .1 of [30, Theorems 3.1 and 3.2 hold where $\Sigma_{0}$ is replaced by an arbitrary "admissible" hypersurface $\widetilde{\Sigma}_{0}$ (see Section 4.4 of [30] for this notion), $\Sigma_{\tau}$ is replaced by $\widetilde{\Sigma}_{\tau} \doteq \varphi_{\tau}\left(\widetilde{\Sigma}_{0}\right), n_{\Sigma_{\tau}}$ is replaced by $n_{\widetilde{\Sigma}_{\tau}}, \mathcal{R}_{0}$, $\mathcal{H}_{0}^{+}$are redefined as $D^{+}\left(\widetilde{\Sigma}_{0}\right), D^{+}\left(\widetilde{\Sigma}_{0}\right) \cap \mathcal{H}^{+}$, respectively, and $N$ is kept as is. This notion includes both asymptotically flat hypersurfaces terminating at spatial infinity (a special case of admissible hypersurfaces of the first kind) and asymptotically hyperboloidal hypersurfaces terminating at null infinity (a special case of admissible hypersurface of the second kind). The latter case is depicted below

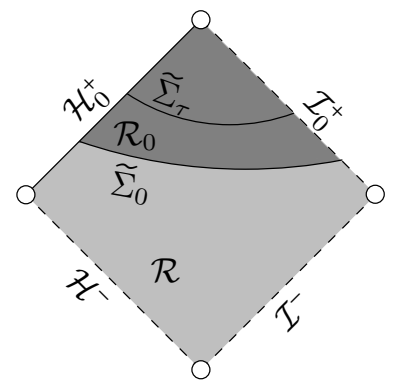

Note, however, that in the latter case, (29) (with the above substitutions) will never hold. It is for this reason that we prefer to state Theorems 3.1 and 3.2 in the form given.

As a consequence of this more general statement, the above theorems allow us to apply our "black box" result of [28] (see [57] and [53] for detailed treatments). We obtain

Corollary 3.1. Let $a_{0}, M, a, \delta$ be as in Theorems 3.1 3.2. and let $R>r_{+}$. Let $\widetilde{\Sigma}_{0}$ be an asymptotically hyperboloidal hypersurface terminating at null infinity, and denote $\widetilde{\Sigma}_{\tau}=\varphi_{\tau}\left(\widetilde{\Sigma}_{0}\right)$. Then for sufficiently regular solutions of the wave equation, we have the following estimates for the energy flux

$$
\begin{gathered}
\int_{\widetilde{\Sigma}_{\tau}} \mathbf{J}_{\mu}^{N}[\psi] n_{\widetilde{\Sigma}_{\tau}}^{\mu} \leq C\left(a_{0}, M\right) E \tau^{-2} \\
\int_{\widetilde{\Sigma}_{\tau} \cap\{r \leq R\}} \mathbf{J}_{\mu}^{N}[N \psi] n_{\widetilde{\Sigma}_{\tau}}^{\mu} \leq C\left(a_{0}, M, \delta, R\right) E \tau^{-4+2 \delta}
\end{gathered}
$$

and the following pointwise estimates

$$
\begin{gathered}
\sup _{\widetilde{\Sigma}_{\tau}} r\left|\psi-\psi_{\infty}\right| \leq C\left(a_{0}, M\right) \sqrt{E} \tau^{-1 / 2}, \\
\sup _{\widetilde{\Sigma}_{\tau} \cap\{r \leq R\}}\left|\psi-\psi_{\infty}\right| \leq C\left(a_{0}, M, \delta, R\right) \sqrt{E} \tau^{-3 / 2+\delta}, \\
\sup _{\widetilde{\Sigma}_{\tau} \cap\{r \leq R\}}\left|n_{\widetilde{\Sigma}} \psi\right|+\left|\nabla_{\widetilde{\Sigma}} \psi\right| \leq C\left(a_{0}, M, \delta, R\right) E \tau^{-2+\delta},
\end{gathered}
$$

where in each inequality, $E$ denotes an appropriate higher order weighted energy on $\widetilde{\Sigma}_{0}$ (or alternatively on an asymptotically flat $\Sigma_{0}$ in the past of $\widetilde{\Sigma}_{0}$ ).

From the point of view of nonlinear applications, the main significance of the powers on the right hand side of (30) and (31) is that they are integrable in time.

3.4. The logic of the proof. Now that we have given precise formulations of the main theorems, we will give a brief account of the logic of the proof, highlighting where each statement is proven.

The reader may wish to refer back to the outline of Section 1.2, Recall that Section 4 concerns various preliminary propositions, including a reduction (in Section 4.1) to considering $\psi$ arising from smooth compactly supported data on $\Sigma_{0}$, whereas Section 5 defines a class of functions for which Carter's separation to the radial o.d.e. (44) and appropriate boundary conditions can be justified $a$ priori. Sections 6 7 and 8 on the other hand, are logically independent of the rest of the paper; they 
are concerned with the study of classical solutions $u$ to the o.d.e. (44) assumed to satisfy appropriate boundary conditions. The culmination is Theorem 8.1 which establishes estimates on $u$ independent of the frequency parameters in the potential.

The logic of the proof of Theorem 3.1 can be properly thought to commence in Section 9 We define a class of solutions to (1) which we call "future-integrable" and which allows us to apply Carter's separation of Section 5 to a suitably defined function, with the help of a cutoff. We then apply Theorem 8.1 to the resulting $u$. Summing via Plancherel, and using in addition the preliminary propositions of Section 4 and the refined mode stability of [59], we establish in Proposition 9.1.1 the integrated energy decay statement (20), the horizon energy flux bound (21) and the null infinity flux bound (22) for this class of future-integrable solutions to (1).

In Proposition 10.1 we will upgrade these to the higher order statements (25), (27) and (28) of Theorem 3.2, again for the class of future-integrable solutions.

Next, in Proposition 11.1 we will use a continuity argument to show that all solutions to (1) arising from smooth compactly supported data (according to the reduction of Section 4.1) are futureintegrable. We thus unconditionally obtain the statements (20), (21), (22), (25), (26) and (27).

Finally, in Proposition 13.1 we unconditionally establish the statements (23) and (28). This will complete the proof of Theorems 3.1 and 3.2 .

\section{Preliminaries}

4.1. Well posedness, regularity and smooth dependence. Let us note that the wave equation (11) is well posed in $\mathcal{R}_{0}$ with initial data $\left(\psi, \psi^{\prime}\right)$ defined on $\Sigma_{0}$ in $H_{\text {loc }}^{j}\left(\Sigma_{0}\right) \times H_{\text {loc }}^{j-1}\left(\Sigma_{0}\right)$ (cf. Proposition 4.5.1 of [30]). Moreover, if the initial data are smooth and of compact support on $\Sigma_{0}$, then $\psi$ will be smooth, and of compact support on all $\Sigma_{\tau}$ for $\tau \geq 0$.

In the proof of our theorems, by standard density arguments (applied to $\psi-\psi_{\infty}$ ), we may thus assume that $\psi$ indeed arises from such data and thus is smooth and of compact support for fixed $\Sigma_{\tau}$ for all $\tau \geq 0$.

Lastly, we observe that the solution $\psi$ to (11) depends smoothly on $a$, e.g.

Lemma 4.1.1. Let $\left|a_{\infty}\right|<M,\left\{a_{k}\right\}_{k=1}^{\infty}$ satisfy $a_{k} \rightarrow a_{\infty}, \square_{g_{a_{k}, M}} \psi_{k}=0, \psi_{k}\left|\Sigma_{0}=\psi_{\infty}\right| \Sigma_{0}$ and $n_{\Sigma_{0}} \psi_{k} \mid \Sigma_{0}=$ $\left.n_{\Sigma_{0}} \psi_{\infty}\right|_{\Sigma_{0}}$. Then, for every $j \geq 1$ and $\tau \geq 0$,

$$
\left.\lim _{k \rightarrow \infty} \int_{\Sigma_{\tau}} \sum_{1 \leq i_{1}+i_{2}+i_{3} \leq j}|\not|^{i_{1}} T^{i_{2}}\left(\tilde{Z}^{*}\right)^{i_{3}} \psi_{k}\right|^{2}=\int_{\Sigma_{\tau}} \sum_{1 \leq i_{1}+i_{2}+i_{3} \leq j}\left|\not \hbar^{i_{1}} T^{i_{2}}\left(\tilde{Z}^{*}\right)^{i_{3}} \psi_{\infty}\right|^{2} .
$$

(We shall appeal to the above lemma at the end of Section 11.3 in the context of the closedness part of our continuity argument.)

4.2. The sign of $a$. For given $a, M$, given a solution $\psi$ of $\square_{g_{M, a}} \psi=0$, then, defining $\tilde{\psi}\left(y^{*}, t^{*}, \theta^{*}, \phi^{*}\right)=$ $\psi\left(y^{*}, t^{*}, \theta^{*}, 2 \pi-\phi^{*}\right)$, we have that $\tilde{\psi}$ satisfies $\square_{g_{M,-a}} \tilde{\psi}=0$. Moreover, the estimates of Theorems 3.1 and 3.2 for $\tilde{\psi}$ with quantities defined respect to the metric $g_{M,-a}$ are equivalent to the analogous estimates for $\psi$ with respect to the metric $g_{M, a}$. Thus, it suffices to prove our Theorems for $a \geq 0$. This reduction is of no conceptual significance, but it slightly simplifies the notation for discussing the superradiant frequency range, which then can be given by (19). For notational convenience we will indeed use the reduction to $a \geq 0$ in Sections 68 the context of describing the properties of the potential $V$ in various frequency regimes and defining the frequency dependent multiplier currents. The reader can assume that $a \geq 0$ globally in this paper, but it is strictly speaking only necessary for those statements which refer explicitly to frequency-dependent functions in the separation.

4.3. Hardy inequalities. As in the previous parts [30] of this series, at various points we shall refer to Hardy inequalities. In view of our comments concerning the volume form (see Section 2.2.6), the reader can easily derive these from the one-dimensional inequalities

$$
\int_{0}^{2} x^{-1}|\log x|^{-2} f^{2}(x) \leq C \int_{0}^{2}\left(\frac{d f}{d x}\right)^{2}(x) d x+C \int_{1}^{2} f^{2}(x) d x,
$$




$$
\int_{1}^{\infty} f^{2}(x) \leq C \int_{1}^{\infty} x^{2}\left(\frac{d f}{d x}\right)^{2}(x) d x
$$

where the latter holds for functions $f$ of compact support.

4.4. Generic constants in inequalities and fixed parameters. Let us recall our conventions from [30] regarding constants depending on the Kerr geometry.

As in the statement of Theorem 3.1 all propositions in this paper providing estimates will explicitly refer to two fixed parameters $a_{0}<M$ delineating the range of Kerr parameters allowed. In the context of inequalities, we shall denote by $B$ potentially large positive constants, whereas we shall denote by $b$ potentially small positive constants, both depending only on $M$ and $a_{0}$. This dependence is always to be understood. We record the resulting algebra of constants:

$$
b+b=b, B+B=B, B \cdot B=B, B^{-1}=b, \ldots
$$

We note that these constants will often blow up $B \rightarrow \infty, b^{-1} \rightarrow \infty$ in the extremal limit $a_{0} \rightarrow M$.

Our constructions will depend on various additional parameters, for instance, the parameters $\omega_{\text {high }}, E$, etc., which are free in the statements of Propositions 8.3.1. etc., but are chosen by the end of the proof of Theorem 8.1] in whose statement they appear as fixed parameters.

When a parameter is required to be "sufficiently large" or "sufficiently small" without further clarification, this always means that there exists a constant depending on $a_{0}$ and $M$ such that the parameter can be taken to be an arbitrary value bigger than that constant. If a parameter is required to be "sufficiently large" given another parameter, this means that there again exists such a constant depending on $a_{0}$ and $M$ and the other parameter.

Until a parameter has been fixed, e.g. the parameter $\omega_{\text {high }}$, we shall use the notation $B\left(\omega_{\text {high }}\right)$, etc., in the context of inequalities, to denote constants depending on $\omega_{\text {high }}$ in addition to $M$ and $a_{0}$. For a parameter, say $c$ which is an explicit function of other parameter(s), say $\omega_{\text {high }}$, together with $M$ and $a_{0}$, we will write $c\left(\omega_{\text {high }}\right)$. Again, the dependence on $M$ and $a_{0}$ is always to be understood.

The final choices of all initially free parameters used in the present paper will be made to depend only on $M$ and $a_{0}$. Once such choices are made, $B\left(\omega_{\text {high }}\right)$ is replaced by $B$, following our conventions.

4.5. The red-shift. Understanding the red-shift is an essential part of the dynamics. Definitive constructions have been given in Section 7 of [27. These depend only on the positivity of the surface gravity $\kappa$, recalled in Section 2.2 .2

4.5.1. The vectorfield $N$. Let us recall briefly from [30] the construction of a vector field $N$ capturing the red-shift effect.

Proposition 4.5.1. Let $|a| \leq a_{0}<M, g_{a, M}$ be the Kerr metric and $\mathcal{R}$, etc., be as before. There exist positive constants $b$ and $B$, parameters $r_{1}(a, M)>r_{\text {red }}(a, M)>r_{+}$and $a \varphi_{\tau}$-invariant timelike vector field $N=N(a, M)$ on $\mathcal{R}$, normalised so that $N-K$ is future oriented, traverse to $\mathcal{H}^{+}$, and null with $g(N, K)=-2$, such that

(1) $\mathbf{K}^{N}[\Psi] \geq b \mathbf{J}_{\mu}^{N}[\Psi] N^{\mu}$ for $r \leq r_{\text {red }}$

(2) $-\mathbf{K}^{N}[\Psi] \leq B \mathbf{J}_{\mu}^{N}[\Psi] N^{\mu}$, for $r \geq r_{\text {red }}$

(3) $T=N$ for $r \geq r_{1}$,

where the currents are defined with respect to $g_{a, M}$.

Note the implicit $a_{0}$ and $M$ dependence of constants $b$ and $B$ as described in Section 4.4 above. This proposition would fail in the case $a_{0}=M$. See [6, 56].

4.5.2. The red-shift estimate. The above leads immediately to the following estimate (see [30])

Proposition 4.5.2. Let $g=g_{a, M}$ for $|a| \leq a_{0}<M$, and let $r_{\text {red }}$ be as in the above Proposition. Then the following is true. For all $r_{+} \leq \tilde{r} \leq r_{\text {red }}$ and $\tilde{\delta}>0$, there exists a positive constant $B(\tilde{r}, \tilde{\delta})$, such 
that for all functions $\Psi$ on $\mathcal{R}_{0}$, then

$$
\begin{gathered}
\int_{\mathcal{R}_{(0, \tau)} \cap\{r \leq \tilde{r}\}}\left(\mathbf{J}_{\mu}^{N}[\Psi] N^{\mu}+\left|\log \left(\left|r-r_{+}\right|\right)^{-2} \| r-r_{+}\right|^{-1} \Psi^{2}\right)+\int_{\mathcal{H}_{(0, \tau)}^{+}} \mathbf{J}_{\mu}^{N}[\Psi] n_{\mathcal{H}^{+}}^{\mu}+\int_{\Sigma_{\tau} \cap\{r \leq \tilde{r}\}} \mathbf{J}_{\mu}^{N}[\Psi] n^{\mu} \\
\leq B(\tilde{r}, \tilde{\delta}) \int_{\Sigma_{0}} \mathbf{J}_{\mu}^{N}[\Psi] n^{\mu}+B(\tilde{r}, \tilde{\delta}) \int_{\mathcal{R}_{(0, \tau)} \cap\{\tilde{r} \leq r \leq \tilde{r}+\tilde{\delta}\}}\left(\mathbf{J}_{\mu}^{N}[\Psi] N^{\mu}+\Psi^{2}\right)-\mathcal{E}^{N}[\Psi] .
\end{gathered}
$$

Again, recall that the additional dependence of $B$ on $M$ and $a_{0}$ is now implicit according to our conventions. Note that the proof of this estimate uses the Hardy inequality (32), so as to include the useful zeroth order term on the left hand side. We note that the same estimate holds with the above zeroth order terms removed from both the right and the left hand sides.

4.5.3. Red-shift commutation and the vector field $Y$. We specialise Theorem 7.2 of 27 to the Kerr case.

Proposition 4.5.3. Let $g=g_{a, M}$, let $K$ be the vector field of Section 2.2.2, let $Y=N-K$, and let $E_{1}, E_{2}$ be $\varphi_{\tau}$-invariant vector fields such that $\left\{K, Y, E_{1}, E_{2}\right\}$ form a local null frame on $\mathcal{H}^{+}$. Then for all $k \geq 0$ and multi-indices $\mathbf{m}=\left(m_{1}, m_{2}, m_{3}, m_{4}\right)$,

$$
\square_{g}\left(Y^{k} \Psi\right)=\kappa_{k} Y^{k+1} \Psi+\sum_{|\mathbf{m}| \leq k+1, m_{4} \leq k} c_{\mathbf{m}} E_{1}^{m_{1}} E_{2}^{m_{2}} L^{m_{3}} Y^{m_{4}} \Psi+Y^{k}\left(\square_{g} \Psi\right)
$$

where $\kappa_{k}>0$ and the $c_{\mathbf{m}}$ are smooth $\varphi_{\tau}$-invariant functions.

The above proposition, which is another manifestation of the red-shift effect, effectively allows us not only to apply a transversal vector field to the horizon as a multiplier, but also as a commutation vector field. This is fundamental for retrieving higher order statements as in Theorem 3.2 .

4.6. An estimate for large $r$. We will also need the following estimate.

Proposition 4.6.1. Fix $M>0$ and $a_{0}<M$. For each $\delta>0$, there exist positive values $2 M<\tilde{R}<$ $R_{\text {large }}$, and positive constants $B(\delta)$ such that if $|a| \leq a_{0}, \psi$ denotes a solution of (11) and $\psi_{\infty}=0$, then for all $\tau \geq 0$

$$
\begin{aligned}
& \int_{\mathcal{R}_{(0, \tau)} \cap\left\{r \geq R_{\text {large }}\right\}} r^{-1}\left(r^{-\delta}\left|\partial_{r} \psi\right|^{2}+r^{-\delta}\left|\partial_{t} \psi\right|^{2}+|\not \psi \psi|_{\not g}^{2}+r^{-2-\delta} \psi^{2}\right) \\
& \leq B(\delta) \int_{\Sigma_{0}} \mathbf{J}_{\mu}^{N}[\psi] n_{\Sigma_{0}}^{\mu}+B(\delta) \int_{\Sigma_{\tau}} \mathbf{J}_{\mu}^{N}[\psi] n_{\Sigma_{\tau}}^{\mu}+B(\delta) \int_{\mathcal{R}_{(0, \tau)} \cap\{r \geq \tilde{R}\}} r^{1+\delta}|F|^{2} \\
& +B(\delta) \int_{\mathcal{R}_{(0, \tau)} \cap\left\{\tilde{R} \leq r \leq R_{\text {large }}\right\}}\left(\left|\partial_{r} \psi\right|^{2}+\left|\partial_{t} \psi\right|^{2}+\left.|\not| \psi\right|_{\not g} ^{2}+\psi^{2}\right) .
\end{aligned}
$$

The homogeneous case is treated in [30, and the inhomogeneous case follows by the same argument.

4.7. A timelike vector field. We have noted in Section 2.2.2 that the span of $T$ and $\Phi$ is a null subspace on the horizon $\mathcal{H}^{+}$and a timelike subspace on $\mathcal{R} \backslash \mathcal{H}^{+}$. These statements are in particular implied by the following Lemma, which will be important later.

Lemma 4.7.1. The vector field

$$
T+\frac{2 M a r}{\left(r^{2}+a^{2}\right)^{2}} \Phi
$$

is timelike in $\mathcal{R} \backslash \mathcal{H}^{+}$and null on $\mathcal{H}^{+}$.

Proof. On the horizon $\mathcal{H}^{+}$, the vector field reduces to

$$
T+\frac{a}{2 M r_{+}} \Phi
$$

which can immediately be seen to be its standard null generator. 
Off the horizon, computing $g\left(T+\frac{2 M a r}{\left(r^{2}+a^{2}\right)^{2}} \Phi, T+\frac{2 M a r}{\left(r^{2}+a^{2}\right)^{2}} \Phi\right)$ in Boyer-Lindquist coordinates reduces the assertion to checking that

$$
-\Delta+\sin ^{2} \theta\left(a^{2}-\frac{4 M^{2} r^{2} a^{2}}{\left(r^{2}+a^{2}\right)^{2}}-\frac{4 M^{2} r^{2} a^{4} \sin ^{2} \theta \Delta}{\left(r^{2}+a^{2}\right)^{4}}\right)<0 .
$$

It suffices to consider the case where the quantity in parentheses is positive. In this case, it is sufficient to check that

$$
-\Delta+a^{2}-\frac{4 M^{2} r^{2} a^{2}}{\left(r^{2}+a^{2}\right)^{2}}<0
$$

Observe that

$$
\left(r^{2}+a^{2}\right)^{2}=(\Delta+2 M r)^{2}=\Delta^{2}+4 M r \Delta+4 M^{2} r^{2} .
$$

Multiplying through by $\left(r^{2}+a^{2}\right)^{2}$ then reduces (34) to

$$
-\Delta^{3}-4 M r \Delta^{2}-4 M^{2} r^{2} \Delta+a^{2} \Delta^{2}+4 M r a^{2} \Delta<0 \Leftrightarrow-\Delta^{2}-\left(4 M r-a^{2}\right) \Delta-4 M r\left(M r-a^{2}\right)<0 .
$$

Now it suffices to observe the inequalities $r>M>a$.

We also note the following, which actually is a trivial consequence of positivity of surface gravity (12).

Lemma 4.7.2. There exists $\epsilon_{0}>0$ such that the vector field

$$
T+\frac{a}{2 M r_{+}} \Phi
$$

is timelike for $r \in\left(r_{+}, r_{+}+\epsilon_{0}\right)$.

Proof. A computation gives

$$
g\left(T+\frac{a}{2 M r_{+}} \Phi, T+\frac{a}{2 M r_{+}} \Phi\right)=\rho^{-2}\left(-\Delta+\sin ^{2} \theta\left(a^{2}-\frac{2 a^{2} r}{r_{+}}+\frac{a^{2}\left(r^{2}+a^{2}\right)^{2}}{4 M^{2} r_{+}^{2}}-\frac{a^{4} \sin ^{2} \theta \Delta}{4 M^{2} r_{+}^{2}}\right)\right)
$$

Consider the function

$$
F(r):=-\Delta+a^{2}-\frac{2 a^{2} r}{r_{+}}+\frac{a^{2}\left(r^{2}+a^{2}\right)^{2}}{4 M^{2} r_{+}^{2}} .
$$

The lemma follows noting that $F\left(r_{+}\right)=0$ and

$$
\begin{aligned}
\frac{d F}{d r}\left(r_{+}\right) & =-\left(r_{+}-r_{-}\right)-\frac{2 a^{2}}{r_{+}}+\frac{2 a^{2}}{M} \\
& =-2 \sqrt{M^{2}-a^{2}}-\frac{2 a^{2}}{M+\sqrt{M^{2}-a^{2}}}+\frac{2 a^{2}}{M} \\
& =\frac{1}{M\left(M+\sqrt{M^{2}-a^{2}}\right)}\left(-2 M\left(M^{2}-a^{2}\right)-2 \sqrt{M^{2}-a^{2}}\left(M^{2}-a^{2}\right)\right) \\
& <0 .
\end{aligned}
$$

\section{The SUfFiciently integrable outgoing ClAss And Carter's SEPARAtion}

In this section we will define a suitable class of functions $\Psi$ for which one can apply Carter's separation, and for which moreover, the resulting radial ordinary differential equation for $u$ will satisfy appropriate "outgoing" boundary conditions. We shall define this class in Section 5.1 below, and then review the separation in Section 5.2 . 
5.1. The class of sufficiently integrable outgoing functions. We define here a class of functions $\Psi$ for which frequency analysis is well defined. To give some motivation for the class, we give a brief preview of how separation will be applied.

As described in the introduction, the frequency analysis necessary for our proof of integrated local energy decay requires taking a Fourier transform in $t$. However, a priori, solutions $\psi$ to the wave equation (10) could even grow exponentially in time. We shall at first instance, however, restrict to smooth solutions of the wave equation (arising from compactly supported data) which are assumed to be $L^{2}$ in time in the future of $\Sigma_{0} 11$ We shall first appeal to our estimates with $\Psi=\xi \psi$ where $\psi$ is a solution which is known to be future integrable, and $\xi(\tau)$ is a suitable cutoff so that $\xi=1$ for $\tau \geq 1$ and $\xi=0$ for $\tau \leq 0$. See Proposition 9.1.1. Note that $\Psi$ satisfies an inhomogeneous equation

$$
\square_{g_{a, M}} \Psi=F
$$

with compactly supported $F$, and $\Psi=0$ to the past of $\Sigma_{0}$.

In the context of the openness argument, we shall apply estimates to $\Psi=\xi \psi$ with two different choices of $\psi$ and the cutoff $\xi(\tau)$. First, we will take $\psi$ to solve the wave equation (11), and the cutoff $\xi(\tau)$ will be taken to vanish for $\tau \leq 0$ and $\tau \geq \tau_{0}$. Next, we will take $\psi$ to be be a solution of $\square_{g} \psi=0$ where $g$ is an "interpolating metric" between $g_{a, M}$ and a $g_{a, M}$, with interpolation region between hypersurfaces $\Sigma_{\tau-\delta_{0}}$ and $\Sigma_{\tau}$, and $\xi(\tau)$ will be take to equal 1 for $\tau \geq 1$ and equal 0 for $\tau \leq 0$. This will make $\Psi$ an $L^{2}$ in time solution of the inhomogeneous (36), where again $F$ is compactly supported in spacetime and $\Psi=0$ in the past of $\Sigma_{0}$. See Proposition 11.1.2 and Section 11.2.2.

In the closedness argument, we shall be able to work with solutions $\psi$ which are a priori square integrable in time. Thus, we shall apply our estimates to $\Psi=\xi \psi$ where $\xi(\tau)$ is an appropriate cutoff such that $\xi=1$ for $\tau \geq 1$ and $\xi=0$ for $\tau \leq 0$. Then, $\Psi$ will satisfy an inhomogeneous equation (36) with a compactly supported right hand side, and $\Psi=0$ to the past of $\Sigma_{0}$.

Finally, in the context of the boundedness argument, $\psi$ has already been proven to be $L^{2}$ in time (both to the future, and, after suitable extension, to the past), and the argument is applied to $\Psi=\tilde{\chi} \psi$ where $\tilde{\chi}(r)$ is a cutoff in $r$ away from the horizon and null infinity. See Section 13

In all these cases, we note that we apply frequency analysis to $\Psi$ which satisfies (a) $\Psi(r)$ is square integrable in $t$ for each $r \in\left[r_{+}, \infty\right)$. and (b) $\Psi$ is supported away from the past event horizon $\mathcal{H}^{-}$and "past null infinity" $\mathcal{I}^{-}$(cf. the Penrose diagram of Section 3.1), with $\square_{g_{a, M}} \Psi$ vanishing for sufficiently large $r$. It is these properties that motivate the following definitions.

5.1.1. Sufficiently integrable. We first introduce the most basic integrability assumptions that will allow us to apply the separation and make sense of the radial o.d.e. (21) discussed already in Section 1.1.1.

Definition 5.1.1. Let $a_{0}<M,|a| \leq a_{0}$ and let $g=g_{a, M}$. We say that a smooth function $\Psi: \mathcal{R} \rightarrow \mathbb{R}$ is "sufficiently integrable" if for every $j \geq 1$ and $A>r_{+}$, we have

$$
\begin{gathered}
\left.\sup _{r \in\left[r_{+}, A\right]} \int_{-\infty}^{\infty} \int_{\mathbb{S}^{2}} \sum_{0 \leq i_{1}+i_{2}+i_{3} \leq j}|\not|^{i_{1}} T^{i_{2}}\left(Z^{*}\right)^{i_{3}} \Psi\right|^{2} \sin \theta d t d \theta d \phi<\infty, \\
\left.\sup _{r \in\left[r_{+}, A\right]} \int_{-\infty}^{\infty} \int_{\mathbb{S}^{2}} \sum_{0 \leq i_{1}+i_{2}+i_{3} \leq j}|\not|^{i_{1}} T^{i_{2}}\left(Z^{*}\right)^{i_{3}} \square_{g} \Psi\right|^{2} \sin \theta d t d \theta d \phi<\infty .
\end{gathered}
$$

Remark 5.1.1. Observe that each fixed-r integral is unchanged under the change of variables $t \mapsto t^{*}$ and $\phi \mapsto \phi^{*}$.

\footnotetext{
${ }^{11}$ Recall that in $[30$ the Fourier transform was only applied to Schwartz functions in $t$. The added flexibility gained by working with square integrable functions in $t$ will be crucial for the continuity argument (see Section 11).
} 
5.1.2. The "outgoing" condition. We next introduce an assumption that will imply that solutions of the radial ODE (2) have "outgoing" boundary conditions.

Definition 5.1.2. Let $a_{0}<M$ and $|a| \leq a_{0}$. We shall say a smooth function $\Psi$ is "outgoing" if there exists an $\epsilon>0$ such that $\Psi$ vanishes in $\Sigma_{\tau} \cap\left\{r \leq r_{+}+\epsilon\right\}$ and $\Sigma_{\tau} \cap\left\{r \geq \epsilon^{-1}\right\}$ for all $\tau \leq-\epsilon^{-1}$, and $\square_{g_{a, M}} \Psi$ vanishes for sufficiently large $r$.

We shall see the significance of each of these assumptions individually in Sections 5.2 and 5.3 below. From Section 9 onward, we shall always work in the class defined by assuming both Definitions 5.1.1 and 5.1.2 i.e. $\Psi$ will always be a sufficiently integrable outgoing function.

Remark 5.1.2. Of course, one could significantly weaken the assumptions in Definitions 5.1.1 and 5.1.2; however, this class of functions is very easy to work with, and weakening the assumptions would not simplify the proof of Theorem 3.1.

5.2. Review of Carter's separation. As we have already discussed in our summary of the first parts of the series in Section [1.1.1 we shall view Carter's separation of the wave equation as a convenient geometric framework for frequency-localising energy estimates, closely tied to the Kerr geometry. In the present section, we shall review the relevant formalism from [30].

5.2.1. The oblate spheroidal harmonics. Let $\nu \in \mathbb{R}$. We begin by recalling the collection

$$
\left\{S_{m \ell}(\nu, \cos \theta) e^{i m \phi}\right\}_{m \ell}
$$

of the eigenfunctions of the self-adjoint operator

$$
P(\nu) f=-\frac{1}{\sin \theta} \frac{\partial}{\partial \theta}\left(\sin \theta \frac{\partial}{\partial \theta} f\right)-\frac{\partial^{2} f}{\partial \phi^{2}} \frac{1}{\sin ^{2} \theta}-\nu^{2} \cos ^{2} \theta f
$$

on $L^{2}(\sin \theta d \theta d \phi)$. These form a complete orthonormal basis on $L^{2}(\sin \theta d \theta d \phi)$. The eigenfunctions are parametrised by a set of real discrete eigenvalues $\lambda_{m \ell}^{(\nu)}$

$$
P(\nu) S_{m \ell}(\nu, \cos \theta) e^{i m \phi}=\lambda_{m \ell}(\nu) S_{m \ell}(\nu, \cos \theta) e^{i m \phi},
$$

which have the property that

$$
\begin{gathered}
\lambda_{m \ell}^{(\nu)}+\nu^{2} \geq|m|(|m|+1), \\
\lambda_{m \ell}^{(\nu)}+\nu^{2} \geq 2|m \nu| 12
\end{gathered}
$$

For $\nu=0$ the oblate spheroidal harmonics $S_{m \ell}(\nu, \cos \theta) e^{i m \phi}$ reduce to the standard spherical harmonics $Y_{m \ell}$ with the corresponding eigenvalues $\lambda_{m \ell}^{(0)}=\ell(\ell+1)$.

5.2.2. The coefficients $\Psi_{m \ell}^{(a \omega)}$. Given parameters $a, M$, let $\Psi$ be "sufficiently integrable" in the sense of Definition 5.1.1] We write

$$
\Psi(t, r, \theta, \phi)=\frac{1}{\sqrt{2 \pi}} \int_{-\infty}^{\infty} e^{-i \omega t} \widehat{\Psi}(\omega, r, \theta, \phi) d \omega,
$$

and then, setting $\nu=a \omega$ for each $\omega \in \mathbb{R}$, further decompose

$$
\widehat{\Psi}(\omega, r, \theta, \phi)=\sum_{m \ell} \Psi_{m \ell}^{(a \omega)}(r) S_{m \ell}(a \omega, \cos \theta) e^{i m \phi},
$$

to arrive at

$$
\Psi(t, r, \theta, \phi)=\frac{1}{\sqrt{2 \pi}} \int_{-\infty}^{\infty} \sum_{m \ell} e^{-i \omega t} \Psi_{m \ell}^{(a \omega)}(r) S_{m \ell}(a \omega, \cos \theta) e^{i m \phi} d \omega .
$$

The sufficiently integrable assumption implies that for each $r$, the first and third equality may be interpreted in $L_{t}^{2} L_{\mathbb{S}^{2}}^{2}$, while the second equality may be interpreted in $L_{\omega}^{2} L_{\mathbb{S}^{2}}^{2}$.

\footnotetext{
${ }^{12}$ This follows immediately from the variational characterization of $\lambda_{m \ell}$ and the inequality $\frac{m^{2}}{\sin ^{2} \theta}+\nu^{2} \sin ^{2} \theta \geq 2|m \nu|$.
} 
Furthermore, if $\Psi$ satisfies Definition 5.1.1, then so do $\partial_{t} \Psi, \partial_{\phi} \Psi$ and, by the well-known properties of the Fourier transform, we have

$$
\begin{aligned}
& \partial_{t} \Psi(t, r, \theta, \phi)=\frac{-i}{\sqrt{2 \pi}} \int_{-\infty}^{\infty} \sum_{m \ell} \omega e^{-i \omega t} \Psi_{m \ell}^{(a \omega)}(r) S_{m \ell}(a \omega, \cos \theta) e^{i m \phi} d \omega . \\
& \partial_{\phi} \Psi(t, r, \theta, \phi)=\frac{i}{\sqrt{2 \pi}} \int_{-\infty}^{\infty} \sum_{m \ell} m e^{-i \omega t} \Psi_{m \ell}^{(a \omega)}(r) S_{m \ell}(a \omega, \cos \theta) e^{i m \phi} d \omega .
\end{aligned}
$$

As above, for each $r$ these equalities may be interpreted in $L_{t}^{2} L_{\mathbb{S}^{2}}^{2}$.

Let us take the opportunity to observe the following consequences of Plancherel's formula and the orthonormality of the $S_{m l}(a \omega, \cos \theta) e^{-i m \phi}$ :

$$
\begin{aligned}
& \int_{0}^{2 \pi} \int_{0}^{\pi} \int_{-\infty}^{\infty}|\Psi|^{2}(t, r, \theta, \varphi) \sin \theta d \varphi d \theta d t=\int_{-\infty}^{\infty} \sum_{m \ell}\left|\Psi_{m \ell}^{(a \omega)}(r)\right|^{2} d \omega \\
& \int_{0}^{2 \pi} \int_{0}^{\pi} \int_{-\infty}^{\infty} \Psi \cdot \bar{\Upsilon} \sin \theta d \varphi d \theta d t=\int_{-\infty}^{\infty} \sum_{m \ell} \Psi_{m \ell}^{(a \omega)} \cdot \bar{\Upsilon}_{m \ell}^{(a \omega)} d \omega \\
& \int_{0}^{2 \pi} \int_{0}^{\pi} \int_{-\infty}^{\infty}\left|\partial_{r} \Psi\right|^{2}(t, r, \theta, \varphi) \sin \theta d \varphi d \theta d t=\int_{-\infty}^{\infty} \sum_{m \ell}\left|\frac{d}{d r} \Psi_{m \ell}^{(a \omega)}(r)\right|^{2} d \omega \\
& \int_{0}^{2 \pi} \int_{0}^{\pi} \int_{-\infty}^{\infty}\left|\partial_{t} \Psi\right|^{2}(t, r, \theta, \varphi) \sin \theta d \varphi d \theta d t=\int_{-\infty}^{\infty} \sum_{m \ell} \omega^{2}\left|\Psi_{m \ell}^{(a \omega)}(r)\right|^{2} d \omega .
\end{aligned}
$$

Finally, we note that a straightforward integration by parts, Plancherel and the orthonormality of $S_{m \ell}(a \omega, \cos \theta) e^{i m \phi}$ imply

$$
\begin{aligned}
\int_{0}^{2 \pi} \int_{0}^{\pi} \int_{-\infty}^{\infty} & {\left[\left|\frac{\partial \Psi}{\partial \theta}\right|^{2}+\left|\frac{\partial \Psi}{\partial \phi} \sin ^{-1} \theta\right|^{2}\right](t, r, \theta, \varphi) \sin \theta d \varphi d \theta d t } \\
& =\int_{-\infty}^{\infty} \sum_{m, \ell} \lambda_{m \ell}^{(a \omega)}\left|\Psi_{m \ell}^{(a \omega)}(r)\right|^{2} d \omega-a^{2} \int_{0}^{2 \pi} \int_{0}^{\pi} \int_{-\infty}^{\infty} \cos ^{2} \theta\left|\partial_{t} \Psi\right|^{2} \sin \theta d \varphi d \theta d t
\end{aligned}
$$

5.2.3. The radial ordinary differential equation and the potential $V$. If $\Psi$ is sufficiently integrable in the sense of Definition 5.1.1 define

$$
F=\square_{g} \Psi
$$

for $g=g_{a, M}$ a Kerr metric with $|a|<M$.

The sufficiently integrable assumption implies that we may define the coefficients $\Psi_{m \ell}^{(a \omega)}(r)$, $\left(\rho^{2} F\right)_{m \ell}^{(a \omega)}(r)$ as above.

Carter's formal separation [16] of the wave operator yields:

Proposition 5.2.1. Let $a_{0}<M,|a| \leq a_{0}, \Psi$ be sufficiently integrable, and let $F$ be defined by (40). Then

$$
\Delta \frac{d}{d r}\left(\Delta \frac{d \Psi_{m \ell}^{(a \omega)}}{d r}\right)+\left(a^{2} m^{2}+\left(r^{2}+a^{2}\right)^{2} \omega^{2}-4 M r a \omega m-\Delta\left(\lambda_{m \ell}+a^{2} \omega^{2}\right)\right) \Psi_{m \ell}^{(a \omega)}=\Delta\left(\rho^{2} F\right)_{m \ell}^{(a \omega)}
$$

Note that the sufficiently integrable assumption allows us to interpret this equality for each $r$ in $L_{\omega}^{2} l_{m \ell}^{2}$.

Using the definition (7) of $r^{*}$ and setting

$$
\begin{gathered}
u_{m \ell}^{(a \omega)}(r)=\left(r^{2}+a^{2}\right)^{1 / 2} \Psi_{m \ell}^{(a \omega)}(r), \\
H_{m \ell}^{(a \omega)}(r)=\frac{\Delta\left(\rho^{2} F\right)_{m \ell}^{(a \omega)}(r)}{\left(r^{2}+a^{2}\right)^{3 / 2}},
\end{gathered}
$$


we obtain

$$
\frac{d^{2}}{\left(d r^{*}\right)^{2}} u_{m \ell}^{(a \omega)}+\left(\omega^{2}-V_{m \ell}^{(a \omega)}(r)\right) u=H_{m \ell}^{(a \omega)}
$$

where

$$
V_{m \ell}^{(a \omega)}(r)=\frac{4 M r a m \omega-a^{2} m^{2}+\Delta\left(\lambda_{m \ell}+\omega^{2} a^{2}\right)}{\left(r^{2}+a^{2}\right)^{2}}+\frac{\Delta\left(3 r^{2}-4 M r+a^{2}\right)}{\left(r^{2}+a^{2}\right)^{3}}-\frac{3 \Delta^{2} r^{2}}{\left(r^{2}+a^{2}\right)^{4}} .
$$

In the Schwarzschild case:

$$
\begin{gathered}
V_{m \ell}^{(0 \omega)}(r)=(r-2 M)\left(\frac{\ell(\ell+1)}{r^{3}}+\frac{2 M}{r^{4}}\right) \\
\left(\frac{d V}{d r^{*}}\right)_{m \ell}^{(0 \omega)}(r)=\frac{r-2 M}{r}\left(\frac{2 \ell(\ell+1)(3 M-r)}{r^{4}}+\frac{2 M(8 M-3 r)}{r^{5}}\right) .
\end{gathered}
$$

Again, for each $r$, (44) is to be interpreted in $L_{\omega}^{2} l_{m \ell}^{2}$.

5.2.4. Notational conventions. Following well-established convention, in what follows, as in 30 , we shall suppress the dependence of $u, H$ and $V$ on $a \omega, m, \ell$ in our notation. We will also use the notation

$$
'=\frac{d}{d r^{*}} .
$$

We repeat the following warning from [30]: Since for fixed $g_{a, M}, r$ is a smoothly invertible function of $r^{*}$, we shall often refer to $r^{*}$-ranges by their corresponding $r$-ranges (in particular, given an $r$-parameter such as $R$, we shall very often use the notation $R^{*}=r^{*}(R)$ without further comment), and we shall express functions appearing in most estimates as functions of $r$. The derivative' always is to denote (48)!

5.3. Boundary conditions. In this section, we shall establish boundary conditions for the radial ODE (44). We will require Definitions 5.1.1 and 5.1.2.

Lemma 5.3.1. Let $a_{0}<M,|a| \leq a_{0}$, and $\Psi$ be sufficiently integrable and outgoing. Define $u_{m \ell}^{(a \omega)}(r)$ by (42). Then

$$
\int_{-\infty}^{\infty} \sum_{m \ell}\left|u^{\prime}(r)+i\left(\omega-\frac{a m}{2 M r_{+}}\right) u(r)\right|^{2} d \omega
$$

is a smooth function of $r$ which vanishes at $r=r_{+}$.

Proof. The assumptions on $\Psi$ imply that

$$
\int_{-\infty}^{\infty} \int_{\mathbb{S}^{2}} \sum_{0 \leq i_{1}+i_{2}+i_{3} \leq j}\left|\not{ }^{i_{1}} T^{i_{2}}\left(Z^{*}\right)^{i_{3}} \Psi\right|^{2} \sin \theta d t d \theta d \phi
$$

is a smooth function of $r$. Combining this with the fact that $\partial_{r^{*}}=T+\frac{a}{2 M r_{+}} \Phi$ on $\mathcal{H}^{+}$, we conclude that

$$
\begin{gathered}
\int_{-\infty}^{\infty} \int_{\mathbb{S}^{2}}\left|\partial_{r^{*}}\left(\left(r^{2}+a^{2}\right)^{1 / 2} \Psi\right)-\left(T+\frac{a}{2 M r_{+}}\right)\left(\left(r^{2}+a^{2}\right)^{1 / 2} \Psi\right)\right|^{2} \sin \theta d t d \theta d \phi=O\left(r-r_{+}\right) \Leftrightarrow \\
\int_{-\infty}^{\infty} \sum_{m \ell}\left|u^{\prime}(r)+i\left(\omega-\frac{a m}{2 M r_{+}}\right) u(r)\right|^{2} d \omega=O\left(r-r_{+}\right) \text {as } r \rightarrow r_{+} .
\end{gathered}
$$


Lemma 5.3.2. Let $a_{0}<M,|a| \leq a_{0}$, and let $\Psi$ be sufficiently integrable and outgoing. Define $u_{m \ell}^{(a \omega)}(r)$ by 42). Then, there exists a sequence $\left\{r_{n}\right\}_{n=1}^{\infty}$ such that $r_{n} \rightarrow \infty$ as $n \rightarrow \infty$ and

$$
\lim _{n \rightarrow \infty}\left|\left(u_{m \ell}^{(a \omega)}\right)^{\prime}\left(r_{n}\right)-i \omega u_{m \ell}^{(a \omega)}\left(r_{n}\right)\right| \rightarrow 0
$$

for almost every $\omega$.

Proof. The " $r$-estimate" from 28 with $p=1$, and Definitions 5.1 .1 and 5.1 .2 immediately imply that for $R$ sufficiently large,

$$
\int_{-\infty}^{\infty} \int_{r \geq R} \int_{0}^{2 \pi} \int_{0}^{\pi}\left|\left(\partial_{t}+\partial_{r^{*}}\right)\left(\left(r^{2}+a^{2}\right)^{1 / 2} \Psi\right)\right|^{2} \sin \theta d t d r d \theta d \phi<\infty
$$

An application of Plancherel and a standard pigeonhole argument imply that there exists a dyadic subsequence $\left\{r_{n}\right\}_{n=1}^{\infty}$ such that

$$
\lim _{n \rightarrow \infty} \int_{-\infty}^{\infty}\left|\left(u_{m \ell}^{(a \omega)}\right)^{\prime}\left(r_{n}\right)-i \omega u_{m \ell}^{(a \omega)}\left(r_{n}\right)\right|^{2} d \omega \rightarrow 0 .
$$

Finally, we recall the standard fact that convergence in $L^{2}$ implies almost everywhere pointwise convergence along a subsequence.

As noted in 29] we may formally write these boundary conditions as

$$
\begin{array}{llrl}
u^{\prime}+i\left(\omega-\frac{a m}{2 M r_{+}}\right) u=0, & r & =r_{+}, \\
u^{\prime}-i \omega u=0, & r & =\infty .
\end{array}
$$

5.4. On the almost everywhere regularity of $u_{m \ell}^{(a \omega)}$. The most natural setting for our frequency analysis is the class of functions of $r$ with values in $L_{\omega}^{2} l_{m \ell}^{2}$ referred to already after equality (411). However, for convenience, in Sections 7 and 8 we shall study classical solutions $u$ to the o.d.e. (44). The following lemma will be used in Section 9 to justify the reduction to classical solutions.

Lemma 5.4.1. Let $a_{0}<M,|a| \leq a_{0}$, let $\Psi$ be sufficiently integrable and outgoing, and define $u_{m \ell}^{(a \omega)}(r)$ by (42). Then, for almost every $\omega$, for all $m$ and $\ell, H$ is smooth and $u$ is a smooth solution to the o.d.e. (44) satisfying the boundary conditions (52) and (51).

Proof. Pick any labeling of the eigenvalues $\lambda_{m \ell}$ such that $\lambda_{m \ell}$ is a measurable function $\omega$. Then, using the fact that a countable union of measure zero sets is measure zero, it clearly suffices to prove the lemma for each fixed value of $m$ and $\ell$.

For any $j \geq 1$ and $R>r_{+}$, the fundamental theorem of calculus implies

$$
\begin{aligned}
\sum_{0 \leq i \leq j} \int_{-\infty}^{\infty} \sup _{r \in\left[r_{+}, R\right]}\left|\left(Z^{*}\right)^{i} u\right|^{2} d \omega & \leq \sum_{0 \leq i \leq j} \int_{-\infty}^{\infty} \sum_{m \ell} \sup _{r \in\left[r_{+}, R\right]}\left|\left(Z^{*}\right)^{i} u\right|^{2} d \omega \\
& \leq\left.\sum_{0 \leq i \leq j} \int_{-\infty}^{\infty} \sum_{m \ell}\left|\left(Z^{*}\right)^{i} u\right|^{2}\right|_{r=r_{+}} d \omega+\sum_{0 \leq i \leq j+1} \int_{-\infty}^{\infty} \sum_{m \ell} \int_{r_{+}}^{R}\left|\left(Z^{*}\right)^{i} u\right|^{2} d \omega d r .
\end{aligned}
$$

Next, Plancherel (see the explicit formulas in Section 5.2.2), Sobolev inequalities on $\mathbb{S}^{2}$ and the sufficiently integrable assumption imply that (53) is less than

$$
\begin{gathered}
B \sum_{0 \leq i \leq j+1} \int_{-\infty}^{\infty} \int_{r_{+}}^{R} \int_{\mathbb{S}^{2}}\left|\left(Z^{*}\right)^{i} \Psi\right|^{2} \sin \theta d t^{*} d r d \theta d \phi^{*}+\left.B \sum_{0 \leq i \leq j} \int_{-\infty}^{\infty} \int_{\mathbb{S}^{2}}\left|\left(Z^{*}\right)^{i} \Psi\right|^{2}\right|_{r=r_{+}} \sin \theta d t^{*} d \theta d \phi^{*} \\
\leq B \sum_{0 \leq i \leq j+1, k=0,1,2} \int_{-\infty}^{\infty} \int_{r_{+}}^{R} \int_{\mathbb{S}^{2}}\left|\not^{k}\left(Z^{*}\right)^{i} \Psi\right|^{2} \sin \theta d t^{*} d r d \theta d \phi^{*} \\
+\left.B \sum_{0 \leq i \leq j, k=0,1,2} \int_{\mathbb{S}^{2}} \int_{-\infty}^{\infty}\left|\not^{k}\left(Z^{*}\right)^{i} \Psi\right|^{2}\right|_{r=r_{+}} \sin \theta d t d \theta d \phi^{*}<\infty .
\end{gathered}
$$


Thus, we conclude that for each $n \in \mathbb{Z}_{+}$and $j \geq 0, \sum_{0 \leq i \leq j} \sup _{r \in\left[r_{+}, r_{+}+n\right]}\left|\left(Z^{*}\right)^{i} u\right|^{2}$ is an $L^{2}$ function of $\omega$. Consequently, we may find a set $U_{n}^{(j)} \subset \mathbb{R}$ such that $\left|\left(U_{n}^{(j)}\right)^{c}\right|=0$ and $\omega \in U_{n}^{(j)}$ implies that $u_{m \ell}^{(a \omega)}(r)$ is $C^{j}$ on the interval $\left(r_{+}, r_{+}+n\right)$. Observe that

$$
\left|\left(\cap_{j, n=1}^{\infty} U_{n}^{(j)}\right)^{c}\right|=\left|\cup_{j, n=1}^{\infty}\left(U_{n}^{(j)}\right)^{c}\right| \leq \sum_{j, n=1}^{\infty}\left|\left(U_{n}^{(j)}\right)^{c}\right|=0 .
$$

Thus, we have a set $U \doteq \cap_{j, n=1}^{\infty} U_{n}^{(j)}$ such that the complement of $U$ has measure 0 , and $\omega \in U$ implies that $u_{m \ell}^{(a \omega)}$ is a smooth function of $r$. Of course, the same procedure may be carried out for $H_{m \ell}^{(a \omega)}$. We conclude that for almost every $\omega, u$ and $H$ are smooth functions of $r$, and hence $u$ is a classical solution of the radial o.d.e. (44).

Next, we turn to the boundary condition (52). For every $\omega$ such that $u$ is a classical solution of the radial o.d.e. (44), an asymptotic analysis of the o.d.e. (44) as $r^{*} \rightarrow \infty$ implies that we can find constants $A_{\text {out }}$ and $A_{\text {in }}$ such that

$$
u_{m \ell}^{(a \omega)}=A_{\text {out }} e^{i \omega r^{*}}+A_{\text {in }} e^{-i \omega r^{*}}+O\left(r^{-1}\right) \text { as } r^{*} \rightarrow \infty,
$$

where $O\left(r^{-1}\right)$ is preserved upon differentiation. Lemma 5.3 .2 implies that we must have $A_{\text {in }}=0$, and hence that the boundary condition (52) holds.

Similarly, an asymptotic analysis of the o.d.e. (44) as $r^{*} \rightarrow-\infty$ implies that we can find constants $C_{\text {out }}$ and $C_{\text {in }}$ such that

$$
u_{m \ell}^{(a \omega)}=C_{\text {out }} e^{-i\left(\omega-\omega_{+} m\right) r^{*}}+C_{\text {in }} e^{i\left(\omega-\omega_{+} m\right) r^{*}}+O\left(\left|r^{*}\right|^{-1}\right) \text { as } r^{*} \rightarrow-\infty .
$$

Lemma 5.3.1 implies that we must have $C_{i n}=0$, and hence that the boundary condition (51) holds.

\section{Properties of the potential $V$}

In this section, we prove certain fundamental properties of the potential $V$ appearing in (44), defined by the expression (45). In particular, we shall prove high-frequency regime properties which will be essential for the coercivity of the currents of Section 8 in the high frequency ranges. Sections 6.2 6.4 below follow closely Section 11.1 of our survey [29]. Section 6.5] relevant for the fixed- $m$ case which will be used in our continuity argument of Section 11, is new. Finally, we record explicitly in Section 6.6 the relation of the properties of $V$ proven here to properties of geodesic flow on Kerr.

Remark 6.1. Recall from the outline in Section 1.2 and the discussion of Section 3.4 that the present section, together with Sections 7 and 8 , can be understood to form an independent logical unit of this paper which culminates in Theorem 8.1 giving frequency independent estimates for classical solutions $u$ of the o.d.e. (44) satisfying the boundary conditions (51) and (52). Note that for convenience, this analysis will use the reduction to $a \geq 0$ discussed in Section 4.2. We shall return to the study of (11) in Section 9.

6.1. Admissible frequencies. Recall that the set of eigenvalues $\left\{\lambda_{m \ell}(a \omega)\right\}$ defined by (37) are not known explicitly in closed form. As is clear from (45), the potential depends on $\lambda_{m \ell}(a \omega)$ only through the quantity

$$
\Lambda=\lambda_{m \ell}(a \omega)+a^{2} \omega^{2},
$$

which according to (38) and (39) obeys

$$
\begin{gathered}
\Lambda \geq|m|(|m|+1), \\
\Lambda \geq 2|a m \omega| .
\end{gathered}
$$


It turns out that the results of this section depend only on the constraints (551) and (56), not on the precise values of the set $\left\{\lambda_{m \ell}(a \omega)\right\}$ In what follows, we may thus consider $\omega \in \mathbb{R}, m \in \mathbb{Z}, \Lambda \in \mathbb{R}$ to be independent parameter 13 constrained only by (55) and (56) . This motivates

Definition 6.1.1. We call a frequency triple $(\omega, m, \Lambda)$ admissible if $\omega \in \mathbb{R}, m \in \mathbb{Z}, \Lambda \in \mathbb{R}$, where $\Lambda \geq|m|(|m|+1)$ and $\Lambda \geq 2|a m \omega|$.

6.2. Decomposition of the potential. Given Kerr parameters $0 \leq a<M$, and an admissible frequency triple $(\omega, m, \Lambda)$, we may now define the potential as

$$
V(\omega, m, \Lambda)=V_{0}(\omega, m, \Lambda)+V_{1}
$$

where

$$
\begin{aligned}
& V_{0}=\frac{4 M \operatorname{ram} \omega-a^{2} m^{2}+\Delta \Lambda}{\left(r^{2}+a^{2}\right)^{2}}, \\
& V_{1}=\frac{\Delta\left(3 r^{2}-4 M r+a^{2}\right)}{\left(r^{2}+a^{2}\right)^{3}}-\frac{3 \Delta^{2} r^{2}}{\left(r^{2}+a^{2}\right)^{4}} .
\end{aligned}
$$

Note that $V_{0}$ dominates for high frequencies since $V_{1}$ does not contain any frequency parameters $m$, $\omega, \Lambda$. Note also the nonnegativity property:

$$
V_{1}=\frac{\Delta}{\left(r^{2}+a^{2}\right)^{4}}\left[a^{2} \Delta+2 M r\left(r^{2}-a^{2}\right)\right] \geq 0
$$

6.3. The critical points of $V_{0}$ and the structure of trapping. To understand the nature of trapping, one must first identify the critical points of $V_{0}$. This is provided by the following Lemma. (This appeared as Lemma 11.1.1 of [29]; we repeat its statement and proof here.)

Lemma 6.3.1. Let $M>0, a_{0}<M$ and $0 \leq a \leq a_{0}$. Then for all admissible frequency triples $(\omega, m, \Lambda)$ with $\Lambda>0$, the potential function $V_{0}$ defined by (58) as a function $V_{0}:\left(r_{+}, \infty\right) \rightarrow \mathbb{R}$ is either (a) strictly decreasing, (b) has a unique critical value $r_{\max }^{0}$ which is a global maximum, or (c) has exactly two critical values $r_{\min }^{0}<r_{\max }^{0}$ which are a local minimum and maximum respectively. The value $r_{\max }^{0}$ is bounded independently of the frequency parameters

$$
r_{\max }^{0} \leq B \text {. }
$$

Proof. We have

$$
\begin{aligned}
\frac{d}{d r} V_{0} & =4 m a M \omega\left(\frac{1}{\left(r^{2}+a^{2}\right)^{2}}-\frac{4 r^{2}}{\left(r^{2}+a^{2}\right)^{3}}\right)+\frac{4 r a^{2} m^{2}}{\left(r^{2}+a^{2}\right)^{3}}+\frac{\Lambda}{\left(r^{2}+a^{2}\right)^{2}}\left(2(r-M)-\frac{4 r \Delta}{r^{2}+a^{2}}\right) \\
& =\frac{1}{\left(r^{2}+a^{2}\right)^{3}}\left(4 m a M \omega\left(-3 r^{2}+a^{2}\right)+4 r a^{2} m^{2}-2 \Lambda\left(r^{3}+a^{2} r-3 M r^{2}+M a^{2}\right)\right),
\end{aligned}
$$

and thus,

where we have set

$$
\begin{aligned}
\frac{d}{d r}\left(\left(r^{2}+a^{2}\right)^{3} \frac{d}{d r} V_{0}\right) & =-24 M a m \omega r+4 a^{2} m^{2}-2 \Lambda\left(3 r^{2}-6 M r+a^{2}\right) \\
& =-6 \Lambda\left(r^{2}-2 M r+4 M r \sigma+\frac{a^{2}}{3}-\frac{2}{3} a^{2} \frac{m^{2}}{\Lambda}\right),
\end{aligned}
$$

$\sigma=\frac{a m \omega}{\Lambda}$.

It follows that any critical points of the function $\left(r^{2}+a^{2}\right)^{3} \frac{d}{d r} V_{0}$ must be roots of the quadratic

$$
r^{2}-2 M r(1-2 \sigma)+\frac{a^{2}}{3}\left(1-\frac{2 m^{2}}{\Lambda}\right)
$$

\footnotetext{
${ }^{13}$ In fact, taking $m$ to be integer-valued is of no significance in this analysis, but we will continue to write $m \in \mathbb{Z}$ to avoid confusion.
} 
which we may denote as

$$
r_{1,2}=M(1-2 \sigma) \pm \sqrt{M^{2}(1-2 \sigma)^{2}-\frac{a^{2}}{3}\left(1-\frac{2 m^{2}}{\Lambda}\right)} .
$$

Recalling that $r_{+}>M$, then if $m \omega \geq 0$ (and thus $\sigma \geq 0$ ), it follows that $\operatorname{Re}\left(r_{2}\right)<M$ and thus the only possible critical point on the interval $\left(r_{+}, \infty\right)$ would be

$$
r_{1}=M(1-2 \sigma)+\sqrt{M^{2}(1-2 \sigma)^{2}-\frac{a^{2}}{3}\left(1-\frac{2 m^{2}}{\Lambda}\right)} .
$$

Noting that since $\Lambda>0$, we have

$$
\lim _{r \rightarrow \infty}\left(r^{2}+a^{2}\right)^{3} \frac{d}{d r} V_{0}=-\infty
$$

it follows that $\frac{d}{d r} V_{0}$ either $\left(\mathrm{a}^{*}\right)$ vanishes nowhere, $\left(\mathrm{b}^{*}\right)$ vanishes at a unique point to be denoted $r_{\max }^{0}$, or $\left(\mathrm{c}^{*}\right)$ vanishes at two points, denoted $r_{\min }^{0}<r_{\max }^{0}$, where

$$
\frac{d^{2}}{d r^{2}} V_{0}\left(r_{\min }^{0}\right) \geq 0, \quad \frac{d^{2}}{d r^{2}} V_{0}\left(r_{\max }^{0}\right) \leq 0
$$

In case $\left(\mathrm{a}^{*}\right)$, it follows that $V_{0}$ is strictly decreasing (case (a) of the lemma). In case ( $\left.\mathrm{b}^{*}\right)$, it follows that either $r_{\max }$ is an inflection point and $V_{0}$ is again strictly decreasing (corresponding again to case (a) of the statement of the lemma), or $r_{\max }$ is a global maximum (case (b) of the statement of the lemma). In case $\left(c^{*}\right)$, it is moreover easy to see that these inequalities are in fact strict, and thus $r_{\min }^{0}$ and $r_{\max }^{0}$ correspond to the unique minumum and maximum of $V_{0}$ on $\left(r_{+}, \infty\right)$ (corresponding to case (c) of the statement of the lemma).

If $m \omega<0$ (and thus $\sigma<0$ ), then let us reexpress the root $r_{2}$ by

$$
r_{2}=M(1-2 \sigma)\left(1-\sqrt{1-\frac{a^{2}\left(1-\frac{2 m^{2}}{\Lambda}\right)}{3 M^{2}(1-2 \sigma)^{2}}}\right) .
$$

Since $a<M$ and $\sigma<0$, we have

$$
\frac{a^{2}\left(1-\frac{2 m^{2}}{\Lambda}\right)}{3 M^{2}(1-2 \sigma)^{2}}<\frac{1}{3}
$$

Noting for $0 \leq x<\frac{1}{3}$ the inequality $\sqrt{1-x} \geq 1-\frac{2 x}{3}$, it follows that

$$
\operatorname{Re}\left(r_{2}\right)<\frac{2 M(1-2 \sigma) a^{2}\left(1-\frac{2 m^{2}}{\Lambda}\right)}{9 M^{2}(1-2 \sigma)^{2}}=\frac{2 a^{2}\left(1-\frac{2 m^{2}}{\Lambda}\right)}{9 M(1-2 \sigma)}<\frac{2 M}{9}<r_{+} .
$$

This now implies that $r_{1}$ is the only possible zero of $\frac{d}{d r}\left[\left(r^{2}+a^{2}\right)^{3} \frac{d}{d r} V_{0}\right]$ on the interval $\left[r_{+}, \infty\right)$ and the previous argument applies.

The last statement of the lemma easily follows from observing that for all $\Lambda>0$

$$
\left(r^{2}+a^{2}\right)^{3} \frac{d}{d r} V_{0}=(6 \Lambda M-12 M a m \omega) r^{2}-2 \Lambda r^{3}+O\left(a m \omega, m^{2}\right) r \text { as } r \rightarrow \infty,
$$

and we have $\Lambda \geq|m|(|m|+1), \Lambda \geq 2 a|m \omega|$.

The next statement effectively establishes that even if $r_{\min }^{0}$ exists, it can only be 'trapped' for the value $\omega=\omega_{+} m$. (Again this appeared as Lemma 11.1.2 of [29]. We repeat its statement and proof here.)

Lemma 6.3.2. Let $M>0, a_{0}<M$ and $0 \leq a \leq a_{0}$. For all admissible frequency triples $(\omega, m, \Lambda)$ we have

$$
\omega^{2} \geq V\left(r_{+}\right)
$$


with equality achieved if and only if $\omega=\omega_{+} m$. In particular, in the notations of the previous lemma, this implies that

$$
\omega^{2}>V_{0}\left(r_{\min }^{0}\right)
$$

Proof. We simply compute

$$
\omega^{2}-V\left(r_{+}\right)=\omega^{2}-\frac{4 M r_{+} a m \omega-a^{2} m^{2}}{\left(r_{+}^{2}+a^{2}\right)^{2}}=\frac{\left(2 M r_{+} \omega-a m\right)^{2}}{4 M^{2} r_{+}^{2}} .
$$

Note that the case of equality in (59) occurs precisely at the threshold of the superradiance condition (19):

$$
\omega=\omega_{+} m=\frac{a m}{2 M r_{+}}
$$

6.4. Superradiant frequencies are not trapped. We now turn specifically to the superradiant frequencies, which under the assumption $a \geq 0$ are defined by (19). We will show that these are in fact not trapped, in the sense that, for such frequencies, the maximum of $V$ is always (quantitatively) above the energy level $\omega^{2}$.

First, let us show that for a range of frequency parameters including the superradiant regime, $V_{0}$ can only have a critical point at a maximum, that is the point $r_{\min }^{0}$ is absent. (This was Lemma 11.1.3 of [29] augmented by Remark 11.1.)

Lemma 6.4.1. Let $M>0, a_{0}<M$ and $0 \leq a \leq a_{0}$. Then for all admissible frequency triples $(\omega, m, \Lambda)$ satisfying in addition

$$
m \omega \leq \frac{a m^{2}}{2 M r_{+}}
$$

we have

$$
\frac{d}{d r} V\left(r_{+}\right) \geq \frac{d}{d r} V_{0}\left(r_{+}\right) \geq b \Lambda \geq 0 .
$$

Recall that Lemma 6.3.1 showed that if $r_{\min }^{0}$ exists, we either have $r_{\min }^{0}<r_{\max }^{0}$ or $\frac{d V_{0}}{d r} \leq 0$ on $\left(r_{+}, \infty\right)$. Thus (60) implies that $r_{\min }^{0}$ does not exist and the potential $V_{0}$ has its unique critical point at $r_{\max }^{0}$.

Moreover, for all $\alpha>0$ sufficiently smal14, the same statement holds under the weaker assumption

$$
m \omega \leq \frac{a m^{2}}{2 M r_{+}}+\alpha \Lambda \text {. }
$$

Proof. We begin with the first statement of the lemma. Note

$$
\begin{aligned}
\frac{d}{d r} V_{0}\left(r_{+}\right) & =\frac{4 m a M \omega}{\left(r_{+}^{2}+a^{2}\right)^{3}}\left(-3 r_{+}^{2}+a^{2}\right)+\frac{4 r_{+} a^{2} m^{2}}{\left(r_{+}^{2}+a^{2}\right)^{3}}+\frac{2\left(r_{+}-M\right) \Lambda}{\left(r_{+}^{2}+a^{2}\right)^{2}} \\
& =\frac{1}{\left(r_{+}^{2}+a^{2}\right)^{3}}\left(4 m a M \omega\left(-3 r_{+}^{2}+a^{2}\right)+4 r_{+} a^{2} m^{2}+2\left(r_{+}^{2}+a^{2}\right)\left(r_{+}-M\right) \Lambda\right) .
\end{aligned}
$$

For frequency parameters satisfying $m \omega<0$, the conclusion of the lemma is now obvious, since $-3 r_{+}^{2}+a^{2}<0$. Otherwise, using the condition

$$
0 \leq m \omega \leq \frac{a m^{2}}{2 M r_{+}}
$$

\footnotetext{
${ }^{14}$ Recall our conventions from Section 4.4 on the meaning of this term. This smallness constraint indeed degenerates as $a_{0} \rightarrow M$.
} 
we obtain

$$
\begin{aligned}
\left(r_{+}^{2}+a^{2}\right)^{3} \frac{d}{d r} V_{0}\left(r_{+}\right) & \geq\left(\frac{2 a^{2} m^{2}}{r_{+}}\left(-3 r_{+}^{2}+a^{2}\right)+4 r_{+} a^{2} m^{2}+2\left(r_{+}^{2}+a^{2}\right)\left(r_{+}-M\right) \Lambda\right) \\
& =\left(\frac{2 a^{2} m^{2}}{r_{+}}\left(-r_{+}^{2}+a^{2}\right)+2\left(r_{+}^{2}+a^{2}\right)\left(r_{+}-M\right) \Lambda\right) \\
& =2\left(r_{+}-M\right)\left(\Lambda\left(r_{+}^{2}+a^{2}\right)-2 a^{2} m^{2}\right) \\
& =4\left(r_{+}-M\right)\left(\Lambda M r_{+}-a^{2} m^{2}\right) .
\end{aligned}
$$

The inequalities $\Lambda \geq m^{2}$ and $r_{+}>M>a$ imply that $\frac{d}{d r} V_{0}\left(r_{+}\right) \geq b \Lambda$. We finish the proof of the first statement by recalling that $V=V_{0}+V_{1}$ and observing the identity

$$
\frac{d}{d r} V_{1}\left(r_{+}\right)=\frac{4 M r_{+}\left(r_{+}-M\right)\left(r_{+}^{2}-a^{2}\right)}{\left(r_{+}^{2}+a^{2}\right)^{4}}>0 .
$$

It is clear that the final assertion of the lemma concerning the weaker assumption (61) follows immediately now from the first.

Recall the superradiant condition (19). The statement that superradiant frequencies are not trapped now follows from the following Lemma (again, cf. Lemma 11.1.4 of [29])

Lemma 6.4.2. Let $M>0, a_{0}<M$ and $0 \leq a \leq a_{0}$. For all $\alpha \geq 0$ sufficiently small, then for all admissible frequency triples $(\omega, m, \Lambda)$ satisfying in addition

$$
0<m \omega \leq \frac{a m^{2}}{2 M r_{+}}+\alpha \Lambda
$$

the potential $V_{0}$ satisfies

$$
b \Lambda \leq V_{0}\left(r_{\max }^{0}\right)-\omega^{2} .
$$

Proof. Again, it suffices to prove the lemma with $\alpha=0$. Let $\epsilon>0$ be a fixed sufficiently small constant.

We first consider the case when $m\left(\frac{a m}{2 M r_{+}}-\omega\right) \leq \epsilon|m| \sqrt{\Lambda}$. In this case we have

$$
\omega^{2}-V_{0}\left(r_{+}\right)=\left(\omega-\frac{a m}{2 M r_{+}}\right)^{2} \leq \epsilon^{2} \Lambda .
$$

Combining this with Lemma 6.4.1 easily shows

$$
V_{0}\left(r_{+}+\delta\right)-\omega^{2} \geq b \Lambda
$$

for some sufficiently small $\delta>0$ and even smaller $\epsilon$.

Next, we consider the case when $\omega^{2} \leq \epsilon \Lambda$. Then we clearly have

$$
V_{0}(r)-\omega^{2} \geq \frac{\Lambda}{r^{2}}+O\left(\frac{\Lambda}{r^{3}}\right)-\epsilon \Lambda \text { as } r \rightarrow \infty .
$$

Therefore, if we let $\tilde{r}$ be sufficiently large, and then let $\epsilon$ be sufficiently small, we can arrange for

$$
V_{0}(\tilde{r})-\omega^{2} \geq b \Lambda \text {. }
$$

Finally, we consider the case where $m\left(\frac{a m}{2 M r_{+}}-\omega\right)>\epsilon|m| \sqrt{\Lambda}$ and $\omega^{2}>\epsilon \Lambda$. In this case, $r_{0}:=\frac{a m}{2 M \omega}$ will satisfy $r_{0} \in\left[r_{+}+\delta, R\right]$ for some $\delta>0$ and $R<\infty$. Letting $\Delta_{r_{0}}$ denote $r_{0}^{2}-2 M r_{0}+a^{2}$, we then compute 


$$
\begin{aligned}
\omega^{2}-V_{0}\left(r_{0}\right) & =\omega^{2}-\frac{4 M r_{0} a m \omega-a^{2} m^{2}+\Delta_{r_{0}} \Lambda}{\left(r_{0}^{2}+a^{2}\right)^{2}} \\
& =\frac{1}{\left(r_{0}^{2}+a^{2}\right)^{2}}\left[\left(r_{0}^{2}+a^{2}\right)^{2} \omega^{2}-4 M r_{0} a m \omega+a^{2} m^{2}-\Delta_{r_{0}} \Lambda\right] \\
& =\frac{1}{\left(r_{0}^{2}+a^{2}\right)^{2}}\left[4 M^{2} r_{0}^{2} \omega^{2}-4 M r_{0} a m \omega+a^{2} m^{2}+\omega^{2}\left(\left(r_{0}^{2}+a^{2}\right)^{2}-4 M r_{0}^{2}\right)-\Delta_{r_{0}} \Lambda\right] \\
& =\frac{\omega^{2}\left(r_{0}^{2}-2 M r_{0}+a^{2}\right)\left(r_{0}^{2}+2 M r_{0}+a^{2}\right)-\Delta_{r_{0}} \Lambda}{\left(r_{0}^{2}+a^{2}\right)^{2}} \\
& =\frac{\Delta_{r_{0}}}{\left(r_{0}^{2}+a^{2}\right)^{2}}\left(\frac{a^{2} m^{2}}{4 M^{2}}\left(1+\frac{2 M}{r_{0}}+\frac{a^{2}}{r_{0}^{2}}\right)-\Lambda\right) .
\end{aligned}
$$

We now recall that $a<M<r_{0}$ and that $\Lambda \geq|m|(|m|+1)$ to conclude that

$$
V_{0}\left(r_{0}\right)-\omega^{2} \geq b \frac{\Delta_{r_{0}}}{\left(r_{0}^{2}+a^{2}\right)^{2}} \Lambda \geq b \Lambda .
$$

In the last inequality we have used that $r_{0}$ is bounded away from $r_{+}$and $\infty$ independently of the frequency parameters.

6.5. Trapping for fixed-azimuthal mode solutions. The final result of this section shows in the case of a fixed azimuthal frequency $m$, large $\Lambda$ and $\omega^{2} \sim \Lambda, r_{\max }^{0}$ occurs outside the ergoregion.

Lemma 6.5.1. Let $M>0, a_{0}<M$ and $|a| \leq a_{0}$. Recall that we previously defined $\sigma=\frac{a m \omega}{\Lambda}$. There exists a small constant $c>0$ such that $|\sigma| \leq c, m^{2} \leq c \Lambda$ and $c^{-1} \leq \Lambda$ imply that $r_{\max }^{0}>(1+\sqrt{2}) M$.

Proof. A previous computation showed

$$
\left(r^{2}+a^{2}\right)^{3} \frac{d V_{0}}{d r}=4 m a M \omega\left(-3 r^{2}+a^{2}\right)+4 r a^{2} m^{2}-2 \Lambda\left(r^{3}-3 M r^{2}+a^{2} r+a^{2} M\right) .
$$

Since $r_{\max }^{0}$ is the final critical point of $V_{0}$, we have that $r \geq r_{\max }^{0}$ implies $\frac{d V_{0}}{d r}(r) \leq 0$. Hence, the lemma will follow if we can check that $\frac{d V_{0}}{d r}(r=(1+\sqrt{2}) M)>0$ :

$$
\left.\Lambda^{-1}\left(r^{2}+a^{2}\right)^{3} \frac{d V_{0}}{d r}\right|_{r=(1+\sqrt{2}) M}=O(c)-2\left(M^{3}(1+\sqrt{2})^{3}-3 M^{3}(1+\sqrt{2})^{2}+a^{2} M(1+\sqrt{2})+a^{2} M\right) .
$$

Since we have

we obtain

$$
\begin{aligned}
& (1+\sqrt{2})^{2}=3+2 \sqrt{2}, \\
& (1+\sqrt{2})^{3}=7+5 \sqrt{2},
\end{aligned}
$$

$$
\begin{aligned}
\left.\Lambda^{-1}\left(r^{2}+a^{2}\right)^{3} \frac{d V_{0}}{d r}\right|_{r=(1+\sqrt{2}) M} & =O(c)-2\left(7 M^{3}+5 \sqrt{2} M^{3}-9 M^{3}-6 \sqrt{2} M^{3}+a^{2} M+\sqrt{2} a^{2} M+a^{2} M\right) \\
& =O(c)-2\left(2 M\left(a^{2}-M^{2}\right)+\sqrt{2} M\left(a^{2}-M^{2}\right)\right) .
\end{aligned}
$$

This is positive for sufficiently small $c>0$.

Remark 6.5.1. The importance of the value $r=(1+\sqrt{2}) M$ comes from the fact that this is the unique location of trapping for axisymmetric solutions to the wave equation on an extreme Kerr background, see [6].

Remark 6.5.2. Note that in the case $a=0$, one may drop the assumptions $|\sigma| \leq c$ and $|m|^{2} \leq c \Lambda$ and the $O(c)$ 's which occur in the proof. 
Remark 6.5.3. Of course, the Killing vector field $T$ satisfies

$$
g(T, T)=-\left(\frac{r^{2}-2 M r+a^{2} \cos ^{2} \theta}{r^{2}+a^{2} \cos ^{2} \theta}\right),
$$

which is manifestly negative for $r \geq(1+\sqrt{2}) M>2 M$.

6.6. Aside: relation with null geodesic flow. We note that the potential $V_{0}$ is intimately related to the potential which appears for the radial dependence of solutions of the geodesic equation, i.e. let $\gamma(s)=(t(s), r(s), \theta(s), \phi(s))$ be a null geodesic.

The conserved quantities associated to stationarity and axisymmetry are

$$
\begin{gathered}
E \doteq g(\dot{\gamma}, T)=-\left(1-\frac{2 M r}{\rho^{2}}\right) \dot{t}-\frac{2 M r a \sin ^{2} \theta}{\rho^{2}} \dot{\phi}, \\
L \doteq-g(\dot{\gamma}, \Phi)=\frac{2 M r a \sin ^{2} \theta}{\rho^{2}} \dot{t}-\sin ^{2} \theta \frac{\left(r^{2}+a^{2}\right)^{2}-a^{2} \sin ^{2} \theta \Delta}{\rho^{2}} \dot{\phi} .
\end{gathered}
$$

Carter's hidden conserved quantity is

$$
Q \doteq \rho^{4}(\dot{\theta})^{2}+\frac{L^{2}}{\sin ^{2} \theta}-a^{2} E^{2} \cos ^{2} \theta
$$

Geodesic motion then reduces to the following system (see [15])

$$
\begin{gathered}
\rho^{2} \dot{t}=a\left(E a \sin ^{2} \theta-L\right)+\frac{\left(r^{2}+a^{2}\right)\left(L a-\left(r^{2}+a^{2}\right) E\right)}{\Delta}, \\
\rho^{2} \dot{\phi}=\frac{E a \sin ^{2} \theta-L}{\sin ^{2} \theta}+\frac{a\left(L a-\left(r^{2}+a^{2}\right) E\right)}{\Delta}, \\
\rho^{4}(\dot{\theta})^{2}=Q+a^{2} E^{2}-2 a E L-\frac{\left(L-a E \sin ^{2} \theta\right)^{2}}{\sin ^{2} \theta}, \\
\rho^{4}(\dot{r})^{2}=\left(\left(r^{2}+a^{2}\right) E-a L\right)^{2}-\Delta\left(Q+a^{2} E^{2}-2 a E L\right) .
\end{gathered}
$$

Note that the right hand side of (62) be re-arranged to

$$
\left(r^{2}+a^{2}\right)^{2} E^{2}-4 M a r E L+a^{2} L^{2}-\Delta\left(Q+a^{2} E^{2}\right) .
$$

Under the correspondence $E \mapsto \omega, L \mapsto m$ and $Q \mapsto \lambda_{m l}$, (63) is exactly equal to $\left(r^{2}+a^{2}\right)^{2}\left(\omega^{2}-V_{0}\right)$. Hence, we can write $\dot{r}$ 's equation as

$$
\frac{\rho^{4}}{\left(r^{2}+a^{2}\right)^{2}}(\dot{r})^{2}=E^{2}-V_{0}(E, L, Q, r) .
$$

As a corollary of Lemmas 6.3.1, 6.3.2, 6.4.1 6.4.2 and 6.5.1, one has that (a) null geodesic flow is hyperbolic in a neighborhood of the set of future trapped null geodesics (b) null geodesics $\gamma$ whose future tangent $\dot{\gamma}$ has $g_{a, M}(\dot{\gamma}, T) \geq 0$ are not future trapped; they intersect $\mathcal{H}^{+}$(c) trapped null geodesics orthogonal to $\partial_{\phi}$ lie outside of the ergoregion. We shall not however make direct use of any of these facts at the level of geodesic flow.

\section{The SEPARATED CURRENT TEMPlateS}

Before turning to our estimates we recall the separated current templates of [30] and [29].

\footnotetext{
${ }^{15}$ Instead of $Q$ one often finds the Carter constant defined as $K:=\rho^{4}(\dot{\theta})^{2}+\frac{\left(L-a E \sin ^{2} \theta\right)^{2}}{\sin ^{2} \theta}$, but $Q$ will relate more naturally to our conventions for the wave equation.
} 
7.1. The frequency-localised virial currents $\mathbf{J}^{X, w}$. First, we define the frequency-localised analogue of the virial currents $\mathbf{J}^{X, w}$ where $X$ is in the direction of $\partial_{r^{*}}$, and $w$ is a suitable function.

Fix Kerr parameters $M>0$ and $|a|<M$ and frequency parameters $\omega \in \mathbb{R}, m \in \mathbb{Z}$, and $\Lambda \in \mathbb{R}$. Let $f\left(r^{*}\right), h\left(r^{*}\right)$ and $y\left(r^{*}\right)$ be arbitrary sufficiently regular functions 16 With the notation (48), let us definet the currents

$$
\begin{aligned}
\mathrm{Q}^{f}[u] & =f\left(\left|u^{\prime}\right|^{2}+\left(\omega^{2}-V\right)|u|^{2}\right)+f^{\prime} \operatorname{Re}\left(u^{\prime} \bar{u}\right)-\frac{1}{2} f^{\prime \prime}|u|^{2}, \\
\mathrm{Q}^{h}[u] & =h \operatorname{Re}\left(u^{\prime} \bar{u}\right)-\frac{1}{2} h^{\prime}|u|^{2}, \\
\iota^{y}[u] & =y\left(\left|u^{\prime}\right|^{2}+\left(\omega^{2}-V\right)|u|^{2}\right),
\end{aligned}
$$

associated to the choice of an arbitrary smooth function $u\left(r^{*}\right) 18$

For $u$ satisfying (44), we compute:

$$
\begin{gathered}
\left(\mathrm{Q}^{f}[u]\right)^{\prime}=2 f^{\prime}\left|u^{\prime}\right|^{2}-f V^{\prime}|u|^{2}+\operatorname{Re}\left(2 f \bar{H} u^{\prime}+f^{\prime} \bar{H} u\right)-\frac{1}{2} f^{\prime \prime \prime}|u|^{2}, \\
\left(9^{h}[u]\right)^{\prime}=h\left(\left|u^{\prime}\right|^{2}+\left(V-\omega^{2}\right)|u|^{2}\right)-\frac{1}{2} h^{\prime \prime}|u|^{2}+h \operatorname{Re}(u \bar{H}), \\
\left(h^{y}[u]\right)^{\prime}=y^{\prime}\left(\left|u^{\prime}\right|^{2}+\left(\omega^{2}-V\right)|u|^{2}\right)-y V^{\prime}|u|^{2}+2 y \operatorname{Re}\left(u^{\prime} \bar{H}\right) .
\end{gathered}
$$

The virial currents we shall employ will be various combinations of Q,,, 4 with suitably selected functions $f, h, y$. Note that the choice of these functions may depend on $a, \omega, m, \Lambda$, but, again, we temporarily suppress this from the notation.

7.2. The frequency-localised conserved energy currents. As in our survey [29, we shall need, in addition to the above, a frequency-localised analogue of the conserved energy current $\mathbf{J}^{T}$. Whereas in [29, we introduced also a frequency-localised version of the red-shift current $\mathbf{J}^{N}$, here we shall use in its place a frequency-localised version of the (again conserved) current $\mathbf{J}^{K}$.

Again, fix Kerr parameters $M>0$ and $|a|<M$ and frequency parameters $\omega \in \mathbb{R}, m \in \mathbb{Z}$, and $\Lambda \in \mathbb{R}$. The "frequency-localised" versions of $\mathbf{J}^{T}$ and $\mathbf{J}^{K}$ are then defined as follows:

$$
\begin{aligned}
\mathrm{Q}^{T}[u] & =\omega \operatorname{Im}\left(u^{\prime} \bar{u}\right), \\
\mathrm{Q}^{K}[u] & =\left(\omega-\omega_{+} m\right) \operatorname{Im}\left(u^{\prime} \bar{u}\right),
\end{aligned}
$$

where $\omega_{+}=\frac{a}{2 M r_{+}}$is the "angular velocity" of the event horizon. For $u$ satisfying (44), we have

$$
\begin{gathered}
\left(\mathrm{Q}^{T}[u]\right)^{\prime}=\omega \operatorname{I} m(H \bar{u}), \\
\left(\mathrm{Q}^{K}[u]\right)^{\prime}=\left(\omega-\omega_{+} m\right) \operatorname{Im}(H \bar{u}) .
\end{gathered}
$$

\footnotetext{
${ }^{16}$ In general, $f$ will be bounded and $C^{2}, h$ will be bounded, $C^{1}$ and piecewise $C^{2}$ and $y$ will be bounded, $C^{0}$ and piecewise $C^{1}$.

${ }^{17}$ For better or for worse, we follow here the notation we instituted in the first parts of this series [30. As this notation proved somewhat unpopular, we suggest that readers who dislike archaic Greek simply substitute $\mathrm{Q}^{y}, \mathrm{Q}^{h}$ for both $\zeta^{y}$ and $Q^{h}$, as we shall consistently use functions named $f, h$ and $y$, according to whether we mean $\mathrm{Q}^{f}, \mathrm{Q}^{h}$ or $4^{y}$. Note that in our survey [29], we used the notation $Q_{0}^{f}=\mathrm{Q}^{f}, Q_{1}^{h}=Q^{h}, Q_{2}^{y}=4^{y}$.

${ }^{18}$ Recall that $\mathrm{Q}^{f}$ is itself the combination $Q^{h}[u]+\zeta^{y}[u]$, with $y=f$ and $h=f^{\prime}$, but sufficiently important to deserve its own name!
} 


\section{THE FREQUENCY LOCALISED MULTIPLIER ESTIMATES}

In the present section, using the current templates of Section 7 , we will estimate smooth solutions $u$ to the radial o.d.e. (44) with a general smooth right hand side $H$ and which satisfy the boundary conditions (51) and (52). The point is to obtain estimates which are uniform in the frequency parameters $(\omega, m, \Lambda)$. In view of future applications, we will write the result as an independent theorem. We apply this theorem several times in the present paper (in slightly different contexts) in Sections 9, 11, 12 and 13. We remark that the theorem can in principle be applied in future applications independently of the specific setup of Section 5 .

Before stating the theorem, given $|a| \leq a_{0}<M$, set $R_{-} \doteq r_{+}+\frac{1}{2}\left(r_{\text {red }}-r_{+}\right)$where $r_{\text {red }}$ is the constant from Proposition 4.5.1 and set $R_{+} \doteq 2 R_{\text {large, }}$, where $R_{\text {large }}$ is the constant from Proposition 4.6.1. These values will be referred to below. The precise statement of the main result of this section is

Theorem 8.1. Given $0 \leq a_{0}<M$, there exist positive parameters $\omega_{\mathrm{high}}, \omega_{\mathrm{low}}, \epsilon_{\mathrm{width}}$, $E$ and $R_{\infty}^{*}$, such that the following is true.

Let $0 \leq a \leq a_{0}$ and let $(\omega, m, \Lambda)$ be an admissible frequency triple.

Then there exist functions $f, h, y, \hat{y}, \tilde{y}, \chi_{1}$ and $\chi_{2}$, and a value $r_{\text {trap }}$, depending on the parameters $a_{0}, M, a$ and the frequency triple $(\omega, m, \Lambda)$ but satisfying the uniform bounds

$$
\begin{gathered}
\left|r_{\text {trap }}-r_{+}\right|^{-1}+\left|r_{\text {trap }}\right|+|f|+\Delta^{-1} r^{2}\left|f^{\prime}\right|+|h|+|y|+|\tilde{y}|+|\hat{y}|+\left|\chi_{1}\right|+\left|\chi_{2}\right| \leq B, \\
f+y=1, f^{\prime}=0, h=0,|\tilde{y}| \leq B \exp \left(-b r^{*}\right), \hat{y}=0, \chi_{1}=0, \chi_{2}=1 \text { for } r^{*} \geq R_{\infty}^{*},
\end{gathered}
$$

such that, for all smooth solutions $u$ to the radial o.d.e. (44) with right hand side $H$, satisfying moreover the boundary conditions (51) and (52), we have,

$$
\begin{aligned}
& b \int_{R_{-}^{*}}^{R_{+}^{*}}\left[\left|u^{\prime}\right|^{2}+\left(\left(1-r^{-1} r_{\text {trap }}\right)^{2}\left(\omega^{2}+\Lambda\right)+1\right)|u|^{2}\right] d r^{*} \\
& \leq \int_{-\infty}^{\infty} H \cdot(f, h, y, \chi) \cdot\left(u, u^{\prime}\right) d r^{*}+1_{\left\{\omega_{\text {low }} \leq|\omega| \leq \omega_{\text {high }}\right\} \cap\left\{\Lambda \leq \epsilon_{\text {width }}^{-1} \omega_{\text {high }}^{2}\right\}}|u(-\infty)|^{2} .
\end{aligned}
$$

The symbol $1_{\left\{\omega_{\text {low }} \leq|\omega| \leq \omega_{\text {high }}\right\} \cap\left\{\Lambda \leq \epsilon_{\text {widh }}^{-1} \omega_{\text {high }}^{2}\right\}}$ denotes the indicator function for the set $\left\{\omega_{\text {low }} \leq|\omega| \leq\right.$ $\left.\omega_{\text {high }}\right\} \cap\left\{\Lambda \leq \epsilon_{\text {width }}^{-1} \omega_{\text {high }}^{2}\right\}$, and

$$
\begin{aligned}
H \cdot(f, h, y, \chi) \cdot\left(u, u^{\prime}\right) \doteq & -2 f \operatorname{Re}\left(u^{\prime} \bar{H}\right)-f^{\prime} \operatorname{Re}(u \bar{H})+h \operatorname{Re}(u \bar{H})-E \chi_{2} \omega \operatorname{Im}(H \bar{u}) \\
& -E \chi_{1}\left(\omega-\omega_{+} m\right) \operatorname{Im}(H \bar{u})-2 y \operatorname{Re}\left(u^{\prime} \bar{H}\right)-2 \tilde{y} \operatorname{Re}\left(u^{\prime} \bar{H}\right)-2 \hat{y} \operatorname{Re}\left(u^{\prime} \bar{H}\right) .
\end{aligned}
$$

Before discussing the proof of the theorem, we give a few remarks pertaining to the application of Theorem 8 in Section 9 in the context of $u$ arising from Carter's separation applied to a solution $\Psi$ of the inhomogeneous wave equation.

Remark 8.1. For frequencies in the trapping regime, $r_{\text {trap }}$ will denote the unique trapped value of $r$ associated to the triple $(\omega, m, \Lambda)$. Otherwise, $r_{\text {trap }}$ will be set to 0 . This will capture the degeneration due to trapping.

Remark 8.2. The specific behaviour of the functions $f, h, y, \hat{y}, \tilde{y}, \chi_{1}$ and $\chi_{2}$ in the region $r^{*} \geq R_{\infty}^{*}$ will be useful in Section 9 when we sum (69) to produce a physical space estimate.

Remark 8.3. If we consider the right hand side of the estimate (69) as "data", a direct application of Plancherel (see the explicit formulas in Section 5.2.2) shows that (69) is the phase space versions of integrated local energy decay.

Remark 8.4. Let us draw particular attention to the term $1_{\left\{\omega_{\text {low }} \leq|\omega| \leq \omega_{\text {high }}\right\} \cap\left\{\Lambda \leq \epsilon_{\text {width }}^{-1} \omega_{\text {high }}^{2}\right\}}|u(-\infty)|^{2}$ on the right hand side of the estimate (69). This term must initially be put on the right hand side of the corresponding integrated energy decay statement (cf. Remark 8.3). Eventually, this term will be dealt with in Section 9.7 using the quantitative refinement [59] of mode stability. 
The proof proper of Theorem 8.1 will be given in Section 8.8 It will be based on a series of propositions proven in Sections 8.38 .7 below, where (69) is successively obtained for various ranges of admissible frequency triples. These frequency ranges, however, are determined by parameters which must be suitably optimised so as for our constructions to be possible. We begin thus with a discussion of these ranges and an overview of the constructions.

8.1. The frequency ranges. Let $a_{0}<M$. Fix a parameter $\alpha$ (depending only on $a_{0}, M$ ) satisfying the statement of Lemma 6.4.1. For each $0 \leq a \leq a_{0}$, and all $\omega_{\text {high }}>0, \epsilon_{\text {width }}>0$, we define the frequency ranges $\mathcal{G}_{b}\left(\omega_{\text {high }}\right), \mathcal{G}_{\hbar}\left(\omega_{\text {high }}, \epsilon_{\text {width }}\right), \mathcal{G}_{\mathfrak{\natural}}\left(\omega_{\text {high }}, \epsilon_{\text {width }}\right), \mathcal{G}_{\sharp}\left(\omega_{\text {high }}, \epsilon_{\text {width }}\right), \mathcal{G}^{\sharp}\left(\omega_{\text {high }}, \epsilon_{\text {width }}\right)$ by

- $\mathcal{G}^{\sharp}=\left\{(\omega, m, \Lambda)\right.$ admissible $\left.: \Lambda \geq\left(\frac{a}{2 M r_{+}}+\alpha\right)^{-2} \omega_{\text {high }}^{2}, m \omega \in\left(0, \frac{a m^{2}}{2 M r_{+}}+\alpha \Lambda\right]\right\}$,

- $\mathcal{G}_{\sharp}=\left\{(\omega, m, \Lambda)\right.$ admissible : $\left.|\omega| \geq \omega_{\text {high }}, \Lambda<\epsilon_{\text {width }} \omega^{2}, m \omega \notin\left(0, \frac{a m^{2}}{2 M r_{+}}+\alpha \Lambda\right]\right\}$,

- $\mathcal{G}_{\hbar}=\left\{(\omega, m, \Lambda)\right.$ admissible : $\left.\Lambda \geq \epsilon_{\text {width }}^{-1} \omega_{\text {high }}^{2}, \epsilon_{\text {width }} \Lambda>\omega^{2}, m \omega \notin\left(0, \frac{a m^{2}}{2 M r_{+}}+\alpha \Lambda\right]\right\}$,

- $\mathcal{G}_{\natural}=\left\{(\omega, m, \Lambda)\right.$ admissible : $\left.|\omega| \geq \omega_{\text {high }}, \epsilon_{\text {width }} \Lambda \leq \omega^{2} \leq \epsilon_{\text {width }}^{-1} \Lambda, m \omega \notin\left(0, \frac{a m^{2}}{2 M r_{+}}+\alpha \Lambda\right]\right\}$,

- $\mathcal{G}_{b}=\left\{(\omega, m, \Lambda)\right.$ admissible : $\left.|\omega|<\omega_{\text {high }}, \Lambda<\epsilon_{\text {width }}^{-1} \omega_{\text {high }}^{2}\right\}$.

The parameters $\omega_{\text {high }}$ and $\epsilon_{\text {width }}$ will be fixed in the course of the proof of Theorem 8 , see Section 8.8 . We see easily that

Lemma 8.1.1. With the above notation, for all $0 \leq a \leq a_{0}$ if $(\omega, m, \Lambda)$ is admissible, then, for all choices of parameters $\omega_{\text {high }}, \epsilon_{\text {width }},(\omega, m, \Lambda)$ lies in exactly one of the frequency ranges $\mathcal{G}^{\sharp}, \mathcal{G}_{\sharp}, \mathcal{G}_{\hbar}$, $\mathcal{G}_{\text {古 }}$ or $\mathcal{G}_{\mathrm{b}}$.

Proof. To see this, observe that

$$
|\omega| \geq \omega_{\text {high }} \text { and } m \omega \in\left(0, \frac{a m^{2}}{2 M r_{+}}+\alpha \Lambda\right] \Rightarrow \Lambda \geq\left(\frac{a}{2 M r_{+}}+\alpha\right)^{-2} \omega_{\text {high }}^{2}
$$

Our constructions of currents will vary according to the frequency range of the triple $(\omega, m, \Lambda)$. We now give an overview of these constructions.

8.2. Overview. For each admissible triple $(\omega, m, \Lambda)$, we would like to find a current $\mathrm{Q}$ consisting of various combinations of $\mathrm{Q}^{f}, \mathrm{u}^{y}, \mathrm{Q}^{h}, \mathrm{Q}^{T}$ and $\mathrm{Q}^{K}$ satisfying the bulk coercivity property

$$
\int_{-\infty}^{\infty} \mathrm{Q}^{\prime}[u] \geq b \int_{R_{-}^{*}}^{R_{+}^{*}}\left(\left|u^{\prime}\right|^{2}+\left(1-r^{-1} r_{\text {trap }}\right)^{2}\left(\Lambda+\omega^{2}\right)|u|^{2}+|u|^{2}\right)-\int_{-\infty}^{\infty} H \cdot(f, h, y, \chi) \cdot\left(u, u^{\prime}\right),
$$

and, ideally, the boundary positivity property

$$
\mathrm{Q}(\infty)-\mathrm{Q}(-\infty) \leq 0 \text {. }
$$

The terms Q, $r_{\text {trap }}, H, f, h, y$ should all be understood to depend on $\omega, m$, and $\Lambda$, here omitted for brevity, and the integrals are with respect to $r^{*}$. One restricts the domain of integration on the first term to $\left[R_{-}^{*}, R_{+}^{*}\right]$ on the right hand side because one expects this virial current not to control things at the horizon and infinity.

The most difficult aspect of establishing (71) is the need to understand trapping. In order to do this this we will heavily rely on the analysis of the potential $V_{0}$ carried out in Section 6 . For frequencies for which trapping is relevant, $r_{\text {trap }}$ will denote the unique value of $r$, associated with the frequency triple, where the estimate must degenerate. For frequencies where trapping is not relevant, $r_{\text {trap }}=0$.

The fundamental obstruction to achieving (72), on the other hand, is superradiance (see Section 2.3 .3$)$. For non-superradiant frequencies, i.e. frequencies which satisfy $\omega\left(\omega-\omega_{+} m\right) \geq 0$, one 
may easily 19 control these fluxes via a sufficiently large multiple of the conserved $\mathrm{Q}^{T}$ current:

$$
\int_{-\infty}^{\infty} \operatorname{Im}(H \bar{u})=\int_{-\infty}^{\infty}\left(\mathrm{Q}^{T}\right)^{\prime}=\mathrm{Q}^{T}(\infty)-\mathrm{Q}^{T}(-\infty)=\omega^{2}|u(\infty)|^{2}+\omega\left(\omega-\omega_{+} m\right)|u(-\infty)|^{2} .
$$

However, for superradiant frequencies, where $\omega\left(\omega-\omega_{+} m\right)<0$, no conserved current gives a coercive estimate for the boundary terms and it is thus no longer clear how to arrange for (72). As it turns out, see Section 8.3 below, one of the miracles of the Kerr geometry is that trapping and superradiance are disjoint; exploiting this, one may indeed establish (72) for sufficiently large frequencies with the help of (71) and a large positive parameter. Unfortunately, for bounded superradiant frequencies, one does not have a large parameter at hand. We will not be able to carry out such a scheme, and we will not in fact establish (72); see Section 8.2.5,

We now turn to a more detailed discussion of the difficulties in each frequency range. The reader may wish to refer to this when reading Sections 8.38 .7 below.

8.2.1. The $\mathcal{G} \sharp$ range. This is the large frequency superradiant regime. Lemma6.4.2 shows that these frequencies are not trapped. Thus, it is not difficult to establish (71) via the combination of a $\mathrm{Q}^{f}$ and $\mathrm{Q}^{h}$ current with a monotonically increasing $f$ which switches signs at the unique maximum of the potential and a positive function $h$ which peaks near the maximum of the potential.

As for the boundary terms, despite the lack of a coercive conserved current, we will appeal to the aforementioned miracle that superradiant frequencies are not trapped to find a large parameter which will still allow us to achieve (72). Briefly put, Lemma 6.4.2 shows that we have a quantitatively large "classically forbidden region", and from this one expects to derive an estimate for $u$ near $r_{\max }$ which comes with a large parameter.

8.2.2. The $\mathcal{G}_{\sharp}$ range. This is a non-superradiant regime where the time frequency $\omega$ is large and dominates the other parameters. It is easy to see that a $\iota^{y}$ current with an appropriate choice of $y$ will establish (71).

Of course, the boundary terms may be easily controlled with (73).

8.2.3. The $\mathcal{G}_{\hbar}$ range. This is a non-superradiant regime where the angular frequency $\Lambda$ is large and dominates the other parameters. One may easily show that the conclusions of Lemma 6.4.2 still hold, and, as in Section 8.3. it is not difficult to establish (71).

Turning to the boundary terms, note that $\omega\left(\omega-\omega_{+} m\right)$ and $\left(\omega-\omega_{+} m\right)^{2}$ are not necessarily comparable in this regime. Thus, even though the flux $\left.\mathrm{Q}^{T}[u]\right|_{r=\infty}$ may be controlled with (73), the estimate (73) does not provide sufficient control of the flux $\left.\mathrm{Q}^{K}[u]\right|_{r=r_{+}}$. Fortunately, we may apply the same argument as in the Section 8.3 to control the horizon flux.

8.2.4. The $\mathcal{G}_{h}$ range. This is a non-superradiant regime where the angular frequency $\Lambda$ and the time frequency $\omega$ are large and comparable. This is the regime of trapping and hence the only frequency range where $r_{\text {trap }}$ will be non-zero. The estimate (71) is achieved via a $\mathrm{Q}^{f}$ current with a monotonically increasing function $f$ which switches sign at the unique maximum of the potential. The construction the function $f$ will heavily depend on the critical point analysis of $V_{0}$ carried out in Section 6.3

The estimate (72) is easily achieved via (73).

8.2.5. The $\mathcal{G}_{b}$ range. This is a bounded frequency regime. It turns out to be useful to further split this frequency regime into the following four sub-regimes.

(1) $|\omega| \leq \omega_{\text {low }}, 0 \leq a<\tilde{a}_{0}$ and $m \neq 0$.

(2) $|\omega| \leq \omega_{\text {low }}$ and $m=0$.

(3) $|\omega| \leq \omega_{\text {low }}, m \neq 0$ and $a \geq \tilde{a}_{0}$.

(4) $|\omega| \geq \omega_{\text {low }}$.

\footnotetext{
${ }^{19}$ For the moment we are suppressing the fact that this estimate may be insufficiently strong if $0 \leq \omega\left(\omega-\omega_{+} m\right) \ll$ $\left(\omega-\omega_{+} m\right)^{2}$. See Section 8.2 .3
} 
Here $\omega_{\text {low }}$ is the small parameter mentioned in Theorem 8 and $\tilde{a}_{0}$ is a small parameter to be fixed in the course of the proof.

For the estimate (71) we will exploit two types of estimates. If $|\omega| \geq \omega_{\text {low }}$ or $|\omega| \leq \omega_{\text {low }}, m \neq 0$ and $a \geq \tilde{a}_{0}$, then we will either have $\omega^{2} \sim 1$ or $\left(\omega-\omega_{+} m\right)^{2} \sim 1$. In this case we will employ $\iota^{y}$ currents with exponential multipliers $y \doteq \exp \left(\int v\right)$ and appropriate functions $v$. If $|\omega| \leq \omega_{\text {low }}$ and $\omega_{\text {low }}$ is sufficiently small, then in regions with $1 \lesssim V$ we will have $1 \lesssim V-\omega^{2}$. We will apply $Q^{h}$ currents to exploit this positivity of $V-\omega^{2}$.

As in Section 8.3. the fundamental difficulty is a lack of control of the boundary terms for superradiant frequencies. It turns out that when $\omega^{2} \ll 1$, i.e. $|\omega| \leq \omega_{\text {low }}$ for $\omega_{\text {low }}$ sufficiently small, then $\omega$ arises naturally as a small parameter and we will again be able to achieve (72). However, for bounded frequencies with $|\omega| \geq \omega_{\text {low }}$ there is no large or small parameter to exploit. Instead, for this frequency range we will only be able to establish the weaker

$$
\mathrm{Q}(\infty)-\mathrm{Q}(-\infty) \leq B|u(-\infty)|^{2}
$$

This is the origin of the term $1_{\left\{\omega_{\text {low }} \leq|\omega| \leq \omega_{\text {high }}\right\} \cap\left\{\Lambda \leq \epsilon_{\text {width }}^{-1} \omega_{\text {high }}^{2}\right\}}|u(-\infty)|^{2}$ on the right hand side of the estimate (69).

We now turn to the detailed constructions of the currents for each frequency regime.

8.3. The $\mathcal{G}^{\sharp}$ range. As discussed in Section 8.2.1, this defines a large frequency superradiant regime, and by the results of Section 6.4, frequencies in this regime can be viewed as non-trapped.

Once we have made our final choice of the parameter $\omega_{\text {high }}$, then for $(\omega, m, \Lambda) \in \mathcal{G} \sharp\left(\omega_{\text {high }}\right)$, we will set the functions $y, \hat{y}$ and $\tilde{y}$ together with the parameter $r_{\text {trap }}$ from the statement of Theorem 8.1 to be 0 . The desired coercivity in this range and remaining functions $f, h, \chi_{1}$ and $\chi_{2}$ are given by the following:

Proposition 8.3.1. Let $a_{0}<M$. Then, for all $E \geq 2$, for all $\omega_{\text {high }}$ sufficiently big depending on $E$, for all $R_{\infty}$ sufficiently big, for all $0 \leq a \leq a_{0},(\omega, m, \Lambda) \in \mathcal{G} \sharp\left(\omega_{\text {high }}\right)$, there exist functions $f, h, \chi_{1}$ and $\chi_{2}$ satisfying the uniform bounds

$$
\begin{aligned}
& |f|+\Delta^{-1} r^{2}\left|f^{\prime}\right|+|h|+\left|\chi_{1}\right|+\left|\chi_{2}\right| \leq B\left(\omega_{\text {high }}\right), \\
& f=1, h=0, \chi_{1}=0 \text { and } \chi_{2}=1 \text { for } r \geq R_{\infty},
\end{aligned}
$$

such that, for all smooth solutions $u$ to the radial o.d.e. 44) with right hand side $H$, satisfying moreover the boundary conditions (51) and (52) we have the estimate

$$
\begin{aligned}
& b \int_{R_{-}^{*}}^{R_{+}^{*}}\left(\left|u^{\prime}\right|^{2}+\left(\omega^{2}+\Lambda\right)|u|^{2}\right) \\
& \leq \int_{-\infty}^{\infty}\left(-2 f \operatorname{Re}\left(u^{\prime} \bar{H}\right)-\left(f^{\prime}+h\right) \operatorname{Re}(u \bar{H})+E \chi_{2} \omega \operatorname{Im}(H \bar{u})+E \chi_{1}\left(\omega-\omega_{+} m\right) \operatorname{Im}(H \bar{u})\right) .
\end{aligned}
$$

Proof. As $\mathcal{G} \sharp$ is a superradiant regime with $\Lambda>0$, the conclusions of both Lemma 6.4.1 and Lemma 6.4.2 apply. In particular, the potential $V_{0}$ has a unique $r_{\max }^{0}$ which is a maximum, and satisfies

$$
V_{0}\left(r_{\max }^{0}\right)-\omega^{2} \geq c \Lambda
$$

for some positive constant $c$ depending only on $a_{0}$ and $M$.

We shall first need to establish the following lemma, which shows that the full potential $V$ behaves similarly in the range $\mathcal{G} \sharp\left(\omega_{\text {high }}\right)$ for sufficiently large $\omega_{\text {high }}$.

Lemma 8.3.1. There exists a $\delta>0$ depending only on $a_{0}$ and $M$ such that for sufficiently large $\omega_{\text {high }}$ and $(\omega, m, \Lambda) \in \mathcal{G}^{\sharp}\left(\omega_{\text {high }}\right)$, then $V$ has a unique critical point $r_{\max }$ and satisfies

$$
\begin{gathered}
V(r)-\omega^{2} \geq b \Lambda, \quad \forall r \in\left(r_{\max }-\delta, r_{\max }+\delta\right), \\
-\left(r-r_{\max }\right) \frac{d}{d r} V(r) \geq b \Lambda \frac{\left(r-r_{\max }\right)^{2}}{r^{4}}, \quad \forall r \in\left[r_{+}, \infty\right),
\end{gathered}
$$




$$
\left|r_{\max }-r_{\max }^{0}\right| \leq B \Lambda^{-1}
$$

Proof. Let us first refine our estimates on $V_{0}$ for frequencies $(\omega, m, \Lambda) \in \mathcal{G}^{\sharp}\left(\omega_{\text {high }}\right)$.

Using the fact that $\left|\frac{d V_{0}}{d r}\right| \leq B \Lambda$, (77) implies that we may find a $\delta_{1}>0$ depending only on $a_{0}$ and $M$ such that

$$
V_{0}(r)-\omega^{2} \geq \frac{c}{2} \Lambda, \quad \forall r \in\left[r_{\max }^{0}-\delta_{1}, r_{\max }^{0}+\delta_{1}\right]
$$

Lemma 6.3.1 implies that $r_{\max }^{0}$ is bounded from above independently of the frequency parameters:

$$
r_{\max }^{0} \leq B \text {. }
$$

Furthermore, Lemma 6.4.1 and the bound $\left|\frac{d^{2} V_{0}}{d r^{2}}\right| \leq B \Lambda$ implies $r_{\max }^{0}$ is also bounded away from $r_{+}$ independently of the frequency parameters:

$$
r_{\max }^{0}-r_{+} \geq b
$$

Lemma 6.4.1 also implies that the full potential $V=V_{0}+V_{1}$ satisfies

$$
\frac{d}{d r} V\left(r_{+}\right) \geq \frac{d}{d r} V_{0}\left(r_{+}\right) \geq \hat{c} \Lambda
$$

for a positive constant $\hat{c}$ depending only on $a_{0}$ and $M$.

Recall now that the proof of Lemma 6.3.1 showed that the function

$$
\frac{d}{d r}\left(\left(r^{2}+a^{2}\right)^{3} \frac{d}{d r} V_{0}(r)\right)
$$

is either non-positive on $\left[r_{+}, \infty\right)$ or there exists a unique point $r_{+} \leq r_{1}<r_{\max }^{0}$ such that $\frac{d}{d r}\left(\left(r^{2}+a^{2}\right)^{3} \frac{d}{d r} V_{0}(r)\right)$ is positive on $\left[r_{+}, r_{1}\right)$ and negative on $\left(r_{1}, \infty\right)$.

We first consider the case where the point $r_{1}$ exists. Then,

$$
\frac{d}{d r} V_{0}(r) \geq \hat{c} \frac{\left(r_{+}^{2}+a^{2}\right)^{3}}{\left(r_{1}^{2}+a^{2}\right)^{3}} \Lambda \quad \forall r \in\left[r_{+}, r_{1}\right] .
$$

Next, recall from the proof of Lemma 6.3.1 that

$$
\frac{d}{d r}\left(\left(r^{2}+a^{2}\right)^{3} \frac{d V_{0}}{d r}\right)=-6 \Lambda\left(r^{2}-2 M r+4 M r \sigma+\frac{a^{2}}{3}-\frac{2}{3} a^{2} \frac{m^{2}}{\Lambda}\right),
$$

and furthermore, by definition, $\frac{d}{d r}\left(\left(r^{2}+a^{2}\right)^{3} \frac{d V_{0}}{d r}\right)$ is negative on $\left(r_{1}, \infty\right)$. Thus, we can choose a value $r_{1}^{\prime} \in\left(r_{1}, r_{\max }^{0}\right)$ such that

$$
\frac{d}{d r} V_{0}(r) \geq \frac{\hat{c}}{2} \frac{\left(r_{+}^{2}+a^{2}\right)^{3}}{\left(r_{1}^{2}+a^{2}\right)^{3}} \Lambda, \quad \forall r \in\left[r_{+}, r_{1}^{\prime}\right]
$$

and

$$
\frac{d}{d r}\left(\left(r^{2}+a^{2}\right)^{3} \frac{d}{d r} V_{0}(r)\right) \leq-\tilde{c} \Lambda r^{2}, \quad \forall r \in\left[r_{1}^{\prime}, \infty\right),
$$

for a positive constant $\tilde{c}$ independent of the frequency parameters.

In the case where $r_{1}$ does not exists, the same argument mutatis mutandis will produce a value $r_{1}^{\prime}$ with the properties (80) and (81).

Now, we simply observe that the potential $V_{1}$ satisfies the bounds

$$
\left|V_{1}\right| \leq B r^{-3}, \quad\left|\frac{d}{d r} V_{1}(r)\right| \leq B r^{-4}, \quad\left|\frac{d}{d r}\left(\left(r^{2}+a^{2}\right)^{3} \frac{d}{d r} V_{1}(r)\right)\right| \leq B r .
$$

For $\omega_{\text {high }}$ sufficiently large (and hence large $\Lambda$ ), it immediately follows that, for $(\omega, m, \Lambda) \epsilon$ $\mathcal{G} \sharp\left(\omega_{\text {high }}\right)$, the full potential $V=V_{0}+V_{1}$ cannot have any critical points on $\left[r_{+}, r_{1}^{\prime}\right]$ and has a unique maximum $r_{\max } \in\left[r_{1}^{\prime}, \infty\right)$ which satisfies $\left|r_{\max }-r_{\max }^{0}\right| \leq B \Lambda^{-1}$.

The proof concludes by applying the fact that $\left|\frac{d V}{d r}\right| \leq B \Lambda$. 
We now proceed to the construction of a suitable current for the regime $\mathcal{G} \sharp$. The current will be of the form:

$$
\mathrm{Q}=\mathrm{Q}^{f}+\mathrm{Q}^{h}-E \chi_{1} \mathrm{Q}^{K}-E \chi_{2} \mathrm{Q}^{T}
$$

for appropriate functions $f, h, \chi_{1}$ and $\chi_{2}$ and large constant $E$.

It is simpler to describe this procedure in three stages.

Stage 1. We first apply current $\mathrm{Q}^{f}$ where $f$ is a function chosen such that

$$
\begin{gathered}
f=-1 \text { at } r=r_{+}, \quad f=0 \text { at } r=r_{\max }, \quad f=1 \text { when } r^{*} \geq R_{\infty}^{*}, \\
f^{\prime}\left(r^{*}\right)>0 \text { for all } r \leq R_{1}, \quad f^{\prime}\left(r^{*}\right) \geq 0 \text { for all } r>r_{+}, \quad|f|+\Delta^{-1} r^{2}\left|f^{\prime}\right| \leq B .
\end{gathered}
$$

Application of (64) yields then

$$
\begin{gathered}
\int_{-\infty}^{\infty}\left(2 f^{\prime}\left|u^{\prime}\right|^{2}-f V^{\prime}|u|^{2}-\frac{1}{2} f^{\prime \prime \prime}|u|^{2}\right)=\left(\left|u^{\prime}\right|^{2}+\left(\omega-\omega_{+} m\right)^{2}|u|^{2}\right)_{r=r_{+}}+\left(\left|u^{\prime}\right|^{2}+\omega^{2}|u|^{2}\right)_{r=\infty} \\
-\int_{-\infty}^{\infty}\left(2 f \operatorname{Re}\left(u^{\prime} \bar{H}\right)+f^{\prime} \operatorname{Re}(u \bar{H})\right) .
\end{gathered}
$$

Let us moreover require that $f$ above has been chosen so that in addition to (82), (83), the following coercivity property holds

$$
-f V^{\prime}-\frac{1}{2} f^{\prime \prime \prime} \geq \Lambda \frac{\Delta\left(r-r_{\max }\right)^{2}}{r^{7}}, \text { for all } r>r_{+} .
$$

Since $f$ vanishes at $r=r_{\max }$ and $V^{\prime}$ obeys the property (78), we can easily arrange such that in addition to (82), (83) and (85), we have

$$
f V^{\prime} \geq b \Lambda \frac{\Delta\left(r-r_{\max }\right)^{2}}{r^{7}} .
$$

It remains to impose

$$
f^{\prime \prime \prime}(r)<0 \text { in a small neighbourhood of } r_{\max }, \quad\left|f^{\prime \prime \prime}(r)\right| \leq B \Delta r^{-5} .
$$

Note that the reader may easily construct a function $f$ satisfying the conditions (82), (83), (85), (86) and (87). With the above choice of $f$, the left hand side of (84) is now non-negative, but still degenerate at $r=r_{\max }$. As discussed in Section 6.4 the bound $V\left(r_{\max }\right)-\omega^{2} \geq b \Lambda$ indicates this regime is non-trapped and thus the degeneracy may be removed with the help of the current $Q^{h}$. The more serious problem is a lack of control of the boundary terms on the right hand side, due to the superradiant condition. However, as we shall see below, we will be able to overcome this by exploiting the largeness of the potential in the region $\left(r_{\max }-\delta, r_{\max }+\delta\right)$.

Stage 2. We now add a $Q^{h}$ current with a function $h \doteq A \tilde{h}$ such that

$$
\begin{aligned}
h \geq 0, \quad|\tilde{h}| \leq B, \\
\operatorname{supp}(h) \subset\left[r_{\max }-\delta, r_{\max }+\delta\right], \quad \tilde{h}=1 \text { for } r \in\left[r_{\max }-\delta / 2, r_{\max }+\delta / 2\right]
\end{aligned}
$$

and $A$ is a constant to be determined.

We obtain

$$
\begin{aligned}
& \int_{-\infty}^{\infty}\left(\left(2 f^{\prime}+A h\right)\left|u^{\prime}\right|^{2}+\left(A \tilde{h}\left(V-\omega^{2}\right)-f V^{\prime}\right)|u|^{2}-\frac{1}{2}\left(f^{\prime \prime \prime}+A h^{\prime \prime}\right)|u|^{2}\right) \\
& (90) \quad=\left(\left|u^{\prime}\right|^{2}+\left(\omega-\omega_{+} m\right)^{2}|u|^{2}\right)_{r=r_{+}}+\left(\left|u^{\prime}\right|^{2}+\omega^{2}|u|^{2}\right)_{r=\infty}-\int_{-\infty}^{\infty}\left(2 f \operatorname{Re}\left(u^{\prime} \bar{H}\right)+\left(f^{\prime}+h\right) \operatorname{Re}(u \bar{H})\right) .
\end{aligned}
$$

Note that as long as $A \leq \tilde{\epsilon} \omega_{\text {high }}^{2}$ for a sufficiently small constant $\tilde{\epsilon}$ only depending on $a_{0}$ and $M$, the integrand of the left hand side of (90) will be positive. Moreover, this integrand has the property that it satisfies

$$
\geq b A\left(\left|u^{\prime}\right|^{2}+\Lambda|u|^{2}\right), \quad \forall r \in\left[r_{\max }-\frac{\delta}{2}, r_{\max }+\frac{\delta}{2}\right] .
$$


Stage 3. We now let $\chi_{1}(r)$ be a smooth function such that

$$
\chi_{1}=1 \text { for } r \in\left[r_{+}, r_{\max }-\frac{\delta}{2}\right], \quad \chi_{1}=0 \text { for } r \in\left[r_{\max }+\frac{\delta}{2}, \infty\right), \quad\left|\chi_{1}\right| \leq B .
$$

Since $E \geq 2$, we have

$$
\begin{aligned}
\left(\left|u^{\prime}\right|^{2}+\left(\omega-\omega_{+} m\right)^{2}|u|^{2}\right)_{r=r_{+}} & \leq E \int_{-\infty}^{\infty}\left(\chi_{1} \mathrm{Q}^{K}\right)^{\prime} \\
& =E \int_{r_{\max }-\frac{\delta}{2}}^{r_{\max }+\frac{\delta}{2}} \chi_{1}^{\prime}\left(\omega-\omega_{+} m\right) \operatorname{Im}\left(u^{\prime} \bar{u}\right)+E \int_{-\infty}^{\infty} \chi_{1}\left(\omega-\omega_{+} m\right) \operatorname{Im}(H \bar{u})
\end{aligned}
$$

Now, we require that $\omega_{\text {high }}$ be sufficiently large so as to satisfy $E \delta^{-1} \ll(1 / 2) \tilde{\epsilon} \omega_{\text {high }}^{2}$, and then we set $A \doteq(1 / 2) \tilde{\epsilon} \omega_{\text {high }}^{2}$. This choice of $A$ will both maintain the coercivity of the left hand side of (90) and yield

$$
\begin{aligned}
E\left|\int_{r_{\max }-\frac{\delta}{2}}^{r_{\max }+\frac{\delta}{2}} \chi_{1}^{\prime}\left(\omega-\omega_{+} m\right) \operatorname{Im}\left(u^{\prime} \bar{u}\right)\right| & \leq E \delta^{-1} \int_{r_{\max }-\frac{\delta}{2}}^{r_{\max }+\frac{\delta}{2}}\left(\left|u^{\prime}\right|^{2}+\left(\omega^{2}+m^{2}\right)|u|^{2}\right) \\
& \ll A \int_{r_{\max }-\frac{\delta}{2}}^{r_{\max }+\frac{\delta}{2}}\left(\left|u^{\prime}\right|^{2}+\Lambda|u|^{2}\right) .
\end{aligned}
$$

We can, of course, carry out an analogous construction with a cutoff $\chi_{2}$, satisfying

$$
\chi_{2}=1 \text { for } r \in\left[r_{\max }+\frac{\delta}{2}, \infty\right), \quad \chi_{2}=0 \text { for } r \in\left[r_{+}, r_{\max }-\frac{\delta}{2}\right], \quad\left|\chi_{2}\right| \leq B,
$$

and the current $\mathrm{Q}^{T}$. Then, adding the currents $-E \chi_{1} \mathrm{Q}^{K}-E \chi_{2} \mathrm{Q}^{T}$ will give us the necessary control of the boundary terms.

Observing that the left hand side of the resulting estimate is coercive (with weights which degenerate however as $r^{*} \rightarrow \pm \infty$ ), restricting the domain of integration of the left hand side then yields (75).

8.4. The $\mathcal{G}_{\sharp}$ range. As discussed in Section 8.2.2, $\mathcal{G}_{\sharp}$ defines a large frequency regime (whose definition still depends on parameters $\omega_{\text {high }}$ and $\epsilon_{\text {width }}$, yet to be fixed) where time frequencies will dominate angular frequencies. The regime is manifestly non-superradiant, and, for suitable choice of parameters, non-trapped.

Once we have made our final choice of parameters $\omega_{\text {high }}$ and $\epsilon_{\text {width }}$, then for $(\omega, m, \Lambda) \in \mathcal{G}_{\sharp}\left(\omega_{\text {high }}, \epsilon_{\text {width }}\right)$, we will set the functions $f, h, \hat{y}, \tilde{y}$ and $\chi_{1}$ appearing in Theorem 8.1 together with the parameter $r_{\text {trap }}$ to be 0 . The remaining function $y$ and the desired coercivity property are given by

Proposition 8.4.1. Let $a_{0}<M$. Then, for all $\omega_{\text {high }}, \epsilon_{\text {width }}^{-1}, R_{\infty}$ sufficiently big, for all $E \geq 2$, $0 \leq a \leq a_{0},(\omega, m, \Lambda) \in \mathcal{G}_{\sharp}\left(\omega_{\text {high }}, \epsilon_{\text {width }}\right)$, there exists a function $y$ satisfying the uniform bounds

$$
\begin{gathered}
|y| \leq B, \\
y=1 \text { for } r^{*} \geq R_{\infty}^{*},
\end{gathered}
$$

such that, for all smooth solutions $u$ to the radial o.d.e. (44) with right hand side H, satisfying moreover the boundary conditions (51) and (52) we have the estimate

$$
b \int_{R_{-}^{*}}^{R_{+}^{*}}\left(\left|u^{\prime}\right|^{2}+\left(\omega^{2}+\Lambda\right)|u|^{2}\right) \leq \int_{-\infty}^{\infty}\left(-2 y \operatorname{Re}\left(u^{\prime} \bar{H}\right)+E \omega \operatorname{Im}(H \bar{u})\right) .
$$

Proof. The construction of our currents will exploit the fact that the range $\mathcal{G}_{\sharp}$ defines a large frequency regime in which $\Lambda \ll \omega^{2}$ (and thus also $m^{2} \ll \omega^{2}$ ). To handle the boundary terms, we will use that this regime is moreover manifestly non-superradiant, and thus addition of a sufficiently large multiple of the $\mathrm{Q}^{T}$ current provides positive terms at $r=r_{+}$and $r=\infty$. 
We turn to the details. First of all, it is easy to see that the admissibility inequalities $\Lambda \geq 2 a|m \omega|$ and $\Lambda \geq|m|(|m|+1)$ imply that there exists a constant $R_{\mathrm{dec}}^{*} \geq 2 R_{+}^{*}$ only depending on $a_{0}$ and $M$ such that

$$
V^{\prime}<0 \text { for } r^{*} \geq R_{\mathrm{dec}}^{*}
$$

Define a current given by the following expression:

$$
\mathrm{Q}=\iota^{y}-E \mathrm{Q}^{T} .
$$

We require that

$$
\begin{gathered}
\left|y^{\prime}\right| \leq B, \quad y^{\prime} \geq 0 \text { for } r \in\left[r_{+}, \infty\right), \quad y^{\prime}>0 \text { for } r^{*} \in\left[R_{-}^{*}, R_{1}^{*}\right], \\
\frac{1}{2} \leq y \leq 1 \text { for } r^{*} \in\left(-\infty, R_{+}^{*}\right], \quad y(-\infty)=1 / 2, \quad y=1 \text { for } r^{*} \geq R_{\mathrm{dec}}^{*} .
\end{gathered}
$$

Such a $y$ is trivial to construct.

We obtain from (66) and (67) the identity

$$
\begin{aligned}
& \int_{-\infty}^{\infty}\left(y^{\prime}\left|u^{\prime}\right|^{2}+\left(y^{\prime}\left(\omega^{2}-V\right)-y V^{\prime}\right)|u|^{2}\right) \\
& \quad-\left(\frac{1}{2}\left|u^{\prime}\right|^{2}+\left(\frac{1}{2}\left(\omega-\omega_{+} m\right)^{2}-E \omega\left(\omega-\omega_{+} m\right)\right)|u|^{2}\right)_{r=r_{+}}-\left(\left|u^{\prime}\right|^{2}+(1-E) \omega^{2}|u|^{2}\right)_{r=\infty} \\
& =\int_{-\infty}^{\infty}\left(-2 y \operatorname{Re}\left(u^{\prime} \bar{H}\right)+E \omega \operatorname{Im}(\bar{H} u)\right) .
\end{aligned}
$$

Next, we observe the bound

$$
\left.\left.|V| \leq B\left(\epsilon^{-1} \Lambda+\epsilon \omega^{2}+\omega_{\text {high }}^{-2}\right) \omega^{2}\right), \quad\left|V^{\prime}\right| \leq \frac{B \Delta}{r^{5}}\left(\epsilon^{-1} \Lambda+\epsilon \omega^{2}+\omega_{\text {high }}^{-2}\right) \omega^{2}\right)
$$

where $\epsilon>0$ is arbitrary.

Now, we fix a sufficiently small $\epsilon>0$, require that $\epsilon_{\text {width }}$ is sufficiently small depending on $\epsilon$, and combine the inequality $\omega^{2}>\epsilon_{\text {width }}^{-1} \Lambda$ with the inequalities (98) and (94). We conclude the integrand on the left hand side of (97) is non-negative and bounds from above the expression

$$
b \int_{R_{-}^{\star}}^{R_{+}^{*}}\left(\left|u^{\prime}\right|^{2}+\left(\omega^{2}+\Lambda\right)|u|^{2}\right) .
$$

The boundary terms are non-negative due to the boundary conditions (51) and (52), the nonsuperradiance condition and the requirement $E \geq 2$. Requiring that $R_{\infty}^{*}>R_{\mathrm{dec}}^{*}$ ensures that (93) is satisfied.

8.5. The $\mathcal{G}_{\phi}$ range. As described in Section 8.2.3, this is again a large frequency regime (whose definition still depends on parameters $\omega_{\text {high }}$ and $\epsilon_{\text {width }}$ yet to be fixed), but where angular frequencies will now dominate time frequencies. The regime is again manifestly non-superradiant, and, for suitable parameters, non-trapped, but as we shall see, we will have to handle the horizon boundary term as in the superradiant regime.

Once we have made our final choice of the parameters $\omega_{\text {high }}$ and $\epsilon_{\text {width }}$, then for $(\omega, m, \Lambda) \epsilon$ $\mathcal{G}_{\hbar}\left(\omega_{\text {high }}, \epsilon_{\text {width }}\right)$, we set the functions $y, \tilde{y}$ and $\hat{y}$ together with the parameter $r_{\text {trap }}$ to be 0 . The remaining functions $f, h$ and $\chi_{1}$ and the desired coercivity properties are given by

Proposition 8.5.1. Let $a_{0}<M$. Then, for all $\omega_{\text {high }}, R_{\infty}$ and $\epsilon_{\text {width }}^{-1}$ sufficiently large, for all $E \geq 2$, $0 \leq a \leq a_{0},(\omega, m, \Lambda) \in \mathcal{G}_{\sharp}\left(\omega_{\text {high }}, \epsilon_{\mathrm{width}}\right)$, there exist functions $f, h$ and $\chi_{1}$ satisfying the uniform bounds

$$
\begin{gathered}
|f|+\Delta^{-1} r^{2}\left|f^{\prime}\right|+|h|+\left|\chi_{1}\right|+\left|\chi_{2}\right| \leq B\left(\omega_{\text {high }}, \epsilon_{\text {width }}\right), \\
f=1, h=0, \chi_{1}=0 \text { for } r^{*} \geq R_{\infty}^{*}
\end{gathered}
$$


such that, for all smooth solutions $u$ to the radial o.d.e. (44) with right hand side $H$, satisfying moreover the boundary conditions (51) and (52), we have the estimate

$$
\begin{aligned}
& b \int_{R_{-}^{*}}^{R_{+}^{*}}\left(\left|u^{\prime}\right|^{2}+\left(\omega^{2}+\Lambda\right)|u|^{2}\right) \\
& \quad \leq \int_{-\infty}^{\infty}\left(-2 f \operatorname{Re}\left(u^{\prime} \bar{H}\right)-\left(f^{\prime}+h\right) \operatorname{Re}(u \bar{H})+E \omega \operatorname{Im}(H \bar{u})+\chi_{1}\left(\omega-\omega_{+} m\right) \operatorname{Im}(H \bar{u})\right) .
\end{aligned}
$$

Proof. For the construction of our currents, we again shall exploit that $\mathcal{G}_{\hbar}$ defines a large frequency regime, where now, however, $\omega^{2} \ll \Lambda$. Since this is a non-superradiant regime, the boundary term of $r^{*}=\infty$ may be controlled with the $\mathrm{Q}^{T}$ current; however, we shall handle the boundary term at the horizon as we did for the regime $\mathcal{G}^{\sharp}$. As we explained in Section 8.2.3 this is necessary because the boundary term at the horizon is proportional to $\left(\omega-\omega_{+} m\right)^{2}|u(-\infty)|^{2}$, and the $\mathrm{Q}^{T}$ current would only give an estimate for $\omega\left(\omega-\omega_{+} m\right)|u(-\infty)|^{2}$. In the frequency regime under consideration these are not necessarily comparable.

Turning to the proof, we begin by arguing that $\epsilon_{\text {width }}$ sufficiently small implies $m \omega \leq 0$. Suppose $m \omega>0$. Then we have

$$
m \omega \geq \frac{a m^{2}}{2 M r_{+}}+\alpha \Lambda \geq \alpha \epsilon_{\text {width }}^{-1} \omega^{2} \Rightarrow|m| \geq \alpha \epsilon_{\text {width }}^{-1}|\omega| .
$$

On the other hand,

$$
m \omega \geq \frac{a m^{2}}{2 M r_{+}}+\alpha \Lambda \Rightarrow|\omega| \geq \alpha \Lambda|m|^{-1} \geq \alpha|m| .
$$

Combining (99) and (100) implies

$$
|\omega| \geq \alpha^{2} \epsilon_{\text {width }}^{-1}|\omega| .
$$

This is a contradiction if we take $\epsilon_{\mathrm{width}}<\alpha^{2}$. Thus, we indeed have $m \omega<0$.

From the above inequality, it follows that Lemma 6.4.1 applies, and we may thus conclude that the potential $V_{0}$ is increasing at $r_{+}$, and hence has only one critical point at $r=r_{\max }^{0}$ where it attains a maximum. As in the proof of Proposition 8.3 .1 concerning the regime $\mathcal{G}^{\sharp}$, we again infer that, for $\omega_{\text {width }}$ sufficiently large, in the regime $\mathcal{G}_{\hbar}\left(\omega_{\text {high }}, \epsilon_{\text {width }}\right)$, the potential $V$ has a unique non-degenerate critical point at $r_{\max }$, where it attains a maximum, and that $r_{\max }$ is uniformly bounded away from $r_{+}$and is uniformly bounded from above. Similarly, we also obtain the existence of an interval $\left(r_{\max }-\delta, r_{\max }+\delta\right)$, where $\delta$ is independent of frequency parameters, such that $V$ satisfies the two relations

$$
V(r)-\omega^{2} \geq b \Lambda, \quad \forall r \in\left(r_{\max }-\delta, r_{\max }+\delta\right)
$$

and

$$
\left(r-r_{\max }\right) \frac{d}{d r} V(r) \geq b \Lambda \frac{\left(r-r_{\max }\right)^{2}}{r^{4}}, \quad r>r_{+} .
$$

We may now follow the construction given in Proposition 8.3.1 for the range $\mathcal{G} \sharp$. We define first a current $\mathrm{Q}=\mathrm{Q}^{f}+\mathrm{Q}^{h}$ with the same choice of functions $f$ and $h=A \tilde{h}$ as for $\mathcal{G} \sharp$. This gives the inequality

$$
\begin{gathered}
\int_{-\infty}^{\infty}\left(\left(2 f^{\prime}+A h\right)\left|u^{\prime}\right|^{2}+\left(A h\left(V-\omega^{2}\right)-f V^{\prime}\right)|u|^{2}-\frac{1}{2}\left(f^{\prime \prime \prime}+A h^{\prime \prime}\right)|u|^{2}\right) \\
=\left(\left|u^{\prime}\right|^{2}+\left(\omega-\omega_{+} m\right)^{2}|u|^{2}\right)_{r=r_{+}}+\left(\left|u^{\prime}\right|^{2}+\omega^{2}|u|^{2}\right)_{r=\infty} \\
\quad-\int_{-\infty}^{\infty}\left(2 f \operatorname{Re}\left(u^{\prime} \bar{H}\right)+\left(f^{\prime}+A h\right) \operatorname{Re}(u \bar{H})\right),
\end{gathered}
$$

where the integrand on the left hand side is positive definite. As in the $\mathcal{G}^{\sharp}$ regime, we may gain a large parameter in the region $\left(r_{\max }-\delta, r_{\max }+\delta\right)$ by observing that there exists a small constant $\tilde{\delta}$ only depending on $a_{0}$ and $M$ so that, as long as $A \leq \tilde{\delta} \epsilon_{\text {width }}^{-1} \omega_{\text {high }}^{2}$, the left-hand side of (101) will give 
a coercive estimate. We fix such an $A$. Finally, using a $\chi_{1}\left(\omega-\omega_{+} m\right) \mathrm{Q}^{K}$ current we may handle the boundary term at the horizon, mutatis mutandis, as we did for the $\mathcal{G} \sharp$ regime. We obtain

$$
\begin{aligned}
b \int_{-\infty}^{\infty} & \left(\left(2 f^{\prime}+A h\right)\left|u^{\prime}\right|^{2}+\left(A h\left(V-\omega^{2}\right)-f V^{\prime}\right)|u|^{2}-\frac{1}{2}\left(f^{\prime \prime \prime}+A h^{\prime \prime}\right)|u|^{2}\right) \\
& \leq\left(\left|u^{\prime}\right|^{2}+\omega^{2}|u|^{2}\right)_{r=\infty}-\int_{-\infty}^{\infty}\left(2 f \operatorname{Re}\left(u^{\prime} \bar{H}\right)+\left(f^{\prime}+A h\right) \operatorname{Re}(u \bar{H})+\chi_{1}\left(\omega-\omega_{+} m\right) \operatorname{Im}(H \bar{u})\right) .
\end{aligned}
$$

Finally, for any $E \geq 2$, the boundary term at infinity is controlled easily with a $\mathrm{Q}^{T}$ current:

$$
\begin{aligned}
b \int_{-\infty}^{\infty} & \left(\left(2 f^{\prime}+A h\right)\left|u^{\prime}\right|^{2}+\left(A h\left(V-\omega^{2}\right)-f V^{\prime}\right)|u|^{2}-\frac{1}{2}\left(f^{\prime \prime \prime}+A h^{\prime \prime}\right)|u|^{2}\right) \\
& \leq-\int_{-\infty}^{\infty}\left(2 f \operatorname{Re}\left(u^{\prime} \bar{H}\right)+\left(f^{\prime}+A h\right) \operatorname{Re}(u \bar{H})+\chi_{1}\left(\omega-\omega_{+} m\right) \operatorname{Im}(H \bar{u})+E \omega \operatorname{Im}(H \bar{u})\right) .
\end{aligned}
$$

Restricting the domain of integration of the left hand side of our estimate then finishes the proof.

8.6. The $\mathcal{G}_{\natural}$ range. This range is manifestly non-superradiant. By the results of Section 6.4, it will follow that, after suitable such choices of $\omega_{\text {high }}$ and $\epsilon_{\text {width }}$, this will be the only range which can contain trapping phenomena; thus, it is only in this range for which we will define a non-zero parameter $r_{\text {trap }}$.

After the final choices of parameters $\omega_{\text {high }}$ and $\epsilon_{\text {width }}$ have been made, then for $(\omega, m, \Lambda) \epsilon$ $\mathcal{G}_{\mathrm{h}}\left(\omega_{\text {high }}, \epsilon_{\text {width }}\right)$, we set the functions $h, \tilde{y}, \hat{y}$ and $\chi_{1}$ appearing in the statement of Theorem 8.1 to be identically 0 . The remaining functions $f$ and $\hat{y}$, the parameter $r_{\text {trap }}$, and the desired coercivity properties are given by the following:

Proposition 8.6.1. Let $a_{0}<M$. Then, for all $\epsilon_{\text {width }}>0$, for all $\omega_{\text {high }}, R_{\infty}$ and E sufficiently big depending on $\epsilon_{\mathrm{width}}$, and for all $0 \leq a \leq a_{0},(\omega, m, \Lambda) \in \mathcal{G}_{\mathrm{h}}\left(\omega_{\mathrm{high}}, \epsilon_{\mathrm{width}}\right)$, there exist functions $f$ and $\hat{y}$ and a value $r_{\text {trap }}$ satisfying the uniform bounds

$$
\begin{gathered}
r_{\text {trap }}=0 \quad \text { or } \quad 0<b<r_{\text {trap }}-r_{+}<B, \\
|f|+\Delta^{-1} r^{2}\left|f^{\prime}\right|+|y| \leq B\left(\epsilon_{\text {width }}\right), \\
f=1, \hat{y}=0 \text { for } r^{*} \geq R_{\infty}^{*},
\end{gathered}
$$

such that, for all smooth solutions $u$ to the radial o.d.e. (44) with right hand side H, satisfying moreover the boundary conditions (51) and (52), we have the estimate

$$
\begin{aligned}
b\left(\epsilon_{\text {width }}\right) \int_{R_{-}^{*}}^{R_{+}^{*}} & \left(\left|u^{\prime}\right|^{2}+\left(\left(\omega^{2}+\Lambda\right)\left(1-r^{-1} r_{\text {trap }}\right)^{2}+1\right)|u|^{2}\right) \\
& \leq \int_{-\infty}^{\infty}\left(-2 f \operatorname{Re}\left(u^{\prime} \bar{H}\right)-f^{\prime} \operatorname{Re}(u \bar{H})+E \omega \operatorname{Em}(H \bar{u})\right)+\int_{-\infty}^{\infty} 2 \hat{y} \operatorname{Re}\left(u^{\prime} \bar{H}\right) .
\end{aligned}
$$

Proof. As noted above, this frequency range, where $\omega^{2}$ is comparable to $\Lambda$, contains the trapping phenomena, but is non-superradiant. For frequencies $(\omega, m, \Lambda) \in \mathcal{G}_{\mathfrak{\natural}}$, Lemma 6.3.1 implies that the potential $V_{0}$ may have at most two critical points. Furthermore, either a maximum $r_{\max }^{0}$ exists or $V_{0}$ is non-increasing on $\left[r_{+}, \infty\right)$; if the maximum exists, then there may also exist a minimum $r_{\min }^{0}$ which will satisfy $r_{\min }^{0}<r_{\max }^{0}$.

In analogy to Lemma 8.3.1 we first must show that for $\omega_{\text {high }}$ sufficiently large, the full potential $V$ enjoys similar properties.

Lemma 8.6.1. For $\epsilon_{\text {width }}$ as above, for all $\omega_{\text {high }}$ sufficiently large depending on $\epsilon_{\text {width }}$ and for $(\omega, m, \Lambda) \in \mathcal{G}_{\mathfrak{\natural}}\left(\omega_{\text {high }}, \epsilon_{\text {width }}\right)$, there exists an $r_{3} \in\left(r_{+}, \infty\right]$ depending on the frequency triple but bounded away from $r_{+}$,

such that for $r \in\left[r_{+}, r_{3}\right]$

$$
r_{3}-r_{+} \geq b\left(\epsilon_{\text {width }}\right)
$$

$$
V(r) \leq \omega^{2}-b\left(\epsilon_{\text {width }}\right) \Lambda .
$$


Furthermore, in the case when $r_{3}<\infty$, then in fact $r_{3} \leq B\left(\omega_{\text {high }}, \epsilon_{\text {width }}\right), r_{\max }^{0}$ exists and the potential $V$ has a unique non-degenerate maximum $r_{\max } \in\left[r_{3}, \infty\right),\left|r_{\max }-r_{\max }^{0}\right| \leq B\left(\epsilon_{\text {width }}\right) \Lambda^{-1}$ and $\frac{d^{2}}{d r^{2}} V\left(r_{\max }\right)<-b\left(\epsilon_{\text {width }}\right) \Lambda$.

Proof. Since $m \omega \notin\left(0, \frac{a m^{2}}{2 M r_{+}}+\alpha \Lambda\right]$ and $\epsilon_{\text {width }} \Lambda \leq \omega^{2} \leq \epsilon_{\mathrm{width}}^{-1} \Lambda$, we find

$$
\omega^{2}-V\left(r_{+}\right)=\omega^{2}-V_{0}\left(r_{+}\right) \geq c \Lambda
$$

where $c=c\left(\epsilon_{\text {width }}\right)$ only depends on the value of $\epsilon_{\text {width }}$. We define $r_{0} \in\left(r_{+}, \infty\right]$ to be the largest value with the property that for all $r \in\left[r_{+}, r_{0}\right)$

$$
V_{0}(r) \leq V_{0}\left(r_{+}\right)+\frac{c}{2} \Lambda \text {. }
$$

If $r_{0}$ is finite then we must have a maximum $r_{\max }^{0}$. Furthermore, $\frac{d V_{0}}{d r}\left(r_{0}\right) \geq 0$; hence, Lemma 6.3.1 implies that if $r_{\min }^{0}$ exists, then

$$
r_{\min }^{0}<r_{0} \leq r_{\max }^{0}
$$

Moreover, Lemma 6.3.1 implies that $r_{\max }^{0}$ is bounded from above by a constant only depending on $\epsilon_{\text {width }}$. On the other hand, since $\left|\frac{d}{d r} V_{0}(r)\right| \leq B\left(\epsilon_{\text {width }}\right) \Lambda r^{-3}$, the value $r_{0}-r_{+}$and thus $r_{\text {max }}^{0}-r_{+}$is bounded from below by a constant only depending on $\epsilon_{\text {width }}$.

We continue to consider the case where $r_{0}<\infty$. Recall from the proof of Lemma 6.3.1 that either $\frac{d}{d r}\left(\left(r^{2}+a^{2}\right)^{3} \frac{d V_{0}}{d r}\right)$ is negative on $\left[r_{+}, \infty\right)$ or there exists a unique value $r_{1} \in\left[r_{+}, r_{\max }^{0}\right)$ such that $\frac{d}{d r}\left(\left(r^{2}+a^{2}\right)^{3} \frac{d V_{0}}{d r}\right)$ is positive on $\left[r_{+}, r_{1}\right)$ and negative on $\left(r_{1}, \infty\right)$. Moreover, since for frequency triples in $\mathcal{G}_{\mathrm{b}}$, the parameter $\sigma=a m \omega / \Lambda$ is bounded by a constant only depending on $\epsilon_{\text {width }}$, the value of $r_{1}$ is uniformly bounded from above by a constant only depending on $\epsilon_{\text {width }}$. We first consider the case where the point $r_{1}$ exists and further split the analysis into two sub-cases based on the value of $V_{0}\left(r_{1}\right)$.

If $V_{0}\left(r_{1}\right) \leq V_{0}\left(r_{+}\right)+\frac{3 c}{4} \Lambda$, then, in view of the fact that $V_{0}$ has a unique maximum at $r_{\max }^{0}$, we have that

$$
V_{0}(r) \leq V_{0}\left(r_{+}\right)+\frac{3 c}{4} \Lambda, \quad \forall r \in\left[r_{+}, \max \left(r_{0}, r_{1}\right)\right] .
$$

Moreover, using that $\left|\frac{d V_{0}}{d r}\right| \leq B\left(\epsilon_{\text {width }}\right) \Lambda r^{-3}$ and that $\Lambda^{-1} \frac{d}{d r}\left(\left(r^{2}+a^{2}\right)^{3} \frac{d}{d r} V_{0}(r)\right)$ is a quadratic polynomial, with coefficients bounded by $\epsilon_{\text {width }}$, vanishing at the unique point $r_{1}$ on the interval $\left[r_{+}, \infty\right)$, we can find a small constant $\delta=\delta\left(\epsilon_{\text {width }}\right)>0$ only depending on $\epsilon_{\text {width }}$ such that

$$
V_{0}(r) \leq V_{0}\left(r_{+}\right)+\frac{3 c}{5} \Lambda, \quad \forall r \in\left[r_{+}, \max \left(r_{0}, r_{1}\right)+\delta\right]
$$

and

$$
\frac{d}{d r}\left(\left(r^{2}+a^{2}\right)^{3} \frac{d}{d r} V_{0}(r)\right)<-c_{1}\left(\epsilon_{\text {width }}\right) \Lambda r^{2}, \quad \forall r \in\left[\max \left(r_{0}, r_{1}\right)+\delta, \infty\right),
$$

where the positive constant $c_{1}$ only depends on $\epsilon_{\text {width }}$.

Now we consider the case where $V_{0}\left(r_{1}\right) \geq V_{0}\left(r_{+}\right)+\frac{3 c}{4} \Lambda$. Then, once again using the bound $\left|\frac{d V_{0}}{d r}\right| \leq B\left(\epsilon_{\text {width }}\right) \Lambda$, we conclude that $r_{1}-r_{0}$ is bounded from below by a small positive constant just depending on $\epsilon_{\text {width }}$. Furthermore, since $\frac{d}{d r} V_{0}\left(r_{0}\right) \geq 0$, we can find a value $r_{0}^{\prime} \in\left[r_{0}, r_{1}\right]$ such that

$$
V_{0}(r) \leq V_{0}\left(r_{+}\right)+\frac{3 c}{4} \Lambda, \quad \forall r \in\left[r_{+}, r_{0}^{\prime}\right]
$$

and

$$
\frac{d}{d r} V_{0}(r) \geq c_{2} \Lambda, \quad \forall r \in\left[r_{0}^{\prime}, r_{1}\right]
$$

where $c_{2}=c_{2}\left(\epsilon_{\text {width }}\right)$ is a positive constant which only depends on $\epsilon_{\text {width }}$.

Moreover, after slightly changing $c_{2}$, the last property can be easily extended to a slightly larger interval

$$
\frac{d}{d r} V_{0}(r) \geq c_{2} \Lambda, \quad \forall r \in\left[r_{0}^{\prime}, r_{1}+\delta\right]
$$


so that $\delta$ only depends on the constant $\epsilon_{\text {width }}$.

$$
\frac{d}{d r}\left(\left(r^{2}+a^{2}\right)^{3} \frac{d}{d r} V_{0}(r)\right)<-c_{3} \Lambda r^{2}, \quad \forall r \in\left[r_{1}+\delta, \infty\right),
$$

for a positive constant $c_{3}=c_{3}\left(\epsilon_{\text {width }}\right)$ which only depends on $\epsilon_{\text {width }}$.

If $r_{1}$ does not exists, the above arguments mutatis mutandis will produce a value $r_{0}^{\prime}$ only depending on the value $\epsilon_{\text {width }}$ such that

$$
\begin{gathered}
V_{0}(r) \leq V_{0}\left(r_{+}\right)+\frac{3 c}{4} \Lambda, \quad \forall r \in\left[r_{+}, r_{0}^{\prime}\right], \\
\frac{d}{d r}\left(\left(r^{2}+a^{2}\right)^{3} \frac{d}{d r} V_{0}(r)\right)<-c_{4}\left(\epsilon_{\text {width }}\right) \Lambda r^{2}, \quad \forall r \in\left[r_{0}^{\prime}, \infty\right),
\end{gathered}
$$

for a positive constant $c_{4}=c_{4}\left(\epsilon_{\text {width }}\right)$ only depending on $\epsilon_{\text {width }}$.

Finally, in both cases $r_{0}<\infty$ and $r_{0}=\infty$ we may therefore claim the existence of a value $r_{3}$ (possibly infinite), bounded away from $r_{+}$by a constant only depending on $\epsilon_{\text {width }}$, such that

$$
V_{0}(r) \leq V_{0}\left(r_{+}\right)+\frac{3 c}{4} \Lambda \leq \omega^{2}-\frac{c}{4} \Lambda, \quad \forall r \in\left[r_{+}, r_{3}\right]
$$

and, such that for any $r \in\left[r_{3}, \infty\right)$, either

$$
\frac{d}{d r} V_{0}(r) \geq b\left(\epsilon_{\text {width }}\right) \Lambda
$$

or

$$
\frac{d}{d r}\left(\left(r^{2}+a^{2}\right)^{3} \frac{d}{d r} V_{0}(r)\right)<-b\left(\epsilon_{\text {width }}\right) \Lambda r^{2} .
$$

We note that if $r_{3}$ is finite, then it is bounded from above by a constant only depending on $\epsilon_{\text {width }}$.

Now, just as we argued in the frequency range $\mathcal{G}^{\sharp}$, adding the bounded potential $V_{1}$, and requiring that $\omega_{\text {high }}$ is sufficiently large finishes the proof.

Before constructing our current, it will be useful to recall that, as observed in Section 8.4. the inequalities $\Lambda \geq|m|(|m|+1)$ and $\Lambda \geq 2 a|m \omega|$ imply that there exists a constant $R_{\mathrm{dec}}^{*} \geq 2 R_{+}^{*}$ only depending on $a_{0}$ and $M$ such that

$$
V^{\prime}<0 \text { for } r^{*} \geq R_{\mathrm{dec}}^{*}
$$

We now construct our current, first under the assumption that $r_{3}^{*}<R_{\mathrm{dec}}^{*}$. Given $E$ sufficiently large depending on $\epsilon_{\text {width }}$, we shall use a combination

$$
\mathrm{Q}=\mathrm{Q}^{f}-4^{y}-E \mathrm{Q}^{T}
$$

of the currents $\mathrm{Q}^{f}, \zeta^{y}$ and $Q^{T}$ where $f, y$ are chosen as described below.

The current $\mathrm{Q}^{f}$ is applied with a function $f$ such that

$$
|f|+\Delta^{-1} r^{2}\left|f^{\prime}\right| \leq B\left(\epsilon_{\text {width }}\right), \quad f\left(r_{+}\right)=0, \quad f^{\prime}>0 \text { for } r \in\left[r_{3}, R_{\infty}\right],
$$

$$
f \text { switches from negative to positive at } r=r_{\max }, \quad f=1 \text { for } r^{*} \geq R_{\mathrm{dec}}^{*} \text {, }
$$

$$
-f V^{\prime}-\frac{1}{2} f^{\prime \prime \prime}(r)>b\left(\epsilon_{\text {width }}\right) \Lambda \frac{\Delta\left(r-r_{\text {max }}\right)^{2}}{r^{7}}, \quad \forall r \in\left[r_{3}, \infty\right) .
$$

In view of the properties of $V$ proven above, such a function can easily be constructed.

The second current will be $\iota^{\hat{y}}$, with

$$
\hat{y}=0 \text { for } r \geq r_{3}, \quad \hat{y}^{\prime}>0 \text { for } r \leq r_{3}, \quad|\hat{y}|+\left|\hat{y}^{\prime}\right| \leq B\left(\epsilon_{\text {width }}\right) .
$$

Such a $\hat{y}$ is now trivial to construct. 
Finally, we subtract the multiple $E \mathrm{Q}^{T}$ of the current $\mathrm{Q}^{T}$. We obtain:

$$
\begin{aligned}
& \int_{-\infty}^{r_{3}}\left(\hat{y}^{\prime}\left(\left|u^{\prime}\right|^{2}+\left(\omega^{2}-V\right)|u|^{2}\right)-\hat{y} V^{\prime}|u|^{2}\right)+\int_{-\infty}^{\infty}\left(2 f^{\prime}\left|u^{\prime}\right|^{2}-\left(f V^{\prime}+\frac{1}{2} f^{\prime \prime \prime}\right)|u|^{2}\right) \\
& +\left(-f\left|u^{\prime}\right|^{2}+\left(\frac{1}{2} E-f\right) \omega^{2}|u|^{2}\right)_{r=\infty}+\left.\left(\frac{1}{2} E \omega\left(\omega-\omega_{+} m\right)-2 \hat{y}\left(\omega-\omega_{+} m\right)^{2}\right)|u|^{2}\right|_{r=r_{+}} \\
& \quad=-\int_{-\infty}^{\infty}\left(2 f \operatorname{Re}\left(u^{\prime} \bar{H}\right)+f^{\prime} \operatorname{Re}(u \bar{H})-E \omega \operatorname{Im}(H \bar{u})\right)+\int_{-\infty}^{r_{3}} 2 \hat{y} \operatorname{Re}\left(u^{\prime} \bar{H}\right) .
\end{aligned}
$$

By the described properties of the potential $V$, the expression $-\left(f V^{\prime}+\frac{1}{2} f^{\prime \prime \prime}\right)$ is positive on the interval $\left[r_{3}, \infty\right)$. On the interval $\left(r_{+}, r_{3}\right]$, we need to choose a function $\hat{y}$ so that in addition to (107) we have

$$
\hat{y}^{\prime}\left(\omega^{2}-V\right)-\hat{y} V^{\prime}-\left(f V^{\prime}+\frac{1}{2} f^{\prime \prime \prime}\right) \geq 0
$$

Since for these values of $r$

$$
\left(\omega^{2}-V\right) \geq b\left(\epsilon_{\text {width }}\right) \Lambda, \quad\left|V^{\prime}\right| \leq B\left(\epsilon_{\text {width }}\right) \Lambda \frac{\Delta}{r^{2}}, \quad|f|+\left|f^{\prime \prime \prime}\right| \leq B\left(\epsilon_{\text {width }}\right) \frac{\Delta}{r^{2}},
$$

it suffices to fulfill the inequality

$$
\frac{d}{d r} \hat{y} \geq-\hat{y} C+C
$$

provided that $C$ is sufficiently large only depending on $\epsilon_{\text {width }}$. The function

$$
\hat{y}=1-e^{C\left(r_{3}-r\right)}
$$

satisfies all the above criteria. Note that the constant $C$ only depends on $\epsilon_{\text {width. }}$. Finally, for all $E$ such that $C \ll E$, the non-superradiant condition $m \omega \notin\left(0, m \omega_{+}\right]$and the boundary condition $u^{\prime}=i \omega u$ at $r=\infty$ ensure that both boundary terms at $r=r_{+}$and $r=\infty$ are positive. After restricting the domain of integration of the left hand side of our estimate, we have obtained (102), defining

$$
r_{\text {trap }}=r_{\max } .
$$

In the case $\infty \geq r_{3} \geq R_{\mathrm{dec}}^{*}$ we construct our current as follows. As above we will have

$$
\mathrm{Q}=\mathrm{Q}^{f}+r^{\hat{y}}-E \mathrm{Q}^{T} \text {. }
$$

We define

$$
\begin{gathered}
\hat{y}=1-e^{\hat{C}\left(R_{\mathrm{dec}}+2-r\right)} \text { for } r \leq R_{\mathrm{dec}}+2, \\
\hat{y}=0 \text { for } r \geq R_{\mathrm{dec}}+2 .
\end{gathered}
$$

Note we shall satisfy (109) with $C$ replaced by $\hat{C}$. Thus, arguing just as in the case when $r_{3}<R_{\mathrm{dec}}$, for a sufficiently large $\hat{C}$ we will have

$$
\int_{-\infty}^{R_{\mathrm{dec}+1}}\left(\hat{y}^{\prime}\left(\left|u^{\prime}\right|^{2}+\left(\omega^{2}-V\right)|u|^{2}\right)-\hat{y} V^{\prime}|u|^{2}\right) \geq b\left(\epsilon_{\mathrm{width}}\right) \int_{R_{-}^{*}}^{R_{\mathrm{dec}+1}}\left(\left|u^{\prime}\right|^{2}+\omega^{2}|u|^{2}\right) .
$$

Next, we let $f$ be any smooth function such that

$$
f^{\prime} \geq 0, \quad f=0 \text { for } r \in\left[r_{+}, R_{\mathrm{dec}}\right], \quad f=1 \text { for }\left[R_{\mathrm{dec}}+1, \infty\right), \quad|f|+\left|f^{\prime}\right|+\left|f^{\prime \prime \prime}\right| \leq B .
$$

Such an $f$ is trivial to construct.

Requiring $\omega_{\text {high }}$ to be sufficiently large depending on $\epsilon_{\text {width }}$, we shall have

$$
\begin{gathered}
\int_{R_{\mathrm{dec}}}^{\infty}\left(\hat{y}^{\prime}\left(\left|u^{\prime}\right|^{2}+\left(\omega^{2}-V\right)|u|^{2}\right)-\hat{y} V^{\prime}|u|^{2}\right)+\int_{R_{\mathrm{dec}}}^{\infty}\left(2 f^{\prime}\left|u^{\prime}\right|^{2}-\left(f V^{\prime}+\frac{1}{2} f^{\prime \prime \prime}\right)|u|^{2}\right) \\
\geq \int_{R_{\mathrm{dec}}^{*}}^{R_{\mathrm{dec}}^{*}+1}\left(b \omega_{\text {high }}^{2}-\frac{1}{2} f^{\prime \prime \prime}\right)|u|^{2} \geq 0 .
\end{gathered}
$$

Thus, the bulk term of the estimate corresponding to Q is positive. Just as in the case $r_{3}<R_{\text {dec }}$, requiring that $E$ is large enough depending on $\epsilon_{\text {width }}$ will guarantee that the boundary terms are controlled. Finally, we require that $R_{\infty}^{*} \geq R_{\mathrm{dec}}^{*}+1$. This gives again (102) defining $r_{\text {trap }}=0$. 
8.7. The $\mathcal{G}_{b}$ range. This range again depends on $\omega_{\text {high }}$, and $\epsilon_{\text {width. As opposed to the Propositions }}$ concerning the other ranges which restrict the choices of one or both these parameters, in the range $\mathcal{G}_{b}\left(\omega_{\text {high }}, \epsilon_{\text {width }}\right)$, estimates can be obtained for arbitrary $\omega_{\text {high }}>0$ and $\epsilon_{\text {width }}>0$, but the relevant constants will degenerate as $\omega_{\text {high }} \rightarrow \infty, \epsilon_{\text {width }} \rightarrow 0$.

We shall split the frequency range $\mathcal{G}_{b}$ into four subcases, considering each separately. We will see the above degeneration in the last of the cases. We note that our decomposition will not however distinguish between superradiant and non-superradiant frequencies. It should be clear to the reader how the constructions could be simplified if restricted to the non-superradiant case.

The split will rely on the introduction of a further small parameter $\tilde{a}_{0}$. This parameter is for now free-we choose it in Section 8.8

8.7.1. The subrange $|\omega| \leq \omega_{\text {low }}, 0 \leq a<\tilde{a}_{0}$ and $m \neq 0$. Given the final choice of parameters, $\omega_{\text {high }}$, $\epsilon_{\text {width }}$ and $\omega_{\text {low }}$, then for $(\omega, m, \Lambda) \in \mathcal{G}_{b}\left(\omega_{\text {high }}, \epsilon_{\text {width }}\right)$ such that $|\omega| \leq \omega_{\text {low }}$ and $a<\tilde{a}_{0}$, we will set the functions $f$ and $\tilde{y}$ together with the parameter $r_{\text {trap }}$ to be 0 . The remaining functions $y, \hat{y}, h, \chi_{1}$, $\chi_{2}$ and the desired coercivity properties are given by the following

Proposition 8.7.1. Let $a_{0}<M$. Then, for all $\omega_{\text {high }}>0$, $\epsilon_{\text {width }}>0$, for all $\omega_{\text {low }}>0, \tilde{a}_{0}>0$ sufficiently small depending on $\omega_{\text {high }}$ and $\epsilon_{\text {width }}$, for all $R_{\infty}$ sufficiently large, for all $E \geq 2,0 \leq a \leq a_{0}$, and for all $(\omega, m, \Lambda) \in \mathcal{G}_{b}\left(\omega_{\text {high }}, \epsilon_{\text {width }}\right)$ such that $|\omega| \leq \omega_{\text {low }}$ and $0 \leq a<\tilde{a}_{0}$, there exist functions $y$, $\hat{y}, \chi_{1}, \chi_{2}$ and $h$, satisfying the uniform bounds

$$
\begin{gathered}
|y|+|\hat{y}|+|h|+\left|\chi_{2}\right| \leq B, \\
\chi_{2}=1, \chi_{1}=0, y=1, \hat{y}=0, h=0 \text { for } r^{*} \geq R_{\infty}^{*},
\end{gathered}
$$

such that, for all smooth solutions $u$ to the radial o.d.e. (44) with right hand side $H$, satisfying moreover the boundary conditions (51) and (52), we have the estimate

$b \int_{R_{-}^{*}}^{R_{+}^{*}}\left(\left|u^{\prime}\right|^{2}+|u|^{2}\right) \leq \int_{-\infty}^{\infty}\left(2(y+\hat{y}) \operatorname{Re}\left(u^{\prime} \bar{H}\right)+h \operatorname{Re}(u \bar{H})+E \omega \chi_{1} \operatorname{Im}(H \bar{u})+\chi_{2}\left(\omega-\omega_{+} m\right) \operatorname{Im}(H \bar{u})\right)$.

Proof. The construction of our current is inspired by the treatment of similar frequency regimes in [6] and [41].

The following three properties are easily verified:

(1) For every $-\infty<\alpha<\beta<\infty$, if we require $\tilde{a}$ and $\omega_{\text {low }}$ sufficiently small, both depending on $\alpha$ and $\beta$, then $r \in[\alpha, \beta] \Rightarrow V-\omega^{2}>0$.

(2) For sufficiently large $r^{*}$, independent of the frequency parameters, we have $V^{\prime}<0$.

(3) For sufficiently small $\tilde{a}_{0}$ and sufficiently negative $r^{*}$, independent of the frequency parameters, we have $V^{\prime}>0$.

Let's introduce the set of relevant constants.

(1) Requiring that $\tilde{a}_{0}$ is sufficiently small, let $R_{1}^{*}<R_{-}^{*}$ be a fixed negative constant chosen so that $r^{*} \leq R_{1}^{*}$ implies that $V^{\prime}>0$ and $\left(r^{*}\left(V-\left.V\right|_{r=r_{+}}\right)\right)^{\prime}>0$.

(2) Let $R_{2}^{*}>R_{+}^{*}$ be a fixed positive constant chosen so that $r^{*} \geq R_{2}^{*}$ implies $V^{\prime}<0$ and $\left(r^{*} V\right)^{\prime}<0$.

(3) Let $\epsilon>0$ be a sufficiently small positive constant to be fixed later.

(4) Let $p=p(\epsilon)>0$ be a sufficiently small positive constant depending on $\epsilon$.

We now construct our current $\mathrm{Q}$ in a step by step fashion. Choose a function $h$ satisfying

$$
\begin{gathered}
h=1 \text { for } r^{*} \in\left[R_{1}^{*}, R_{2}^{*}\right], \quad h=0 \text { for } r^{*} \in\left(-\infty, e^{p^{-1}} R_{1}^{*}\right], \quad h \geq 0, \\
h=0 \text { for } r^{*} \in\left[e^{p^{-1}} R_{2}^{*}, \infty\right), \quad\left|h^{\prime \prime}\right| \leq \frac{B p}{\left|r^{*}\right|^{2}} \text { when } r^{*} \in\left[e^{p^{-1}} R_{1}^{*}, R_{1}^{*}\right] \cup\left[R_{2}^{*}, e^{p^{-1}} R_{2}^{*}\right] .
\end{gathered}
$$

Note that one may easily construct a function $h$ satisfying (113) and (114). 
We then apply a $\mathrm{Q}^{h}$ current:

$$
\int_{-\infty}^{\infty}\left(h\left|u^{\prime}\right|^{2}+\left(h\left(V-\omega^{2}\right)-\frac{1}{2} h^{\prime \prime}\right)|u|^{2}\right)=-\int_{-\infty}^{\infty} h \operatorname{Re}(u \bar{H}) .
$$

The integrand of the left hand side of the estimate (115) will cease to be non-negative for $r^{*} \epsilon$ $\left[e^{p^{-1}} R_{1}^{*}, R_{1}^{*}\right] \cup\left[R_{2}^{*}, e^{p^{-1}} R_{2}^{*}\right]$. We will produce a non-negative integrand by adding in $\zeta^{y}$ and $\zeta^{\hat{y}}$ currents.

Define a function $y$ by

$$
\begin{aligned}
& y=0 \text { for } r^{*} \in\left(-\infty, R_{2}^{*}-1\right), \quad y=\frac{r^{*}-R_{2}^{*}+1}{2} \text { for } r^{*} \in\left[R_{2}^{*}-1, R_{2}^{*}\right), \\
& y=\epsilon\left(\frac{1}{r^{*} V}-\frac{1}{\left.R_{2}^{*} V\right|_{r^{*}=R_{2}^{*}}}\right)+\frac{1}{2} \text { for } r^{*} \in\left[R_{2}^{*}, e^{p^{-1}} R_{2}^{*}\right],
\end{aligned}
$$

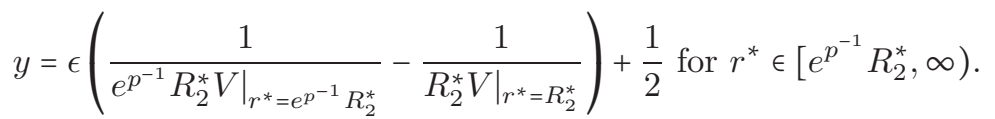

Note that we have chosen $R_{2}^{*}$ so that we will have $y^{\prime} \geq 0$. Of course, we also have $y \geq 0$.

Now we add in a $\zeta^{y}$ current to (115) and obtain

$$
\begin{gathered}
\int_{-\infty}^{\infty}\left(\left(h+y^{\prime}\right)\left|u^{\prime}\right|^{2}+\left(y^{\prime} \omega^{2}+h\left(V-\omega^{2}\right)-(y V)^{\prime}-\frac{1}{2} h^{\prime \prime}\right)|u|^{2}\right)= \\
y(\infty)\left(\left|u^{\prime}\right|^{2}+\omega^{2}|u|^{2}\right)_{r=\infty}-\int_{-\infty}^{\infty}\left(2 y \operatorname{Re}\left(u^{\prime} \bar{H}\right)+h \operatorname{Re}(u \bar{H})\right) .
\end{gathered}
$$

We will now show that if we require $\omega_{\text {low }}$ and $\tilde{a}_{0}$ to be sufficiently small depending on appropriate choices of $\epsilon$ and $p$, the integrand of the left hand side of (119) is non-negative in the region $r^{*} \epsilon$ $\left[R_{+}^{*}, \infty\right)$. Since $h, y^{\prime} \geq 0$ it suffices to show that the term $h\left(V-\omega^{2}\right)-(y V)^{\prime}-\frac{1}{2} h^{\prime \prime}$ is non-negative.

The function $y$ vanishes and $h=1$ in the region $r^{*} \in\left[R_{+}^{*}, R_{2}^{*}-1\right)$, and thus we have

$$
r^{*} \in\left[R_{+}^{*}, R_{2}^{*}-1\right) \Rightarrow h\left(V-\omega^{2}\right)-(y V)^{\prime}-\frac{1}{2} h^{\prime \prime}=V-\omega^{2} .
$$

If $\omega_{\text {low }}$ and $\tilde{a}_{0}$ are sufficiently small, then $V-\omega^{2}$ will be positive in this region.

Next, we have

$$
r^{*} \in\left[R_{2}^{*}-1, R_{2}^{*}\right) \Rightarrow h\left(V-\omega^{2}\right)-(y V)^{\prime}-\frac{1}{2} h^{\prime \prime}=V-\omega^{2}-\frac{1}{2} V-y V^{\prime} .
$$

Recall that we chose $R_{2}^{*}$ so that $V^{\prime}<0$ in this region. Since $y>0$, we then get

$$
r^{*} \in\left[R_{2}^{*}-1, R_{2}^{*}\right) \Rightarrow V-\omega^{2}-\frac{1}{2} V-y V^{\prime} \geq \frac{1}{2} V-\omega^{2} .
$$

Now, it is clear that if $\omega_{\text {low }}$ and $\tilde{a}_{0}$ are sufficiently small, then $\frac{1}{2} V-\omega^{2}$ will be positive in this region.

Next we consider the region $r^{*} \in\left[R_{2}^{*}, e^{p^{-1}} R_{2}^{*}\right)$. As usual, we start by noting that if we require $\omega_{\text {low }}$ and $\tilde{a}_{0}$ to be sufficiently small depending on $p$, then $V-\omega^{2}>0$ in the region $r^{*} \in\left[R_{2}^{*}, e^{p^{-1}} R_{2}^{*}\right)$. Hence, we will have

$$
r^{*} \in\left[R_{2}^{*}, e^{p^{-1}} R_{2}^{*}\right) \Rightarrow h\left(V-\omega^{2}\right)-(y V)^{\prime}-\frac{1}{2} h^{\prime \prime} \geq \frac{\epsilon-B p}{\left(r^{*}\right)^{2}}-\left(\frac{1}{2}-\frac{\epsilon}{\left.R_{2}^{*} V\right|_{r^{*}=R_{2}^{*}}}\right) V^{\prime} .
$$

Again, we recall that $V^{\prime}<0$ for $r^{*}>R_{5}^{*}$. Furthermore, as long as we require $\epsilon$ to be sufficiently small, we will have $\frac{1}{2}-\frac{\epsilon}{\left.R_{2}^{*} V\right|_{r^{*}=R_{2}^{*}}}>0$. Finally, we may choose $p$ small enough depending on $\epsilon$ so that the first term on the right hand side of (120) is also positive.

In the region $r^{*} \in\left[e^{p^{-1}} R_{2}^{*}, \infty\right)$ we have that $h=0$ and $y$ is constant. Since $V^{\prime}<0$ in this region, we have

$$
h\left(V-\omega^{2}\right)-(y V)^{\prime}-\frac{1}{2} h^{\prime \prime}=-y(\infty) V^{\prime}>0 .
$$


Thus as long as $\epsilon>0$ is sufficiently small, $p$ is sufficiently small depending on $\epsilon$ and $\omega_{\text {low }}$ and $\tilde{a}_{0}$ are sufficiently small depending on $p$, the integrand of the left hand side of (119) is non-negative for $r^{*} \geq R_{2}^{*}$; however, it is still not non-negative for $r^{*}<R_{1}^{*}$. To remedy this we will employ a $4^{\hat{y}}$ current with a function $\hat{y}$ whose properties as $r^{*} \rightarrow-\infty$ will mimic the properties of $y$ as $r^{*} \rightarrow \infty$. The key point which allows us to carry out an analogous construction is that $V^{\prime}>0$ for $r^{*}$ sufficiently close to $-\infty$.

We define

$$
\begin{gathered}
\hat{y}=0 \text { for } r^{*} \in\left(R_{1}^{*}+1, \infty\right), \quad \hat{y}=\frac{r^{*}-R_{1}^{*}-1}{2} \text { for } r^{*} \in\left[R_{1}^{*}, R_{1}^{*}+1\right), \\
\hat{y}=\epsilon\left(\frac{1}{r^{*} \tilde{V}}-\frac{1}{\left.R_{1}^{*} \tilde{V}\right|_{r^{*}=R_{1}^{*}}}\right)-\frac{1}{2} \text { for } r^{*} \in\left[e^{p^{-1}} R_{1}^{*}, R_{1}^{*}\right), \\
\hat{y}=\epsilon\left(\frac{1}{\left.e^{p^{-1}} R_{1}^{*} \tilde{V}\right|_{r^{*}=e^{p^{-1}} R_{1}^{*}}}-\frac{1}{\left.R_{1}^{*} \tilde{V}\right|_{r^{*}=R_{1}^{*}}}\right)-\frac{1}{2} \text { for } r^{*} \in\left(-\infty, e^{p^{-1}} R_{1}^{*}\right] .
\end{gathered}
$$

Here $\tilde{V} \doteq V-\left.V\right|_{r=r_{+}}$. Note that we have chosen $R_{1}^{*}$ so that $\hat{y}^{\prime} \geq 0$. Of course, we also have $\hat{y} \leq 0$.

Now we add a $\zeta^{\hat{y}}$ current to (119). We obtain

$$
\begin{gathered}
\int_{-\infty}^{\infty}\left(\left(h+y^{\prime}+\hat{y}^{\prime}\right)\left|u^{\prime}\right|^{2}+\left(y^{\prime} \omega^{2}+\hat{y}^{\prime}\left(\omega-\omega_{+} m\right)^{2}+h\left(V-\omega^{2}\right)-(y V)^{\prime}-(\hat{y} \tilde{V})^{\prime}-\frac{1}{2} h^{\prime \prime}\right)|u|^{2}\right)= \\
y(\infty)\left(\left|u^{\prime}\right|^{2}+\omega^{2}|u|^{2}\right)_{r=\infty}+|\hat{y}(-\infty)|\left(\left|u^{\prime}\right|^{2}+\left(\omega-\omega_{+} m\right)^{2}|u|^{2}\right)_{r=r_{+}}-\int_{-\infty}^{\infty}\left((y+\hat{y}) \operatorname{Re}\left(u^{\prime} \bar{H}\right)+h \operatorname{Re}(u \bar{H})\right) .
\end{gathered}
$$

Now, keeping in mind that $V^{\prime}>0$ for sufficiently negative $r^{*}$ and repeating the argument, mutatis mutandis, which showed that $r^{*} \geq R_{2}^{*} \Rightarrow h\left(V-\omega^{2}\right)-(y V)^{\prime}-\frac{1}{2} h^{\prime \prime} \geq 0$ we obtain that

$$
r^{*} \leq R_{1}^{*} \Rightarrow h\left(V-\omega^{2}\right)-(\hat{y} \tilde{V})^{\prime}-\frac{1}{2} h^{\prime \prime} \geq 0 .
$$

We conclude that the integrand of the left hand side of (124) is non-negative and is greater than

$$
b \int_{R_{-}^{*}}^{R_{+}^{*}}\left(\left|u^{\prime}\right|^{2}+|u|^{2}\right) \text {. }
$$

We may now fix the constants $\epsilon$ and $p$.

It remains to absorb the boundary terms on the right hand side of (124). We start with the term at $r=\infty$. Let $\chi_{2}$ be a function which is identically 1 for $r^{*} \geq R_{+}^{*}$ and identically 0 for $r^{*} \leq R_{-}^{*}$. Requiring that $E \geq 2$, we obtain

$$
\begin{aligned}
y(\infty)\left(\left|u^{\prime}\right|^{2}+\omega^{2}|u|^{2}\right)_{r=\infty} & \leq E y(\infty) \int_{-\infty}^{\infty}\left(\chi_{2} \mathrm{Q}^{T}\right)^{\prime} \\
& \leq B\left(\omega_{\text {high }}, \epsilon_{\text {width }}\right) \omega_{\text {low }} \int_{R_{-}^{*}}^{R_{+}^{*}}\left(\left|u^{\prime}\right|^{2}+|u|^{2}\right)+E y(\infty) \omega \int_{-\infty}^{\infty} \chi_{2} \operatorname{Im}(H \bar{u}) .
\end{aligned}
$$

Taking $\omega_{\text {low }}$ sufficiently small, we may add this in to our previous estimate and obtain

$$
\begin{aligned}
& b \int_{R_{-}^{*}}^{R_{+}^{*}}\left(\left|u^{\prime}\right|^{2}+|u|^{2}\right) \leq \\
& \quad B\left(\left|u^{\prime}\right|^{2}+\left(\omega-\omega_{+} m\right)^{2}|u|^{2}\right)_{r=r_{+}}-\int_{-\infty}^{\infty}\left((y+\hat{y}) \operatorname{Re}\left(u^{\prime} \bar{H}\right)+h \operatorname{Re}(u \bar{H})+E y(\infty) \omega \chi_{2} \operatorname{Im}(H \bar{u})\right) .
\end{aligned}
$$


Let $\chi_{1}$ be a function which is identically 1 for $r^{*} \in\left(-\infty, R_{1}^{*}\right)$ and identically 0 for $r^{*} \geq R_{2}^{*}$. We obtain

$$
\begin{aligned}
B\left(\left|u^{\prime}\right|^{2}+\left(\omega-\omega_{+} m\right)^{2}|u|^{2}\right)_{r=r_{+}} & =B \int_{-\infty}^{\infty}\left(\chi_{1} \mathrm{Q}^{K}\right)^{\prime} \\
& \leq B\left(\omega_{\text {high }}, \epsilon_{\text {width }}\right)\left(\omega_{\text {low }}+\tilde{a}_{0}\right) \int_{R_{-}^{*}}^{R_{+}^{*}}\left(\left|u^{\prime}\right|^{2}+|u|^{2}\right)+B\left(\omega-\omega_{+} m\right) \int_{-\infty}^{\infty} \chi_{1} \operatorname{Im}(H \bar{u}) .
\end{aligned}
$$

Thus, it is clear that if require that $\omega_{\text {low }}$ and $\tilde{a}_{0}$ are sufficiently small, depending on $\omega_{\text {high }}$ and $\epsilon_{\text {width }}$, we may multiply $\chi_{1}$ by a bounded constant, add in $\chi_{1} \mathrm{Q}^{K}$ to our current and obtain

$b \int_{R_{-}^{*}}^{R_{+}^{*}}\left(\left|u^{\prime}\right|^{2}+|u|^{2}\right) \leq-\int_{-\infty}^{\infty}\left((y+\hat{y}) \operatorname{Re}\left(u^{\prime} \bar{H}\right)+h \operatorname{Re}(u \bar{H})+E y(\infty) \omega \chi_{2} \operatorname{Im}(H \bar{u})+\left(\omega-\omega{ }_{+} m\right) \chi_{1} \operatorname{Im}(H \bar{u})\right)$.

Finally, we may rescale all of the multipliers so that $y(\infty)=1$. We obtain

$$
b \int_{R_{-}^{*}}^{R_{+}^{*}}\left(\left|u^{\prime}\right|^{2}+|u|^{2}\right) \leq-\int_{-\infty}^{\infty}\left((y+\hat{y}) \operatorname{Re}\left(u^{\prime} \bar{H}\right)+h \operatorname{Re}(u \bar{H})+E \chi_{2} \operatorname{Im}(H \bar{u})+\left(\omega-\omega_{+} m\right) \chi_{1} \operatorname{Im}(H \bar{u})\right) .
$$

Of course, $R_{\infty}^{*}$ is simply required to be larger than $e^{p^{-1}} R_{2}^{*}$.

Remark 8.7.1. The above proof does not use the assumption $m \neq 0$. We only include $m \neq 0$ in the definition of the frequency range so that the set of frequencies covered by Proposition 8.7.1 is disjoint from the set of frequencies covered by Proposition 8.7.2.

8.7.2. The subrange $|\omega| \leq \omega_{\text {low }}$ and $m=0$. Given the final choice of parameters, $\omega_{\text {high }}, \epsilon_{\text {width }}$ and $\omega_{\text {low }}$, then for $(\omega, m, \Lambda) \in \mathcal{G}_{b}\left(\omega_{\text {hig }}, \epsilon_{\text {width }}\right)$ such that $|\omega| \leq \omega_{\text {low }}$ and $m=0$, we will set the functions $f$, $\tilde{y}$ and $\chi_{1}$ together with the parameter $r_{\text {trap }}$ to be 0 . The remaining functions $y, \hat{y}, h$ and the desired coercivity properties are given by the following

Proposition 8.7.2. Let $a_{0}<M$. Then, for all $\omega_{\text {high }}>0$, $\epsilon_{\text {width }}>0$, for all $\omega_{\text {low }}>0$ sufficiently small depending on $\omega_{\text {high }}$ and $\epsilon_{\text {width }}$, for all $R_{\infty}$ sufficiently large, for all $E \geq 2,0 \leq a \leq a_{0}$, and for all $(\omega, m, \Lambda) \in \mathcal{G}_{b}\left(\omega_{\text {high }}, \epsilon_{\text {width }}\right)$ such that $|\omega| \leq \omega_{\text {low }}$ and $m=0$, there exists functions $y, \hat{y}$ and $h$, satisfying the uniform bounds

$$
\begin{gathered}
|y|+|\hat{y}|+|h| \leq B, \\
y=1, h=0, \hat{y}=0 \text { for } r^{*} \geq R_{\infty}^{*},
\end{gathered}
$$

such that, for all smooth solutions $u$ to the radial o.d.e. (44) with right hand side $H$, satisfying moreover the boundary conditions (51) and (52), we have the estimate

$$
b \int_{R_{-}^{*}}^{R_{+}^{*}}\left(\left|u^{\prime}\right|^{2}+|u|^{2}\right) \leq-\int_{-\infty}^{\infty}\left(2(y+\hat{y}) \operatorname{Re}\left(u^{\prime} \bar{H}\right)+h \operatorname{Re}(u \bar{H})+\operatorname{E\omega } \operatorname{Im}(H \bar{u})\right) .
$$

Proof. Observe that the properties of the potential $V$ used in the proof of Proposition 8.7 .1 also hold here:

(1) For every $-\infty<\alpha<\beta<\infty$, if we require $\omega_{\text {low }}$ sufficiently small, both depending on $\alpha$ and $\beta$, then $r \in[\alpha, \beta] \Rightarrow V-\omega^{2}>0$.

(2) For sufficiently large $r^{*}$, independent of the frequency parameters, we have $V^{\prime}<0$.

(3) For sufficiently negative $r^{*}$, independent of the frequency parameters, we have $V^{\prime}>0$.

Using these observations, one may repeat, mutatis mutandis, the current construction from the proof of Proposition 8.7.1 In fact, the situation is strictly better here; since this proposition concerns a non-superradiant regime, we may set $\chi_{1}=0$. One obtains

$$
b \int_{R_{-}^{*}}^{R_{+}^{*}}\left(\left|u^{\prime}\right|^{2}+|u|^{2}\right) \leq-\int_{-\infty}^{\infty}\left(2(y+\hat{y}) \operatorname{Re}\left(u^{\prime} \bar{H}\right)+h \operatorname{Re}(u \bar{H})+E \operatorname{Im}(H \bar{u})\right) .
$$


8.7.3. The subrange $|\omega| \leq \omega_{\text {low }}, m \neq 0$ and $a \geq \tilde{a}_{0}$ (the near stationary subcase). Although these frequencies are near-stationary, we will here be able to effectively exploit the non-vanishing of $a$ and the bound $|m| \geq 1$.

For $(\omega, m, \Lambda) \in \mathcal{G}_{b}, m \neq 0$, and $a \geq \tilde{a}_{0}$, we set the functions $f$ and $\hat{y}$ together with the parameter $r_{\text {trap }}$ to 0 . The remaining functions $\tilde{y}, y, h, \chi_{1}$ and $\chi_{2}$ and the desired coercivity properties are given by the following:

Proposition 8.7.3. Let $a_{0}<M$. Then, for all $\omega_{\text {high }}>0$, $\epsilon_{\text {width }}>0, E \geq 2$, for all $\omega_{\text {low }}>0$ sufficiently small depending on $\tilde{a}_{0}$ and $E$, for all $R_{\infty}$ sufficiently large depending on $\tilde{a}_{0}, 0 \leq a \leq a_{0}$, and for all $(\omega, m, \Lambda) \in \mathcal{G}_{\mathrm{b}}\left(\omega_{\mathrm{high}}, \epsilon_{\mathrm{width}}\right)$ such that $|\omega| \leq \omega_{\text {low }}, m \neq 0$ and $a \geq \tilde{a}_{0}$, there exist functions $\tilde{y}, y, h, \chi_{1}$ and $\chi_{2}$, satisfying the uniform bounds

$$
\begin{gathered}
|\tilde{y}|+|y|+|h|+\left|\chi_{1}\right|+\left|\chi_{2}\right| \leq B\left(\tilde{a}_{0}\right), \\
|\tilde{y}| \leq B \exp (-b r), y=1, h=0 \text { for } r^{*} \geq R_{\infty}^{*},
\end{gathered}
$$

such that, for all smooth solutions $u$ to the radial o.d.e. (44) with right hand side $H$, satisfying moreover the boundary conditions (51) and (52), we have the estimate

$$
\begin{aligned}
b\left(\tilde{a}_{0}\right) & \int_{R_{-}^{*}}^{R_{+}^{*}}\left(\left|u^{\prime}\right|^{2}+|u|^{2}\right) \\
& \leq \int_{-\infty}^{\infty}\left(-2 \tilde{y} \operatorname{Re}\left(u^{\prime} \bar{H}\right)-E \chi_{2} \omega \operatorname{Im}(H \bar{u})-2 \chi_{1}\left(\omega-\omega_{+} m\right) \operatorname{Im}(H \bar{u})-h \operatorname{Re}(u \bar{H})-2 y \operatorname{Re}\left(u^{\prime} \bar{H}\right)\right) .
\end{aligned}
$$

Proof. Our current will be of the form

$$
\mathrm{Q}=\zeta^{\tilde{y}}+\mathrm{Q}^{h}+\zeta^{y}-\chi_{1} \mathrm{Q}^{K}-E \chi_{2} \mathrm{Q}^{T},
$$

for suitable functions $\tilde{y}, h, y, \chi_{1}$ and $\chi_{2}$.

As we did for the frequency range $\mathcal{G} \sharp$, for the purposes of exposition we shall construct the current in a step by step fashion. The first important observation is that the assumptions $m \neq 0$ and $a \geq \tilde{a}_{0}$ imply that $\omega_{0}^{2}:=\left(\omega-\omega_{+} m\right)^{2} \geq b\left(\tilde{a}_{0}\right)$ as long as $\omega_{\text {low }} \ll \tilde{a}_{0}$. The second important observation is that $V=\frac{\Lambda}{r^{2}}+O\left(r^{-3}\right)$ as $r \rightarrow \infty$ and, since $m \neq 0, \Lambda \geq 2$. This implies that for any $1 \ll \alpha \ll \beta<\infty$, then requiring that $\omega_{\text {low }}$ is small enough, depending on $\alpha$ and $\beta$, we have

$$
r \in[\alpha, \beta] \Rightarrow V-\omega^{2} \geq \frac{b}{r^{2}}
$$

in this frequency range. We shall exploit this positivity via the use of a $\mathrm{Q}^{h}$ current.

Let us now introduce the set of relevant constants. Let $p>0, R_{1}<R_{2}<R_{3}<e^{p^{-1}} R_{3}, C>0$ and $c>0$ be constants such that

(1) $C=C\left(\tilde{a}_{0}\right)$ is sufficiently large.

(2) $c$ is sufficiently small.

(3) $R_{1}$ is sufficiently large.

(4) $c \omega_{0}^{-2} R_{1} \exp \left(B C R_{1}\right) \ll R_{2}$.

(5) $R_{2} \ll R_{3}$.

(6) $p \ll R_{3}^{-3}$.

(7) $\omega_{\text {low }}^{2} \ll c E^{-1} \omega_{0}^{2} \exp \left(-p^{-1}\right) \exp \left(-B C R_{2}\right) R_{3}^{-4}$ and, requiring $\omega_{\text {low }}$ sufficiently small, $r \in\left[R_{1}, e^{p^{-1}} R_{3}\right] \Rightarrow$ $V-\omega^{2} \geq b r^{-2}$.

We write

$$
\omega^{2}-V=: \omega_{0}^{2}-\tilde{V}
$$

where $\tilde{V}\left(r_{+}\right)=0$. Let $v(r)$ be a positive function such that

$$
v=\Delta \text { near } r_{+}, \quad v=1 \text { when } r^{*} \geq R_{\infty}^{*}, \quad|v| \leq B .
$$


Then we define

$$
\tilde{y}\left(r^{*}\right):=-\exp \left(-C \int_{-\infty}^{r^{*}} v d r^{*}\right)
$$

and consider the current $4^{\tilde{y}}$. Note that $\tilde{y}(-\infty)=-1$ and $\tilde{y}(\infty)=0$. We obtain

$$
\int_{-\infty}^{\infty}\left(\tilde{y}^{\prime}\left|u^{\prime}\right|^{2}+\left(\tilde{y}^{\prime} \omega_{0}^{2}-(\tilde{y} \tilde{V})^{\prime}\right)|u|^{2}\right)=\left(\left|u^{\prime}\right|^{2}+\left(\omega-\omega_{+} m\right)^{2}|u|^{2}\right)_{r=r_{+}}-\int_{-\infty}^{\infty}\left(2 \tilde{y} \operatorname{Re}\left(u^{\prime} \bar{H}\right)\right) .
$$

Remark 8.7.2. Note that unlike every other microlocal current we have considered, this $\tilde{y}$ cannot be taken independent of the frequency parameters when $r^{*} \geq R_{\infty}^{*}$ (since this is the only regime which employs a $\zeta^{\tilde{y}}$ current where the seed function $\tilde{y}$ is negative for large $r$ ). Nevertheless, the exponential decay of $\tilde{y}$ as $r \rightarrow \infty$ will allow us to handle this when we re-sum (see Section Q).

We now turn to the $(\tilde{y} \tilde{V})^{\prime}|u|^{2}$ term on the left hand side of (135) which threatens to destroy our estimate:

$$
\begin{aligned}
& \left.\left.\left|\int_{-\infty}^{\infty}(\tilde{y} \tilde{V})^{\prime}\right| u\right|^{2}|=2| \int_{-\infty}^{\infty} 2 \tilde{y} \tilde{V} \operatorname{Re}\left(u^{\prime} \bar{u}\right)\left|\leq \epsilon \int_{-\infty}^{\infty} \tilde{y}^{\prime}\right| u^{\prime}\right|^{2}+B \epsilon^{-1} \int_{-\infty}^{\infty} \tilde{y}^{\prime} \omega_{0}^{2} \frac{\tilde{y}^{2} \tilde{V}^{2}}{\left(\tilde{y}^{\prime}\right)^{2} \omega_{0}^{2}}|u|^{2}= \\
& \epsilon \int_{-\infty}^{\infty} \tilde{y}^{\prime}\left|u^{\prime}\right|^{2}+B \epsilon^{-1} \int_{-\infty}^{\infty} \tilde{y}^{\prime} \omega_{0}^{2} \frac{\tilde{V}^{2}}{C^{2} v^{2} \omega_{0}^{2}}|u|^{2} \leq \epsilon \int_{-\infty}^{\infty} \tilde{y}^{\prime}\left|u^{\prime}\right|^{2}+B \epsilon^{-1} C^{-2} \omega_{0}^{-2} \int_{-\infty}^{\infty} \tilde{y}^{\prime} \omega_{0}^{2}|u|^{2} .
\end{aligned}
$$

Hence, taking $\epsilon$ sufficiently small and then $C=C\left(\tilde{a}_{0}\right)$ sufficiently large gives us the estimate

$$
b \int_{-\infty}^{\infty}\left(\tilde{y}^{\prime}\left|u^{\prime}\right|^{2}+\tilde{y}^{\prime} \omega_{0}^{2}|u|^{2}\right) \leq\left(\left|u^{\prime}\right|^{2}+\left(\omega-\omega_{+} m\right)^{2}|u|^{2}\right)_{r=r_{+}}-\int_{-\infty}^{\infty} 2 \tilde{y} \operatorname{Re}\left(u^{\prime} \bar{H}\right) .
$$

As in the frequency regime $\mathcal{G} \sharp$, we need to find a large parameter in order to handle the boundary term $\left(\left|u^{\prime}\right|^{2}+\left(\omega-\omega_{+} m\right)^{2}|u|^{2}\right)_{r=r_{+}}$. We employ a $Q^{h}$ current where

$$
\begin{gathered}
h=0 \text { for } r \in\left[r_{+}, R_{1}\right], \quad h^{\prime \prime}=c \tilde{y}^{\prime} \omega_{0}^{2} \text { for } r \in\left[R_{1}, R_{2}\right], \quad h^{\prime \prime}=0 \text { for } r \in\left[R_{2}, R_{3}\right], \\
\left|h^{\prime}\right| \leq \frac{B R_{3} p}{r} \text { for } r \in\left[R_{3}, e^{p^{-1}} R_{3}\right], \quad\left|h^{\prime \prime}\right| \leq \frac{B R_{3} p}{r^{2}} \text { for } r \in\left[R_{3}, e^{p^{-1}} R_{3}\right], \\
h=0 \text { for } r \in\left[e^{p^{-1}} R_{3}, \infty\right) .
\end{gathered}
$$

Note that one may easily construct an $h$ satisfying (137), (138) and (139).

In order to help orient the reader for the estimates below, let us briefly describe the rationale behind the construction of $h$. First of all, the $Q^{h}$ current gives a good estimate when $h$ is positive, $h\left(V-\omega^{2}\right)$ is positive and if the error terms from the $-\frac{1}{2} h^{\prime \prime}$ term can be controlled. Since $V-\omega^{2}$ is only positive for large enough $r$, we set $h$ to be 0 for $r \leq R_{1}$. In order for $h$ to become non-zero, it is necessary for $-\frac{1}{2} h^{\prime \prime}<0$. Thus, the definition of $h$ on $\left[R_{1}, R_{2}\right]$ is motivated by the desire to increase $h$ as fast as possible while still being able to absorb the error term $-\frac{1}{2} h^{\prime \prime}$ with the estimate (136). This successfully produces a positive $h$, but we still need to find a large parameter. In the region $\left[R_{2}, R_{3}\right]$ we achieve this by setting $h^{\prime \prime}=0$, and then letting $h$ grow linearly. Since we have taken $\omega^{2}$ small enough so that $V-\omega^{2}$ is positive on $\left[R_{2}, R_{3}\right]$, by taking $R_{3}$ very large we can arrange for $h$ to be as large as we wish. The crucial estimates for absorbtion of the boundary term $|u(-\infty)|^{2}$ are

$$
\begin{gathered}
r \in\left[R_{2}, R_{3}\right] \Rightarrow b c \omega_{0}^{2} \exp \left(-B C R_{1}\right)\left(r-R_{1}\right) \leq h \leq B R_{3}, \\
r \in\left[R_{2}, R_{3}\right] \Rightarrow h^{-1} \leq B\left(V-\omega^{2}\right) h,
\end{gathered}
$$

see the estimates (150), (151) and (152). Now that we have succeeded in finding a large parameter to absorb the boundary term, we need to take $h$ back down to 0 . Keeping in mind that $p R_{3} \ll 1$, the choice of $h$ on $\left[R_{3}, e^{p^{-1}} R_{3}\right]$ is motivated by the desire to take $h$ down to 0 in a such a way that the error term $-\frac{1}{2} h^{\prime \prime}$ is as small as possible. See estimates (145), (146) and (147) for the details of how these error terms are dealt with. 
We now turn to the specifics. Applying the current $Q^{h}$ gives

$$
\begin{aligned}
& \int_{-\infty}^{\infty}\left(\left(b \tilde{y}^{\prime}+h\right)\left|u^{\prime}\right|^{2}+\left(b \tilde{y}^{\prime} \omega_{0}^{2}+h\left(V-\omega^{2}\right)-\frac{1}{2} h^{\prime \prime}\right)|u|^{2}\right) \\
& \quad \leq\left(\left|u^{\prime}\right|^{2}+\left(\omega-\omega_{+} m\right)^{2}|u|^{2}\right)_{r=r_{+}}-\int_{-\infty}^{\infty}\left(2 \tilde{y} \operatorname{Re}\left(u^{\prime} \bar{H}\right)+h \operatorname{Re}(u \bar{H})\right) .
\end{aligned}
$$

Recall that we explicitly required that $\omega_{\text {low }}$ be sufficiently small so that in particular $V-\omega^{2}$ is positive on $\left[R_{1}, e^{p^{-1}} R_{3}\right]$. Given this, the only negative terms on the left hand side of this estimate come from the $-\frac{1}{2} h^{\prime \prime}$ term on the intervals $\left[R_{1}, R_{2}\right]$ and $\left[R_{3}, e^{p^{-1}} R_{3}\right]$. By construction of $h$ and the assumption that $c \ll 1$, the negative terms on $\left[R_{1}, R_{2}\right]$ can be controlled by the $b y^{\prime} \omega_{0}^{2}|u|^{2}$ term. Therefore, we have

$$
\begin{aligned}
& b \int_{-\infty}^{\infty}\left(\left(\tilde{y}^{\prime}+h\right)\left|u^{\prime}\right|^{2}+\left(\tilde{y}^{\prime} \omega_{0}^{2}+h\left(V-\omega^{2}\right)\right)|u|^{2}\right) \\
& \quad \leq\left(\left|u^{\prime}\right|^{2}+\left(\omega-\omega_{+} m\right)^{2}|u|^{2}\right)_{r=r_{+}}+B p R_{3} \int_{R_{3}}^{e^{p^{-1}} R_{3}}|u|^{2} r^{-2}-\int_{-\infty}^{\infty}\left(2 \tilde{y} \operatorname{Re}\left(u^{\prime} \bar{H}\right)+h \operatorname{Re}(u \bar{H})\right) .
\end{aligned}
$$

The left hand side now is sufficiently strong to absorb the boundary term on the right hand side in a similar fashion as in the $\mathcal{G}^{\sharp}$ regime, i.e. by an application of the current $\chi Q^{K}$ for a suitable cutoff $\chi$. However, we still need to address the term $B p R_{3} \int_{R_{3}}^{e^{p^{-1}} R_{3}}|u|^{2} r^{-2}$. For this we use a $4^{y}$ current with a function $y$ which is determined by

$$
\begin{gathered}
y=0 \text { for } r \in\left[r_{+}, R_{2}\right], \quad y^{\prime}=\frac{h}{2} \text { for } r \in\left[R_{2}, R_{3}\right], \\
y=\frac{r-R_{3}}{R_{3}^{2}}+\frac{1}{2} \int_{R_{2}}^{R_{3}} h \text { for } r \in\left[R_{3}, e^{p^{-1}} R_{3}\right], \\
y=\frac{e^{p^{-1}} R_{3}-R_{3}}{R_{3}^{2}}+\frac{1}{2} \int_{R_{2}}^{R_{3}} h \text { for } r \in\left[e^{p^{-1}} R_{3}, \infty\right) .
\end{gathered}
$$

We obtain

$$
\begin{aligned}
\int_{R_{2}}^{\infty} & \left(y^{\prime}\left|u^{\prime}\right|^{2}+\left(y^{\prime} \omega^{2}-(y V)^{\prime}\right)|u|^{2}\right) \\
& =\left(\frac{e^{p^{-1}} R_{3}-R_{3}}{R_{3}^{2}}+\frac{1}{2} \int_{R_{2}}^{R_{3}} h\right)\left(\left|u^{\prime}\right|^{2}+\omega^{2}|u|^{2}\right)_{r=\infty}-\int_{R_{2}}^{\infty} 2 y \operatorname{Re}\left(u^{\prime} \bar{H}\right) .
\end{aligned}
$$

Observe that $r \in\left[R_{3}, e^{p^{-1}} R_{3}\right]$ implies

$$
\begin{aligned}
-\frac{d}{d r}(y V) & =-\left(R_{3}^{-2}\left(\frac{\Lambda}{r^{2}}+O\left(r^{-3}\right)\right)+\left(\frac{r-R_{3}}{R_{3}^{2}}+\frac{1}{2} \int_{R_{2}}^{R_{3}} h\right)\left(\frac{-2 \Lambda}{r^{3}}+O\left(r^{-4}\right)\right)\right) \\
& \geq R_{3}^{-2} \frac{\Lambda}{r^{2}}+R_{3}^{-1} O\left(r^{-3}\right)+\left(\frac{1}{2} \int_{R_{2}}^{R_{3}} h\right) \frac{2 \Lambda}{r^{3}} \geq b R_{3}^{-2} r^{-2} .
\end{aligned}
$$

We have used that

$$
\int_{R_{2}}^{R_{3}} h \geq b c \omega_{0}^{2} \exp \left(-B C R_{1}\right)\left(R_{3}^{2}-R_{2}^{2}-R_{1}\right),
$$

and that $R_{3}$ has been chosen to dominate $R_{2}+c \omega_{0}^{-2} \exp \left(B C R_{1}\right)$. Of course, $\frac{d}{d r^{*}}(y V)=\left(1+O\left(r^{-1}\right)\right) \frac{d}{d r}(y V)$. We conclude that

$$
-(y V)^{\prime} \geq b R_{3}^{-2} r^{-2}
$$


Next, keeping in mind that $p \ll R_{3}^{-3}$ and that $R_{1}$ sufficiently large implies that $V^{\prime}<0$ for $r \in\left[R_{1}, \infty\right)$, we may add (145) to (141) to obtain

$$
\begin{gathered}
b \int_{-\infty}^{\infty}\left(\left(\tilde{y}^{\prime}+h\right)\left|u^{\prime}\right|^{2}+\left(b \tilde{y}^{\prime} \omega_{0}^{2}+h\left(V-\omega^{2}\right)\right)|u|^{2}\right) \\
\leq\left(\left|u^{\prime}\right|^{2}+\left(\omega-\omega_{+} m\right)^{2}|u|^{2}\right)_{r=r_{+}}+\left(\frac{e^{p^{-1}} R_{3}-R_{3}}{R_{3}^{2}}+\int_{R_{2}}^{R_{3}} h\right)\left(\left|u^{\prime}\right|^{2}+\omega^{2}|u|^{2}\right)_{r=\infty} \\
\quad-\int_{-\infty}^{\infty}\left(2 \tilde{y} \operatorname{Re}\left(u^{\prime} \bar{H}\right)+h \operatorname{Re}(u \bar{H})+2 y \operatorname{Re}\left(u^{\prime} \bar{H}\right)\right) .
\end{gathered}
$$

Lastly, it remains to absorb the boundary terms on the right hand side. We start with the horizon term. Let $\chi_{1}$ be a smooth function such that

$$
\begin{gathered}
\chi_{1}=1 \text { for } r \in\left[r_{+}, R_{2}\right], \quad \chi_{1}=0 \text { for } r \in\left[R_{3}, \infty\right), \\
\left|\chi_{1}^{\prime}\right| \leq B\left(R_{3}-R_{2}\right)^{-1}, \quad\left|\chi_{1}\right| \leq B .
\end{gathered}
$$

We have

$$
\begin{aligned}
\left(\left|u^{\prime}\right|^{2}+\left(\omega-\omega_{+} m\right)^{2}|u|^{2}\right)_{r=r_{+}} & =2 \int_{-\infty}^{\infty}\left(\chi_{1} \mathrm{Q}^{K}\right)^{\prime} \\
& =\left(\omega-\omega_{+} m\right) \int_{R_{2}}^{R_{3}} \chi_{1}^{\prime} \operatorname{Im}\left(u^{\prime} \bar{u}\right)+\left(\omega-\omega_{+} m\right) \int_{-\infty}^{\infty} \chi_{1} \operatorname{Im}(H \bar{u}) .
\end{aligned}
$$

Now, recall that $r \in\left[R_{2}, R_{3}\right]$ implies that $h \geq b c \omega_{0}^{2} \exp \left(-B C R_{1}\right)\left(r-R_{1}\right)$ which in turn implies $h\left(V-\omega^{2}\right) \geq b c \omega_{0}^{2} \exp \left(-B C R_{1}\right) r^{-1}-B R_{1} R_{2}^{-1} r^{-1}-\omega_{\text {low }}^{2} R_{3} \geq b c \omega_{0}^{2} \exp \left(-B C R_{1}\right) r^{-1}$. Thus, keeping in mind that $R_{1} R_{2}^{-1} \ll h\left(V-\omega^{2}\right)$ on $\left[R_{2}, R_{3}\right]$, we conclude that

$$
r \in\left[R_{2}, R_{3}\right] \Rightarrow h^{-1} \leq B h\left(V-\omega^{2}\right) .
$$

Thus,

$$
\begin{aligned}
& \left|\int_{R_{2}}^{R_{3}} \chi_{1}^{\prime} \operatorname{Im}\left(u^{\prime} \bar{u}\right)\right| \leq B\left(R_{3}-R_{2}\right)^{-1} \int_{R_{2}}^{R_{3}}\left|u^{\prime}\right||u| \\
& \quad \leq B\left(R_{3}-R_{2}\right)^{-1} \int_{R_{2}}^{R_{3}}\left(h\left|u^{\prime}\right|^{2}+h^{-1}|u|^{2}\right) \leq B\left(R_{3}-R_{2}\right)^{-1} \int_{R_{2}}^{R_{3}}\left(h\left|u^{\prime}\right|^{2}+h\left(V-\omega^{2}\right)|u|^{2}\right) .
\end{aligned}
$$

Hence, we may combine (150), (147) and (151) to obtain

$$
\begin{aligned}
b \int_{-\infty}^{\infty}( & \left.\left(\tilde{y}^{\prime}+h\right)\left|u^{\prime}\right|^{2}+\left(\tilde{y}^{\prime} \omega_{0}^{2}+h\left(V-\omega^{2}\right)\right)|u|^{2}\right) \\
\leq & \left(\frac{e^{p^{-1}} R_{3}-R_{3}}{R_{3}^{2}}+\int_{R_{2}}^{R_{3}} h\right)\left(\left|u^{\prime}\right|^{2}+\omega^{2}|u|^{2}\right)_{r=\infty} \\
& \quad-\int_{-\infty}^{\infty}\left(2 \tilde{y} \operatorname{Re}\left(u^{\prime} \bar{H}\right)+2 \chi_{1}\left(\omega-\omega_{+} m\right) \operatorname{Im}(H \bar{u})+h \operatorname{Re}(u \bar{H})+2 y \operatorname{Re}\left(u^{\prime} \bar{H}\right)\right) .
\end{aligned}
$$

Now we shall handle the boundary term at $\infty$. Let $\chi_{2}$ be a smooth function such that

$$
\chi_{2}=1 \text { for } r \in\left[R_{2}, \infty\right), \quad \chi_{2}=0 \text { for } r \in\left[r_{+}, R_{1}\right], \quad\left|\chi_{2}\right| \leq B .
$$

Then, we have

$$
\begin{aligned}
& \frac{E}{2}\left(\frac{e^{p^{-1}} R_{3}-R_{3}}{R_{3}^{2}}+\int_{R_{2}}^{R_{3}} h\right)\left[\left|u^{\prime}\right|^{2}+\omega^{2}|u|^{2}\right]_{r=\infty}=E\left(\frac{e^{p^{-1} R_{3}-R_{3}}}{R_{3}^{2}}+\int_{R_{2}}^{R_{3}} h\right) \int_{-\infty}^{\infty}\left(\chi_{2} \mathrm{Q}^{T}\right)^{\prime} \\
& =E\left(\frac{e^{p^{-1}} R_{3}-R_{3}}{R_{3}^{2}}+\int_{R_{2}}^{R_{3}} h\right) \omega \int_{R_{1}}^{R_{2}} \chi_{2}^{\prime} \operatorname{Im}\left(u^{\prime} \bar{u}\right)+E\left(\frac{e^{p^{-1} R_{3}-R_{3}}}{R_{3}^{2}}+\int_{R_{2}}^{R_{3}} h\right) \omega \int_{-\infty}^{\infty} \chi_{2} \operatorname{Im}(H \bar{u}) .
\end{aligned}
$$


We have

$$
\begin{aligned}
& E\left(\frac{e^{p^{-1}} R_{3}-R_{3}}{R_{3}^{2}}+\int_{R_{2}}^{R_{3}} h\right)\left|\omega \int_{R_{1}}^{R_{2}} \chi_{2}^{\prime} \operatorname{Im}\left(u^{\prime} \bar{u}\right)\right| \\
& \quad \leq B E \omega_{0}^{-2} \exp \left(B C R_{2}\right)\left(\frac{e^{p^{-1}} R_{3}-R_{3}}{R_{3}^{2}}+\int_{R_{2}}^{R_{3}} h\right) \omega_{\text {low }} \int_{R_{1}}^{R_{2}}\left(\tilde{y}^{\prime}\left|u^{\prime}\right|^{2}+\omega_{0}^{2} \tilde{y}^{\prime}|u|^{2}\right) .
\end{aligned}
$$

Thus, using that $E \geq 2$, we may combine (154) and (152) and obtain

$$
\begin{aligned}
& b \int_{-\infty}^{\infty}\left(\left(\tilde{y}^{\prime}+h\right)\left|u^{\prime}\right|^{2}+\left(\tilde{y}^{\prime} \omega_{0}^{2}+h\left(V-\omega^{2}\right)\right)|u|^{2}\right) \\
& \leq \int_{-\infty}^{\infty}\left(-2 \tilde{y} \operatorname{Re}\left(u^{\prime} \bar{H}\right)-E y(\infty) \chi_{2} \omega \operatorname{Im}(H \bar{u})-2 \chi_{1}\left(\omega-\omega_{+} m\right) \operatorname{Im}(H \bar{u})-h \operatorname{Re}(u \bar{H})-2 y \operatorname{Re}\left(u^{\prime} \bar{H}\right)\right) .
\end{aligned}
$$

At this point, it is clear that we may rescale the functions $\tilde{y}, h$ and $y$ by an $\tilde{a}_{0}$ dependent constant so that $y$ is identically 1 for $r^{*} \geq R_{\infty}^{*}$.

8.7.4. The subrange $|\omega| \geq \omega_{\text {low }}$ (the non-stationary subcase). We turn finally to our last frequency range. It is only this range which gives rise to the term $1_{\left\{\omega_{\text {low }} \leq|\omega| \leq \omega_{\text {high }}\right\} \cap\left\{\Lambda \leq \epsilon_{\text {width }}^{-1} \omega_{\text {high }}^{2}\right\}}|u(-\infty)|^{2}$ on the right hand side of (69) in the statement of Theorem 8.1

Let $(\omega, m, \Lambda) \in \mathcal{G}_{b}$ where $|\omega| \geq \omega_{\text {low }}$. When the final choices of $\omega_{\text {high }} \epsilon_{\text {width }}, \omega_{\text {low }}$ have been made, we will set the functions $f, h, \tilde{y}, \hat{y}$ and $\chi_{1}$ together with the trapping parameter $r_{\text {trap }}$ to be 0 . The remaining function $y$ and desired coercivity property is given by

Proposition 8.7.4. Let $a_{0}<M$. Then, for all $\omega_{\text {high }}>0, \epsilon_{\text {width }}>0, \omega_{\text {low }}>0, E \geq 2$, for all $R_{\infty}$ sufficiently large depending on $\omega_{\text {high }}, \omega_{\text {low }}, 0 \leq a \leq a_{0}$, and for all $(\omega, m, \Lambda) \in \mathcal{G}_{b}\left(\omega_{\text {high }}, \epsilon_{\text {width }}\right)$ such that $|\omega| \geq \omega_{\text {low }}$, there exists a function $y$ satisfying the uniform bounds

$$
\begin{gathered}
|y| \leq B, \\
y=1 \text { for } r^{*} \geq R_{\infty}^{*},
\end{gathered}
$$

such that, for all smooth solutions $u$ to the radial o.d.e. (44) with right hand side $H$, satisfying moreover the boundary conditions (511) and (52), we have

$$
\begin{aligned}
b\left(\omega_{\text {low }}, \omega_{\text {high }}, \epsilon_{\text {width }}\right) \int_{R_{-}^{*}}^{R_{+}^{*}} & \left(\left|u^{\prime}\right|^{2}+|u|^{2}\right) \\
& \leq B\left(\left|\omega\left(\omega-\omega_{+} m\right)\right||u|^{2}\right)_{r=r_{+}}-\int_{-\infty}^{\infty}\left(2 y \operatorname{Re}\left(u^{\prime} \bar{H}\right)-E \omega \operatorname{Im}(\bar{H} u)\right) .
\end{aligned}
$$

Proof. As in the previous section we will treat the superradiant and non-superradiant frequencies concurrently. However, as previously discussed, it is only for the sake of the superradiant frequencies for which we include the first term on the right hand side of the estimates of the proposition.

Our current will be of the form

for

$$
\mathrm{Q}=\iota^{y}-E \mathrm{Q}^{T}
$$

$$
y\left(r^{*}\right):=\exp \left(-C \int_{r^{*}}^{\infty} \chi_{R_{\infty}^{*}} r^{-2} d r\right),
$$

where $C=C\left(\omega_{\text {low }}, \omega_{\text {high }}, \epsilon_{\text {width }}\right)$ is a sufficiently large constant. The function $\chi_{R_{\infty}^{\star}}$ is a smooth function which is identically 1 on $\left[r_{+}, R_{\infty}-1\right)$ and identically 0 on $\left[R_{\infty}, \infty\right)$. Note that $\left.y\right|_{r^{*} \geq R_{\infty}^{*}}=1$ and $y(-\infty)=0$. Applying the current $\mathrm{Q}$ gives

$$
\begin{aligned}
\int_{-\infty}^{\infty} & \left(y^{\prime}\left|u^{\prime}\right|^{2}+\left(y^{\prime} \omega^{2}-(y V)^{\prime}\right)|u|^{2}\right)-\left(\left|u^{\prime}\right|^{2}+(1-E) \omega^{2}|u|^{2}\right)_{r=\infty} \\
& =\left(\omega\left(\omega-\omega_{+} m\right)|u|^{2}\right)_{r=r_{+}}-\int_{-\infty}^{\infty}\left(2 y \operatorname{Re}\left(u^{\prime} \bar{H}\right)+E \omega \operatorname{Im}(u \bar{H})\right) .
\end{aligned}
$$


Let $R_{\infty}$ be sufficiently large and $R_{6}$ be chosen such that $1 \ll R_{6} \ll R_{\infty}-1$. Then, let $\chi_{2}$ be a smooth function such that

$$
\begin{gathered}
\chi_{2}=1 \text { for } r \in\left[r_{+}, R_{6}\right], \quad \chi_{2}=0 \text { for } R \in\left[R_{\infty}-1, \infty\right), \\
\left|\chi_{2}^{\prime}\right| \leq B\left(R_{\infty}-R_{6}\right)^{-1}, \quad\left|\chi_{2}\right| \leq B .
\end{gathered}
$$

Then set $V_{\leq}:=\chi_{2} V$ and $V_{\geq}=\left(1-\chi_{2}\right) V$. Of course, we have $V=V_{\leq}+V_{\geq}$. For $V_{\leq}$we have

$$
\begin{aligned}
\left.\left|\int_{-\infty}^{\infty}\left(y V_{\leq}\right)^{\prime}\right| u\right|^{2} \mid & =2\left|\int_{-\infty}^{\infty} y V_{\leq} \operatorname{Re}\left(u^{\prime} \bar{u}\right)\right| \\
& \leq \epsilon \int_{-\infty}^{\infty} y^{\prime}\left|u^{\prime}\right|^{2}+B \epsilon^{-1} \int_{-\infty}^{\infty} y^{\prime} \omega^{2} \frac{y^{2} V_{\leq}^{2}}{\left(y^{\prime}\right)^{2} \omega^{2}}|u|^{2} \\
& =\epsilon \int_{-\infty}^{\infty} y^{\prime}\left|u^{\prime}\right|^{2}+B\left(\omega_{\text {high }}, \epsilon_{\text {width }}\right) \epsilon^{-1} \int_{-\infty}^{\infty} y^{\prime} \omega^{2} \frac{V_{\leq}^{2}}{C^{2} r^{-4} \omega^{2}}|u|^{2} \\
& \leq \epsilon \int_{-\infty}^{\infty} y^{\prime}\left|u^{\prime}\right|^{2}+B\left(\omega_{\text {high }}, \epsilon_{\text {width }}\right) \epsilon^{-1} C^{-2} \omega^{-2} \int_{-\infty}^{\infty} y^{\prime} \omega^{2}|u|^{2},
\end{aligned}
$$

while for $V_{\geq}$we have

$$
\begin{aligned}
& -\int_{-\infty}^{\infty}\left(y V_{\geq}\right)^{\prime}|u|^{2}=\int_{-\infty}^{\infty}\left(-y^{\prime} V_{\geq}-y V_{\geq}^{\prime}\right)|u|^{2} \\
& \geq \int_{-\infty}^{\infty}\left(-B\left(\omega_{\text {high }}, \epsilon_{\text {width }}\right) R_{6}^{-2} \omega^{-2}\left(y^{\prime} \omega^{2}\right)\right. \\
& \left.-B\left(\omega_{\text {high }}, \epsilon_{\text {width }}\right)\left(R_{\infty}-R_{6}\right)^{-1} 1_{\operatorname{supp}\left(\chi_{2}^{\prime}\right)} y V+b y\left(1-\chi_{2}\right) r^{-3}\right)|u|^{2} \\
& \geq-B\left(\omega_{\text {high }}, \epsilon_{\text {width }}\right) \max \left(R_{\infty}^{-2} \omega^{-2},\left(R_{\infty}-R_{6}\right)^{-1}\right) \int_{-\infty}^{\infty}\left(y^{\prime} \omega^{2}\right)|u|^{2} .
\end{aligned}
$$

It is now clear that choosing $C, R_{\infty}$ and $R_{\infty}-R_{6}$ sufficiently large depending on $\omega_{\text {low }}$ or $\omega_{\text {low }}$ and $\omega_{\text {high }}$ and $\epsilon_{\text {width }}$ and combining (157), (161) and (162) will imply the proposition.

8.8. Putting everything together. In this section we will combine the propositions of the above sections to prove Theorem 8.1

First of all, keeping Lemma 8.1.1 in mind, we observe that for any choice of $\omega_{\text {high }}$ and $\epsilon_{\text {width }}$, every admissible frequency triple $(\omega, m, \Lambda)$ lies in exactly one of the frequency ranges: $\mathcal{G}_{b}\left(\omega_{\text {high }}, \epsilon_{\text {width }}\right)$, $\mathcal{G}_{\hbar}\left(\omega_{\text {high }}, \epsilon_{\text {width }}\right), \mathcal{G}_{\natural}\left(\omega_{\text {high }}, \epsilon_{\text {width }}\right), \mathcal{G}_{\sharp}\left(\omega_{\text {high }}, \epsilon_{\text {width }}\right), \mathcal{G}^{\sharp}\left(\omega_{\text {high }}, \epsilon_{\text {width }}\right)$. Thus, it only remains to choose the constants $\epsilon_{\text {width }}, E, \omega_{\text {high }}, \omega_{\text {low }}, \tilde{a}_{0}$ and $R_{\infty}$ in the correct order so that it is possible to apply simultaneously all of the above propositions.

The first constant we fix is a sufficiently small $\epsilon_{\text {width }}$, consistent with the requirements of Propositions 8.4.1 and 8.5.1 Then, depending on the choice of $\epsilon_{\text {width }}$, for all large enough $\omega_{\text {high }}, R_{\infty}$ and $E$ we may apply Propositions 8.4.1, 8.5.1 and, in addition, Proposition 8.6.1, corresponding to the frequency regimes $\mathcal{G}_{\sharp}, \mathcal{G}_{\hbar}$ and $\mathcal{G}_{\natural}$. Now we fix the choice of $E$ consistent with the above requirement. Then, depending on this choice of $E$, for all large enough $\omega_{\text {high }}$ and $R_{\infty}$ we may apply, in addition to the above Propositions, also Proposition 8.3.1 corresponding to the frequency regime $\mathcal{G} \sharp$. Finally, we fix the constant $\omega_{\text {high }}$ consistent also with this requirement.

Since $\epsilon_{\text {width }}$ and $\omega_{\text {high }}$ are both fixed, the frequency ranges $\mathcal{G}_{\sharp}, \mathcal{G}_{\hbar}, \mathcal{G}_{\mathfrak{h}}, \mathcal{G}^{\sharp}, \mathcal{G}_{b}$ are now determined.

We still must determine the four subranges of $\mathcal{G}_{b}$ which depend on additional parameters $\omega_{\text {low }}$ and $\tilde{a}_{0}$, and make our final choice of $R_{\infty}$.

We choose first $\tilde{a}_{0}$ and $\omega_{\text {low }}$ sufficiently small so that for $R_{\infty}$ sufficiently large, we can apply Propositions 8.7.1 and 8.7.2 We then fix our choice of $\tilde{a}_{0}$. Then chose sufficiently small $\omega_{\text {low }}$ depending on $\tilde{a}_{0}$, and note that for sufficiently large $R_{\infty}$ depending on $\tilde{a}_{0}$ we may apply (in addition to all previous Propositions) also Proposition 8.7.3. Finally, choose $R_{\infty}$ so that we may apply, in addition to all the previous Propositions, also Proposition 8.7.4. 
With these choices, all frequency ranges are determined so as to indeed simultaneously satisfy the assumptions of Propositions 8.3.1 8.7.4. We now for each frequency range define the functions $f, y, r_{\text {trap }}$, etc., as given in the corresponding Proposition or else set them to 0 (as explained before each statement). The statement of Propositions 8.3.18.7.4 then give Theorem 8.1 for frequencies $(\omega, m, \Lambda)$ in the corresponding range. Since these ranges cover all admissible frequencies, the proof is complete.

8.9. Trapping parameters. We finally define the trapping parameters $s_{ \pm}$which appear in the definition of the degeneration function $\zeta$ (see (13) ) which in turn appears in the statement of Theorem 3.1 .

Definition 8.9.1. Let $0 \leq a_{0}<M$ and let $\omega_{\text {high }}=\omega_{\text {high }}\left(a_{0}, M\right)$ and $\epsilon_{\text {width }}=\epsilon_{\text {width }}\left(a_{0}, M\right)$ be the parameters from Theorem 8.1. We define the trapping parameters $s_{ \pm}$by

$$
\begin{aligned}
& s_{-}\left(a_{0}, M\right) \doteq 3 M-\inf _{0 \leq a \leq a_{0},(\omega, m, \Lambda) \in \mathcal{G}_{\natural}, r_{\text {trap }} \neq 0} r_{\text {trap }}(\omega, m, \Lambda)-\varepsilon\left(a_{0}\right), \\
& s_{+}\left(a_{0}, M\right) \doteq \sup _{0 \leq a \leq a_{0},(\omega, m, \Lambda) \in \mathcal{G}_{\natural} r_{\text {trap }} \neq 0} r_{\text {trap }}(\omega, m, \Lambda)-3 M+\varepsilon\left(a_{0}\right) .
\end{aligned}
$$

where $\varepsilon\left(a_{0}\right)$ is a fixed choice of continuous function such that $\varepsilon(0)=0$ and $\varepsilon\left(a_{0}\right)>0$ for $a_{0}>0$, and such that $s_{ \pm}$satisfy for all $0 \leq a \leq a_{0}$ the relations

$$
r_{+}(a, M)<3 M-s_{-}\left(a_{0}, M\right)<3 M+s_{+}\left(a_{0}, M\right)<\infty .
$$

The proof of Proposition 8.6.1 shows that $\varepsilon\left(a_{0}\right)$ can be chosen ensuring that $s_{ \pm}$, $\varepsilon$ enjoy the properties claimed in the above definition.

Remark 8.9.1. Let us observe that we then necessarily have

$$
\lim _{a_{0} \rightarrow 0} s_{ \pm}\left(a_{0}, M\right)=0, \quad \lim _{a_{0} \rightarrow M} s_{-}\left(a_{0}, M\right)=2 M=3 M-r_{+}(M, M)
$$

From the latter, it follows that we must also have $\lim _{a_{0} \rightarrow M} \varepsilon\left(a_{0}\right)=0$.

Recall the definition of the physical space degeneration function $\zeta$ (see (13)) which in particular required the definition of the points $s_{ \pm}$. It follows from our definition of $r_{\text {trap }}$ in Theorem 8.1 that we now have for all admissible $(\omega, m, \Lambda)$ then $r_{\text {trap }}=0$ or

$$
3 M-s_{-}+\varepsilon\left(a_{0}\right) \leq r_{\text {trap }} \leq 3 M+s_{+}-\varepsilon\left(a_{0}\right)
$$

It follows that for all admissible $(\omega, m, \Lambda)$, we have the uniform bound

$$
b \zeta \leq\left(1-r^{-1} r_{\text {trap }}\right)^{2} .
$$

In particular, the statement of Theorem 8.1 holds with $\left(1-r^{-1} r_{\text {trap }}\right)^{2}$ replaced by $\zeta$. It is this weaker statement that we will in fact apply in the following section.

\section{Summing AND Integrated LOCAL ENERGy DECAY FOR FUtURE-INTEGRABLE SOLUTiOns}

In this section, we will combine the estimates of Sections 4.5. 4.6 and the o.d.e. analysis of Section 8 to prove integrated local energy decay for solutions of the wave equation sufficiently integrable towards the future. We begin by defining this class and stating the main proposition.

9.1. Future-integrable solutions of the wave equation. Let $a_{0}<M,|a| \leq a_{0}$ and let $\psi$ be as in the reduction of Section 4.1. i.e., a solution of the wave equation (1) on $\mathcal{R}_{0}$ arising from smooth compactly supported data at $\Sigma_{0}$. Let $\xi(\tau)$ be smooth function which is 0 in the past of $\Sigma_{0}$ and identically 1 in the future of $\Sigma_{1}$. Then we define

$$
\psi_{\triangleright<} \doteq \xi \psi \text {. }
$$

We have

$$
\square_{g} \psi_{<<}=F \doteq 2 \nabla^{\mu} \xi \nabla_{\mu} \psi+\left(\square_{g} \xi\right) \psi
$$


Definition 9.1.1. Let $|a|<M$ and let $\psi$ be a solution of (11) as in the reduction of Section 4.1. We shall say that $\psi$ is future-integrable if $\psi_{*}$ satisfies Definition [5.1.1.

Note that $\psi_{\times}$by its construction will then automatically satisfy Definitions 5.1 .2

Recall the degeneration function $\zeta$ defined by (13) in Section 2.2.3, and 15, The main result of this section is

Proposition 9.1.1. Let $a_{0}<M,|a| \leq a_{0}$, and let $\psi$ be a future integrable solution of (11). Then, for every $\delta>0$

$$
\begin{aligned}
& \int_{\mathcal{H}_{0}^{+}} \mathbf{J}_{\mu}^{N}[\psi] n_{\mathcal{H}^{+}}^{\mu}+\int_{\mathcal{I}^{+}} \mathbf{J}_{\mu}^{T}[\psi] n_{\mathcal{I}^{+}}^{\mu}+\int_{0}^{\infty} \int_{\Sigma_{\tau}}\left(\left|\tilde{Z}^{*} \psi\right|^{2} r^{-1-\delta}+r^{-3-\delta}|\psi|^{2}+\zeta|T \psi|^{2} r^{-1-\delta}+\zeta|\not \psi \psi|^{2} r^{-1}\right) d \tau \\
& \leq B(\delta) \int_{\Sigma_{0}} \mathbf{J}_{\mu}^{N}[\psi] n_{\Sigma_{0}}^{\mu} .
\end{aligned}
$$

The proof of this proposition will be carried out in Sections 9.29 .7 below. In view of the reduction of Section 4.2 we may assume in this proof that $a \geq 0$, in order to appeal to the the results of Section 8 as stated.

9.2. Finite in time energy estimate. Defining $\psi_{*}$ as above, by Section 5.2 we may apply Carter's separation to the inhomogeneous equation (166) to define the function $u_{m \ell}^{(a \omega)}$. Lemma 5.4.1 implies that for almost every $\omega$, then for all $m, \ell$. the function $H_{m \ell}^{(a \omega)}$ is smooth and $u_{m \ell}^{(a \omega)}$ is a smooth solution to the radial o.d.e. (44) satisfying the boundary conditions (52) and (51). For each such $\omega$ we may apply the estimates of Section 8 to the admissible triples $\left(\omega, m, \Lambda=\Lambda_{m \ell}(a \omega)\right)$ and conclude that Theorem 8.1 holds.

Thus, integrating (69) in $\omega$, summing in $m$ and $\ell$ and applying (165) yields the estimate

$$
\begin{aligned}
& b \int_{-\infty}^{\infty} \sum_{m \ell} \int_{R_{-}^{*}}^{R_{+}^{*}}\left(\left|u^{\prime}\right|^{2}+\left(\zeta\left(\omega^{2}+\Lambda_{m \ell}\right)+1\right)|u|^{2}\right) d r^{*} d \omega \\
& \leq \int_{-\infty}^{\infty} \sum_{m \ell} \int_{-\infty}^{\infty} H \cdot(f, h, y, \chi) \cdot\left(u, u^{\prime}\right) d r^{*} d \omega+\int_{\omega_{\text {low }} \leq|\omega| \leq \omega_{\text {high }}} \sum_{\left\{m \ell: \Lambda \leq \epsilon_{\text {width }}^{-1} \omega_{\text {high }}^{2}\right\}}|u(-\infty)|^{2} d \omega .
\end{aligned}
$$

An application of Plancherel to (167) (see the explicit formulas in Section 5.2.2 and the discussion of the volume form in Section 2.2.6) yields

$$
\begin{aligned}
& b \int_{0}^{\infty} \int_{\Sigma_{\tau} \cap\left[R_{-}, R_{+}\right]}\left(\left|\partial_{r^{*}} \psi_{*}\right|^{2}+\left|\psi_{*}\right|^{2}+\zeta\left|T \psi_{*}\right|^{2}+\left.\zeta|\not| \psi_{*}\right|^{2}\right) d \tau \\
& \leq \int_{-\infty}^{\infty} \sum_{m \ell}\left(\int_{-\infty}^{\infty} H \cdot(f, h, y, \chi) \cdot\left(u, u^{\prime}\right)\right) d \omega+\int_{\omega_{\text {low }} \leq|\omega| \leq \omega_{\text {high }}} \sum_{\left\{m \ell: \Lambda \leq \epsilon_{\text {width }}^{-1} \omega_{\text {high }}^{2}\right\}}|u(-\infty)|^{2} d \omega .
\end{aligned}
$$

Recall that the last term on the right hand side of both of these estimates arises from Section 8.7.4 We further remark that this term would be controlled by the physical space quantity $\int_{\mathcal{H}^{+}(0, \infty)} \mathbf{J}_{\mu}^{N}[\psi] n_{\mathcal{H}^{+}}^{\mu}$, if we had control for the latter-in general, we do not, however! In Section 9.7 we shall exploit the localisation of the integral in $\omega$ to control this using the quantitative mode stability result [59].

The first thing we observe is that $\partial \psi_{\text {se }}$ only differ from $\partial \psi$ when $\tau \in[0,1]$. However, in this region, the energy can simply be controlled by a finite in time energy inequality and a Hardy inequality.

For the $\left|\psi_{<}\right|^{2}$ term, we observe

$$
\int_{0}^{1} \int_{\Sigma_{\tau} \cap\left[R_{-}, R_{+}\right]}|\psi|^{2} \leq B \int_{0}^{1} \int_{\Sigma_{\tau}} \frac{|\psi|^{2}}{r^{2}} \leq B \int_{0}^{1} \int_{\Sigma_{\tau}} \mathbf{J}_{\mu}^{N}[\psi] n_{\Sigma_{\tau}}^{\mu} \leq B \int_{\Sigma_{0}} \mathbf{J}_{\mu}^{N}[\psi] n_{\Sigma_{\tau}}^{\mu},
$$

where we have used a Hardy inequality and a finite in time energy estimate. 
We conclude

$$
\begin{aligned}
& b \int_{0}^{\infty} \int_{\Sigma_{\tau} \cap\left[R_{-}, R_{+}\right]}\left(\left|\partial_{r^{*}} \psi\right|^{2}+|\psi|^{2}+\zeta|T \psi|^{2}+\left.\zeta|\not| \psi\right|^{2}\right) d \tau \\
& \leq \int_{-\infty}^{\infty} \sum_{m \ell}\left(\int_{-\infty}^{\infty} H \cdot(f, h, y, \chi) \cdot\left(u, u^{\prime}\right)\right) d \omega \\
& \quad+\int_{\omega_{\text {low }} \leq|\omega| \leq \omega_{\text {high }}} \sum_{\left\{m \ell: \Lambda \leq \epsilon_{\text {width }}^{-1} \omega_{\text {high }}^{2}\right\}}|u(-\infty)|^{2} d \omega+\int_{\Sigma_{0}} \mathbf{J}_{\mu}^{N}[\psi] n_{\Sigma_{0}}^{\mu} .
\end{aligned}
$$

9.3. Adding in the red-shift. Next, we add a small constant (depending only on $M$ ) times the red-shift estimate of Proposition 4.5.2 to (169). Recalling that $R_{-}=r_{+}+\frac{1}{2}\left(r_{\text {red }}-r_{+}\right)$, we thus obtain

$$
\begin{aligned}
& b \int_{0}^{\infty} \int_{\Sigma_{\tau} \cap\left[r_{+}, R_{+}\right]}\left[\left|\tilde{Z}^{*} \psi_{*}\right|^{2}+\left|\psi_{*}\right|^{2}+\zeta\left|T \psi_{*}\right|^{2}+\zeta\left|\not \psi \psi_{*}\right|^{2}\right] d \tau+b \int_{\mathcal{H}^{+}(0, \infty)} \mathbf{J}_{\mu}^{N}[\psi] n_{\mathcal{H}^{+}}^{\mu} \\
& \leq \int_{-\infty}^{\infty} \sum_{m \ell}\left(\int_{-\infty}^{\infty} H \cdot(f, h, y, \chi) \cdot\left(u, u^{\prime}\right)\right) d \omega \\
& \quad+\int_{\Sigma_{0}} \mathbf{J}_{\mu}^{N}[\psi] n_{\Sigma_{0}}^{\mu}+\int_{\omega_{\text {low }} \leq|\omega| \leq \omega_{\text {high }}} \sum_{\left\{m \ell: \Lambda \leq \epsilon_{\text {widh h }}^{-1} \omega_{\text {high }}^{2}\right\}}|u(-\infty)|^{2} d \omega .
\end{aligned}
$$

9.4. Adding in the large $r$ current. Next, we would like to add in a small constant times the large $r$ estimate of Section 4.6. However, we must be careful because that estimate produces an error proportional to $\int_{\Sigma_{\tau}} \mathbf{J}_{\mu}^{N}[\psi] n_{\Sigma_{\tau}}^{\mu}$, and we do not yet have a uniform energy boundedness statement.

We surmount this difficulty as follows. Since $\psi$ is future-integrable, we know that $\int_{\Sigma_{\tau} \cap\left[r_{+}, R_{+}\right]} \mathbf{J}_{\mu}^{N}[\psi] n_{\Sigma_{\tau}}^{\mu}$, as a function of $\tau$, is in $L_{\tau}^{1}[0, \infty)$. A pigeonhole argument implies that we may find a constant $C(\psi)$ and a dyadic sequence $\left\{\tau_{n}\right\}_{n=1}^{\infty}$ with $\lim _{n \rightarrow \infty} \tau_{n}=\infty$ such that

$$
\int_{\Sigma_{\tau_{n}} \cap\left[r_{+}, R_{+}\right]} \mathbf{J}_{\mu}^{N}[\psi] n_{\Sigma_{\tau_{n}}}^{\mu} \leq \frac{C}{\tau_{n}} .
$$

Note that $R_{+}=2 R_{\text {large }} \geq 4 M$, so that $T$ is timelike in the region $r \geq R_{+}$. Then, a $\mathbf{J}_{\mu}^{T}$ energy estimate implies

$$
\begin{aligned}
\int_{\Sigma_{\tau_{n}}} \mathbf{J}_{\mu}^{N}[\psi] n_{\Sigma}^{\mu} & \leq \int_{\Sigma_{\tau_{n} \cap\left[r_{+}, R_{+}\right]}} \mathbf{J}_{\mu}^{N}[\psi] n_{\Sigma}^{\mu}+B \int_{\Sigma_{\tau_{n}} \cap\left[R_{+}, \infty\right)} \mathbf{J}_{\mu}^{T}[\psi] n_{\Sigma}^{\mu} \\
& \leq B \frac{C}{\tau_{n}}+B \int_{\mathcal{H}^{+}\left(0, \tau_{n}\right)} \mathbf{J}_{\mu}^{N}[\psi] n^{\mu}+B \int_{\Sigma_{0}} \mathbf{J}_{\mu}^{N}[\psi] n^{\mu}
\end{aligned}
$$

Now, combine Proposition 4.6.1 with (172), multiply the result by a sufficiently small constant, and then add the result to (170). In particular, the horizon term on the right hand side of (172) will be multiplied by a small constant, and hence can be absorbed into the left hand side of (170). We obtain

$$
\begin{aligned}
& b \int_{0}^{\tau_{n}} \int_{\Sigma_{\tau}}\left[\left|\tilde{Z}^{*} \psi\right|^{2} r^{-1-\delta}+|\psi|^{2} r^{-3-\delta}+\zeta|T \psi|^{2} r^{-1-\delta}+\zeta|\not \psi|^{2} r^{-1}\right] d \tau \\
& \leq \int_{-\infty}^{\infty} \sum_{m \ell}\left(\int_{-\infty}^{\infty} H \cdot(f, h, y, \chi) \cdot\left(u, u^{\prime}\right)\right) d \omega+B(\delta) \int_{\Sigma_{0}} \mathbf{J}_{\mu}^{N}[\psi] n_{\Sigma_{0}}^{\mu} \\
& +\int_{\omega_{\text {low }} \leq|\omega| \leq \omega_{\text {high }}} \sum_{\left\{m \ell: \Lambda \leq \epsilon_{\text {width }}^{-1} \omega_{\text {high }}^{2}\right\}}|u(-\infty)|^{2} d \omega+\frac{C}{\tau_{n}} .
\end{aligned}
$$


Taking $n \rightarrow \infty$ gives

$$
\begin{aligned}
\int_{0}^{\infty} \int_{\Sigma_{\tau}} & {\left[\left|\tilde{Z}^{*} \psi\right|^{2} r^{-1-\delta}+|\psi|^{2} r^{-3-\delta}+\zeta|T \psi|^{2} r^{-1-\delta}+\zeta|\not \psi|^{2} r^{-1}\right] d \tau } \\
\leq & \int_{-\infty}^{\infty} \sum_{m \ell}\left(\int_{-\infty}^{\infty} H \cdot(f, h, y, \chi) \cdot\left(u, u^{\prime}\right)\right) d \omega \\
& +B(\delta) \int_{\Sigma_{0}} \mathbf{J}_{\mu}^{N}[\psi] n_{\Sigma_{0}}^{\mu}+\int_{\omega_{\text {low }} \leq|\omega| \leq \omega_{\text {high }}} \sum_{\left\{m \ell: \Lambda \leq \epsilon_{\text {width }}^{-1} \omega_{\text {high }}^{2}\right\}}|u(-\infty)|^{2} d \omega .
\end{aligned}
$$

9.5. Boundedness of the energy flux to $\mathcal{I}^{+}$. The estimates of the previous section give in addition an estimate for the energy flux to null infinity

$$
\int_{\mathcal{I}^{+}} \mathbf{J}_{\mu}^{T}[\psi] n_{\mathcal{I}^{+}}^{\mu},
$$

which is well-defined by an easy limiting operation (see [26]) which we omit here.

To bound (175), we only need the easily proven property of its definition, that applying $\mathbf{J}^{T}$ energy estimates outside the ergoregion, (175) can be seen to satisfy

$$
\int_{\mathcal{I}^{+}} \mathbf{J}_{\mu}^{T}[\psi] n_{\mathcal{I}^{+}}^{\mu} \leq \limsup _{n \rightarrow \infty} \int_{\Sigma_{s_{n}}} \mathbf{J}_{\mu}^{N}[\psi] n_{\Sigma_{s_{n}}}^{\mu},
$$

where $\left\{s_{n}\right\}$ is any sequence with $\lim _{n \rightarrow \infty} s_{n}=\infty$.

Now, taking the limit as $n \rightarrow \infty$ in the inequality (172) and then applying (170) gives

$$
\begin{aligned}
\limsup _{n \rightarrow \infty} \int_{\Sigma_{\tau_{n}}} \mathbf{J}_{\mu}^{N}[\psi] n_{\Sigma}^{\mu} & \leq B \int_{\mathcal{H}^{+}(0, \infty)} \mathbf{J}_{\mu}^{N}[\psi] n^{\mu}+B \int_{\Sigma_{0}} \mathbf{J}_{\mu}^{N}[\psi] n^{\mu} \\
& \leq B \int_{\Sigma_{0}} \mathbf{J}_{\mu}^{N}[\psi] n_{\Sigma_{0}}^{\mu} .
\end{aligned}
$$

Then (176) implies

$$
\int_{\mathcal{I}^{+}} \mathbf{J}_{\mu}^{T}[\psi] n_{\mathcal{I}^{+}}^{\mu} \leq \limsup _{n \rightarrow \infty} \int_{\Sigma_{\tau_{n}}} \mathbf{J}_{\mu}^{N}[\psi] n_{\Sigma}^{\mu} \leq B \int_{\Sigma_{0}} \mathbf{J}_{\mu}^{N}[\psi] n_{\Sigma_{0}}^{\mu}
$$

An alternative approach for controlling $\int_{\mathcal{I}^{+}} \mathbf{J}_{\mu}^{N}[\psi] n_{\mathcal{I}^{+}}^{\mu}$ can be found in the proof of the inequality (239) where a cut-off $\mathbf{J}^{T}$ energy estimate is applied and the errors are absorbed with the integrated energy decay statement. Lastly, we note that yet another approach would be to first show that (up to a normalisation constant)

$$
\int_{\mathcal{I}^{+}} \mathbf{J}_{\mu}^{T}[\psi] n_{\mathcal{I}^{+}}^{\mu}=\int_{-\infty}^{\infty} \sum_{m \ell} \omega^{2}|u(\infty)|^{2}
$$

and then use the fact that the estimates of Section 8 give a bound for the right hand side of this equality.

9.6. Error terms associated to the cutoff. In this subsection we will study closely the terms $\int_{-\infty}^{\infty} \sum_{m \ell}\left(\int_{-\infty}^{\infty} H \cdot(f, h, y, \chi) \cdot\left(u, u^{\prime}\right)\right) d \omega$ on the right hand side of (174). Recall that when $r \geq$ $R_{\infty}$, we have arranged for our multipliers to be independent of the frequency parameters or decay exponentially in $r$ (see Remark 8.2). In particular, we may split the error terms associated to the 
cutoff into:

(179)

$$
\begin{aligned}
\int_{-\infty}^{\infty} \sum_{m \ell} & \left(\int_{-\infty}^{\infty} H \cdot(f, h, y, \chi) \cdot\left(u, u^{\prime}\right)\right) d \omega \\
= & \int_{-\infty}^{\infty} \sum_{m \ell}\left(\int_{-\infty}^{\infty} \chi_{R_{\infty}} H \cdot(f, h, y, \chi) \cdot\left(u, u^{\prime}\right)\right) d \omega-\int_{-\infty}^{\infty} \sum_{m \ell}\left(\int_{-\infty}^{\infty} 2\left(1-\chi_{R_{\infty}}\right) \operatorname{Re}\left(u^{\prime} \bar{H}\right)\right) d \omega \\
& +\int_{-\infty}^{\infty} \sum_{m \ell}\left(\int_{-\infty}^{\infty} E \omega\left(1-\chi_{R_{\infty}}\right) \operatorname{Im}(H \bar{u})\right) d \omega \\
& +2 \int_{\omega_{\text {low }} \leq|\omega| \leq \omega_{\text {high }}\left\{m \ell: \Lambda \leq \epsilon_{\text {width }}^{-1} \omega_{\text {high }}^{2}\right\}}\left(\int_{-\infty}^{\infty}\left(1-\chi_{R_{\infty}}\right) \tilde{y} \operatorname{Re}\left(u^{\prime} \bar{H}\right)\right) d \omega
\end{aligned}
$$

Here $\chi_{R_{\infty}}$ is a cutoff which is identically 1 on $\left[r_{+}, R_{\infty}\right]$ and 0 on $\left[R_{\infty}+1, \infty\right)$.

9.6.1. The bounded $r$ error terms. The error terms in the region $\left[r_{+}, R_{\infty}\right]$ are the easiest to deal with. We simply observe that an application of Plancherel (see Sections 5.2 .2 and 2.2.6) , finite in time energy estimates and Hardy inequalities

$$
\begin{aligned}
\mid \int_{-\infty}^{\infty} & \sum_{m \ell}\left(\int_{-\infty}^{\infty} \chi_{R_{\infty}} H \cdot(f, h, y, \chi) \cdot\left(u, u^{\prime}\right)\right) d \omega \mid \\
& \leq B \int_{-\infty}^{\infty} \sum_{m \ell} \int_{-\infty}^{R_{\infty}+1}|H|\left(\left|u^{\prime}\right|+|u|\right) \\
& \leq B \epsilon^{-1} \int_{0}^{\infty} \int_{\Sigma_{\tau} \cap\left[r_{+}, R_{\infty}+1\right)}|F|^{2}+\epsilon \int_{0}^{\infty} \int_{\Sigma_{\tau} \cap\left[r_{+}, R_{\infty}+1\right)}\left(\left|\partial_{r^{*}} \psi\right|^{2}+|\psi|^{2}\right) \\
& \leq B \epsilon^{-1} \int_{0}^{2} \int_{\Sigma_{\tau} \cap\left[r_{+}, \infty\right)} \mathbf{J}_{\mu}^{N}[\psi] n_{\Sigma_{\tau}}^{\mu}+\epsilon \int_{0}^{\infty} \int_{\Sigma_{\tau} \cap\left[r_{+}, R_{\infty}+1\right)}\left(\left|\partial_{r^{*}} \psi\right|^{2}+|\psi|^{2}\right) \\
& \leq B \epsilon^{-1} \int_{\Sigma_{0}} \mathbf{J}_{\mu}^{N}[\psi] n_{\Sigma_{0}}^{\mu}+\epsilon \int_{0}^{\infty} \int_{\Sigma_{\tau} \cap\left[r_{+}, R_{\infty}+1\right)}\left(\left|\partial_{r^{*}} \psi\right|^{2}+|\psi|^{2}\right) .
\end{aligned}
$$

9.6.2. Large $r$ : $\iota^{y}$ error terms. For error terms supported in the $r \in\left[R_{\infty}, \infty\right)$ region we must be careful that lower order terms come with appropriate $r$-weights so that either a Hardy inequality can be applied or they can be absorbed into the left hand side of (174). First of all, an application of Plancherel (see Section 5.2.2) gives

$$
\begin{aligned}
& \int_{-\infty}^{\infty} \sum_{m \ell}\left(\int_{-\infty}^{\infty}\left(1-\chi_{R_{\infty}}\right) \operatorname{Re}\left(u^{\prime} \bar{H}\right)\right) d \omega \\
= & \int_{0}^{\infty} \int_{-\infty}^{\infty} \int_{\mathbb{S}^{2}}\left(1-\chi_{R_{\infty}}\right) \operatorname{Re}\left(\partial_{r^{*}}\left(\left(r^{2}+a^{2}\right)^{1 / 2} \psi_{\odot}\right) \overline{\Delta\left(r^{2}+a^{2}\right)^{-1 / 2} F}\right) \sin \theta d t d r^{*} d \theta d \phi .
\end{aligned}
$$

Recall that

$$
F=\left(r^{2}+a^{2}\right)^{-1} \rho^{2}\left(2 \nabla^{\mu} \xi \nabla_{\mu} \psi+\left(\square_{g} \xi\right) \psi\right) .
$$

For sufficiently large $r, \xi$ is just a function of $t$. Hence,

$$
F=\left(r^{2}+a^{2}\right)^{-1} \rho^{2}\left(2 g^{t t} \dot{\xi} \partial_{t} \psi+2 g^{t \phi} \dot{\xi} \partial_{\phi} \psi+g^{t t} \ddot{\xi} \psi\right) .
$$

Thus, (suppressing the $\sin \theta d t d r^{*} d \theta d \phi$ )

$$
\begin{aligned}
& \int_{0}^{\infty} \int_{-\infty}^{\infty} \int_{\mathbb{S}^{2}}\left(1-\chi_{R_{\infty}}\right) \operatorname{Re}\left(\partial_{r^{*}}\left(\left(r^{2}+a^{2}\right)^{1 / 2} \psi_{<}\right) \overline{\Delta\left(r^{2}+a^{2}\right)^{-1 / 2} F}\right) \\
& =\int_{0}^{\infty} \int_{-\infty}^{\infty} \int_{\mathbb{S}^{2}}\left(1-\chi_{R_{\infty}}\right) \operatorname{Re}\left(\partial_{r^{*}}\left(\left(r^{2}+a^{2}\right)^{1 / 2} \psi_{x}\right) \overline{\Delta\left(r^{2}+a^{2}\right)^{-3 / 2} \rho^{2}\left(2 g^{t t} \dot{\xi} \partial_{t} \psi+2 g^{t \phi} \dot{\xi} \partial_{\phi} \psi\right)}\right) \\
& \quad+\int_{0}^{\infty} \int_{-\infty}^{\infty} \int_{\mathbb{S}^{2}}\left(1-\chi_{R_{\infty}}\right) \operatorname{Re}\left(\partial_{r^{*}}\left(\left(r^{2}+a^{2}\right)^{1 / 2} \psi_{x}\right) \overline{\Delta\left(r^{2}+a^{2}\right)^{-3 / 2} \rho^{2} g^{t t} \ddot{\xi} \psi}\right) .
\end{aligned}
$$


First we consider the term with $g^{t t} \dot{\xi} \partial_{t} \psi$ :

$$
\begin{aligned}
& \left|\int_{0}^{\infty} \int_{-\infty}^{\infty} \int_{\mathbb{S}^{2}}\left(1-\chi_{R_{\infty}}\right) \operatorname{Re}\left(\partial_{r^{*}}\left(\left(r^{2}+a^{2}\right)^{1 / 2} \psi_{\star}\right) \overline{\Delta\left(r^{2}+a^{2}\right)^{-3 / 2} \rho^{2}\left(2 g^{t t} \dot{\xi} \partial_{t} \psi\right)}\right)\right| \\
\leq & B\left|\int_{0}^{\infty} \int_{-\infty}^{\infty} \int_{\mathbb{S}^{2}}\left(1-\chi_{R_{\infty}}\right) \operatorname{Re}\left(\left(\partial_{r^{*}} \psi_{*}\right) \overline{\Delta\left(r^{2}+a^{2}\right)^{-1} \rho^{2}\left(2 g^{t t} \dot{\xi} \partial_{t} \psi\right)}\right)\right| \\
& +B\left|\int_{0}^{\infty} \int_{-\infty}^{\infty} \int_{\mathbb{S}^{2}}\left(1-\chi_{R_{\infty}}\right) \frac{r}{\left(r^{2}+a^{2}\right)^{1 / 2}} \operatorname{Re}\left(\left(\psi_{\odot}\right) \overline{\Delta\left(r^{2}+a^{2}\right)^{-3 / 2} \rho^{2}\left(2 g^{t t} \dot{\xi} \partial_{t} \psi\right)}\right)\right| \\
\leq & B \int_{0}^{1} \int_{\Sigma_{\tau} \cap\left[R_{\infty}, \infty\right)}\left(\mathbf{J}_{\mu}^{N}[\psi] n_{\Sigma_{\tau}}^{\mu}+\frac{|\psi|^{2}}{r^{2}}\right) \leq B \int_{\Sigma_{0}} \mathbf{J}_{\mu}^{N}[\psi] n_{\Sigma_{\tau}}^{\mu} .
\end{aligned}
$$

Keeping in mind that $g^{t \phi}=O\left(r^{-3}\right)$, the term with $g^{t \phi} \dot{\xi} \partial_{\phi} \psi$ can be treated like (183). Finally, recalling that $\partial_{r^{*}} \xi=0$ for $r \geq R_{\infty}$, we have

$$
\begin{aligned}
& \left|\int_{0}^{\infty} \int_{-\infty}^{\infty} \int_{\mathbb{S}^{2}}\left(1-\chi_{R_{\infty}}\right) \operatorname{Re}\left(\partial_{r^{*}}\left(\left(r^{2}+a^{2}\right)^{1 / 2} \psi_{<}\right) \overline{\Delta\left(r^{2}+a^{2}\right)^{-3 / 2} \rho^{2} g^{t t} \ddot{\xi} \psi}\right)\right| \\
& =\left|\int_{0}^{\infty} \int_{-\infty}^{\infty} \int_{\mathbb{S}^{2}}\left(1-\chi_{R_{\infty}}\right) \Delta\left(r^{2}+a^{2}\right)^{-2} \rho^{2} g^{t t} \ddot{\xi} \xi \operatorname{Re}\left(\partial_{r^{*}}\left(\left(r^{2}+a^{2}\right)^{1 / 2} \psi\right) \overline{\left(r^{2}+a^{2}\right)^{1 / 2} \psi}\right)\right| \\
& =\left.\frac{1}{2}\left|\int_{0}^{\infty} \int_{-\infty}^{\infty} \int_{\mathbb{S}^{2}} \partial_{r^{*}}\left(\left(1-\chi_{R_{\infty}}\right) \Delta\left(r^{2}+a^{2}\right)^{-2} \rho^{2} g^{t t} \ddot{\xi} \xi\right)\left(r^{2}+a^{2}\right)\right| \psi\right|^{2} \mid \\
& \leq B \int_{0}^{1} \int_{\Sigma_{\tau} \cap\left[R_{\infty}, \infty\right)} \frac{|\psi|^{2}}{r^{2}} \leq B \int_{\Sigma_{0}} \mathbf{J}_{\mu}^{N}[\psi] n_{\Sigma_{0}}^{\mu} .
\end{aligned}
$$

Combining everything implies

$$
\left|\int_{-\infty}^{\infty} \sum_{m \ell}\left(\int_{-\infty}^{\infty} 2\left(1-\chi_{R_{\infty}}\right) \operatorname{Re}\left(u^{\prime} \bar{H}\right)\right) d \omega\right| \leq B \int_{\Sigma_{0}} \mathbf{J}_{\mu}^{N}[\psi] n_{\Sigma_{0}}^{\mu} .
$$

9.6.3. Large r: $Q^{T}$ error terms. An application of Plancherel (see Section 5.2.2) gives

$$
\begin{aligned}
& \left|\int_{-\infty}^{\infty} \sum_{m \ell}\left(\int_{-\infty}^{\infty} \omega\left(1-\chi_{R_{\infty}}\right) \operatorname{Im}(H \bar{u})\right) d \omega\right| \\
& =\left|\int_{0}^{\infty} \int_{-\infty}^{\infty} \int_{\mathbb{S}^{2}} \Delta\left(1-\chi_{R_{\infty}}\right) \operatorname{Re}\left(\partial_{t} \psi_{<} \bar{F}\right) \sin \theta d t d r^{*} d \theta d \phi\right| .
\end{aligned}
$$

We have

$$
F=\left(r^{2}+a^{2}\right)^{-1} \rho^{2}\left(2 g^{t t} \dot{\xi} \partial_{t} \psi+2 g^{t \phi} \dot{\xi} \partial_{\phi} \psi+g^{t t} \ddot{\xi} \psi\right), \quad \partial_{t} \psi_{<}=\dot{\xi} \psi+\xi \partial_{t} \psi
$$

To ease the notation, let us introduce

$$
W(r, \theta):=\Delta\left(r^{2}+a^{2}\right)^{-1}\left(1-\chi_{R_{\infty}}\right) \rho^{2} .
$$

Then, suppressing the $\sin \theta d t d r^{*} d \theta d \phi$, we have

$$
\begin{aligned}
\mid \int_{0}^{\infty} & \int_{-\infty}^{\infty} \int_{\mathbb{S}^{2}} \Delta\left(1-\chi_{R_{\infty}}\right) \operatorname{Re}\left(\partial_{t} \psi_{<} \bar{F}\right) \mid \\
\leq 2 & \left|\int_{0}^{\infty} \int_{-\infty}^{\infty} \int_{\mathbb{S}^{2}} W(r, \theta) g^{t \phi} \dot{\xi} \operatorname{Re}\left(\left(\dot{\xi} \psi+\xi \partial_{t} \psi\right) \overline{\partial_{\phi} \psi}\right)\right| \\
& +\left|\int_{0}^{\infty} \int_{-\infty}^{\infty} \int_{\mathbb{S}^{2}} W(r, \theta) \operatorname{Re}\left(\left(\dot{\xi} \psi+\xi \partial_{t} \psi\right) \overline{\left(2 g^{t t} \dot{\xi} \partial_{t} \psi+g^{t t} \ddot{\xi} \psi\right)}\right)\right| .
\end{aligned}
$$

Several of the above terms are easy to deal with:

$$
\begin{gathered}
\left|\int_{0}^{\infty} \int_{-\infty}^{\infty} \int_{\mathbb{S}^{2}} W(r, \theta) g^{t \phi} \dot{\xi} \operatorname{Re}\left((\dot{\xi} \psi) \overline{\partial_{\phi} \psi}\right)\right|=\left.\left|\int_{0}^{\infty} \int_{-\infty}^{\infty} \int_{\mathbb{S}^{2}} W(r, \theta) g^{t \phi}(\dot{\xi})^{2} \partial_{\phi}\right| \psi\right|^{2} \mid=0 . \\
\left|\int_{0}^{\infty} \int_{-\infty}^{\infty} \int_{\mathbb{S}^{2}} W(r, \theta) g^{t \phi} \dot{\xi} \xi \operatorname{Re}\left(\left(\partial_{t} \psi\right) \overline{\partial_{\phi} \psi}\right)\right| \leq \int_{0}^{1} \int_{\Sigma_{\tau}} \mathbf{J}_{\mu}^{N}[\psi] n_{\Sigma_{\tau}}^{\mu} \leq \int_{\Sigma_{0}} \mathbf{J}_{\mu}^{N}[\psi] n_{\Sigma_{0}}^{\mu} .
\end{gathered}
$$




$$
\left.2\left|\int_{0}^{\infty} \int_{-\infty}^{\infty} \int_{\mathbb{S}^{2}} W(r, \theta) g^{t t} \xi \dot{\xi}\right| \partial_{t} \psi\right|^{2} \mid \leq \int_{0}^{1} \int_{\Sigma_{\tau}} \mathbf{J}_{\mu}^{N}[\psi] n_{\Sigma_{\tau}}^{\mu} \leq \int_{\Sigma_{0}} \mathbf{J}_{\mu}^{N}[\psi] n_{\Sigma_{0}}^{\mu} .
$$

Combining everything yields

$$
\begin{aligned}
& \left|\int_{-\infty}^{\infty} \sum_{m \ell}\left(\int_{-\infty}^{\infty} \omega\left(1-\chi_{R_{\infty}}\right) \operatorname{Im}(H \bar{u})\right) d \omega\right| \\
& \leq\left|\int_{0}^{\infty} \int_{-\infty}^{\infty} \int_{\mathbb{S}^{2}} W(r, \theta)\left(\operatorname{Re}\left(\dot{\xi} \psi \overline{\left(2 g^{t t} \dot{\xi} \partial_{t} \psi+g^{t t} \ddot{\xi} \psi\right)}\right)+\operatorname{Re}\left(\xi \partial_{t} \psi \overline{g^{t t} \ddot{\xi} \psi}\right)\right)\right|+\int_{\Sigma_{0}} \mathbf{J}_{\mu}^{N}[\psi] n_{\Sigma_{0}}^{\mu} .
\end{aligned}
$$

We now focus on the first term on the right hand side:

$$
\begin{aligned}
& \left|\int_{0}^{\infty} \int_{-\infty}^{\infty} \int_{\mathbb{S}^{2}} g^{t t} W(r, \theta)\left(2(\dot{\xi})^{2} \operatorname{Re}\left(\psi \overline{\partial_{t} \psi}\right)+\dot{\xi} \ddot{\xi}|\psi|^{2}+\xi \ddot{\xi} \operatorname{Re}\left(\partial_{t} \psi \bar{\psi}\right)\right)\right| \\
& =\left|\int_{0}^{\infty} \int_{-\infty}^{\infty} \int_{\mathbb{S}^{2}} g^{t t} W(r, \theta)\left(2(\dot{\xi})^{2} \operatorname{Re}\left(\psi \overline{\partial_{t} \psi}\right)-(\dot{\xi})^{2} \operatorname{Re}\left(\psi \overline{\partial_{t} \psi}\right)+\xi \ddot{\xi} \operatorname{Re}\left(\partial_{t} \psi \bar{\psi}\right)\right)\right| \\
& =\left|\int_{0}^{\infty} \int_{-\infty}^{\infty} \int_{\mathbb{S}^{2}} g^{t t} W(r, \theta)\left((\dot{\xi})^{2} \operatorname{Re}\left(\psi \overline{\partial_{t} \psi}\right)+\xi \ddot{\xi} \operatorname{Re}\left(\partial_{t} \psi \bar{\psi}\right)\right)\right| \\
& =\left|\int_{0}^{\infty} \int_{-\infty}^{\infty} \int_{\mathbb{S}^{2}} g^{t t} W(r, \theta)\left((\dot{\xi})^{2} \operatorname{Re}\left(\psi \overline{\partial_{t} \psi}\right)-(\dot{\xi})^{2} \operatorname{Re}\left(\partial_{t} \psi \bar{\psi}\right)-\xi \dot{\xi} \operatorname{Re}\left(\partial_{t}^{2} \psi \bar{\psi}\right)-\xi \dot{\xi}\left|\partial_{t} \psi\right|^{2}\right)\right| \\
& =\left|\int_{0}^{\infty} \int_{-\infty}^{\infty} \int_{\mathbb{S}^{2}} g^{t t} W(r, \theta)\left(\xi \dot{\xi} \operatorname{Re}\left(\partial_{t}^{2} \psi \bar{\psi}\right)+\xi \dot{\xi}\left|\partial_{t} \psi\right|^{2}\right)\right| \\
& \leq \int_{\Sigma_{0}} \mathbf{J}_{\mu}^{N}[\psi] n_{\Sigma_{0}}^{\mu}+\left|\int_{0}^{\infty} \int_{-\infty}^{\infty} \int_{\mathbb{S}^{2}} g^{t t} W(r, \theta)\left(\xi \dot{\xi} \operatorname{Re}\left(\partial_{t}^{2} \psi \bar{\psi}\right)\right)\right| .
\end{aligned}
$$

Instead of additional integration by parts on this last term, we use that $\psi$ solves the wave equation, which we write out here for reference:

$$
g^{t t} \partial_{t}^{2} \psi=\frac{4 M a r}{\rho^{2} \Delta} \partial_{t, \phi}^{2} \psi-\frac{\Delta-a^{2} \sin ^{2} \theta}{\Delta \rho^{2} \sin ^{2} \theta} \partial_{\phi}^{2} \psi-\frac{r^{2}+a^{2}}{\Delta \rho^{2}} \partial_{r^{*}}\left(\left(r^{2}+a^{2}\right) \partial_{r^{*}} \psi\right)-\frac{1}{\rho^{2} \sin \theta} \partial_{\theta}\left(\sin \theta \partial_{\theta} \psi\right) .
$$

Substituting the right hand side of (189) for $g^{t t} \partial_{t}^{2} \psi$, carrying out a straightforward integration by parts, and applying a finite in time energy inequality shows

$$
\left|\int_{0}^{\infty} \int_{-\infty}^{\infty} \int_{\mathbb{S}^{2}} g^{t t} W(r, \theta)\left(\xi \dot{\xi} \operatorname{Re}\left(\partial_{t}^{2} \psi \bar{\psi}\right)\right)\right| \leq B \int_{\Sigma_{0}} \mathbf{J}_{\mu}^{N}[\psi] n_{\Sigma_{0}}^{\mu} .
$$

Thus, we have shown

$$
\left|\int_{-\infty}^{\infty} \sum_{m \ell}\left(\int_{-\infty}^{\infty} \omega\left(1-\chi_{R_{\infty}}\right) \operatorname{Im}(H \bar{u})\right) d \omega\right| \leq B \int_{\Sigma_{0}} \mathbf{J}_{\mu}^{N}[\psi] n_{\Sigma_{0}}^{\mu}
$$

9.6.4. Large $r$ : $\iota^{\tilde{y}}$ error terms. Since $|\tilde{y}| \leq \exp \left(-b r^{*}\right)$ as $r^{*} \rightarrow \infty$, we may estimate the term $\int_{\omega_{\text {low }} \leq|\omega| \leq \omega_{\text {high }}} \sum_{\left\{m \ell: \Lambda \leq \epsilon_{\text {width }}^{-1} \omega_{\text {high }}^{2}\right\}}\left(\int_{-\infty}^{\infty}\left(1-\chi_{R_{\infty}}\right) \tilde{y} \operatorname{Re}\left(u^{\prime} \bar{H}\right)\right) d \omega$ exactly as in Section 9.6.1. We obtain

$$
\begin{aligned}
& \left|\int_{\omega_{\text {low }} \leq|\omega| \leq \omega_{\text {high }}} \sum_{\left\{m \ell: \Lambda \leq \epsilon_{\text {width }}^{-1} \omega_{\text {high }}^{2}\right\}}\left(\int_{-\infty}^{\infty}\left(1-\chi_{R_{\infty}}\right) \tilde{y} \operatorname{Re}\left(u^{\prime} \bar{H}\right)\right) d \omega\right| \\
& \leq B \epsilon^{-1} \int_{\Sigma_{0}} \mathbf{J}_{\mu}^{N}[\psi] n_{\Sigma_{0}}^{\mu}+\epsilon \int_{0}^{\infty} \int_{\Sigma_{\tau} \cap\left[R_{\infty}, \infty\right)} e^{-b r^{*}}\left(\left|\partial_{r^{*}} \psi\right|^{2}+|\psi|^{2}\right) .
\end{aligned}
$$

9.6.5. Absorbing the error terms. Combining the results of Sections 9.6.1 9.6.2, 9.6.3 and 9.6.4 gives

$$
\begin{aligned}
& \int_{-\infty}^{\infty} \sum_{m \ell}\left(\int_{-\infty}^{\infty} H \cdot(f, h, y, \chi) \cdot\left(u, u^{\prime}\right)\right) d \omega \\
& \quad \leq B \int_{\Sigma_{0}} \mathbf{J}_{\mu}^{N}[\psi] n_{\Sigma_{0}}^{\mu}+\epsilon \int_{0}^{\infty} \int_{\Sigma_{\tau}} r^{-1-\delta}\left(\left|\partial_{r^{*}} \psi\right|^{2}+r^{-2}|\psi|^{2}+\zeta \mathbf{J}_{\mu}^{N}[\psi] n_{\Sigma_{\tau}}^{\mu}\right) .
\end{aligned}
$$


Taking $\epsilon$ sufficiently small and combining (190) with (174), (170) and (178) implies

$$
\begin{aligned}
& b \int_{\mathcal{H}^{+}(0, \infty)} \mathbf{J}_{\mu}^{N}[\psi] n_{\mathcal{H}^{+}}^{\mu}+b \int_{\mathcal{I}_{0}^{+}} \mathbf{J}_{\mu}^{N}[\psi] n_{\mathcal{I}^{+}}^{\mu} \\
& \quad+b \int_{0}^{\infty} \int_{\Sigma_{\tau}}\left(\left|\partial_{r^{*}} \psi\right|^{2} r^{-1-\delta}+|\psi|^{2} r^{-3-\delta}+\zeta|T \psi|^{2} r^{-1-\delta}+\zeta|\not \psi \psi|^{2} r^{-1}\right) d \tau \\
& \leq B(\delta) \int_{\Sigma_{0}} \mathbf{J}_{\mu}^{N}[\psi] n_{\Sigma_{0}}^{\mu}+\int_{\omega_{\text {low }} \leq|\omega| \leq \omega_{\text {high }}} \sum_{\left\{m \ell: \Lambda \leq \epsilon_{\text {width }}^{-1} \omega_{\text {high }}^{2}\right\}}|u(-\infty)|^{2} d \omega .
\end{aligned}
$$

9.7. The non-stationary bounded frequency horizon term. Finally, we come to the term $\int_{\omega_{\text {low }} \leq|\omega| \leq \omega_{\text {high }}} \sum_{\left\{m \ell: \Lambda \leq \epsilon_{\text {width }}^{-1} \omega_{\text {high }}^{2}\right\}}|u(-\infty)|^{2} d \omega$. Since we do not have a small parameter, we cannot hope to absorb this error term into the left hand side of (191); however, this term has already been dealt with in the context of the quantitative mode stability work [59:

Proposition 9.7.1. Let $\psi$ be a future-integrable solution to (1). Define $u$ by (42) with $\Psi=\psi_{\times}$. Then

$$
\int_{\omega_{\text {low }} \leq|\omega| \leq \omega_{\text {high }}} \sum_{\left\{m \ell: \Lambda \leq \epsilon_{\text {width }}^{-1} \omega_{\text {high }}^{2}\right\}}|u(-\infty)|^{2} d \omega \leq B \int_{\Sigma_{0}} \mathbf{J}_{\mu}^{N}[\psi] n_{\Sigma_{0}}^{\mu} .
$$

Proof. This follows immediately from Theorem 1.9 of [59] if we replace $\Sigma_{0}$ with a hyperboloidal hypersurface $\tilde{\Sigma}_{0}$. For any $1 \ll R$ one can easily find a hyperboloidal hypersurface $\tilde{\Sigma}_{0}$ which agrees with $\Sigma_{0}$ on $\{r \leq R\}$ and which lies to the future of $\Sigma_{0}$. If we choose $R$ large enough so that $T$ is timelike in the region $\{r \geq R\}$, then a $\mathbf{J}_{\mu}^{T}$ energy estimate will immediately imply

$$
\int_{\omega_{\text {low }} \leq|\omega| \leq \omega_{\text {high }}} \sum_{\left\{m \ell: \Lambda \leq \epsilon_{\text {width }}^{-1} \omega_{\text {high }}^{2}\right\}}|u(-\infty)|^{2} d \omega \leq B \int_{\tilde{\Sigma}_{0}} \mathbf{J}_{\mu}^{N}[\psi] n_{\tilde{\Sigma}_{0}}^{\mu} \leq B \int_{\Sigma_{0}} \mathbf{J}_{\mu}^{N}[\psi] n_{\Sigma_{0}}^{\mu} .
$$

Remark 9.7.1. We observe that the appeal to [59] is not necessary in the case of a $M$ or if $\psi$ is only supported on sufficiently high azimuthal frequencies.

Combining (191) with Proposition 9.7.1 finishes the proof of Proposition 9.1.1.

9.8. An inhomogeneous estimate. In Sections 10 and 11 we will need to consider future-integrable solutions $\Psi$ to the inhomogeneous wave equation $\square_{g_{a, M}} \Psi=F$.

Let us first generalise the definition of future-integrability to apply to general smooth $\Psi$.

Definition 9.8.1. With cutoff $\xi(\tau)$ as in Section 9.1, we say that $\Psi: \mathcal{R}_{0} \rightarrow \mathbb{R}$ is future-integrable if $\xi \Psi$ satisfies Definitions 5.1.1 and 5.1.2.

Proposition 9.8.1. Let $\Psi$ be a future integrable solution of the inhomogeneous wave equation $\square_{g_{a, M}} \Psi=F$. Then, for every $\delta>0$ and $\epsilon>0$,

$$
\begin{aligned}
& \int_{\mathcal{H}^{+}(0, \infty)} \mathbf{J}_{\mu}^{N}[\Psi] n_{\mathcal{H}^{+}}^{\mu}+\int_{\mathcal{I}^{+}} \mathbf{J}_{\mu}^{N}[\Psi] n_{\mathcal{I}^{+}}^{\mu} \\
& \quad+\int_{0}^{\infty} \int_{\Sigma_{\tau}}\left(\left|\tilde{Z}^{\star} \Psi\right|^{2} r^{-1-\delta}+|\Psi|^{2} r^{-3-\delta}+\zeta|T \Psi|^{2} r^{-1-\delta}+\zeta|\not W|^{2} r^{-1}\right) d \tau \\
& \leq B(\delta)\left(\int_{\Sigma_{0}} \mathbf{J}_{\mu}^{N}[\Psi] n_{\Sigma_{0}}^{\mu}+\int_{\Sigma_{0}}|\Psi|^{2}+\int_{0}^{\infty} \int_{\Sigma_{\tau}}\left[\epsilon^{-1} r^{1+\delta}|F|^{2}+\epsilon(1-\zeta)\left(|T \Psi|^{2}+|\Phi \Psi|^{2}\right)\right]\right) .
\end{aligned}
$$

If $F$ is supported in the region $\{r \leq R\}$, then one may drop the $\int_{\Sigma_{0}}|\Psi|^{2}$ term:

$$
\text { L.H.S. of (192) } \leq B(\delta, R)\left(\int_{\Sigma_{0}} \mathbf{J}_{\mu}^{N}[\Psi] n_{\Sigma_{0}}^{\mu}+\int_{0}^{\infty} \int_{\Sigma_{\tau}}\left[\epsilon^{-1} r^{1+\delta}|F|^{2}+\epsilon(1-\zeta)\left(|T \Psi|^{2}+|\Phi \Psi|^{2}\right)\right]\right) \text {. }
$$


If $F$ is supported in the region $\left\{r \geq 3 M+s_{+}\right\}$, then one may drop the $(1-\zeta)\left(|T \Psi|^{2}+|\Phi \Psi|^{2}\right)$ term:

$$
\text { L.H.S. of (192) } \leq B(\delta)\left(\int_{\Sigma_{0}} \mathbf{J}_{\mu}^{N}[\Psi] n_{\Sigma_{0}}^{\mu}+\int_{0}^{\infty} \int_{\Sigma_{\tau}} r^{1+\delta}|F|^{2}+\int_{\Sigma_{0}}|\Psi|^{2}\right) \text {. }
$$

If $F$ is supported in the region $\left\{R \geq r \geq 3 M+s_{+}\right\}$, then one may drop both:

$$
\text { L.H.S. of (192) } \leq B(\delta, R)\left(\int_{\Sigma_{0}} \mathbf{J}_{\mu}^{N}[\Psi] n_{\Sigma_{0}}^{\mu}+\int_{0}^{\infty} \int_{\Sigma_{\tau}} r^{1+\delta}|F|^{2}\right) .
$$

Proof. Repeating the proof of Proposition 9.1.1 mutatis mutandis yields

$$
\begin{aligned}
\int_{\mathcal{H}^{+}(0, \infty)} \mathbf{J}_{\mu}^{N}[\Psi] n_{\mathcal{H}^{+}}^{\mu} & +\int_{\mathcal{I}^{+}} \mathbf{J}_{\mu}^{N}[\Psi] n_{\mathcal{I}^{+}}^{\mu} \\
& +\int_{0}^{\infty} \int_{\Sigma_{\tau}}\left(\left|\tilde{Z}^{*} \Psi\right|^{2} r^{-1-\delta}+|\Psi|^{2} r^{-3-\delta}+\zeta|T \Psi|^{2} r^{-1-\delta}+\left.\zeta|\not| \Psi\right|^{2} r^{-1}\right) d \tau \\
\leq & B(\delta)\left(\int_{\Sigma_{0}} \mathbf{J}_{\mu}^{N}[\Psi] n_{\Sigma_{0}}^{\mu}+\int_{0}^{\infty} \int_{\Sigma_{\tau}}\left[\epsilon^{-1} r^{1+\delta}|F|^{2}+\epsilon(1-\zeta)\left(|T \Psi|^{2}+|\Phi \Psi|^{2}\right)\right]\right) \\
& +\int_{0}^{1} \int_{\Sigma_{s}} \mathbf{J}_{\mu}^{N}\left[\Psi \Psi_{\succ}\right] n_{\Sigma_{s}}^{\mu} d s .
\end{aligned}
$$

We cannot absorb the $\epsilon(1-\zeta)\left(|T \Psi|^{2}+|\Phi \Psi|^{2}\right)$ term into the left hand side because of the degeneration due to trapping. The final term on the right hand side arises due to the fact that $\Psi_{\odot}$ and $\Psi$ differ when $\dot{\xi} \neq 0$; since there are no weights in $r$, we cannot hope to absorb this term into the left hand side. However, an application of the fundamental theorem of calculus and Hardy inequalities easily allows us to finish the proof of (192).

In the case where $F$ is compactly supported in the region $\{r \leq R\}$, the proof of (9.1.1) yields

$$
\begin{aligned}
\int_{\mathcal{H}^{+}(0, \infty)} \mathbf{J}_{\mu}^{N}[\Psi] n_{\mathcal{H}^{+}}^{\mu} & +\int_{\mathcal{I}^{+}} \mathbf{J}_{\mu}^{N}[\Psi] n_{\mathcal{I}^{+}}^{\mu} \\
& \quad+\int_{0}^{\infty} \int_{\Sigma_{\tau}}\left(\left|\tilde{Z}^{*} \Psi\right|^{2} r^{-1-\delta}+|\Psi|^{2} r^{-3-\delta}+\zeta|T \Psi|^{2} r^{-1-\delta}+\left.\zeta|\not| \Psi\right|^{2} r^{-1}\right) d \tau \\
\leq & B(\delta)\left(\int_{\Sigma_{0}} \mathbf{J}_{\mu}^{N}[\Psi] n_{\Sigma_{0}}^{\mu}+\int_{0}^{\infty} \int_{\Sigma_{\tau}}\left[\epsilon^{-1} r^{1+\delta}|F|^{2}+\epsilon(1-\zeta)\left(|T \Psi|^{2}+|\Phi \Psi|^{2}\right)\right]\right) \\
& +\int_{0}^{1} \int_{\Sigma_{s} \cap\left[r_{+}, R\right]} \mathbf{J}_{\mu}^{N}\left[\Psi_{\odot}\right] n_{\Sigma_{s}}^{\mu} d s .
\end{aligned}
$$

The proof of (193) follows from an application of Hardy inequalities and a finite in time energy estimate to the last term on the right hand side.

The proof of (194) and (195) follow from the same reasoning as above mutatis mutandis.

Remark 9.8.1. After one has proved Theorem 3.1 it is possible to revisit the inhomogeneous problem and prove a sharper version of this proposition; however, we shall refrain from a systematic treatment of the inhomogeneous problem.

\section{The Higher ORDER STATEMENT FOR FUTURE-INTEGRABLE SOLUTIONS}

Section 9 has established the integrated decay statement (20) for the class of future-integrable solutions to the wave equation (11). Retaining this restriction, we will in this section upgrade this statement to the higher order (25). 
Proposition 10.1. Let $M>0,0 \leq a_{0}<M$. Let $|a| \leq a_{0}$ and let $\psi$ be a future integrable solution of (11) on $\mathcal{R}_{0}$. Then, for all $\delta>0$ and all integers $j \geq 1$, the following bound holds

$$
\begin{aligned}
& \int_{\mathcal{H}^{+}(0, \infty)} \sum_{1 \leq i_{1}+i_{2}+i_{3} \leq j}\left|\not^{i_{1}} T^{i_{2}}\left(\tilde{Z}^{*}\right)^{i_{3}} \psi\right|^{2}+\int_{\mathcal{I}^{+}} \sum_{1 \leq i \leq j-1} \mathbf{J}_{\mu}^{N}\left[N^{i} \psi\right] n_{\mathcal{I}^{+}}^{\mu} \\
& +\int_{\mathcal{R}_{0}} r^{-1-\delta} \zeta \sum_{1 \leq i_{1}+i_{2}+i_{3} \leq j}\left|\not^{i_{1}} T^{i_{2}}\left(\tilde{Z}^{*}\right)^{i_{3}} \psi\right|^{2} \\
& +r^{-1-\delta} \sum_{1 \leq i_{1}+i_{2}+i_{3} \leq j-1}\left(\left|\not^{i_{1}} T^{i_{2}}\left(\tilde{Z}^{*}\right)^{i_{3}+1} \psi\right|^{2}+\left|\not^{i_{1}} T^{i_{2}}\left(Z^{*}\right)^{i_{3}} \psi\right|^{2}\right) \\
& \leq B(\delta, j) \int_{\Sigma_{0}} \sum_{0 \leq i \leq j-1} \mathbf{J}_{\mu}^{N}\left[N^{i} \psi\right] n_{\Sigma_{0}}^{\mu} .
\end{aligned}
$$

10.1. Elliptic estimates. Before turning to the proof of Proposition 10.1, we will require a few standard elliptic estimates for solutions of the wave equation (11).

Throughout this section, $M>0,0 \leq a_{0}<M,|a| \leq a_{0}$ will be fixed, and $\chi$ will be a cutoff which is identically 1 on $\left[r_{+}, R_{1}\right]$ and identically 0 on $\left[R_{1}+1, \infty\right)$ for a sufficiently large constant $R_{1}$ whose $r$-value will in particular lie outside the ergoregion $\mathcal{S}$, and $Y$ will be the red-shift commutation vector field from Section 4.5 .

Lastly, for the reader's benefit we recall the following pointwise relation which follows immediately from the algebraic properties of the energy-momentum tensor:

$$
\mathbf{J}_{\mu}^{N}[\Psi] n_{\Sigma_{\tau}}^{\mu} \geq b\left((T \Psi)^{2}+\left(\tilde{Z}^{\star} \Psi\right)^{2}+\left.|\not| \Psi\right|^{2}\right) .
$$

All the lemmas below refer to solutions $\psi$ of the wave equation (11) as in the reduction of Section 4.1 .

Lemma 10.1.1. For $\psi$ as above, we have

$$
\int_{\Sigma_{\tau}} \sum_{1 \leq i_{1}+i_{2}+i_{3} \leq 2}\left|\not^{i_{1}} T^{i_{2}}\left(\tilde{Z}^{*}\right)^{i_{3}} \psi\right|^{2} \leq B \int_{\Sigma_{\tau}}\left(\mathbf{J}_{\mu}^{N}[N \psi]+\mathbf{J}_{\mu}^{N}[\psi]\right) n_{\Sigma_{\tau}}^{\mu}
$$

Proof. This is standard: Let $\hat{\Sigma}_{\tau}$ be an extension of $\Sigma_{\tau}$ from $r \in\left[r_{+}, \infty\right)$ to $r \in\left[r_{+}-\epsilon, \infty\right)$. By a standard extension lemma, one may extend $\psi$ to a function $\hat{\psi}$ on $\hat{\Sigma}_{\tau}$ in such a way that $\left\|\Delta_{\hat{\Sigma}_{\tau}} \hat{\psi}\right\|_{L^{2}\left(\hat{\Sigma}_{\tau}\right)} \leq B\left\|\Delta_{\Sigma_{\tau}} \psi\right\|_{L^{2}\left(\Sigma_{\tau}\right)}$. The lemma then follows from a local elliptic estimate.

Lemma 10.1.2. For $\psi$ as above, we have

$$
\int_{\Sigma_{\tau}} \sum_{1 \leq i_{1}+i_{2}+i_{3} \leq 2}\left|\not^{i_{1}} T^{i_{2}}\left(\tilde{Z}^{*}\right)^{i_{3}} \psi\right|^{2} \leq B \int_{\Sigma_{\tau}}\left(\mathbf{J}_{\mu}^{N}[T \psi] n_{\Sigma_{\tau}}^{\mu}+\mathbf{J}_{\mu}^{N}[\chi \Phi \psi] n_{\Sigma_{\tau}}^{\mu}+\mathbf{J}_{\mu}^{N}[Y \psi] n_{\Sigma_{\tau}}^{\mu}+\mathbf{J}_{\mu}^{N}[\psi] n_{\Sigma_{\tau}}^{\mu}\right) .
$$

Proof. This is standard: One uses elliptic estimates on spheres near the horizon and an elliptic estimate on $\Sigma_{\tau} \cap\left\{r \geq r_{0}\right\}$ away from the horizon. The key point is that $T$ and $\Phi$ span a timelike direction away from the horizon, and $Y, T$ and $\Phi$ span a timelike direction near the horizon.

Lemma 10.1.3. For $\psi$ as above, we have

$$
\begin{aligned}
& \int_{\mathcal{H}^{+}(0, \infty)} \sum_{1 \leq i_{1}+i_{2}+i_{3} \leq 2}\left|\not^{i_{1}} T^{i_{2}}\left(\tilde{Z}^{*}\right)^{i_{3}} \psi\right|^{2} \\
& \quad \leq B \int_{\mathcal{H}^{+}(0, \infty)}\left(\mathbf{J}_{\mu}^{N}[T \psi] n_{\mathcal{H}^{+}}^{\mu}+\mathbf{J}_{\mu}^{N}[\chi \Phi \psi] n_{\mathcal{H}^{+}}^{\mu}+\mathbf{J}_{\mu}^{N}[Y \psi] n_{\mathcal{H}^{+}}^{\mu}+\mathbf{J}_{\mu}^{N}[\psi] n_{\mathcal{H}^{+}}^{\mu}\right) .
\end{aligned}
$$

Proof. This follows from elliptic estimates on spheres.

One can, of course, localise Lemma 10.1.2 
Lemma 10.1.4. For $\psi$ as above, then for any $R<\infty$, we have

$$
\begin{aligned}
& \int_{\Sigma_{\tau} \cap\left[r_{+}, R\right]} \sum_{1 \leq i_{1}+i_{2}+i_{3} \leq 2}\left|\not{ }^{i_{1}} T^{i_{2}}\left(\tilde{Z}^{*}\right)^{i_{3}} \psi\right|^{2} \\
& \quad \leq B \int_{\Sigma_{\tau} \cap\left[r_{+}, R+1\right]}\left(\mathbf{J}_{\mu}^{N}[T \psi] n_{\Sigma_{\tau}}^{\mu}+\mathbf{J}_{\mu}^{N}[\chi \Phi \psi] n_{\Sigma_{\tau}}^{\mu}+\mathbf{J}_{\mu}^{N}[Y \psi] n_{\Sigma_{\tau}}^{\mu}+\mathbf{J}_{\mu}^{N}[\psi] n_{\Sigma_{\tau}}^{\mu}\right) .
\end{aligned}
$$

The next four lemmas give control of the solution without including a $Y$-commuted energy on the right hand side.

Lemma 10.1.5. For $\psi$ as above, then for any $r_{0}>r_{+}$,

$$
\int_{\Sigma_{\tau} \cap\left\{r \geq r_{0}\right\}} \mathbf{J}_{\mu}^{N}[Y \psi] n_{\Sigma_{\tau}}^{\mu} \leq B\left(r_{0}\right) \int_{\Sigma_{\tau}}\left(\mathbf{J}_{\mu}^{N}[T \psi] n_{\Sigma_{\tau}}^{\mu}+\mathbf{J}_{\mu}^{N}[\chi \Phi \psi] n_{\Sigma_{\tau}}^{\mu}+\mathbf{J}_{\mu}^{N}[\psi] n_{\Sigma_{\tau}}^{\mu}\right) .
$$

Proof. This follows from an elliptic estimate away from the horizon using the fact that the span of $T$ and $\Phi$ is timelike. The straightforward proof is omitted.

Lemma 10.1.6. For $\psi$ as above, then for any $r_{+}<r_{0}<r_{1}<\infty, \delta>0$,

$$
\int_{\Sigma_{\tau} \cap\left\{\left[r_{0}, r_{1}\right]\right\}} \mathbf{J}_{\mu}^{N}[Y \psi] n_{\Sigma_{\tau}}^{\mu} \leq B\left(r_{0}, r_{1}, \delta\right) \int_{\Sigma_{\tau} \cap\left[r_{0}-\delta, r_{1}+\delta\right]}\left(\mathbf{J}_{\mu}^{N}[T \psi] n_{\Sigma_{\tau}}^{\mu}+\mathbf{J}_{\mu}^{N}[\chi \Phi \psi] n_{\Sigma_{\tau}}^{\mu}+\mathbf{J}_{\mu}^{N}[\psi] n_{\Sigma_{\tau}}^{\mu}\right) .
$$

Proof. This straightforward proof is omitted.

Lemma 10.1.7. For $\psi$ as above, then for any, $r_{+}<r_{0}<\infty$ and $\delta_{1}, \delta_{2}>0$,

$$
\begin{aligned}
& \int_{\Sigma_{\tau} \cap\left[r_{0}, \infty\right)} \sum_{1 \leq i_{1}+i_{2}+i_{3} \leq 2} r^{-1-\delta_{1}}\left|\not^{i_{1}} T^{i_{2}}\left(\tilde{Z}^{*}\right)^{i_{3}} \psi\right|^{2} \\
& \quad \leq B\left(r_{0}, \delta_{2}\right) \int_{\Sigma_{\tau} \cap\left[r_{0}-\delta_{2}, \infty\right)} r^{-1-\delta_{1}}\left(\mathbf{J}_{\mu}^{N}[T \psi] n_{\Sigma_{\tau}}^{\mu}+\mathbf{J}_{\mu}^{N}[\chi \Phi \psi] n_{\Sigma_{\tau}}^{\mu}+\mathbf{J}_{\mu}^{N}[\psi] n_{\Sigma_{\tau}}^{\mu}\right) .
\end{aligned}
$$

Proof. This straightforward proof is omitted.

Lemma 10.1.8. For $\psi$ as above, then for any $2 M+1 \leq r_{0}<\infty$ and $\delta_{1}, \delta_{2}>0$,

$$
\int_{\Sigma_{\tau} \cap\left\{\left[r_{0}, \infty\right)\right\}} r^{-1-\delta_{1}} \mathbf{J}_{\mu}^{N}[\chi \Phi \psi] n_{\Sigma_{\tau}}^{\mu} \leq B\left(\delta_{2}\right) \int_{\Sigma_{\tau} \cap\left[r_{0}-\delta_{2}, \infty\right)}\left(r^{-1-\delta_{1}} \mathbf{J}_{\mu}^{N}[T \psi] n_{\Sigma_{\tau}}^{\mu}+r^{-1-\delta_{1}} \mathbf{J}_{\mu}^{N}[\psi] n_{\Sigma_{\tau}}^{\mu}\right) .
$$

Proof. It suffices to remark that the region $[2 M+1, \infty)$ lies outside the ergoregion (see (14)), and apply elliptic estimates as before.

The following lemma will be used in conjunction with red-shift estimate of Proposition 4.5.2 and the commutation formula for $Y$ given in Proposition 4.5.3

Lemma 10.1.9. For $\psi$ as above, then for all $\epsilon>0$, we may find a $r_{0}>r_{+}$depending on $\epsilon$ such that

$$
\int_{\Sigma_{\tau} \cap\left\{r \leq r_{0}\right\}}\left|\not{ }^{2} \psi\right|^{2} \leq B \int_{\Sigma_{\tau} \cap\left[r_{+}, r_{0}\right)}\left(\mathbf{J}_{\mu}^{N}[T \psi] n_{\Sigma_{\tau}}^{\mu}+\mathbf{J}_{\mu}^{N}[\chi \Phi \psi] n_{\Sigma_{\tau}}^{\mu}+\mathbf{J}_{\mu}^{N}[\psi] n_{\Sigma_{\tau}}^{\mu}\right)+\epsilon \int_{\Sigma_{\tau}} \mathbf{J}_{\mu}^{N}[Y \psi] n_{\Sigma_{\tau}}^{\mu} .
$$

Proof. Since $Y$ is null on $\mathcal{H}^{+}$, on $\mathcal{H}^{+}$there is no $Y^{2}$ term in the wave equation. In particular, the second derivative terms in the wave equation which contain a $Y$ derivative may be controlled by $\mathbf{J}_{\mu}^{N}[T \psi] n_{\Sigma_{\tau}}^{\mu}$ and $\mathbf{J}_{\mu}^{N}[\Phi \psi] n_{\Sigma_{\tau}}^{\mu}$. Given these observations, the lemma easily follows from elliptic estimates on spheres.

We will also need some integrated in time estimates:

Lemma 10.1.10. Let $\psi$ be as above, and let $R<\infty$. Then

$$
\int_{0}^{\infty} \int_{\Sigma_{\tau} \cap[3 M+1, R)} \mathbf{J}_{\mu}^{N}[\psi] n_{\Sigma_{\tau}}^{\mu} \leq B \int_{0}^{\infty} \int_{\Sigma_{\tau} \cap[3 M, R+1)}\left(|T \psi|^{2}+\left|\partial_{r^{*}} \psi\right|^{2}+|\psi|^{2}\right)+B \int_{\Sigma_{0}} \mathbf{J}_{\mu}^{N}[\psi] n_{\Sigma_{0}}^{\mu} .
$$


Proof. This is standard: One writes the wave equation as

$$
g^{t t} \partial_{t}^{2} \psi-\frac{4 M a r}{\rho^{2} \Delta} \partial_{t, \phi}^{2} \psi=\frac{\Delta-a^{2} \sin ^{2} \theta}{\Delta \rho^{2} \sin ^{2} \theta} \partial_{\phi}^{2} \psi-\frac{r^{2}+a^{2}}{\Delta \rho^{2}} \partial_{r^{*}}\left(\left(r^{2}+a^{2}\right) \partial_{r^{*}} \psi\right)-\frac{1}{\rho^{2} \sin \theta} \partial_{\theta}\left(\sin \theta \partial_{\theta} \psi\right),
$$

multiplies by a cutoff to $r \in[3 M, R+1)$, multiplies by $\psi$, integrates by parts, remembers the comments concerning the volume form in Section 2.2.6 and applies Hardy inequalities when appropriate.

Lemma 10.1.11. For $\psi$ as above, then for any $r_{0}>r_{+}, R<\infty$ and $\delta>0$,

$$
\begin{aligned}
\int_{0}^{\infty} & \int_{\Sigma_{\tau} \cap\left[r_{0}+\delta, R-\delta\right]} \mathbf{J}_{\mu}^{N}[\psi] n_{\Sigma_{\tau}}^{\mu} \\
& \leq B\left(r_{0}, \delta\right) \int_{0}^{\infty} \int_{\left.\Sigma_{\tau} \cap\left[r_{0}, R\right]\right)}\left(|T \psi|^{2}+\left|\partial_{r^{*}} \psi\right|^{2}+|\Phi \psi|^{2}+|\psi|^{2}\right)+B \int_{\Sigma_{0}} \mathbf{J}_{\mu}^{N}[\psi] n_{\Sigma_{0}}^{\mu} .
\end{aligned}
$$

Proof. This is proven in the same fashion as Lemma 10.1.10,

\subsection{Proof of Proposition 10.1, Now we will prove Proposition 10.1}

Proof. Let $a_{0}, M, a$ and $\psi$ be as in the statement of the proposition. Let us be given moreover $\delta>0$. We will consider the case $j=2$. The case of $j \geq 3$ follows by induction in a straightforward fashion.

First, we commute the wave equation with $T$ and obtain $\square_{g}(T \psi)=0$. Since $T \psi$ is futureintegrable, the integrated energy decay statement (20) holds for $T \psi$.

Next, we commute with $\chi \Phi$, where $\chi$ is a cutoff which is identically 1 on $\left[r_{+}, R_{1}\right]$ and identically 0 on $\left[R_{1}+1, \infty\right)$. We obtain $\square_{g}(\chi \Phi \psi)=\left(\square_{g} \chi\right) \Phi \psi+2 \nabla^{\mu} \chi \nabla_{\mu} \Phi \psi$. Now, Lemma 10.1.8 implies

$$
\begin{aligned}
\int_{0}^{\infty} \int_{\Sigma_{\tau}}\left|\square_{g}(\chi \Phi \psi)\right|^{2} & \leq B \int_{0}^{\infty} \int_{\Sigma_{\tau} \cap\left[R_{1}, \infty\right)} r^{-1-\delta}\left(\mathbf{J}_{\mu}^{N}[T \psi] n_{\Sigma_{\tau}}^{\mu}+\mathbf{J}_{\mu}^{N}[\psi] n_{\Sigma_{\tau}}^{\mu}\right) \\
& \leq B \int_{\Sigma_{0}}\left(\mathbf{J}_{\mu}^{N}[T \psi] n_{\Sigma_{0}}^{\mu}+\mathbf{J}_{\mu}^{N}[\psi] n_{\Sigma_{0}}^{\mu}\right) .
\end{aligned}
$$

In the last inequality, we used that the integrated energy decay statement holds for $T \psi$. Now, statement (195) of Proposition 9.8.1 implies that the integrated energy decay statement holds for $\chi \Phi \psi$ as long as we add $B \int_{\Sigma_{0}}\left(\mathbf{J}_{\mu}^{N}[T \psi] n_{\Sigma_{0}}^{\mu}+\mathbf{J}_{\mu}^{N}[\psi] n_{\Sigma_{0}}^{\mu}\right)$ to the right hand side of the inequality.

Finally, we turn to commutation with $Y$. We recall Proposition 4.5 .3 which implies

$$
\square_{g}(Y \Psi)=\kappa_{1} Y^{2} \Psi+\sum_{|\mathbf{m}| \leq 2, m_{4} \leq 1} c_{\mathbf{m}} E_{1}^{m_{1}} E_{2}^{m_{2}} L^{m_{3}} Y^{m_{4}} \Psi
$$

where $\kappa_{1}>0$ is proportional to the surface gravity. Next, for any $\tilde{r} \leq r_{\text {red }}$, we apply the energy estimate associated to the red-shift vector field $N$, in between the hypersurfaces $\Sigma_{0}$ and $\Sigma_{\tau}$ :

$$
\begin{aligned}
\int_{\Sigma_{\tau}} & \mathbf{J}_{\mu}^{N}[Y \psi] n_{\Sigma_{\tau}}^{\mu}+\int_{\mathcal{H}^{+}(0, \tau)} \mathbf{J}_{\mu}^{N}[Y \psi] n_{\mathcal{H}^{+}}^{\mu}+\int_{0}^{\tau} \int_{\Sigma_{s} \cap\{r \leq \tilde{r}\}} \mathbf{J}_{\mu}^{N}[Y \psi] n_{\Sigma_{s}}^{\mu} d s \\
\leq & B \int_{0}^{\tau} \int_{\Sigma_{s}}\left(1_{r \in[\tilde{r}, \tilde{r}+\delta]} \mathbf{J}_{\mu}^{N}[Y \psi] n_{\Sigma_{s}}^{\mu}+\mathcal{E}^{N}[Y \psi]\right) d s+\int_{\Sigma_{s_{1}}} \mathbf{J}_{\mu}^{N}[Y \psi] n_{\Sigma_{s_{1}}}^{\mu} .
\end{aligned}
$$

For any $\epsilon>0$, we may choose $\tilde{r}$ close enough to $r_{+}, \delta$ small enough so that $\tilde{r}+2 \delta<3 M-s^{-}$and use (198), Lemma 10.1.6, Lemma 10.1.9, Lemma 10.1.4 and the fact that $N=K+Y$, to show that

$$
\begin{aligned}
& \int_{\Sigma_{s}} \mathcal{E}^{N}[Y \psi] \\
& \leq \epsilon \int_{\Sigma_{s} \cap\{r \leq \tilde{r}\}} \mathbf{J}_{\mu}^{N}[Y \psi] n_{\Sigma_{s}}^{\mu}+B \epsilon^{-1} \int_{\Sigma_{s} \cap\left[r_{+}, \tilde{r}+2 \delta\right]}\left(\mathbf{J}_{\mu}^{N}[T \psi] n_{\Sigma_{s}}^{\mu}+\mathbf{J}_{\mu}^{N}[\chi \Phi \psi] n_{\Sigma_{s}}^{\mu}+\mathbf{J}_{\mu}^{N}[\psi] n_{\Sigma_{s}}^{\mu}\right) \\
& \leq \epsilon \int_{\Sigma_{s} \cap\{r \leq \tilde{r}\}} \mathbf{J}_{\mu}^{N}[Y \psi] n_{\Sigma_{s}}^{\mu}+B \epsilon^{-1} \int_{\Sigma_{0}}\left(\mathbf{J}_{\mu}^{N}[T \psi] n_{\Sigma_{0}}^{\mu}+\mathbf{J}_{\mu}^{N}[\chi \Phi \psi] n_{\Sigma_{0}}^{\mu}+\mathbf{J}_{\mu}^{N}[\psi] n_{\Sigma_{0}}^{\mu}\right) .
\end{aligned}
$$


Combining (200) and (199) implies

$$
\begin{gathered}
\int_{\Sigma_{\tau}} \mathbf{J}_{\mu}^{N}[Y \psi] n_{\Sigma_{\tau}}^{\mu}+\int_{\mathcal{H}^{+}(0, \tau)} \mathbf{J}_{\mu}^{N}[Y \psi] n_{\mathcal{H}^{+}}^{\mu}+\int_{0}^{\tau} \int_{\Sigma_{s} \cap\{r \leq \tilde{r}\}} \mathbf{J}_{\mu}^{N}[Y \psi] n_{\Sigma_{s}}^{\mu} d s \\
\leq B \int_{\Sigma_{0}}\left(\mathbf{J}_{\mu}^{N}[T \psi] n_{\Sigma_{0}}^{\mu}+\mathbf{J}_{\mu}^{N}[\chi \Phi \psi] n_{\Sigma_{0}}^{\mu}+\mathbf{J}_{\mu}^{N}[\psi] n_{\Sigma_{0}}^{\mu}\right) .
\end{gathered}
$$

Now, the proof concludes with applications of Lemmas 10.1.3, 10.1.2, 10.1.4, 10.1.11, 10.1.7, 10.1.1 10.1.10 and (for the null infinity $\mathcal{I}^{+}$term) straightforward $\mathbf{J}^{T}$ energy estimates in a large $r$ region.

\section{The Continuity ARgument}

In this section, we will prove

Proposition 11.1. Let $M>0$ and $|a|<M$. All solutions $\psi$ to the wave equation (1) on $\mathcal{R}_{0}$ as in the reduction of Section 4.1 (i.e. arising from smooth, compactly supported initial data on $\Sigma_{0}$ ) are future-integrable.

11.1. The reduction to fixed azimuthal frequency. We begin with the following easy but important Lemma.

Lemma 11.1.1. It suffices to prove Proposition 11.1 for solutions $\psi$ to (11) assumed moreover to be supported on an arbitrary but fixed azimuthal frequency $m$.

Proof. Let $\psi$ be a solution to the wave equation arising from smooth, compactly supported initial data, and suppose we have established Proposition 11.1 for solutions supported on any fixed azimuthal frequency. We may expand $\psi$ into its azimuthal modes: $\psi=\sum_{m \in \mathbb{Z}} \psi_{m}$. Since each $\psi_{m}$ is future-integrable, it follows by Proposition 9.1.1 that the integrated energy decay statements (20) and (25) hold for $\psi_{m}$. Orthogonality immediately implies that (20) and (25) hold for $\psi$. Finally, we simply observe that the fundamental theorem of calculus implies that

$$
\begin{aligned}
& \sup _{r \in\left[r_{+}, A\right]} \int_{0}^{\infty} \int_{\mathbb{S}^{2}} \sum_{1 \leq i_{1}+i_{2}+i_{3} \leq j}\left|\not^{i_{1}} T^{i_{2}}\left(\tilde{Z}^{*}\right)^{i_{3}} \psi\right|^{2} \sin \theta d t d \theta d \phi \\
& \leq B\left(\int_{\mathcal{H}^{+}(0, \infty)} \sum_{1 \leq i_{1}+i_{2}+i_{3} \leq j}\left|\not^{i_{1}} T^{i_{2}}\left(\tilde{Z}^{*}\right)^{i_{3}} \psi\right|^{2}+\int_{0}^{\infty} \int_{\Sigma_{s} \cap\left[r_{+}, A\right]} \sum_{1 \leq i_{1}+i_{2}+i_{3} \leq j+1}\left|\not^{i_{1}} T^{i_{2}}\left(\tilde{Z}^{*}\right)^{i_{3}} \psi\right|^{2}\right) .
\end{aligned}
$$

Lemma 11.1.1 thus implies that Proposition 11.1 follows from the following proposition:

Proposition 11.1.1. Let $M>0,|a|<M$ and $m \in \mathbb{Z}$. Let $\psi$ be a solution to the wave equation as in the reduction of Section 4.1 such that moreover, $\psi$ is supported only on the azimuthal frequency $m$. Then $\psi$ is sufficiently integrable.

The following Lemma will be very useful for the proof of Proposition 11.1.1.

Lemma 11.1.2. Let $M, a, m$, and $\psi$ be as in the statement of Proposition 11.1.1. Then, for every $\tau \geq 0$ and $\delta>0$,

$$
\begin{aligned}
& \int_{\mathcal{H}^{+}(0, \tau)} \mathbf{J}_{\mu}^{N}[\psi] n_{\mathcal{H}^{+}}^{\mu} \int_{0}^{\tau} \int_{\Sigma_{s}}\left(r^{-1}\left(1-\eta_{\left[(1+\sqrt{2}) M, 3 M+s^{+}\right]}\right)(1-3 M / r)^{2}\left(|\not \psi|^{2}+r^{-\delta}|T \psi|^{2}\right)\right. \\
&\left.+r^{-1-\delta}\left|\tilde{Z}^{*} \psi\right|^{2}+r^{-3-\delta}|\psi|^{2}\right) \\
& \leq B(\delta, m)\left(\int_{\Sigma_{0}} \mathbf{J}_{\mu}^{N}[\psi] n_{\Sigma_{0}}^{\mu}+\int_{\Sigma_{\tau}} \mathbf{J}_{\mu}^{N}[\psi] n_{\Sigma_{\tau}}^{\mu}\right) .
\end{aligned}
$$


Proof. One modifies the cutoff $\xi$ from Section 9.2 now let $\xi$ be identically 1 in between $\Sigma_{1}$ and $\Sigma_{\tau-1}$ and identically 0 to the past of $\Sigma_{0}$ and the future of $\Sigma_{\tau}$. Then, one may easily check that the arguments of Section 9 will imply the lemma. Note that we can write $\eta_{\left[(1+\sqrt{2}) M, 3 M+s^{+}\right]}$instead of $\eta_{\left[3 M-s^{-}, 3 M+s^{+}\right]}$because Lemma 6.5.1 tells us that for fixed $m$ and large $\Lambda$ the trapped set is contained in $[(1+\sqrt{2}) M, \infty)$.

Remark 11.1.1. Let us emphasise that we do not assume that $\psi$ is future-integrable. This is why we must have the term $\int_{\Sigma_{\tau}} \mathbf{J}_{\mu}^{N}[\psi] n_{\Sigma_{\tau}}^{\mu}$ on the right hand side.

Remark 11.1.2. As observed in Remark 6.5.3, we see that (for a fixed azimuthal frequency) trapping and the ergoregion are non-overlapping! This will be extremely useful in what follows.

We will also need higher order versions of Lemma 11.1.2.

Lemma 11.1.3. Let $M, a, m$, and $\psi$ be as in the statement of Proposition 11.1.1. Then, for every $\tau \geq 0, j \geq 1$ and $\delta>0$,

$$
\begin{aligned}
& \int_{\mathcal{H}^{+}(0, \tau)} \sum_{1 \leq i_{1}+i_{2}+i_{3} \leq j}\left|\not^{i_{1}} T^{i_{2}}\left(\tilde{Z}^{*}\right)^{i_{3}} \psi\right|^{2} \\
& +\int_{0}^{\tau} \int_{\Sigma_{s}} r^{-1-\delta}\left(1-\eta_{\left[(1+\sqrt{2}) M, 3 M+s^{+}\right]}\right)(1-3 M / r)^{2} \sum_{1 \leq i_{1}+i_{2}+i_{3} \leq j}\left|\not^{i_{1}} T^{i_{2}}\left(\tilde{Z}^{*}\right)^{i_{3}} \psi\right|^{2} \\
& \quad+r^{-1-\delta} \sum_{1 \leq i_{1}+i_{2}+i_{3} \leq j-1}\left(\left|\not^{i_{1}} T^{i_{2}}\left(\tilde{Z}^{*}\right)^{i_{3}+1} \psi\right|^{2}+\left|\not^{i_{1}} T^{i_{2}}\left(Z^{*}\right)^{i_{3}} \psi\right|^{2}\right) \\
& \leq B(\delta, j, m)\left(\int_{\Sigma_{0}} \sum_{0 \leq i \leq j-1} \mathbf{J}_{\mu}^{N}\left[N^{i} \psi\right] n_{\Sigma_{0}}^{\mu}+\int_{\Sigma_{\tau}} \sum_{0 \leq i \leq j-1} \mathbf{J}_{\mu}^{N}\left[N^{i} \psi\right] n_{\Sigma_{\tau}}^{\mu}\right) .
\end{aligned}
$$

Proof. This follows from repeating the arguments of Section 10.

We have the following easy corollary.

Corollary 11.1.1. Let $M, a, m$, and $\psi$ be as in the statement of Proposition 11.1.1. Then, $\psi$ is future-integrable if

$$
\sup _{\tau \geq 0} \int_{\Sigma_{\tau}} \sum_{1 \leq i_{1}+i_{2}+i_{3} \leq j}\left|\not^{i_{1}} T^{i_{2}}\left(\tilde{Z}^{*}\right)^{i_{3}} \psi\right|^{2}<\infty \quad \forall j \geq 1
$$

Proof. As in the proof of Lemma 11.1.1 we need only observe that

$$
\begin{aligned}
& \sup _{r \in\left[r_{+}, A\right]} \int_{0}^{\infty} \int_{\mathbb{S}^{2}} \sum_{1 \leq i_{1}+i_{2}+i_{3} \leq j}\left|\not^{i_{1}} T^{i_{2}}\left(\tilde{Z}^{*}\right)^{i_{3}} \psi\right|^{2} \sin \theta d t d \theta d \phi \\
& \leq B(j)\left(\int_{\mathcal{H}^{+}(0, \infty)} \sum_{1 \leq i_{1}+i_{2}+i_{3} \leq j}\left|\not^{i_{1}} T^{i_{2}}\left(\tilde{Z}^{*}\right)^{i_{3}} \psi\right|^{2}+\int_{0}^{\infty} \int_{\Sigma_{s} \cap\left[r_{+}, A\right]} \sum_{1 \leq i_{1}+i_{2}+i_{3} \leq j+1}\left|\not^{i_{1}} T^{i_{2}}\left(\tilde{Z}^{*}\right)^{i_{3}} \psi\right|^{2}\right) .
\end{aligned}
$$

The proof of Proposition 11.1.1 will be a continuity argument in the rotation parameter $a$ of the black hole. That is, fix $M>0$, and define for each $m \in \mathbb{Z}$, the set

$$
\mathcal{A}_{m}:=\left\{|a| \in[0, M): \text { the statement (202) holds for } g=g_{a, M}\right\} .
$$

We shall prove that $\mathcal{A}_{m}=[0, M)$ by showing that it is non-empty, open and closed. Proposition 11.1.1 then follows by Corollary 11.1.1.

We note first

Proposition 11.1.2. For all $m \in \mathbb{Z}$, the set $\mathcal{A}_{m}$ is non-empty.

Proof. When $a=0$, it is well known that (202) holds (even without the restriction to a fixed azimuthal frequency). One may find the (relatively short) argument in the lecture notes [27. Thus $0 \in \mathcal{A}_{m}$.

We now turn to openness. 
11.2. Openness. In this section, we will prove

Proposition 11.2.1. For all $m \in \mathbb{Z}$, the set $\mathcal{A}_{m}$ is open. That is, suppose $\stackrel{a}{\in} \in \mathcal{A}_{m}$. Then there exists $\epsilon>0$ such that $|a-\stackrel{a}{a}|<\epsilon$ implies $a \in \mathcal{A}_{m}$.

The proof proper will be given in Section 11.2 .2 below. We begin with some preliminaries.

11.2.1. Gaining derivatives. We start with a definition.

Definition 11.2.1. Let $|a|<M$ and let $\epsilon_{0}>0$ be from Lemma 4.7.2. Let $\alpha(r)$ be a function such that $V:=T+\alpha(r) \Phi$ is a smooth vector field timelike in $\mathcal{R}$ which satisfies

$$
\begin{aligned}
& V=T+\frac{a}{2 M r_{+}} \Phi, \text { when } r \in\left[r_{+}, r_{+}+\epsilon_{0} / 2\right], \\
& V=T+\frac{2 M a r}{\left(r^{2}+a^{2}\right)^{2}} \Phi, \text { when } r \in\left[r_{+}+\epsilon_{0}, \frac{M(7+\sqrt{2})}{4}\right], \\
& V=T, \text { when } r \geq \frac{M(3+\sqrt{2})}{2} .
\end{aligned}
$$

Remark 11.2.1. Note that $2 M<\frac{M(3+\sqrt{2})}{2}<M(1+\sqrt{2})$. In particular, $V$ is Killing in the region where trapping occurs in Lemmas 11.1.2 and 11.1.3.

The following Lemma can be thought of as a derivative gaining converse to Lemma 11.1.2.

Lemma 11.2.1. Let $|a| \leq a_{0}<M$, let $m \in \mathbb{Z}$, and let $\psi$ be a solution the wave equation (1) as in the reduction of Section 4.1 which is furthermore supported on the fixed azimuthal frequency $m$. Then

$$
\begin{aligned}
\int_{\Sigma_{\tau}} \mathbf{J}_{\mu}^{N}[\psi] n_{\Sigma_{\tau}}^{\mu} & \leq B(m)\left(\int_{0}^{\tau} \int_{\Sigma_{s} \cap\left\{r \leq \frac{M(3+\sqrt{2})}{2}\right\}}|\Phi \psi|^{2} d s+\int_{\Sigma_{0}} \mathbf{J}_{\mu}^{N}[\psi] n_{\Sigma_{0}}^{\mu}\right) \quad \forall \tau \geq 0 \\
& \leq B(m)\left(\int_{0}^{\tau} \int_{\Sigma_{s} \cap\left\{r \leq \frac{M(3+\sqrt{2})}{2}\right\}}|\psi|^{2} d s+\int_{\Sigma_{0}} \mathbf{J}_{\mu}^{N}[\psi] n_{\Sigma_{0}}^{\mu}\right) \quad \forall \tau \geq 0 .
\end{aligned}
$$

Proof. We apply the energy identity associated to the vector field $V$ :

$$
\int_{\Sigma_{\tau}} \mathbf{J}_{\mu}^{V}[\psi] n_{\Sigma_{\tau}}^{\mu} \leq B \int_{0}^{\tau} \int_{\Sigma_{s}}\left|\mathbf{K}^{V}[\psi]\right| d s+\int_{\Sigma_{0}} \mathbf{J}_{\mu}^{V}[\psi] n_{\Sigma_{0}}^{\mu}
$$

In view of the fact that $T$ and $\Phi$ are Killing vector fields, we have

$$
\mathbf{K}^{V}[\psi]=\mathbf{K}^{(\alpha \Phi)}[\psi]=2 \mathbf{T}(\nabla \alpha, \Phi)[\psi]=2 \frac{\Delta}{\rho^{2}} \frac{d \alpha}{d r} \mathbf{T}(Z, \Phi)[\psi]=2 \frac{\Delta}{\rho^{2}} \frac{d \alpha}{d r} \operatorname{Re}(Z \psi \overline{\Phi \psi}) .
$$

Recall that $\frac{d \alpha}{d r}$ is supported away from the horizon, so that $Z$ is a regular vector field when the expression above is non-zero. We may conclude that

$$
\left|\mathbf{K}^{V}[\psi]\right| \leq B 1_{\operatorname{supp}\left(\frac{d \alpha}{d r}\right)}\left(\epsilon\left|\partial_{r} \psi\right|^{2}+\epsilon^{-1}|\Phi \psi|^{2}\right) .
$$

Lemma 11.1.2 implies

$$
\int_{0}^{\tau} \int_{\Sigma_{s}} 1_{\operatorname{supp}\left(\frac{d \alpha}{d r}\right)}\left|\partial_{r} \psi\right|^{2} d s \leq B(m)\left(\int_{\Sigma_{\tau}} \mathbf{J}_{\mu}^{N}[\psi] n_{\Sigma_{\tau}}^{\mu}+\int_{\Sigma_{0}} \mathbf{J}_{\mu}^{N}[\psi] n_{\Sigma_{0}}^{\mu}\right) .
$$

Combining $B(m) \epsilon$ times estimate (205) with estimates (203) and (204) implies

$$
\int_{\Sigma_{\tau}} \mathbf{J}_{\mu}^{V}[\psi] n_{\Sigma_{\tau}}^{\mu} \leq B(m)\left(\epsilon^{-1} \int_{0}^{\tau} \int_{\Sigma_{s} \cap\left\{r \leq \frac{M(3+\sqrt{2})}{2}\right\}}|\Phi \psi|^{2} d s+\int_{\Sigma_{0}} \mathbf{J}_{\mu}^{N}[\psi] n_{\Sigma_{1}}^{\mu}+\epsilon \int_{\Sigma_{\tau}} \mathbf{J}_{\mu}^{N}[\psi] n_{\Sigma_{\tau}}^{\mu}\right) .
$$


In order to finish the Lemma we apply the standard red-shift argument (see the lecture notes [27]). Set

$$
A:=\sup _{0 \leq s \leq \tau} \epsilon^{-1} \int_{0}^{s} \int_{\Sigma_{s^{\prime}} \cap\left\{r \leq \frac{M(3+\sqrt{2})}{2}\right\}}|\Phi \psi|^{2} d s^{\prime}+\int_{\Sigma_{0}} \mathbf{J}_{\mu}^{N}[\psi] n_{\Sigma_{0}}^{\mu}+\epsilon \int_{\Sigma_{s}} \mathbf{J}_{\mu}^{N}[\psi] n_{\Sigma_{s}}^{\mu} .
$$

For every $0 \leq s_{1}<s_{2} \leq \tau$ and $\tilde{r}$ sufficiently close to $r_{+}$, the red-shift estimate (4.5.2) implies

$$
\begin{aligned}
& \int_{\Sigma_{s_{2}}} \mathbf{J}_{\mu}^{N}[\psi] n_{\Sigma_{s_{2}}}^{\mu}+\int_{s_{1}}^{s_{2}} \int_{\Sigma_{s} \cap\{r \leq \tilde{r}\}} \mathbf{J}_{\mu}^{N}[\psi] n_{\Sigma_{s}}^{\mu} d s \\
& \quad \leq B(m) \int_{s_{1}}^{s_{2}} \int_{\Sigma_{s} \cap\{\tilde{r} \leq r \leq \tilde{r}+1\}} \mathbf{J}_{\mu}^{N}[\psi] n_{\Sigma_{s}}^{\mu} d s+\int_{\Sigma_{s_{1}}} \mathbf{J}_{\mu}^{N}[\psi] n_{\Sigma_{s_{1}}}^{\mu} .
\end{aligned}
$$

Now, we observe that in the region $\{\tilde{r} \leq r\}$ the quantities $\mathbf{J}_{\mu}^{N}[\psi] n_{\Sigma_{s}}^{\mu}$ and $\mathbf{J}_{\mu}^{V}[\psi] n_{\Sigma_{s}}^{\mu}$ are comparable. Thus, adding $\int_{s_{1}}^{s_{2}} \int_{\Sigma_{s}} \mathbf{J}_{\mu}^{V}[\psi] n_{\Sigma_{s}}^{\mu} d s$ to both sides of (207) implies

$$
\int_{\Sigma_{s_{2}}} \mathbf{J}_{\mu}^{N}[\psi] n_{\Sigma_{s_{2}}}^{\mu}+b(m) \int_{s_{1}}^{s_{2}} \int_{\Sigma_{s}} \mathbf{J}_{\mu}^{N}[\psi] n_{\Sigma_{s}}^{\mu} d s \leq B(m) \int_{s_{1}}^{s_{2}} \int_{\Sigma_{s}} \mathbf{J}_{\mu}^{V}[\psi] n_{\Sigma_{s}}^{\mu} d s+\int_{\Sigma_{s_{1}}} \mathbf{J}_{\mu}^{N}[\psi] n_{\Sigma_{s_{1}}}^{\mu} .
$$

Now, estimate (206) (with $\tau$ on the left hand side replaced by $s$ ) implies

$$
\int_{\Sigma_{s_{2}}} \mathbf{J}_{\mu}^{N}[\psi] n_{\Sigma_{s_{2}}}^{\mu}+b(m) \int_{s_{1}}^{s_{2}} \int_{\Sigma_{s}} \mathbf{J}_{\mu}^{N}[\psi] n_{\Sigma_{s}}^{\mu} d s \leq B(m) A\left(s_{2}-s_{1}\right)+\int_{\Sigma_{s_{1}}} \mathbf{J}_{\mu}^{N}[\psi] n_{\Sigma_{s_{1}}}^{\mu} .
$$

Let

$$
f(s):=\int_{\Sigma_{s}} \mathbf{J}_{\mu}^{N}[\psi] n_{\Sigma_{s}}^{\mu}
$$

We may rewrite equation (209) as

$$
f\left(s_{2}\right)+b \int_{s_{1}}^{s_{2}} f(s) d s \leq B(m) A\left(s_{2}-s_{1}\right)+f\left(s_{1}\right) \quad \text { for every } 0 \leq s_{1}<s_{2} \leq \tau .
$$

An easy argument shows that this implies

$$
f(s) \leq B(m)(A+f(0)) .
$$

Writing this out gives

$$
\sup _{0 \leq s \leq \tau} \int_{\Sigma_{s}} \mathbf{J}_{\mu}^{N}[\psi] n_{\Sigma_{s}}^{\mu} \leq B(m)\left(\epsilon^{-1} \int_{0}^{\tau} \int_{\Sigma_{s} \cap\left\{r \leq \frac{M(3+\sqrt{2})}{2}\right\}}|\Phi \psi|^{2} d s+\int_{\Sigma_{0}} \mathbf{J}_{\mu}^{N}[\psi] n_{\Sigma_{0}}^{\mu}+\epsilon \sup _{0 \leq s \leq \tau} \int_{\Sigma_{s}} \mathbf{J}_{\mu}^{N}[\psi] n_{\Sigma_{s}}^{\mu}\right) .
$$

We conclude the proof by taking $\epsilon$ sufficiently small.

Remark 11.2.2. Observe that the proof does not exploit the fact that the ergoregion and trapping are disjoint; indeed, even without the restriction to fixed $m$, we could have proved the first line of the proposition, with a constant $B$ not depending on $m$, simply by exploiting the fact that the $\partial_{r}$ derivative does not degenerate in the integrated local energy decay. Rather, the point is that for fixed $m$, the presence of the ergoregion is only a low-frequency obstruction to boundedness.

Remark 11.2.3. Note that the proof crucially uses that we can upgrade a degenerate energy boundedness statement to a non-degenerate energy boundedness statement without a full integrated local energy decay.

Next, we play Lemmas 11.2.1 and Lemma 11.1.3 off each other. We end up being able to gain an arbitrary number of derivatives. 
Lemma 11.2.2. Let $|a| \leq a_{0}<M$, let $m \in \mathbb{Z}$ and let $\psi$ be a solution the wave equation (1) as in the reduction of Section 4.1. which is furthermore supported on the fixed azimuthal frequency $m$. Then, for every $j \geq 1$,

$$
\begin{aligned}
& \int_{\Sigma_{\tau}} \sum_{1 \leq i_{1}+i_{2}+i_{3} \leq j}\left|\not^{i_{1}} T^{i_{2}}\left(\tilde{Z}^{*}\right)^{i_{3}} \psi\right|^{2} \\
& \leq B(j, m)\left(\int_{0}^{\tau} \int_{\Sigma_{s} \cap\left\{r \leq \frac{M(3+\sqrt{2})}{2}\right\}}|\psi|^{2} d s+\int_{\Sigma_{0}} \sum_{1 \leq i_{1}+i_{2}+i_{3} \leq j}\left|\not^{i_{1}} T^{i_{2}}\left(\tilde{Z}^{*}\right)^{i_{3}} \psi\right|^{2}\right) \quad \forall \tau \geq 0 .
\end{aligned}
$$

Proof. We first consider the case $j=2$. We begin by commuting the wave equation with $T$ and applying Lemma 11.2.1. We obtain

$$
\int_{\Sigma_{\tau}} \mathbf{J}_{\mu}^{N}[T \psi] n_{\Sigma_{\tau}}^{\mu} \leq B(m)\left(\int_{0}^{\tau} \int_{\Sigma_{s} \cap\left\{r \leq \frac{M(3+\sqrt{2})}{2}\right\}}|T \psi|^{2} d s+\int_{\Sigma_{0}} \mathbf{J}_{\mu}^{N}[T \psi] n_{\Sigma_{1}}^{\mu}\right) .
$$

Now commute the wave equation with the red-shift commutation vector field $Y$. On the horizon $\mathcal{H}^{+}$ we will have

$$
\square_{g}(Y \psi)=\kappa_{1} Y^{2} \psi+\sum_{i+j+k \in[0,2], k \leq 1} c_{i j k} T^{i} \partial_{\theta}^{j} Y^{k} \psi
$$

where $\kappa_{1}>0$ is proportional to the surface gravity of $\mathcal{H}^{+}$.

Next, we apply Proposition 4.5.2, the energy estimate associated to the red-shift multiplier $N$, to $\Psi=Y \psi$. For every $1 \leq s_{1}<s_{2} \leq \tau$, we obtain

$$
\begin{aligned}
& \int_{\Sigma_{s_{2}}} \mathbf{J}_{\mu}^{N}[Y \psi] n_{\Sigma_{s_{2}}}^{\mu}+\int_{s_{1}}^{s_{2}} \int_{\Sigma_{s} \cap\{r \leq \tilde{r}\}} \mathbf{J}_{\mu}^{N}[Y \psi] n_{\Sigma_{s}}^{\mu} d s \\
& \quad \leq B \int_{s_{1}}^{s_{2}} \int_{\Sigma_{s}}\left(1_{r \in[\tilde{r}, \tilde{r}+1]} \mathbf{J}_{\mu}^{N}[Y \psi] n_{\Sigma_{s}}^{\mu}+\mathcal{E}^{N}[Y \psi]\right) d s+\int_{\Sigma_{s_{1}}} \mathbf{J}_{\mu}^{N}[Y \psi] n_{\Sigma_{s_{1}}}^{\mu} .
\end{aligned}
$$

For any $\epsilon>0$ we may choose $\tilde{r}$ close enough to $r_{+}$, and use (211), Lemma 10.1.5 Lemma 10.1.9 and the fact that $N=K+Y$, to show that

$$
\int_{\Sigma_{s}} \mathcal{E}^{N}[Y \psi] \leq \epsilon \int_{\Sigma_{s} \cap\{r \leq \tilde{r}\}} \mathbf{J}_{\mu}^{N}[Y \psi] n_{\Sigma_{s}}^{\mu}+B \epsilon^{-1} \int_{\Sigma_{s}}\left(\mathbf{J}_{\mu}^{N}[T \psi] n_{\Sigma_{s}}^{\mu}+\mathbf{J}_{\mu}^{N}[\psi] n_{\Sigma_{s}}^{\mu}\right)
$$

Adding $\int_{s_{1}}^{s_{2}} \int_{\Sigma_{s}}\left(\mathbf{J}_{\mu}^{N}[T \psi] n_{\Sigma_{s}}^{\mu}+\mathbf{J}_{\mu}^{N}[\psi] n_{\Sigma_{s}}^{\mu}\right) d s$ to both sides, using Lemma 10.1.5 and using (213) implies

$$
\begin{aligned}
& \int_{\Sigma_{s_{2}}} \mathbf{J}_{\mu}^{N}[Y \psi] n_{\Sigma_{s_{2}}}^{\mu}+b \int_{s_{1}}^{s_{2}} \int_{\Sigma_{s}} \mathbf{J}_{\mu}^{N}[Y \psi] n_{\Sigma_{s}}^{\mu} d s \\
& \quad \leq B \int_{s_{1}}^{s_{2}} \int_{\Sigma_{s}}\left(\mathbf{J}_{\mu}^{N}[T \psi] n_{\Sigma_{s}}^{\mu}+\mathbf{J}_{\mu}^{N}[\psi] n_{\Sigma_{s}}^{\mu}\right) d s+\int_{\Sigma_{s_{1}}} \mathbf{J}_{\mu}^{N}[Y \psi] n_{\Sigma_{s_{1}}}^{\mu} .
\end{aligned}
$$

Now we use (210), Lemma 11.2.1 and the same argument which occurs at the end of the proof of Lemma 11.2.1 to conclude

$$
\begin{aligned}
\int_{\Sigma_{\tau}} \mathbf{J}_{\mu}^{N}[Y \psi] n_{\Sigma_{\tau}}^{\mu} \leq & B(m) \int_{0}^{\tau} \int_{\Sigma_{s} \cap\left\{r \leq \frac{M(3+\sqrt{2})}{2}\right\}}\left(|T \psi|^{2}+|\psi|^{2}\right) d s \\
& +B(m) \int_{\Sigma_{0}}\left(\mathbf{J}_{\mu}^{N}[T \psi] n_{\Sigma_{1}}^{\mu}+\mathbf{J}_{\mu}^{N}[Y \psi] n_{\Sigma_{1}}^{\mu}+\mathbf{J}_{\mu}^{N}[\psi] n_{\Sigma_{1}}^{\mu}\right) .
\end{aligned}
$$

Next, Lemma 10.1.2 allows us to combine (215) and (210) to get

$$
\begin{aligned}
& \int_{\Sigma_{\tau}} \sum_{1 \leq i_{1}+i_{2}+i_{3} \leq 2}\left|\not^{i_{1}} T^{i_{2}}\left(\tilde{Z}^{*}\right)^{i_{3}} \psi\right|^{2} \\
& \leq B(m)\left(\int_{0}^{\tau} \int_{\Sigma_{s} \cap\left\{r \leq \frac{M(3+\sqrt{2})}{2}\right\}}\left(|T \psi|^{2}+|\psi|^{2}\right) d s+B(m) \int_{\Sigma_{0}} \sum_{1 \leq i_{1}+i_{2}+i_{3} \leq 2}\left|\not^{i_{1}} T^{i_{2}}\left(\tilde{Z}^{*}\right)^{i_{3}} \psi\right|^{2}\right) .
\end{aligned}
$$


It remains to remove the spacetime integral of $|T \psi|^{2}$ from the right hand side; however, we observe the following immediate consequence of Lemmas 11.1.2 and 11.2.1

$$
\begin{aligned}
\int_{0}^{\tau} \int_{\Sigma_{s} \cap\left\{r \leq \frac{M(3+\sqrt{2})}{2}\right\}}|T \psi|^{2} & \leq B(m)\left(\int_{\Sigma_{\tau}} \mathbf{J}_{\mu}^{N}[\psi] n_{\Sigma_{\tau}}^{\mu}+\int_{\Sigma_{0}} \mathbf{J}_{\mu}^{N}[\psi] n_{\Sigma_{0}}^{\mu}\right) \\
& \leq B(m)\left(\int_{0}^{\tau} \int_{\Sigma_{s} \cap\left\{r \leq \frac{M(3+\sqrt{2})}{2}\right\}}|\psi|^{2}+\int_{\Sigma_{0}} \mathbf{J}_{\mu}^{N}[\psi] n_{\Sigma_{0}}^{\mu}\right)
\end{aligned}
$$

Combining (216) and (217) completes the proof for $j=220$

The case for general $j \geq 2$ follows by induction in a straightforward fashion.

The following straightforward corollary will be useful in what follows.

Corollary 11.2.1. Let $|a| \leq a_{0}<M$, let $m \in \mathbb{Z}$, and let $\psi$ be a solution the wave equation (1) as in the reduction of Section 4.1 which is furthermore supported on the fixed azimuthal frequency $\mathrm{m}$. Then, for all $\delta>0, j \geq 1$,

$$
\begin{aligned}
& \sup _{\tau^{\prime} \leq \tau} \int_{\Sigma_{\tau^{\prime}}} \sum_{1 \leq i_{1}+i_{2}+i_{3} \leq j}\left|\not^{i_{1}} T^{i_{2}}\left(\tilde{Z}^{*}\right)^{i_{3}} \psi\right|^{2} \\
& +\int_{0}^{\tau} \int_{\Sigma_{\tau}}\left(\sum_{1 \leq i_{1}+i_{2}+i_{3} \leq j-1}\left|\not^{i_{1}} T^{i_{2}}\left(\tilde{Z}^{*}\right)^{i_{3}} \psi\right|^{2}+\sum_{1 \leq i_{1}+i_{2}+i_{3} \leq j-1}\left|\not^{i_{1}} T^{i_{2}}\left(\tilde{Z}^{*}\right)^{i_{3}+1} \psi\right|^{2}\right. \\
& \left.\quad+r^{-2-\delta}|\psi|^{2}+1_{\left[r_{+},(1+\sqrt{2}) M\right]} \sum_{1 \leq i_{1}+i_{2}+i_{3} \leq j}\left|\not^{i_{1}} T^{i_{2}}\left(\tilde{Z}^{*}\right)^{i_{3}} \psi\right|^{2}\right) \cdot r^{-1-\delta} \\
& \leq B(\delta, j, m)\left(\int_{0}^{\tau} \int_{\Sigma_{s} \cap\left\{r \leq \frac{M(3+\sqrt{2})}{2}\right\}}|\psi|^{2} d s+\int_{\Sigma_{0}} \sum_{1 \leq i_{1}+i_{2}+i_{3} \leq j}\left|\not^{i_{1}} T^{i_{2}}\left(\tilde{Z}^{*}\right)^{i_{3}} \psi\right|^{2}\right)
\end{aligned}
$$

Proof. This is a simple combination of Lemmas 11.2.2 and 11.1.3

11.2.2. An interpolating metric and the proof of Proposition 11.2.1. We now turn to the proof of Proposition 11.2.1.

Proof. Recall that we have fixed $M>0$. Let us suppose $m \in \mathbb{Z}$ is fixed and $\stackrel{\circ}{a} \in \mathcal{A}_{m}$. Let us choose $a_{0}$ such that $|\stackrel{a}{a}|<a_{0}<M$. We will find an $\epsilon>0$ with $|\stackrel{a}{a}|+\epsilon \leq a_{0}$ such that

$$
|a-\stackrel{a}{a}|<\epsilon
$$

implies $a \in \mathcal{A}_{m}$.

Let $a$ satisfy (218), for $\epsilon$ to be determined, and $\psi$ be a solution of the wave equation (11) on $g_{M, a}$, as in the reduction of Section 4.1, such that moreover, $\psi$ is supported on the fixed azimuthal frequency $m$.

Recall that Corollary 11.1 .1 implies that solutions $\widetilde{\psi}$ to $\square_{g_{\hat{a}, M}} \widetilde{\psi}=0$ which are supported on a fixed azimuthal frequency are known to be future-integrable. In order to exploit this "black box" knowledge about $g_{a}, M$ it is useful to introduce a metric $\tilde{g}_{\tau}$ which interpolates between $g_{a}, M$ and $g_{a, M}$. Fortunately, we will not need to fine tune $\tilde{g}_{\tau}$.

Definition 11.2.2. Pick $\tau \geq 1$. Recalling that the hypersurfaces $\Sigma_{s}$ are independent of a, let $\chi_{\tau}$ be a cut-off which is 0 in the past of $\Sigma_{\tau-\delta_{0}}$ and identically one in the future of $\Sigma_{\tau}$ for some sufficiently small $\delta_{0}>0$. We define the interpolating metric $\tilde{g}_{\tau}$ by

$$
\tilde{g}_{\tau} \doteq \chi_{\tau} g_{a}, M+\left(1-\chi_{\tau}\right) g_{a, M} .
$$

If $\epsilon$ in (218) is assumed sufficiently small, then $\tilde{g}_{\tau}$ defines a Lorentzian metric on $\mathcal{R}$.

\footnotetext{
${ }^{20}$ Observe that this final trick relies on the fact that trapping and the ergoregion are disjoint in physical space when the azimuthal frequency is fixed.
} 
Remark 11.2.4. Note that it is easy to see that $\Phi$ is a Killing vector field for the metric $\tilde{g}_{\tau}$, and that for all $\tau \geq 0, \Sigma_{\tau}$ is a past Cauchy hypersurface for $\mathcal{R}_{0} \backslash \mathcal{R}_{(0, \tau)}$ with respect to $\tilde{g}_{\tau}$.

Corresponding to our interpolating metric, we will need an "interpolating" solution to the wave equation.

Definition 11.2.3. Let $\psi$ be our solution to $\square_{g_{a, M}} \psi=0$ defined above. We define the interpolating solution $\tilde{\psi}_{\tau}$ by solving $\square_{\tilde{g}_{\tau}} \tilde{\psi}_{\tau}=0$ with the same initial data as $\psi$ on $\Sigma_{0}$.

Of course, $\tilde{\psi}_{\tau}$ will exactly equal $\psi$ in the past of $\Sigma_{\tau-\delta_{0}}$, and in the future of $\Sigma_{\tau}, \tilde{\psi}_{\tau}$ is a solution to $\square_{g_{\tilde{a}, M}} \tilde{\psi}_{\tau}=0$. Furthermore, since $\Phi$ is a Killing vector field for $\tilde{g}_{\tau}$, the interpolating solution $\tilde{\psi}_{\tau}$ will be supported on the same azimuthal frequency $m$ as the original solution $\psi$. Hence, by the assumption $\stackrel{\circ}{a} \in \mathcal{A}_{m}$ and Corollary 11.1.1, it follows that $\tilde{\psi}_{\tau}$ is future-integrable with respect to $\stackrel{\circ}{a}$.

We write

$$
\square_{g_{\hat{a}, M}} \tilde{\psi}_{\tau}=\left(\square_{g_{\hat{a}, M}}-\square_{\tilde{g}_{\tau}}\right) \tilde{\psi}_{\tau},
$$

and observe

$$
r^{1+\delta}\left|\left(\square_{g_{\tilde{a}, M}}-\square_{\tilde{g}_{\tau}}\right) \tilde{\psi}_{\tau}\right|^{2} \leq B\left(\delta_{0}^{-1}\right)|a-\stackrel{a}{ }|^{2} r^{-2} \sum_{1 \leq i_{1}+i_{2}+i_{3} \leq 2}\left|\not^{i_{1}} T^{i_{2}}\left(\tilde{Z}^{*}\right)^{i_{3}} \tilde{\psi}_{\tau}\right|^{2} .
$$

In this statement, and in what follows, metric defined quantities (such as $\not$ and $\mathbf{J}_{\mu}^{N}[\psi] n^{\mu}$ ) will refer to $g_{a}, M$. Now we apply the $g_{a}, M$ integrated local energy estimate to $\tilde{\psi}$.

Keeping in mind that (219) is supported in the past of $\Sigma_{\tau}$, Proposition 9.8.1 implies

$$
\begin{aligned}
\int_{0}^{\tau-\delta_{0}} & \int_{\Sigma_{s} \cap\{r \leq M(1+\sqrt{2})\}}\left(\mathbf{J}_{\mu}^{N}[\psi] n_{\Sigma_{s}}^{\mu}+|\psi|^{2}\right) d s \\
& \leq B\left(\delta_{0}, m\right)|a-\stackrel{\circ}{a}| \int_{0}^{\tau} \int_{\Sigma_{s}} \sum_{1 \leq i_{1}+i_{2}+i_{3} \leq 2} r^{-2}\left|\not^{i_{1}} T^{i_{2}}\left(\tilde{Z}^{*}\right)^{i_{3}} \tilde{\psi}_{\tau}\right|^{2} d s \\
& +B\left(\delta_{0}, m\right)|a-\stackrel{a}{a}| \int_{\tau}^{\infty} \int_{\Sigma_{s} \cap\left[r_{+},(1+\sqrt{2}) M\right]}\left[\left|T \tilde{\psi}_{\tau}\right|^{2}+\left|\tilde{\psi}_{\tau}\right|^{2}\right]+B(m) \int_{\Sigma_{0}}\left[\mathbf{J}_{\mu}^{N}[\psi] n_{\Sigma_{0}}^{\mu}+|\psi|^{2}\right] .
\end{aligned}
$$

For $\delta_{0}$ sufficiently small (and then fixing the value of $\delta_{0}$ ), finite in time energy estimates (and an easy domain of dependence argument) imply

$$
\int_{0}^{\tau} \int_{\Sigma_{s} \cap\left\{r \leq \frac{M(3+\sqrt{2})}{2}\right\}}\left(\mathbf{J}_{\mu}^{N}[\psi] n_{\Sigma_{s}}^{\mu}+|\psi|^{2}\right) d s \leq B \int_{0}^{\tau-\delta_{0}} \int_{\Sigma_{s} \cap\{r \leq M(1+\sqrt{2})\}}\left(\mathbf{J}_{\mu}^{N}[\psi] n_{\Sigma_{s}}^{\mu}+|\psi|^{2}\right) d s .
$$

Since $\tilde{\psi}_{\tau}$ is equal to $\psi$ along $\Sigma_{\tau-\delta_{0}}$, finite in time energy estimates for $\square_{\tilde{g}_{\tau}}$ imply

$$
\int_{\tau-\delta_{0}}^{\tau} \int_{\Sigma_{s}} \sum_{1 \leq i_{1}+i_{2}+i_{3} \leq 2} r^{-2}\left|\not \mathbb{H}^{i_{1}} T^{i_{2}}\left(\tilde{Z}^{*}\right)^{i_{3}} \tilde{\psi}_{\tau}\right|^{2} d s \leq B \int_{\Sigma_{\tau-\delta_{0}}} \sum_{1 \leq i_{1}+i_{2}+i_{3} \leq 2}\left|\not{ }^{i_{1}} T^{i_{2}}\left(\tilde{Z}^{*}\right)^{i_{3}} \psi\right|^{2} .
$$

Similarly,

$$
\left.\int_{\tau-\delta_{0}}^{\tau} \int_{\Sigma_{s}} \sum_{1 \leq i_{1}+i_{2}+i_{3} \leq 2} r^{-2}|\not|^{i_{1}} T^{i_{2}}\left(\tilde{Z}^{*}\right)^{i_{3}} \psi\right|^{2} d s \leq\left. B \int_{\Sigma_{\tau-\delta_{0}}} \sum_{1 \leq i_{1}+i_{2}+i_{3} \leq 2}|\not|^{i_{1}} T^{i_{2}}\left(\tilde{Z}^{*}\right)^{i_{3}} \psi\right|^{2} .
$$

Finally, Proposition 10.1 the fact that $\tilde{\psi}_{\tau}$ is future integrable, and finite in time energy inequalities imply

$$
\int_{\tau}^{\infty} \int_{\Sigma_{s} \cap\left[r_{+},(1+\sqrt{2}) M\right]}\left[\left|T \tilde{\psi}_{\tau}\right|^{2}+\left|\tilde{\psi}_{\tau}\right|^{2}\right] \leq B \int_{\Sigma_{\tau-\delta_{0}}} \sum_{1 \leq i_{1}+i_{2}+i_{3} \leq 2}\left|\not \dot{H}^{i_{1}} T^{i_{2}}\left(\tilde{Z}^{*}\right)^{i_{3}} \psi\right|^{2} .
$$


Combining (221), (222), (223), (224) and (225) gives

$$
\begin{aligned}
& \int_{0}^{\tau} \int_{\Sigma_{s} \cap\left\{r \leq \frac{M(3+\sqrt{2})}{2}\right\}}\left(\mathbf{J}_{\mu}^{N}[\psi] n_{\Sigma_{s}}^{\mu}+|\psi|^{2}\right) d s \\
& \leq B(m)|a-\stackrel{\circ}{a}|\left(\int_{0}^{\tau} \int_{\Sigma_{s}} \sum_{1 \leq i_{1}+i_{2}+i_{3} \leq 2} r^{-2}\left|\not^{i_{1}} T^{i_{2}}\left(\tilde{Z}^{*}\right)^{i_{3}} \psi\right|^{2} d s+\int_{\Sigma_{\tau-\delta_{0}}} \sum_{1 \leq i_{1}+i_{2}+i_{3} \leq 2}\left|\not^{i_{1}} T^{i_{2}}\left(\tilde{Z}^{*}\right)^{i_{3}} \tilde{\psi}_{\tau}\right|^{2}\right) \\
& \quad+B(m) \int_{\Sigma_{0}}\left[\mathbf{J}_{\mu}^{N}[\psi] n_{\Sigma_{0}}^{\mu}+|\psi|^{2}\right] .
\end{aligned}
$$

Now combine Corollary 11.2.1 and (226):

$$
\begin{aligned}
& \sup _{\tau^{\prime} \leq \tau} \int_{\Sigma_{\tau^{\prime}}} \sum_{1 \leq i_{1}+i_{2}+i_{3} \leq j}\left|\not^{i_{1}} T^{i_{2}}\left(\tilde{Z}^{*}\right)^{i_{3}} \psi\right|^{2} \\
& +\int_{0}^{\tau} \int_{\Sigma_{\tau}}\left(\sum_{1 \leq i_{1}+i_{2}+i_{3} \leq j-1}\left|\not^{i_{1}} T^{i_{2}}\left(\tilde{Z}^{*}\right)^{i_{3}} \psi\right|^{2}+\sum_{1 \leq i_{1}+i_{2}+i_{3} \leq j-1}\left|\not^{i_{1}} T^{i_{2}}\left(\tilde{Z}^{*}\right)^{i_{3}+1} \psi\right|^{2}\right. \\
& \left.+r^{-2-\delta}|\psi|^{2}+1_{\left[r_{+},(1+\sqrt{2}) M\right]} \sum_{1 \leq i_{1}+i_{2}+i_{3} \leq j}\left|\not^{i_{1}} T^{i_{2}}\left(\tilde{Z}^{*}\right)^{i_{3}} \psi\right|^{2}\right) r^{-1-\delta} \\
& \leq B(\delta, j, m)\left(\int_{0}^{\tau} \int_{\Sigma_{s} \cap\left\{r \leq \frac{M(3+\sqrt{2})}{2}\right\}}|\psi|^{2} d s+\int_{\Sigma_{0}} \sum_{1 \leq i_{1}+i_{2}+i_{3} \leq j}\left|\not^{i_{1}} T^{i_{2}}\left(\tilde{Z}^{*}\right)^{i_{3}} \psi\right|^{2}\right) \\
& \leq B(\delta, j, m)|a-\stackrel{\circ}{a}|\left(\int_{0}^{\tau} \int_{\Sigma_{s}} r^{-2} \sum_{1 \leq i_{1}+i_{2}+i_{3} \leq 2}\left|\not^{i_{1}} T^{i_{2}}\left(\tilde{Z}^{*}\right)^{i_{3}} \psi\right|^{2} d s+\left.\prod_{\Sigma_{\tau-\delta_{0}}} \sum_{1 \leq i_{1}+i_{2}+i_{3} \leq 2} T^{i_{2}}\left(\tilde{Z}^{*}\right)^{i_{3}} \psi\right|^{2}\right) \\
& +B(\delta, j, m) \int_{\Sigma_{0}} \sum_{0 \leq i_{1}+i_{2}+i_{3} \leq j}\left|\not^{i_{1}} T^{i_{2}}\left(\tilde{Z}^{*}\right)^{i_{3}} \psi\right|^{2} .
\end{aligned}
$$

As long as $j \geq 3$, we may take $\epsilon$ in (218) sufficiently small, absorb the $|a-\stackrel{a}{a}|$ term on the left hand side and conclude

$$
\sup _{\tau^{\prime} \leq \tau} \int_{\Sigma_{\tau^{\prime}}} \sum_{1 \leq i_{1}+i_{2}+i_{3} \leq j}\left|\not^{i_{1}} T^{i_{2}}\left(\tilde{Z}^{*}\right)^{i_{3}} \psi\right|^{2} \leq B(j, m) \int_{\Sigma_{0}} \sum_{0 \leq i_{1}+i_{2}+i_{3} \leq j}\left|\not^{i_{1}} T^{i_{2}}\left(\tilde{Z}^{*}\right)^{i_{3}} \psi\right|^{2}<\infty \quad \forall j \geq 3 .
$$

Lastly, we observe that the final inequality clearly remains true if we define $\not^{i_{1}}$ with respect to $g_{a, M}$ instead of $g_{a}, M$.

11.3. Closedness. To finish the proof, it remains to show

Proposition 11.3.1. The set $\mathcal{A}_{m}$ is closed in $[0, M)$. That is, suppose we have a sequence $\left\{a_{k}\right\}_{k=1}^{\infty}$ with $a_{k} \in \mathcal{A}_{m}$ and $a_{k} \rightarrow a \in(-M, M)$. Then $a \in \mathcal{A}_{m}$.

Proof. Let $\psi$ be a solution the wave equation $\square_{g_{a, M}} \psi=0$ arising from smooth, compactly supported initial data which is furthermore supported on a fixed azimuthal frequency $m$.

We may choose $a_{0}<M$ such that $|a|<a_{0}$ and without loss of generality, we may assume that $\left|a_{k}\right| \leq a_{0}$ for all $k$.

We define a sequence of functions $\psi_{k}$ by solving $\square_{g_{a_{k}, M}} \psi_{k}=0$ with the same initial data as $\psi$. Using the future-integrability of $\psi_{k}$, for every $j \geq 1$ we will have

$$
\begin{aligned}
& \int_{0}^{\infty} \int_{\Sigma_{s}} r^{-1-\delta}\left(1-\eta_{\left[(1+\sqrt{2}) M, 3 M+s^{+}\right]}\right)(1-3 M / r)^{2} \sum_{1 \leq i_{1}+i_{2}+i_{3} \leq j}\left|\not^{i_{1}} T^{i_{2}}\left(\tilde{Z}^{*}\right)^{i_{3}} \psi_{k}\right|^{2} \\
& +r^{-1-\delta} \sum_{1 \leq i_{1}+i_{2}+i_{3} \leq j-1}\left(\left|\not^{i_{1}} T^{i_{2}}\left(\tilde{Z}^{*}\right)^{i_{3}+1} \psi_{k}\right|^{2}+\left|\not^{i_{1}} T^{i_{2}}\left(Z^{*}\right)^{i_{3}} \psi_{k}\right|^{2}\right) \\
& \leq B(\delta, j, m) \int_{\Sigma_{0}} \sum_{0 \leq i \leq j-1} \mathbf{J}_{\mu}^{N}\left[N^{i} \psi_{k}\right] n_{\Sigma_{0}}^{\mu} .
\end{aligned}
$$


Now, using the fact that the region $\{r \leq M(1+\sqrt{2})\}$ contains the ergoregion $\mathcal{S}$, combining (227) and an $N$-based energy estimate yields

$$
\begin{aligned}
\sup _{\tau \geq 0} \int_{\Sigma_{\tau}} \mathbf{J}_{\mu}^{N}\left[\psi_{k}\right] n_{\Sigma_{\tau}}^{\mu} & \leq B(m) \int_{\Sigma_{0}} \mathbf{J}_{\mu}^{N}\left[\psi_{k}\right] n_{\Sigma_{0}}^{\mu}+B(m) \int_{0}^{\infty} \int_{\Sigma_{s} \cap\{r \leq M(1+\sqrt{2})\}} \mathbf{J}_{\mu}^{N}\left[\psi_{k}\right] n_{\Sigma_{s}}^{\mu} \\
& \leq B(m) \int_{\Sigma_{0}} \mathbf{J}_{\mu}^{N}\left[\psi_{k}\right] n_{\Sigma_{0}}^{\mu} .
\end{aligned}
$$

It remains to upgrade (228) to its higher order version in the (by now) standard fashion. First we commute with $T$ and obtain

$$
\sup _{\tau \geq 0} \int_{\Sigma_{\tau}} \mathbf{J}_{\mu}^{N}\left[T \psi_{k}\right] n_{\Sigma_{\tau}}^{\mu} \leq B(m) \int_{\Sigma_{0}} \mathbf{J}_{\mu}^{N}\left[T \psi_{k}\right] n_{\Sigma_{0}}^{\mu}
$$

Next, we commute with $Y$ and follow the same argument as in the proof of Lemma 11.2.2 We obtain

$$
\sup _{\tau \geq 0} \int_{\Sigma_{\tau}} \mathbf{J}_{\mu}^{N}\left[Y \psi_{k}\right] n_{\Sigma_{\tau}}^{\mu} \leq B(m) \int_{\Sigma_{0}}\left(\mathbf{J}_{\mu}^{N}\left[\psi_{k}\right] n_{\Sigma_{0}}^{\mu}+\mathbf{J}_{\mu}^{N}\left[T \psi_{k}\right] n_{\Sigma_{0}}^{\mu}+\mathbf{J}_{\mu}^{N}\left[Y \psi_{k}\right] n_{\Sigma_{0}}^{\mu}\right) .
$$

Just as in the proof of Lemma 11.2.2, elliptic estimates imply

$$
\sup _{\tau \geq 0} \int_{\Sigma_{\tau}} \sum_{1 \leq i_{1}+i_{2}+i_{3} \leq 2}\left|\not{ }^{i_{1}} T^{i_{2}}\left(\tilde{Z}^{*}\right)^{i_{3}} \psi_{k}\right|^{2} \leq B(m) \int_{\Sigma_{0}}\left(\mathbf{J}_{\mu}^{N}\left[\psi_{k}\right] n_{\Sigma_{0}}^{\mu}+\mathbf{J}_{\mu}^{N}\left[T \psi_{k}\right] n_{\Sigma_{0}}^{\mu}+\mathbf{J}_{\mu}^{N}\left[Y \psi_{k}\right] n_{\Sigma_{0}}^{\mu}\right) .
$$

Finally, an easy induction argument will imply

$$
\left.\sup _{\tau \geq 0} \int_{\Sigma_{\tau}} \sum_{1 \leq i_{1}+i_{2}+i_{3} \leq j}|\not|^{i_{1}} T^{i_{2}}\left(\tilde{Z}^{*}\right)^{i_{3}} \psi_{k}\right|^{2} \leq B(j, m) \int_{\Sigma_{0}} \sum_{1 \leq i_{1}+i_{2}+i_{3} \leq j}\left|\not^{i_{1}} T^{i_{2}}\left(\tilde{Z}^{*}\right)^{i_{3}} \psi_{k}\right|^{2} .
$$

Then, we conclude the proof by observing

$$
\begin{aligned}
\int_{\Sigma_{\tau}} \sum_{1 \leq i_{1}+i_{2}+i_{3} \leq j}\left|\not^{i_{1}} T^{i_{2}}\left(\tilde{Z}^{*}\right)^{i_{3}} \psi\right|^{2} & =\lim _{k \rightarrow \infty} \int_{\Sigma_{\tau}} \sum_{1 \leq i_{1}+i_{2}+i_{3} \leq j}\left|\not^{i_{1}} T^{i_{2}}\left(\tilde{Z}^{*}\right)^{i_{3}} \psi_{k}\right|^{2} \\
& \leq B(j, m) \int_{\Sigma_{0}} \sum_{1 \leq i_{1}+i_{2}+i_{3} \leq j}\left|\not^{i_{1}} T^{i_{2}}\left(\tilde{Z}^{*}\right)^{i_{3}} \psi\right|^{2} .
\end{aligned}
$$

The first equality uses the well-posedness of the wave equation, the smooth dependence of $g_{a, M}$ on $a$ (see Lemma 4.1.1) and the fact that $\psi_{k}$ and $\psi$ have the same initial data along $\Sigma_{0}$.

\section{The PRecise integrated LoCAl EnERGy DeCAy Statement}

In this section we give will a more precise form of the integrated local energy decay statement. So as to produce a purely physical space estimate, we employed in the proof of Proposition 9.1.1 a physical space cutoff $\zeta$ (see (13)) in the integrated energy decay statement (20). It is clear from the statement of Theorem 8.1 in Section 8 that this throws away information (cf. the discussion in Section 8.91).

In order to succinctly state the microlocally precise form of integrated local energy decay, we introduce the following notation: For a sufficiently integrable function $\Psi$ on $\mathcal{R}$, we define

$$
\mathcal{P}_{\text {trap }}[\Psi] \doteq \frac{1}{\sqrt{2 \pi}} \int_{-\infty}^{\infty} \sum_{m \ell}\left|\zeta-(1-\zeta) r^{-1} r_{\text {trap }}\right| e^{-i \omega t} \Psi_{m \ell}^{(a \omega)}(r) S_{m \ell}(a \omega, \cos \theta) e^{i m \phi} d \omega
$$

where $r_{\text {trap }}=r_{\text {trap }}\left(\omega, m, \Lambda_{m \ell}\right)$ is defined in Theorem 8.1. Then, we have

Proposition 12.1. Let $0 \leq a_{0}<M, 0 \leq a \leq a_{0}$, and let $\psi$ be a solution of (1) on $\mathcal{R}_{0}$ as in the reduction of Section 9.1.1. Then,

$b \int_{0}^{\infty} \int_{\Sigma_{\tau}}\left(\left|\tilde{Z}^{*} \psi\right|^{2} r^{-1-\delta}+|\psi|^{2} r^{-3-\delta}+\left|T \mathcal{P}_{\text {trap }}[\xi \psi]\right|^{2} r^{-1-\delta}+\left|\not \mathcal{P}_{\text {trap }}[\xi \psi]\right|^{2} r^{-1}\right) d r^{*} d \omega \leq B \int_{\Sigma_{0}} \mathbf{J}_{\mu}^{N}[\psi] n_{\Sigma_{0}}^{\mu}$.

where $r_{\text {trap }}=r_{\text {trap }}\left(\omega, m, \Lambda_{m \ell}\right)$ is defined in Theorem 8.1 and $\xi$ is the cutoff from Section $\square$. 
Proof. One revisits the proof of Proposition 9.1.1 and simply retains the nonnegative term on the left hand side of (232) instead of applying the physical space $\zeta$ and the inequality (165) .

\section{ENERGY BOUNDEDNESS}

In this section, we establish the uniform boundedness of the energy flux through $\Sigma_{\tau}$ for solutions $\psi$ to the wave equation (1):

Proposition 13.1. Let $0 \leq a_{0}<M,|a| \leq a_{0}$ and let $\psi$ be a solution of the wave equation (11) on $\mathcal{R}_{0}$ as in the reduction of Section 4.1. Then

$$
\int_{\Sigma_{\tau}} \mathbf{J}_{\mu}^{N}[\psi] n_{\Sigma_{\tau}}^{\mu} \leq B \int_{\Sigma_{0}} \mathbf{J}_{\mu}^{N}[\psi] n_{\Sigma_{0}}^{\mu} \quad \forall \tau \geq 0
$$

First, recall that the arguments of Sections 9 and 11 have shown that $\psi$ is future-integrable and satisfies the integrated decay statements (20) and (25).

Let $\delta>0$ be a fixed small parameter, $A_{0}$ be sufficiently close to $r_{+}$and $A_{1}$ be sufficiently large. The proof proceeds in three steps where the cases $r \in\left[A_{0}+\delta, A_{1}-\delta\right], r \in\left[r_{+}, A_{0}+\delta\right]$ and $r \in\left[A_{1}-\delta, \infty\right)$ are each dealt with. As one expects, the first region is the most difficult.

13.1. Boundedness of $\int_{\Sigma_{\tau} \cap\left[A_{0}+\delta, A_{1}-\delta\right]} \mathbf{J}_{\mu}^{N}[\psi] n_{\Sigma_{\tau}}^{\mu}$. It turns out to be convenient to extend the solution to the entire domain of outer communication $\mathcal{R}$ from $\mathcal{R}_{0}$.

13.1.1. Extending the solution. The trace of $\psi$ and $N \psi$ along the hypersurface $\Sigma_{0}$ only suffice to determine $\psi$ in the future of $\Sigma_{0}$. However, an easy domain of dependence argument and finite in time energy estimates allow one to extend $\psi$ to the $\mathcal{R}$ in such a way as to guarantee

$$
\int_{\hat{\Sigma}_{0}} \mathbf{J}_{\mu}^{N}[\psi] n_{\Sigma_{\tau}}^{\mu} \leq B \int_{\Sigma_{0}} \mathbf{J}_{\mu}^{N}[\psi] n_{\Sigma_{0}}^{\mu} .
$$

Here $\hat{\Sigma}_{0}$ denotes the image of $\Sigma_{0}$ under the Boyer-Lindquist coordinate defined map: $t \mapsto-t$.

13.1.2. Integrated local energy decay for the extended solution. Since the Boyer-Lindquist defined map $t \mapsto-t$ and $a \mapsto-a$ is an isometry, Proposition 9.1.1 remains true if one goes to the past instead of the future, i.e. if we replace all integrals $\int_{0}^{\infty}$ with $\int_{-\infty}^{0}$, and replace $\Sigma_{\tau}$ with $\hat{\Sigma}_{\tau}$. Keeping (233) in mind, we conclude

$$
\int_{-\infty}^{\infty} \int_{\Sigma_{\tau} \cap\left[A_{0}, A_{1}\right]}\left(\left|\partial_{r^{*}} \psi\right|^{2}+r^{-2}|\psi|^{2}+\zeta \mathbf{J}_{\mu}^{N}[\psi] n_{\Sigma_{\tau}}^{\mu}\right) r^{-1-\delta} d \tau \leq B\left(A_{0}, A_{1}\right) \int_{\Sigma_{0}} \mathbf{J}_{\mu}^{N}[\psi] n_{\Sigma_{0}}^{\mu} .
$$

Unfortunately, this version of integrated local energy decay is too crude for our purposes, and we shall need to appeal to the version (232) of integrated local energy decay.

Let $\chi_{\left[A_{0}, A_{1}\right]}$ be a bump function which is identically 1 when $r \in\left[A_{0}+\delta, A_{1}-\delta\right]$ and 0 when $r \notin\left[A_{0}, A_{1}\right]$. We define

We will have

$$
\tilde{\psi} \doteq \chi_{\left[A_{0}, A_{1}\right]} \psi \text {. }
$$

$$
\square_{g_{a, M}} \tilde{\psi}=\tilde{F} \doteq \square_{g} \chi_{\left[A_{0}, A_{1}\right]} \psi+2 \nabla^{\mu} \chi_{\left[A_{0}, A_{1}\right]} \nabla_{\mu} \psi .
$$

Observe that $\tilde{F}$ has compact support in $r$, and $|\tilde{F}|^{2} \leq B\left(|\psi|^{2}+\left|\partial_{r^{*}} \psi\right|^{2}\right)$. In particular, $\tilde{\psi}$ is sufficiently integrable in the sense of Definition 5.1.1 and outgoing in the sense of Definition 5.1.2. We also have

$$
\int_{-\infty}^{\infty} \int_{\Sigma_{\tau}}|\tilde{F}|^{2} \leq B \int_{-\infty}^{\infty} \int_{\Sigma_{\tau} \cap\left\{\left[A_{0}, A_{0}+\delta\right] \cup\left[A_{1}-\delta, A_{1}\right]\right\}}\left(|\psi|^{2}+\left|\partial_{r^{*}} \psi\right|^{2}\right) \leq B \int_{\Sigma_{0}} \mathbf{J}_{\mu}^{N}[\psi] n_{\Sigma_{0}}^{\mu} .
$$

Now we apply Carter's separation as defined in Section 5 and obtain

$$
\tilde{u}^{\prime \prime}+\left(\omega^{2}-V\right) \tilde{u}=\tilde{H}
$$

Note that the compact $r$ support in $\left[A_{0}, A_{1}\right]$ of $\tilde{\psi}$ is inherited by $\tilde{u}$. We apply now Theorem 8.1. In view of the support of $\tilde{u}$, it follows that the term $|\tilde{u}(-\infty)|^{2}$ vanishes. Furthermore, the right hand sides of all the frequency localised multiplier estimates (69) are $O(\tilde{H})$, and hence are supported in 
$\left[A_{0}, A_{0}+\delta\right] \cup\left[A_{1}-\delta, A_{1}\right]$. Consequently, we can apply the (now trivial) arguments of Section 9 to conclude the inhomogeneous version of (232)

$$
\begin{aligned}
b \int_{-\infty}^{\infty} \int_{\Sigma_{\tau}}\left(\left|\partial_{r^{*}} \tilde{\psi}\right|^{2}+|\tilde{\psi}|^{2}+\mathbf{J}_{\mu}^{N}\left[\mathcal{P}_{\operatorname{trap}} \tilde{\psi}\right] n_{\Sigma_{\tau}}^{\mu}\right) & \leq \int_{-\infty}^{\infty} \int_{\Sigma_{\tau} \cap\left\{\left[A_{0}, A_{0}+\delta\right] \cup\left[A_{1}-\delta, A_{1}\right]\right\}}\left(|\psi|^{2}+|\partial \psi|^{2}\right) \\
& \leq B \int_{\Sigma_{0}} \mathbf{J}_{\mu}^{N}[\psi] n_{\Sigma_{0}}^{\mu},
\end{aligned}
$$

where we recall that $\mathcal{P}_{\text {trap }}$ is defined by (231).

13.1.3. A decomposition. In order to work around the presence of $\mathcal{P}_{\text {trap }}$ in (236), it will be useful to decompose $\tilde{\psi}$ is pieces, each of which experience trapping near a specific value of $r$. Recalling the definition of $r_{\text {trap }}$ from Theorem 8.1 we make the following definition.

Definition 13.1.1. Let $\epsilon>0$ be a sufficiently small parameter to fixed later. We define

$$
\begin{gathered}
\mathcal{C}_{0}:=\left\{(\omega, m, \Lambda): r_{\text {trap }}=0\right\}, \\
\mathcal{C}_{i}:=\left\{(\omega, m, \Lambda): r_{\text {trap }} \in\left[3 M-s^{-}+(i-1) \epsilon, 3 M-s^{-}+i \epsilon\right)\right\} \forall i=1, \ldots,\left[\epsilon^{-1}\left(s^{+}+s^{-}\right)\right] .
\end{gathered}
$$

Observe that each value of $(\omega, m, \Lambda)$ lies in exactly one of the $\mathcal{C}_{i}$.

Definition 13.1.2. We define $\tilde{\psi}_{i}$ by a phase space multiplication of $\tilde{\psi}$ by $1_{\mathcal{C}_{i}}$, the indicator function of $\mathcal{C}_{i}$ :

$$
\tilde{\psi}_{i} \doteq \frac{1}{\sqrt{2 \pi}} \int_{-\infty}^{\infty} \sum_{m \ell} e^{-i \omega t} 1_{\mathcal{C}_{i}} \tilde{\psi}_{m \ell}^{(a \omega)}(r) S_{m \ell}(a \omega, \cos \theta) e^{i m \phi} d \omega .
$$

Note that it immediately follows from Plancherel (see Section 5.2.2) that each $\tilde{\psi}_{i}$ is sufficiently integrable, and we have $\square_{g_{a, M}} \tilde{\psi}_{i}=\tilde{F}_{i}$ where $\tilde{F}_{i}$ is defined in the same fashion as $\tilde{\psi}_{i}$.

It will be useful to observe the following.

Proposition 13.1.1. For each $i=0, \ldots,\left[\epsilon^{-1}\left(s^{+}+s^{-}\right)\right]$there exists a constant $C_{i}$ and a dyadic sequence $\left\{\tau_{n}^{(i)}\right\}_{n=1}^{\infty}$ such that $\tau_{n}^{(i)} \rightarrow-\infty$ as $n \rightarrow \infty$ and

$$
\int_{\Sigma_{\tau_{n}(i)}} \mathbf{J}_{\mu}^{N}\left[\tilde{\psi}_{i}\right] n_{\Sigma_{\tau_{n}^{(i)}}^{\mu}}^{\mu} \leq \frac{C_{i}}{\tau_{n}^{(i)}}
$$

Proof. Since each $\tilde{\psi}_{i}$ is sufficiently integrable and compactly supported in $r$, we have

$$
\int_{-\infty}^{\infty} \int_{\Sigma_{\tau}} \mathbf{J}_{\mu}^{N}\left[\tilde{\psi}_{i}\right] n_{\Sigma_{\tau}}^{\mu}<\infty
$$

The proof then concludes with a standard pigeonhole argument.

13.1.4. Boundedness. Finally, we will establish boundedness of the energy of $\tilde{\psi}$.

Proposition 13.1.2. Under the assumptions of Proposition 13.1 and with the above notation we have

$$
\int_{\Sigma_{\tau}} \mathbf{J}_{\mu}^{N}[\tilde{\psi}] n_{\Sigma_{\tau}}^{\mu} \leq B \int_{\Sigma_{0}} \mathbf{J}_{\mu}^{N}[\psi] n_{\Sigma_{0}}^{\mu} \quad \forall \tau \in(-\infty, \infty)
$$

Proof. Since $\tilde{\psi}=\sum_{i=0}^{\left[\epsilon^{-1}\left(s_{+}+s_{-}\right)\right]} \tilde{\psi}_{i}$, it suffices to prove the proposition with $\tilde{\psi}$ replaced by $\tilde{\psi}_{i}$.

In Proposition 4.7.1 we showed that the vector field $T+\frac{2 M a r}{\left(r^{2}+a^{2}\right)^{2}} \Phi$ is timelike in $\mathcal{R} \backslash \mathcal{H}^{+}$. Given this and taking $\epsilon$ from Definition 13.1.1 sufficiently small (and then fixing $\epsilon$ ), it is easy to construct a $\varphi_{\tau}$-invariant timelike vector field $V_{i}$ on $\mathcal{R}$ which is Killing in the region

$$
r \in\left[3 M-s^{-}+(i-1) \epsilon, 3 M-s^{-}+i \epsilon\right) .
$$


Now we apply the energy identity associated to $V_{i}$ in between the hypersurfaces $\Sigma_{\tau}$ and $\Sigma_{\tau_{n}^{(i)}}$. Since

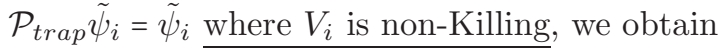

$$
\begin{aligned}
\int_{\Sigma_{\tau}} \mathbf{J}_{\mu}^{V_{i}}\left[\tilde{\psi}_{i}\right] n_{\Sigma_{\tau}}^{\mu} & \leq B \int_{\tau_{n}^{(i)}}^{\tau} \int_{\Sigma_{s} \cap\left[3 M-s^{-}+(i-1) \epsilon, 3 M-s^{-}+i \epsilon\right] c} \mathbf{J}_{\mu}^{N}\left[\tilde{\psi}_{i}\right] n_{\Sigma_{s}}^{\mu}+\int_{\Sigma_{\tau_{n}}^{(i)}} \mathbf{J}_{\mu}^{V_{i}}\left[\tilde{\psi}_{i}\right] n_{\Sigma_{\tau}}^{\mu} \\
& \leq B \int_{-\infty}^{\infty} \int_{\Sigma_{s} \cap\left[3 M-s^{-}+(i-1) \epsilon, 3 M-s^{-}+i \epsilon\right]^{c}} \mathbf{J}_{\mu}^{N}\left[\mathcal{P}_{\operatorname{trap}} \tilde{\psi}_{i}\right] n_{\Sigma_{s}}^{\mu}+\frac{B C_{i}}{\tau_{n}^{(i)}} \\
& \leq B \int_{-\infty}^{\infty} \int_{\Sigma_{s}} \mathbf{J}_{\mu}^{N}\left[\mathcal{P}_{\text {trap }} \tilde{\psi}\right] n_{\Sigma_{s}}^{\mu}+\frac{B C_{i}}{\tau_{n}^{(i)}} \\
& \leq B \int_{\Sigma_{0}} \mathbf{J}_{\mu}^{N}[\psi] n_{\Sigma_{0}}^{\mu}+\frac{B C_{i}}{\tau_{n}^{(i)}},
\end{aligned}
$$

where we have used (236) as well as Plancherel. It remains to take $n \rightarrow \infty$ and to observe (the trivial fact) that, in view of the support of $\tilde{\psi}_{i}$ and the $\phi_{\tau}$-invariance of $V_{i}$ we have $\mathbf{J}_{\mu}^{V_{i}}\left[\tilde{\psi}_{i}\right] n_{\Sigma_{\tau}}^{\mu} \sim$ $\mathbf{J}_{\mu}^{N}\left[\tilde{\psi}_{i}\right] n_{\Sigma_{\tau}}^{\mu}$.

13.2. Boundedness of $\int_{\Sigma_{\tau} \cap\left[r_{+}, A_{0}+\delta\right]} \mathbf{J}_{\mu}^{N}[\psi] n_{\Sigma_{\tau}}^{\mu}$ and $\int_{\Sigma_{\tau} \cap\left[A_{1}-\delta, \infty\right)} \mathbf{J}_{\mu}^{N}[\psi] n_{\Sigma_{\tau}}^{\mu}$. The following is a trivial consequence of the red-shift estimate (Proposition 4.5.2) and Proposition 9.1.1.

$$
\begin{aligned}
\int_{\Sigma_{\tau} \cap\left[r_{+}, A_{0}+\delta\right)} \mathbf{J}_{\mu}^{N}[\psi] n_{\Sigma_{\tau}}^{\mu} & \leq \int_{\Sigma_{0}} \mathbf{J}_{\mu}^{N}[\psi] n_{\Sigma_{0}}^{\mu}+B \int_{0}^{\tau} \int_{\Sigma_{s} \cap\left[A_{0}+\delta, A_{0}+2 \delta\right]} \mathbf{J}_{\mu}^{N}[\psi] n_{\Sigma_{s}}^{\mu} \\
& \leq B \int_{\Sigma_{0}} \mathbf{J}_{\mu}^{N}[\psi] n_{\Sigma_{0}}^{\mu} .
\end{aligned}
$$

Similarly, we may consider the energy estimate associated to $\chi_{A_{1}-\delta} T$ where $\chi_{A_{1}-\delta}$ is a cutoff which is identically 1 on $\left[A_{1}-\delta, \infty\right)$ and identically 0 on $\left[r_{+}, A_{1}-2 \delta\right]$. We obtain

$$
\begin{aligned}
\int_{\Sigma_{\tau} \cap\left[A_{1}-\delta, \infty\right)} \mathbf{J}_{\mu}^{T}[\psi] n_{\Sigma_{\tau}}^{\mu} & \leq \int_{\Sigma_{0}} \mathbf{J}_{\mu}^{N}[\psi] n_{\Sigma_{0}}^{\mu}+B \int_{0}^{\tau} \int_{\Sigma_{s} \cap\left[A_{1}-2 \delta, A_{1}-\delta\right]} \mathbf{J}_{\mu}^{N}[\psi] n_{\Sigma_{s}}^{\mu} \\
& \leq B \int_{\Sigma_{0}} \mathbf{J}_{\mu}^{N}[\psi] n_{\Sigma_{0}}^{\mu} .
\end{aligned}
$$

13.3. Putting everything together and the higher order statement. Combining Proposition 13.1.2, (238) and (239) concludes the proof of Proposition 13.1, In view of Section 3.4, this completes the proof of Theorem 3.1 .

For Theorem 3.2, we are left only with proving the higher order version of Proposition 13.1.

Proposition 13.3.1. With the notation of Proposition 13.1, for every $j \geq 1$

$$
\int_{\Sigma_{\tau}} \sum_{0 \leq i \leq j-1} \mathbf{J}_{\mu}^{N}\left[N^{i} \psi\right] n_{\Sigma_{\tau}}^{\mu} \leq B(j) \int_{\Sigma_{0}} \sum_{0 \leq i \leq j-1} \mathbf{J}_{\mu}^{N}\left[N^{i} \psi\right] n_{\Sigma_{0}}^{\mu}, \quad \forall \tau \geq 0 .
$$

Proof. We will be brief, since we have already seen multiple times how to upgrade lower order statements to higher order ones. As usual, we will only consider the case $j=2$ as the general case will follow by an easy induction argument.

First we commute (10) with $T$ and apply Proposition 13.1 We obtain

$$
\int_{\Sigma_{\tau}} \mathbf{J}_{\mu}^{N}[T \psi] n_{\Sigma_{\tau}}^{\mu} \leq B(j) \int_{\Sigma_{0}} \mathbf{J}_{\mu}^{N}[T \psi] n_{\Sigma_{0}}^{\mu}, \quad \forall \tau \geq 0
$$

Next, letting $\chi$ be a cutoff which vanishes for large $r$, we commute with $\chi \Phi$. Using the integrated energy decay to the handle resulting error terms, we obtain

$$
\int_{\Sigma_{\tau}} \mathbf{J}_{\mu}^{N}[\chi \Phi \psi] n_{\Sigma_{\tau}}^{\mu} \leq B(j) \int_{\Sigma_{0}}\left(\mathbf{J}_{\mu}^{N}[N \psi] n_{\Sigma_{0}}^{\mu}+\mathbf{J}_{\mu}^{N}[\psi] n_{\Sigma_{0}}^{\mu}\right), \quad \forall \tau \geq 0 .
$$


Finally, we commute with the red-shift commutation vector field $Y$ and apply the argument from the proofs of Lemma 11.2.2 and Proposition 10.1 to establish

$$
\int_{\Sigma_{\tau}} \mathbf{J}_{\mu}^{N}[Y \psi] n_{\Sigma_{\tau}}^{\mu} \leq B(j) \int_{\Sigma_{0}}\left(\mathbf{J}_{\mu}^{N}[N \psi] n_{\Sigma_{0}}^{\mu}+\mathbf{J}_{\mu}^{N}[\psi] n_{\Sigma_{0}}^{\mu}\right), \quad \forall \tau \geq 0
$$

The proof concludes via standard elliptic estimates (see the proofs of Lemma 11.2 .2 and Proposition 10.1).

In view of Section 3.4, this obtains the remaining statement (28) of Theorem 3.2. The proof of both main theorems is thus complete.

\section{REFERENCES}

[1] S. Alexakis, A. Ionescu and S. Klainerman Uniqueness of smooth stationary black holes in vacuum: small perturbations of the Kerr spaces, Comm. Math. Phys. 299 (2010), no. 1, 89-127.

[2] S. Alinhac Energy multipliers for perturbations of Schwarzschild metric Comm. Math. Phys. 288 (2009), no. 1, 199-224.

[3] L. Andersson and P. Blue Hidden symmetries and decay for the wave equation on the Kerr spacetime, arXiv:0908.2265.

[4] L. Andersson and P. Blue Uniform energy bound and asymptotics for the Maxwell field on a slowly rotating Kerr black hole exterior, arXiv:13102664

[5] N. Andersson and K. Glampedakis A superradiance resonance cavity outside rapidly rotating black holes Phys. Rev. Lett. 84 (2000), 4537-4540.

[6] S. Aretakis Decay of axisymmetric solutions of the wave equation on extreme Kerr backgrounds, Journal of Functional Analysis, 263 (2012), no. 9, 2770-2831.

[7] S. Aretakis Horizon Instabilities of Extremal Black Holes, to appear in ATMP, arXiv:1206.6598.

[8] S. Aretakis Stability and instability of extreme Reissner-Nordström black hole spacetimes for linear scalar perturbations I, Comm. Math. Phys. 307 (2011), no. 1, 17-63.

[9] S. Aretakis Stability and instability of extreme Reissner-Nordström black hole spacetimes for linear scalar perturbations II, Ann. Henri Poincaré 12 (2011), no. 8, 1491-1538.

[10] A. Bachelot and A. Motet-Bachelot Les résonances d'un trou noir de Schwarzschild Ann. Inst. H. Poincaré Phys. Théor. 59 (1993), no. 1, 368.

[11] P. Blue and A. Soffer Semilinear wave equations on the Schwarzschild manifold. I. Local decay estimates, Adv. Differential Equations 8 (2003), no. 5, 595-614.

[12] P. Blue and A. Soffer Errata for "Global existence and scattering for the nonlinear Schrodinger equation on Schwarzschild manifolds", "Semilinear wave equations on the Schwarzschild manifold I: Local Decay Estimates", and "The wave equation on the Schwarzschild metric II: Local Decay for the spin 2 Regge Wheeler equation", gr-qc/0608073, 6 pages.

[13] P. Blue and J. Sterbenz Uniform decay of local energy and the semi-linear wave equation on Schwarzschild space Comm. Math. Phys. 268 (2006), no. 2, 481-504.

[14] J. F. Bony and D. Häfner Decay and non-decay of the local energy for the wave equation in the de SitterSchwarzschild metric Comm. Math. Phys. 282 (2008), no. 3, 697-719.

[15] B. Carter Black hole equilibrium states, in Black Holes (Les Houches Lectures), edited B. S. DeWitt and C. DeWitt (Gordon and Breach, New York, 1972).

[16] B. Carter Hamilton-Jacobi and Schrödinger separable solutions of Einstein's equations Comm. Math. Phys. 10 (1968), 280-310.

[17] S. Chandrasekhar The mathematical theory of black holes, Oxford University Press, 1983.

[18] D. Civin Stability of subextremal Kerr-Newman exterior spacetimes for linear scalar perturbations, preprint, 2014

[19] D. Christodoulou The action principle and partial differential equations, Ann. Math. Studies No. 146, 1999

[20] D. Christodoulou and S. Klainerman The global nonlinear stability of the Minkowski space, Princeton University Press, 1993

[21] M. Dafermos The interior of charged black holes and the problem of uniqueness in general relativity Comm. Pure Appl. Math. 58 (2005), 0445-0504.

[22] M. Dafermos and I. Rodnianski A proof of Price's law for the collapse of a self-gravitating scalar field, Invent. Math. 162 (2005), 381-457.

[23] M. Dafermos and I. Rodnianski The redshift effect and radiation decay on black hole spacetimes Comm. Pure Appl. Math. 52 (2009), 859-919.

[24] M. Dafermos and I. Rodnianski The wave equation on Schwarzschild-de Sitter spacetimes, arXiv:0709.2766v1 [gr-qc]. 
[25] M. Dafermos and I. Rodnianski A note on energy currents and decay for the wave equation on a Schwarzschild background, arXiv:0710.0171v1 [math.AP].

[26] M. Dafermos and I. Rodnianski A proof of the uniform boundedness of solutions to the wave equation on slowly rotating Kerr backgrounds, Invent. Math. 185 (2011), no. 3, 467-559.

[27] M. Dafermos and I. Rodnianski Lectures on black holes and linear waves, Evolution equations, Clay Mathematics Proceedings, Vol. 17. Amer. Math. Soc., Providence, RI, 2013, pp. 97-205, arXiv:0811.0354 [gr-qc].

[28] M. Dafermos and I. Rodnianski A new physical-space approach to decay for the wave equation with applications to black hole spacetimes, in XVIth International Congress on Mathematical Physics, P. Exner (ed.), World Scientific, London, 2009, arXiv:0910.4957v1 [math.AP].

[29] M. Dafermos and I. Rodnianski The black hole stability problem for linear scalar perturbations, in Proceedings of the Twelfth Marcel Grossmann Meeting on General Relativity, T. Damour et al (ed.), World Scientific, Singapore, 2011, pp. 132189, arXiv:1010.5137

[30] M. Dafermos and I. Rodnianski Decay for solutions of the wave equation on Kerr exterior spacetimes I-II: The cases $|a| \ll M$ or axisymmetry, arXiv:1010.5132

[31] M. Dafermos, G. Holzegel and I. Rodnianski A scattering theory construction of dynamical black hole spacetimes, arXiv:1306.5534

[32] S. Dyatlov Quasi-normal modes and exponential energy decay for the for the Kerr-de Sitter black hole Comm. Math. Physics 306 (2011), 119-163.

[33] S. Dyatlov Exponential energy decay for Kerrde Sitter black holes beyond event horizons, Mathematical Research Letters 18 (2011), 1023-1035

[34] S. Dyatlov Asymptotics of linear waves and resonances with applications to black holes, arXiv:1305.1723

[35] F. Finster, N. Kamran, J. Smoller, S-T. Yau Decay of solutions of the wave equation in Kerr geometry Comm. Math. Phys. 264 (2006), 465-503.

[36] F. Finster, N. Kamran, J. Smoller, S-T. Yau Erratum: Decay of solutions of the wave equation in Kerr geometry Comm. Math. Phys., online first.

[37] A. Franzen, The wave equation on black hole interiors, Ph.D. Thesis, 2014

[38] O. Gannot Quasinormal modes for Schwarzschild-AdS black holes: exponential convergence to the real axis, arXiv:1212:1907

[39] S. W. Hawking and G. F. R. Ellis The large scale structure of space-time Cambridge Monographs on Mathematical Physics, No. 1. Cambridge University Press, London-New York, 1973.

[40] G. Holzegel, Ultimately Schwarzschildean spacetimes and the black hole stability problem, arXiv:1010.3216.

[41] G. Holzegel and J. Smulevici Decay properties of Klein-Gordon fields on Kerr-AdS spacetimes Comm. Pure Appl. Math. 66 (2013), no. 11, 1751-1802

[42] G. Holzegel and J. Smulevici Quasimodes and a Lower Bound on the Uniform Energy Decay Rate for Kerr-AdS Spacetimes, arXiv: 1303.5944

[43] B. Kay and R. Wald Linear stability of Schwarzschild under perturbations which are nonvanishing on the bifurcation 2-sphere Classical Quantum Gravity 4 (1987), no. 4, 893-898.

[44] S. Klainerman Uniform decay estimates and the Lorentz invariance of the classical wave equation Comm. Pure Appl. Math. 38 (1985), 321-332

[45] I. Laba and A. Soffer Global existence and scattering for the nonlinear Schrödinger equation on Schwarzschild manifolds Helv. Phys. Acta 72 (1999), no. 4, 272-294.

[46] P. Laul, J. Metcalfe, S. Tikare and M. Tohaneanu Localized energy estimates on Myers-Perry space-times, arXiv:1401.0465

[47] J. Lucietti and H. S. Reall Gravitational instability of an extreme Kerr black hole Phys. Rev. D 86 (2012), 104030

[48] J. Luk Improved decay for solutions to the linear wave equation on a Schwarzschild black hole, Ann. Henri Poincaré 11 (2010), no. 5, 805-880.

[49] J. Luk A Vector Field Method Approach to Improved Decay for Solutions to the Wave Equation on a Slowly Rotating Kerr Black Hole, Anal. PDE 5 (2012), no. 3, 553-625.

[50] J. Luk The null condition and global existence for nonlinear wave equations on slowly rotating Kerr spacetimes, JEMS 15 (2013), no. 5, 1629-1700.

[51] R. Melrose, A. Sá Barreto, A. Vasy Asymptotics of solutions of the wave equation on de Sitter-Schwarzschild space, arXiv:0811.2229.

[52] C. S. Morawetz Time decay for the nonlinear Klein-Gordon equations Proc. Roy. Soc. Ser. A 206 (1968), $291-296$.

[53] G. Moschidis, forthcoming.

[54] K. Murata, H. S. Reall and N. Tanahashi What happens at the horizon(s) of an extreme black hole? Class. Quantum Grav. 30 (2013) 235007

[55] J. Ralston Solutions of the wave equation with localized energy Comm. Pure Appl. Math. 22 (1969), 807-823.

[56] J. Sbierski Characterisation of the Energy of Gaussian Beams on Lorentzian Manifolds - with Applications to Black Hole Spacetimes, arXiv:1311.2477

[57] V. Schlue Decay of linear waves on higher dimensional Schwarzschild black holes, Analysis \& PDE 6, (2013), $515-600$ 
[58] V. Schlue Global results for linear waves on expanding Kerr and Schwarzschild de Sitter cosmologies, arXiv: $1207.6055 \mathrm{v} 2$

[59] Y. Shlapentokh-Rothman Quantitative Mode Stability for the Wave Equation on the Kerr Spacetime, arXiv:1302.6902, to appear in Ann. Henri Poincaré.

[60] Y. Shlapentokh-Rothman Exponentially growing finite energy solutions for the Klein-Gordon equation on subextremal Kerr spacetimes, arXiv:1302.3448, to appear in Commun. Math. Phys.

[61] A. Starobinsky Amplification of waves during reflection from a black hole Soviet Physics JETP 37 (1973), $28-32$.

[62] D. Tataru Local decay of waves on asymptotically flat stationary space-times, Amer. J. Math. 135 (2013), no. 2, 361-401.

[63] D. Tataru and M. Tohaneanu Local energy estimate on Kerr black hole backgrounds, IMRN (2011), no. 2, 248292.

[64] A. Vasy Microlocal analysis of asymptotically hyperbolic and Kerr-de Sitter spaces, with an appendix by Semyon Dyatlov Invent. Math. 194 (2013), 381-513.

[65] B. Whiting Mode stability of the Kerr black hole J. Math. Phys. 30 (1989), 1301.

[66] J. Wunsch and M. Zworski Resolvent estimates for normally hyperbolic trapped sets Ann. Inst. Henri Poincaré (A), 12 (2011), 1349-1385.

[67] S. Yang Global solutions to nonlinear wave equations in time dependent inhomogeneous media, Arch. Ration. Mech. Anal. 209 (2013), no. 2, 683-728.

[68] S. Yang Global stability of solutions to nonlinear wave equations, arXiv:1205.4216.

[69] S. Yang On the quasilinear wave equations in time dependent inhomogeneous media, arXiv:1312.7246.

Princeton University, Department of Mathematics, Fine Hall, Washington Road, Princeton, NJ 08544, United STATES, dafermos@math.princeton.edu

University of Cambridge, Department of Pure Mathematics and Mathematical Statistics, Wilberforce Road, Cambridge CB3 0WA, United Kingdom, M.Dafermos@dpmms.cam.ac.uk

Princeton University, Department of Mathematics, Fine Hall, Washington Road, Princeton, NJ 08544, UNITED STATES, irod@math.princeton.edu

Massachusetts Institute of Technology, Department of Mathematics, 77 Massachusetts Avenue, CamBRIDGE, MA 02139, UNITED STATES, irod@math.mit.edu

Massachusetts Institute of Technology, Department of Mathematics, 77 Massachusetts Avenue, CamBRIDGE, MA 02139, United StATES, yakovsr@math.mit.edu 